\title{
Social background, educational attainment and labor market integration: an exploration of underlying processes and dynamics
}

Citation for published version (APA):

Büchner, C. I. R. (2013). Social background, educational attainment and labor market integration: an exploration of underlying processes and dynamics. [Doctoral Thesis, Maastricht University]. ROA. https://doi.org/10.26481/dis.20131211cb

Document status and date:

Published: 01/01/2013

DOI:

10.26481/dis.20131211cb

Document Version:

Publisher's PDF, also known as Version of record

Please check the document version of this publication:

- A submitted manuscript is the version of the article upon submission and before peer-review. There can be important differences between the submitted version and the official published version of record.

People interested in the research are advised to contact the author for the final version of the publication, or visit the DOI to the publisher's website.

- The final author version and the galley proof are versions of the publication after peer review.

- The final published version features the final layout of the paper including the volume, issue and page numbers.

Link to publication

\footnotetext{
General rights rights.

- You may freely distribute the URL identifying the publication in the public portal. please follow below link for the End User Agreement:

www.umlib.nl/taverne-license

Take down policy

If you believe that this document breaches copyright please contact us at:

repository@maastrichtuniversity.nl

providing details and we will investigate your claim.
}

Copyright and moral rights for the publications made accessible in the public portal are retained by the authors and/or other copyright owners and it is a condition of accessing publications that users recognise and abide by the legal requirements associated with these

- Users may download and print one copy of any publication from the public portal for the purpose of private study or research.

- You may not further distribute the material or use it for any profit-making activity or commercial gain

If the publication is distributed under the terms of Article $25 \mathrm{fa}$ of the Dutch Copyright Act, indicated by the "Taverne" license above, 
Social Background, Educational Attainment and Labor Market Integration:

An Exploration of Underlying

Processes and Dynamics 
(C) Charlotte Ilse Ruth Büchner, Maastricht 2013

All rights reserved. No part of this publication may be reproduced, stored in a retrieval system, or transmitted in any form, or by any means, electronic, mechanical, photocopying, recording, or otherwise, without the prior permission in writing, from the author.

Published by ROA

Postbus 616

6200 MD Maastricht

ISBN: 978-90-5321-518-0

Printed in the Netherlands by Océ Business Services 


\title{
Social Background, Educational Attainment and Labor Market Integration: \\ An Exploration of Underlying Processes and Dynamics
}

\author{
Proefschrift \\ ter verkrijging van de graad van doctor aan \\ de Universiteit Maastricht, \\ op gezag van de Rector Magnificus, \\ Prof. dr. L.L.G. Soete \\ volgens het besluit van het College van Decanen, \\ in het openbaar te verdedigen op \\ woensdag 11 december 2013 om 16.00 uur \\ door \\ Charlotte Ilse Ruth Büchner
}




\section{Promotor}

Prof. dr. R. K. W. van der Velden

\section{Copromotor}

Dr. M. H. J. Wolbers

\section{Thesis committee}

Prof. dr. L. Borghans (voorzitter)

Prof. dr. J. Dronkers

Prof. dr. M. P. C. van der Werf (University of Groningen)

Prof. dr. H. G. van de Werfhorst (University of Amsterdam) 


\section{Acknowledgements}

I am deeply grateful to my promotor Rolf van der Velden and co-promotor Maarten Wolbers for guiding and supporting me throughout the years when I worked on this thesis. Without their valuable suggestions, knowledge and advice, this dissertation would not have been possible. Thank you, Rolf, also for supporting me with your enthusiasm, your patience and motivation to complete this thesis. Thank you, Maarten, for our fruitful meetings and discussions about specific research issues, your useful feedback and critical comments on the papers and the final manuscript.

An essential part of this thesis builds the use of large and unique data sets coming from Statistics Netherlands. I thank all colleagues from Statistics Netherlands who provided me with these data and their help and assistance. Special thanks go to Tanja Traag and Wendy Smits for their invaluable support and their cooperation as my co-authors. I also would like to thank the Netherlands Organisation for Scientific Research (NWO) for funding this $\mathrm{PhD}$ project and the members of the assessment committee for their time and willingness to evaluate this thesis.

There are many people who provided useful comments and inspiring discussions on different parts of the dissertation. For comments on earlier versions of the chapters of this study I am especially indebted to Eric Bonsang, Bart Golsteyn, Martin Humburg, Ben Kriechel, Mark Levels, Olivier Marie, Arjan Non, Erik Plug and Jan Sauermann. This thesis also benefited from helpful feedback of the seminar and conference participants from EQUALSOC, ESPE, ISA-RC28, and the SOFI in Stockholm.

I am grateful to the Research Centre for Education and the Labour Market (ROA) at Maastricht University and to my colleagues there who provided a great and pleasant work environment, in which I could learn and grow. Special thanks go to the ROA-secretariat for 
their constantly kind support in administrative matters, to Joyce Gruijthuijsen for her great help and assistance throughout the years, and to Mariëlle Retz for her support, especially during the last months and weeks of finalizing this thesis.

Last but not least, writing this thesis would also not have been possible without the love and support of my friends and entire family. I wish to thank my friends in Germany and Maastricht for the good times we had also outside the university world and that I always could count on you. I particularly thank Anna, Elena and Myrthe for always being great friends. Also thanks to Anant, Antonio, Laura, Martin, Petr, Eric, Jan, and Olivier for making Maastricht an unforgettable and special place to me.

My deep love and gratitude go to my parents, who never lost faith in me and provided their unconditional love and support in all circumstances. Finally, I am grateful to my daughter Ava, who was born in the last period of my PhD-writing and who was always my dearly beloved distraction and drive.

Berlin, Germany

Charlotte I. R. Büchner

November, 2013 


\section{Content}

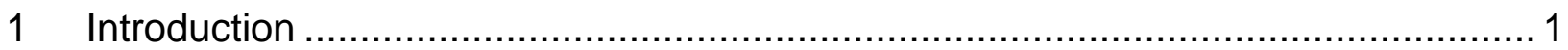

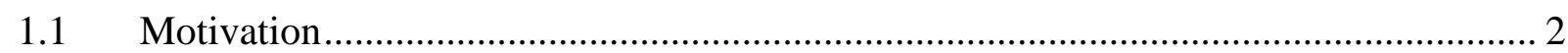

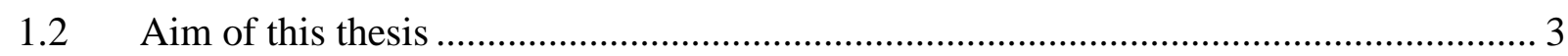

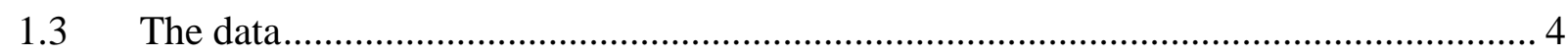

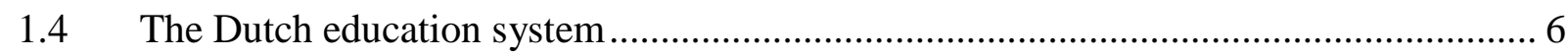

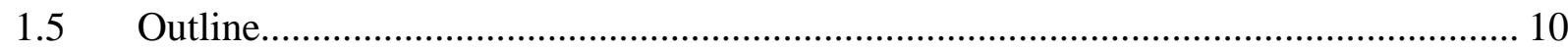

2 How social background affects educational attainment over time in the

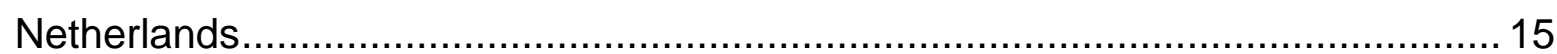

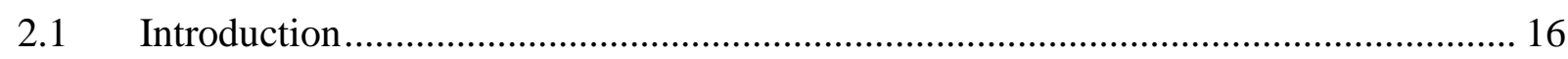

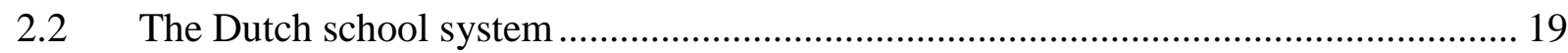

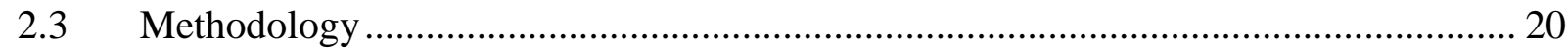

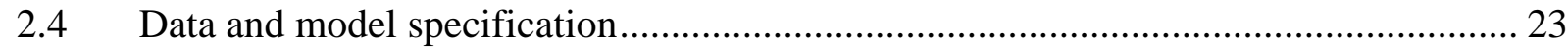

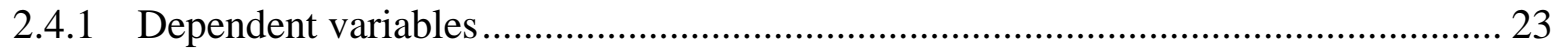

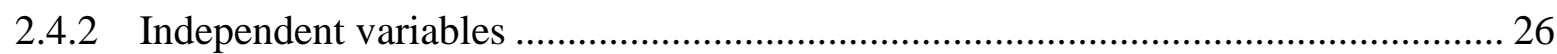

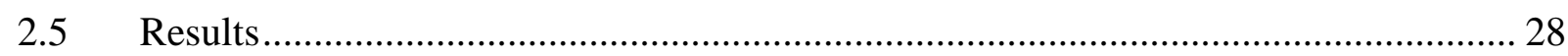

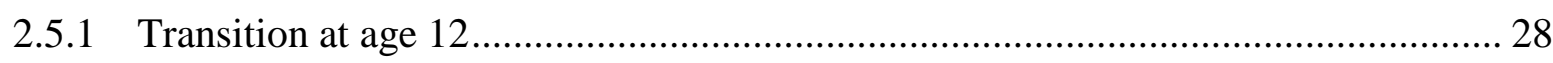

2.5.2 Primary and secondary effects at the age 12 transition......................................... 29

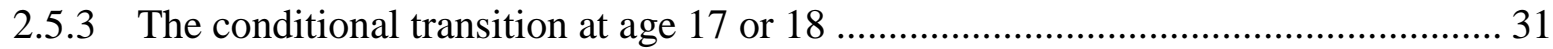

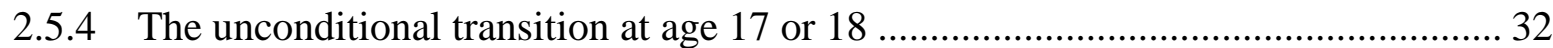

2.5.5 Primary and secondary effects at the age $17-18$ transition...................................... 34

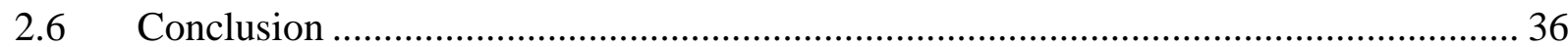




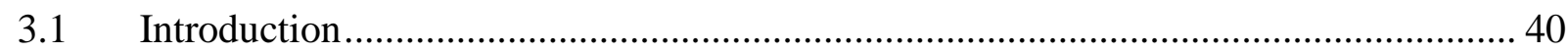

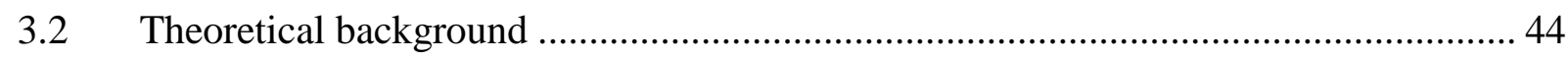

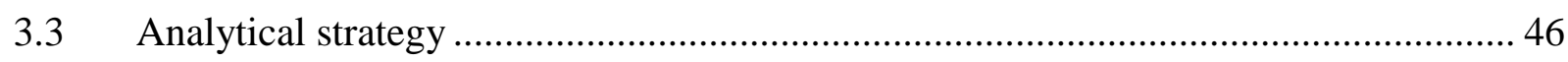

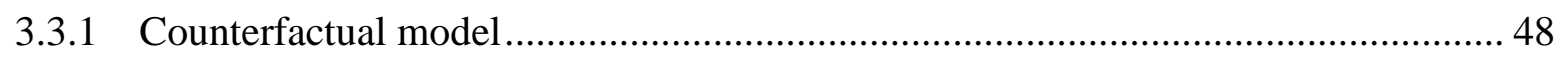

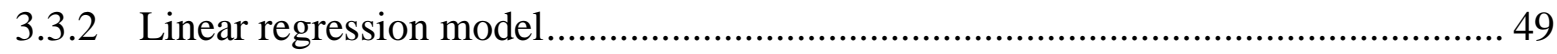

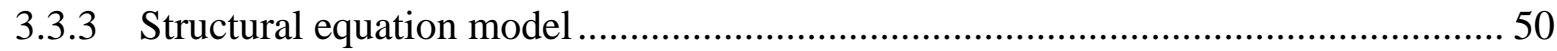

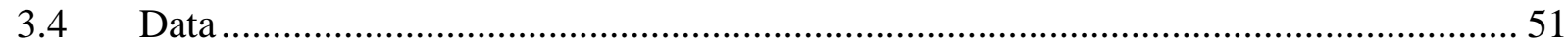

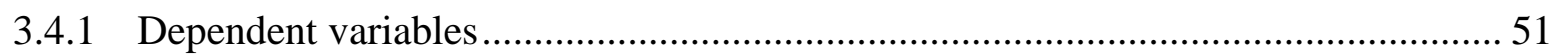

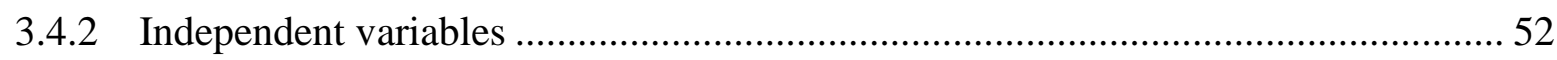

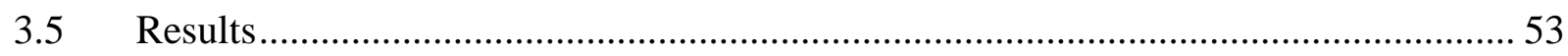

3.5.1 Primary and secondary effects’ relative importance using counterfactual models... 55

3.5.2 Primary and secondary effects’ relative importance using linear regression models

3.5.3 Primary and secondary effects' relative importance using structural equation

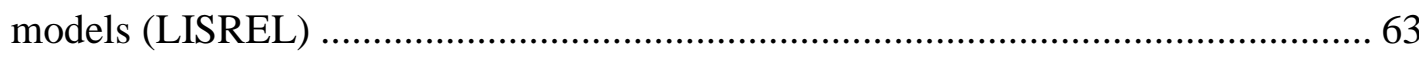

3.5.4 Comparing the findings from the three statistical methods .................................. 68

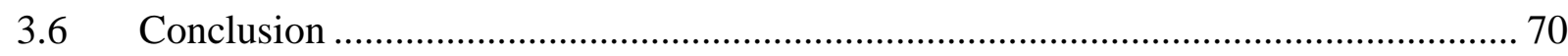

4 Education, cognitive skills, and earnings of males and females...................... 73

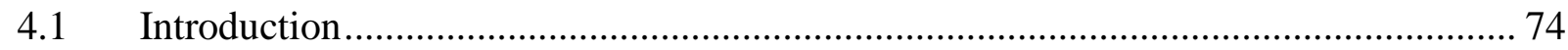

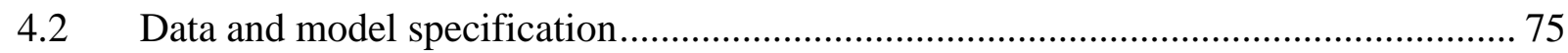

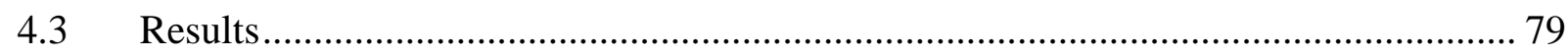

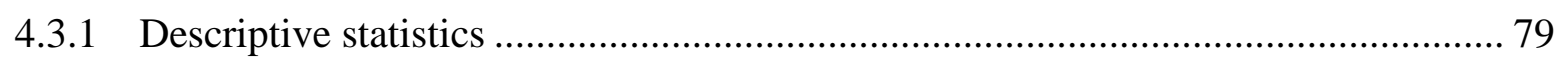

4.3.2 Estimation results for males' and females' educational outcomes ........................... 82

4.3.3 Estimation results for males’ and females’ labor market outcomes ........................ 85

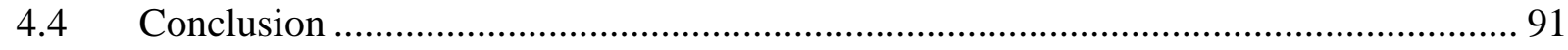

5 How education, cognitive skills, and cultural and social capital account for intergenerational earnings persistence ............................................. 93

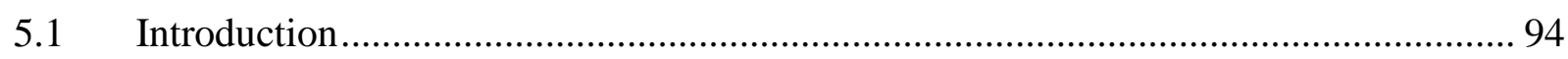

$5.2 \quad$ Modeling approach and estimation procedure ........................................................ 96

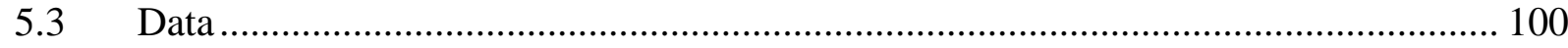

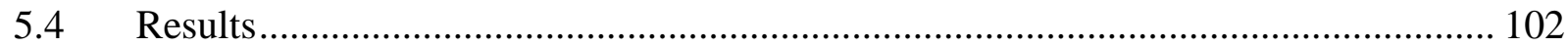




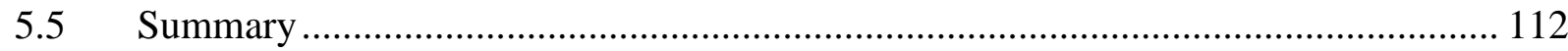

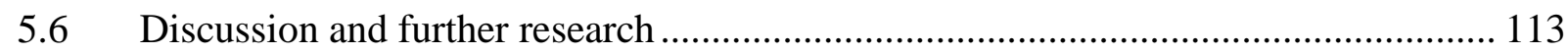

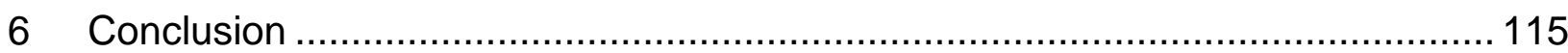

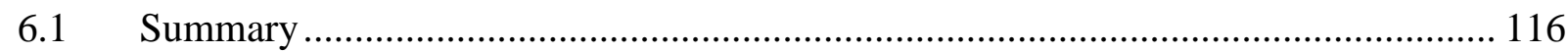

6.1.1 How social background affects educational attainment over time in the

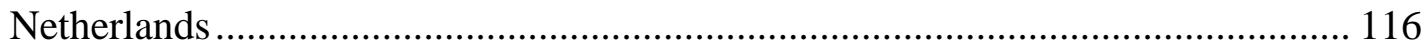

6.1.2 Social background's effect on educational attainment: Does method matter? ....... 118

6.1.3 Education, cognitive skills and earnings of males and females.............................. 120

6.1.4 How education, cognitive skills, cultural and social capital account for intergenerational earnings persistence................................................................... 121

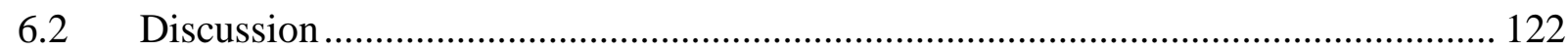

6.2.1 Social inequality and the role of education........................................................ 122

6.2.2 Gender and the role of education ....................................................................... 125

6.2.3 Cognitive skills measurement and individual outcome ………………………….... 126

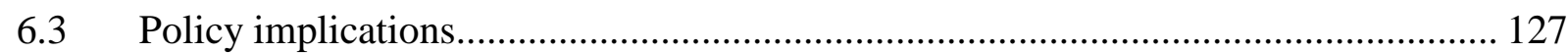

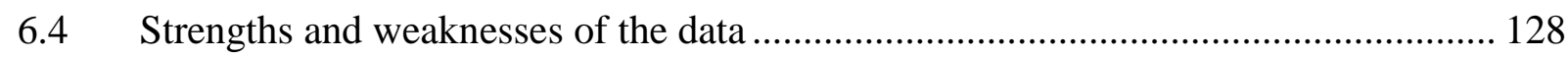

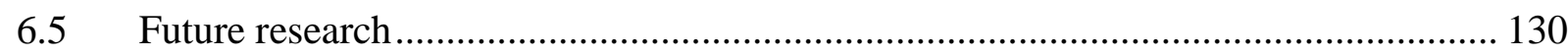

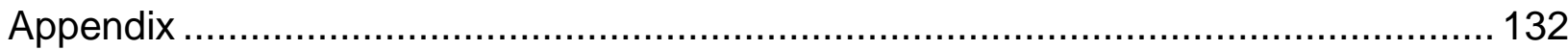

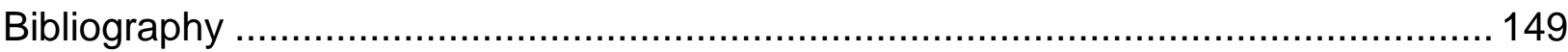

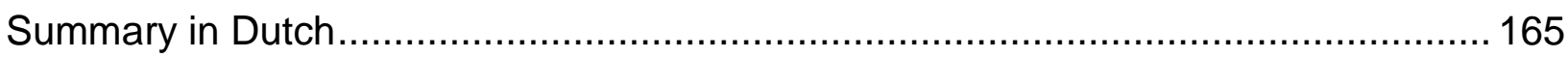

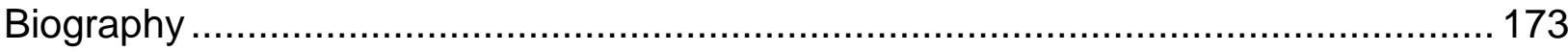

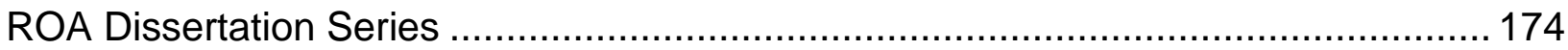




\section{List of Figures}

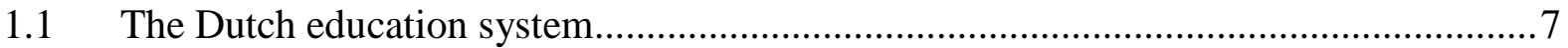

1.2 Share of pupils with a final diploma in secondary education tracks, in the relevant age-range, for the school years 1974 to 2007 (3-year moving averages) ....................... 9

1.3 Share of pupils with a final diploma in postsecondary education tracks, in the relevant age-range, for the years 1974 to 2007 (3-year moving averages) ...................10

2.1 Transition and completion rates in secondary and postsecondary education, 1993 school cohort.....

3.1 Academic performance and transition probabilities to academic tracks in secondary education, school cohort 1993

3.2 Academic performance and transition to academic tracks in postsecondary education, school cohort 1993.

3.3 Structural equation model (with latent variable cognitive skills) on transition to

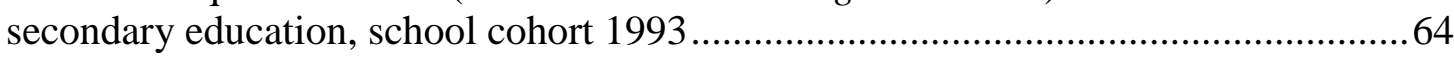

3.4 Structural equation model (with latent variable cognitive skills) on final educational degree attained, school cohort 1993.

3.5 Structural equation model (with latent variable cognitive skills and social background) on transition to secondary education, school cohort 1993 .66

3.6 Structural equation model (with latent variable cognitive skills and social background) on final educational degree attained, school cohort 1993.

4.1 Males' probability of being graduated from college or university at cognitive skills..83

4.2 Females' probability of being graduated from college or university at cognitive skills

4.3 Probability of graduating from engineering studies at math scores, higher educated males and females

4.4a Log earnings at math scores, controlled for math squared, males and females ...........89

4.4b Log earnings at language scores, controlled for language squared, males and females

4.4c Log earnings at IQ scores, controlled for IQ squared, males and females ..................90

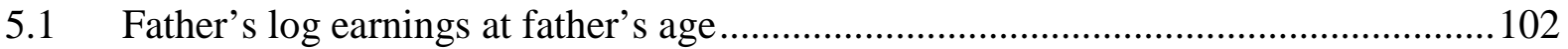

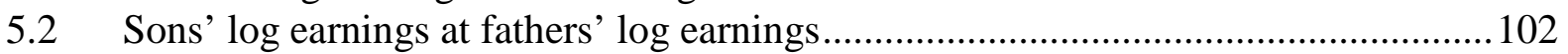




\section{List of Tables}

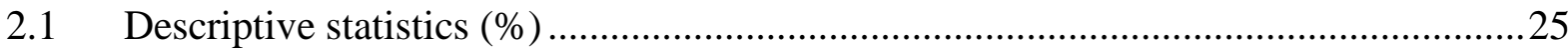

2.2 Means and standard deviations of standardized performance scores at age 12,

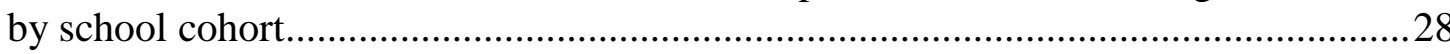

2.3 Estimated factual and counterfactual transition rates (\%), from primary education

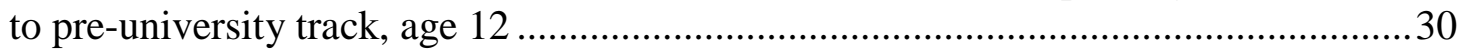

2.4 Relative importance (\%) of secondary effects at age-12 transition from primary education to pre-university track and precollege track or pre-university track............30

2.5 Estimated factual and counterfactual transition rates (\%), from pre-university track to university, conditional transition at age 17 or 18 ....................................................33

2.6 Estimated factual and counterfactual transition rates (\%) of all students to university, unconditional transition at age 17 or 18

2.7 Relative importance (\%) of secondary effects at conditional transition at age 17 or 18: from pre-university track to university and from precollege track or preuniversity track to vocational college or university...

2.8 Relative importance (\%) of secondary effects at unconditional transition at age 17 or 18: all students to university, and to vocational college or university

3.1a Estimated probabilities of the transition to higher secondary education

for actual and counterfactual transition rates

3.1b Estimated probabilities of completing academic tracks in secondary education for actual and counterfactual transition rates.....

3.1c Estimated probabilities of the transition to higher postsecondary education for actual and counterfactual transition rates.

3.1d Estimated probabilities of completing academic tracks in postsecondary education for actual and counterfactual transition rates.............................................................5

3.1e Estimated probabilities of final academic tracks in postsecondary education: unconditional models for actual and counterfactual transition rates

3.2a Relative importance of secondary effects, at the transition to academic tracks in secondary and postsecondary education (log odds ratios).

3.2b Relative importance of secondary effects, at the completion of academic tracks in secondary and postsecondary education (log odds ratios) ......................................58

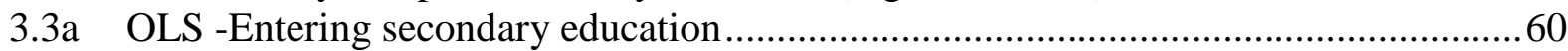

3.3b OLS - Completing secondary education given secondary education attended ............60

3.3c OLS - Attending postsecondary education given secondary education completed.....60 
3.3d OLS - Completing postsecondary education given postsecondary education attended.

3.3e OLS - Final education (unconditional model), for those who attained a certificate in higher postsecondary education

3.4 OLS: Relative importance of primary and cumulative secondary effects on educational transitions and completions

3.5 Summary of the relative importance of direct and cumulative secondary effects, obtained by different statistical methods, in \%

4.1 Summary statistics of the selected sample

4.2 Summary statistics of selected variables, males and females specified by educational level

4.3 OLS on years of schooling, separate for males and females

4.4 Multinomial logit regression on field of study choice of males and females in higher education (predicted marginal effects)

4.5 Estimated coefficients from log earning regressions, specified by educational level, males.

4.6 Estimated coefficients from log earning regressions, specified by educational level, females

5.1 Income elasticities 104

5.2 Relationships between transition mechanisms, father's log earnings and son's log earnings.....

5.3 Accounting for the relationship between father's earnings and son's earnings .........108

5.4 Relationships between transition mechanisms, father's log earnings and son's log house property value (selected sample).

5.5 Accounting for the relationship between father's log earnings and son's log house property value 
1 Introduction 


\subsection{Motivation}

Education is a key factor that contributes to a child's personal and social development and also positively affects other dimensions in an individual's life course (Organisation for Economic Co-operation and Development (OECD), 2011). In the Netherlands, like in many other Western European countries, the educational level attained is a component used by employers to screen people at the labor market and to predict labor market productivity. A higher educational degree is therefore associated with higher labor participation and higher earnings. In turn, a lack of (formal) education is connected to social and financial risks. The underlying process of educational attainment and labor market integration, however, is still not fully understood. There are several underlying mechanisms, such as the family background, cognitive skills and performance, and their interplay with the structural conditions of the education system. These mechanisms are likely to exert a considerable impact on an individual's educational and labor market success and to be jointly responsible for the level of inequality across social groups (Jackson, 2013). For example, the family environment is the first place of a child's educational experiences. It builds the basis for the linguistic, cognitive and social development and is decisive in stimulating and boosting a child's talents and educational performance, but also influences the offspring's educational choices. The family background thus contributes indirectly and directly to the educational outcome, as both performance and (subsequent) educational choices are relevant predictors of the final educational level attained. As performance and educational choices are likely to differ across social groups, the direct and indirect effects of family background also have a significant share in determining the level of inequality of educational opportunity (IEO). The relative size of these direct and indirect effects, however, can alter over a child's educational career and over time, and thus also alters the size of IEO (Boudon, 1974, Jackson and Jonsson, 2013).

This has important implications for social policies: Strategies aiming to reduce the social gap in educational opportunities can be more precise, if these processes are disentangled and their varying, relative impact in this relationship is identified. However, it is not always easy to clearly decompose these processes and to detect their relative contributions to IEO throughout the educational career and over time, or to investigate the subsequent consequences these mechanisms have for labor market outcomes. In this regard, education is often considered a 'black box', which is also related to a lack of adequate data, especially representative longitudinal data. 


\subsection{Aim of this thesis}

This thesis aims to open parts of the 'black box', by using unique data sets of panel and administrative data information and to shed some light on the mechanisms contributing to educational attainment and the development and size of IEO, as well as on the mechanisms underlying the association between education and labor market outcomes in the Netherlands. The central issues addressed are:

- What is the relative role of direct and indirect effects of social background in explaining educational choices and attainment, and thus the size of IEO?

- What can be said about the underlying mechanisms associated with educational attainment and their relationship with labor market outcomes?

- What is the relative importance of transmission mechanisms that account for the association between a father's and his son's earnings?

Attention thereby is paid, among others, to the structural features of the Dutch education system, as they build an integral part in the above-mentioned relations. In the Netherlands, parents make important educational decisions for their child - under the consideration of teachers' recommendations and performance test results in primary schools - and determine the educational pathways, especially in the beginning of the educational career. Thus, families and schools are reciprocally interacting in their influence on a child's educational career. Socially privileged families thereby may be - more than less advantaged families strategic in using the possibilities the education system provides to induce a head start for their children. Social differences in behavior and institutional 'loopholes' in the education system, however, make educational selection and transition processes vulnerable to IEO (Jackson and Jonsson 2013). Policy actions that aim to reduce IEO thus are likely to fail, if these circumstances are not considered. ${ }^{1}$

Against the background of IEO it is a first aim of the thesis to investigate the extent to which social background accounts for differences in educational outcomes in the Netherlands and whether or not this has changed over time. We will answer this question by using the theoretical approach of Boudon (1974) on primary and secondary effects of social stratification and testing the implications empirically, by applying the estimation method of Erikson, Goldthorpe, Jackson, Yaish, and Cox (2005) and Jackson, Erikson, Goldthorpe, and

\footnotetext{
${ }^{1}$ Related, Blossfeld and Shavit (1993) point out that educational expansion as a tool to create more equality of educational opportunities may have increased the average level of educational attainment in a society, but did not necessarily change the relative proportions between children from different social backgrounds in higher education. For a critical discussion about the education system, see also Chapter 6, section 6.2.1.
} 


\section{Introduction}

Yaish (2007). Using large datasets that consist of representative cohort samples and administrative data, we contribute to the existing literature by having a detailed view on educational pathways, performance and background-related transition behavior and comparisons over a longer period. In addition, we are able to evaluate our results in the context of a cross-national comparison (cf. Jackson and Jonsson, 2013). In a next step, we apply different estimation techniques to test for the robustness of our findings and to shed some light on previous results in this field. From our findings we will draw policy relevant conclusions about the openness of the Dutch educational system and the institutional support of equal chances.

Extending the perspective on labor market outcomes, it is a second aim of this thesis to explore the role of education and cognitive skills in explaining differences in labor market outcomes both for males and females in the Netherlands. Including the gender perspective enhances the view on horizontal differences beyond the social stratification debate. Moreover, we measure cognitive skills by several tests which provide a better understanding about the predictive power of different cognitive skills proxies. ${ }^{2}$

A third aim of the thesis is to examine transmission mechanisms that account for the intergenerational earnings persistence between fathers and sons. Estimating intergenerational income mobility as the association between a father's and his son's earnings is a classical approach in the economic literature. However, this strand of literature largely neglects the transmission mechanisms accounting for this association. The sociological literature, in contrast, considers transmission mechanisms extensively, but rather in the context of status mobility than earnings mobility. By including the son's education and cognitive skills, as well as the parents' cultural capital and the social capital of the family's neighborhood as transmission mechanisms in this relationship, we follow a relatively new approach that combines both strands of literature and adds another, interdisciplinary aspect to the research on social mobility and inequality.

\subsection{The data}

A clear advantage of this thesis is the use of large unique data sets, provided by Statistics Netherlands (CBS). This section gives a general overview of the data that have been used

\footnotetext{
2 See also Almlund, Duckworth, and Heckman (2011) and Borghans, Golsteyn, Heckman, and Humphries (2011).
} 


\section{Introduction}

throughout this thesis. A detailed description of the (matched) data sets for specific analyses can be found in the data section of each chapter.

The basis of the analyses in all chapters are one or more education cohorts; the socalled Secondary Education Student Cohorts. Each of them is a large-scale, nationally representative longitudinal survey for the Netherlands. Data are collected via interviews with the students, their parents ${ }^{3}$ and teachers and via administrative information by the schools who participate in the survey. The students in each cohort are followed from age 12 onwards, when they enter secondary education, until the end of their full-time education or until age 28.

The first cohort starts collecting information in $1977(N=37,280)$, the second in 1982 ( $N=16,813)$, the third in $1989(N=19,524)$, and the fourth in $1993(N=20,331)$. Each dataset provides comparable information on the socioeconomic background and standardized skills tests of pupils at the entry to secondary education. The socioeconomic background information comprises, as a minimum, data about parents' educational and occupational level, their cultural activities, and their support in school-related issues of the child (e.g. discussions about school performance). The skills tests include a math and a language test as well as a test on non-verbal intelligence. The math and language tests are based on the socalled CITO test, a nationwide standardized school performance test. Each test comprises 20 multiple choice questions. The non-verbal intelligence test is on logical reasoning and the capacity to abstract (Horn, 1969), and comprises 40 items.

School track choices and whether a final examination was passed are provided each year by the schools for all pupils. We measure the highest educational level attained in years, constructed on a scale ranging from 6 years, being the lowest level, to 19 years, being the highest level, which is completing university. ${ }^{4}$

For the analysis on labor market outcomes, the survey data are enriched with administrative data coming from municipalities and national tax registers. They provide, among others, information on labor force status and earnings of the respondents and their partners, as well as the family composition of the respondent's household (for instance, marital status, number and age of children). The fact that these data are from registers guarantees a high quality, especially of the earnings data.

\footnotetext{
${ }^{3}$ Parental information on social status and family characteristics are surveyed once, at the start of each cohort sample.

${ }^{4}$ This is according to the 'years of schooling ladder' by Bosker, Hofman and Van der Velden (1985).
} 


\subsection{The Dutch education system}

As mentioned before, the data enable us to follow students throughout their educational career. The structural features of the Dutch education system thereby play a crucial role in how students proceed in their educational career and to what extent equality in educational opportunities is provided. This section gives an overview of the Dutch educational system since the introduction of the Mammoth Law in 1968, and describes the system's main characteristics.

The education system in the Netherlands is highly stratified from secondary education onward and can generally be divided into vocationally oriented tracks and academic tracks. The first transition, from primary education to secondary education, takes place at age 12 . The transition to upper secondary vocational education and apprenticeship training takes place at age 16, and to higher education (vocational colleges and universities) takes place at age 17 or 18, depending on which secondary education track the student followed (see Figure 1.1). The structure of the secondary education system in the Netherlands was largely shaped by the Mammoth Law of 1968, which moved the country toward a more integrated system. Before this law came into effect, secondary education consisted of four educational tracks organized in different institutions. Pupils were admitted to one of these tracks at age 12 on the basis of entry examinations, and they had little or no possibility of moving to a higher track. The Mammoth Law maintained the different hierarchical school types of the old system but facilitated transitions to secondary education through orientation classes (brugklas) in the first and sometimes second grade of secondary education. These orientation classes were meant to postpone track placement until the end of the first or second grade. In many cases the orientation classes combine two adjacent tracks, but schools are autonomous in the way that they implement this: some maintain separate classes for each track, and others combine all four tracks of secondary education. The Mammoth Law also stimulated the formation of school communities in which different tracks of secondary education were combined within one building, which made it possible for more students to change tracks without changing schools. Furthermore, at the end of primary school, nationwide standardized skill tests (the so-called CITO $^{5}$ tests) and class teacher recommendations were introduced to structure the selection into different tracks in secondary education (see Dronkers, 1993:262-63). The CITO tests are used to measure school performance in the final

\footnotetext{
${ }^{5}$ CITO stands for Centraal Instituut voor Toets Ontwikkeling, which is the Dutch central institute for test development that provides the tests to primary schools nationwide.
} 


\section{Introduction}

grade of primary education in language, mathematics, and information processing. Although the CITO tests are not mandatory, most primary schools (about 92\%) use them to assess their pupils (CITO, 2010).

Figure 1.1: The Dutch education system

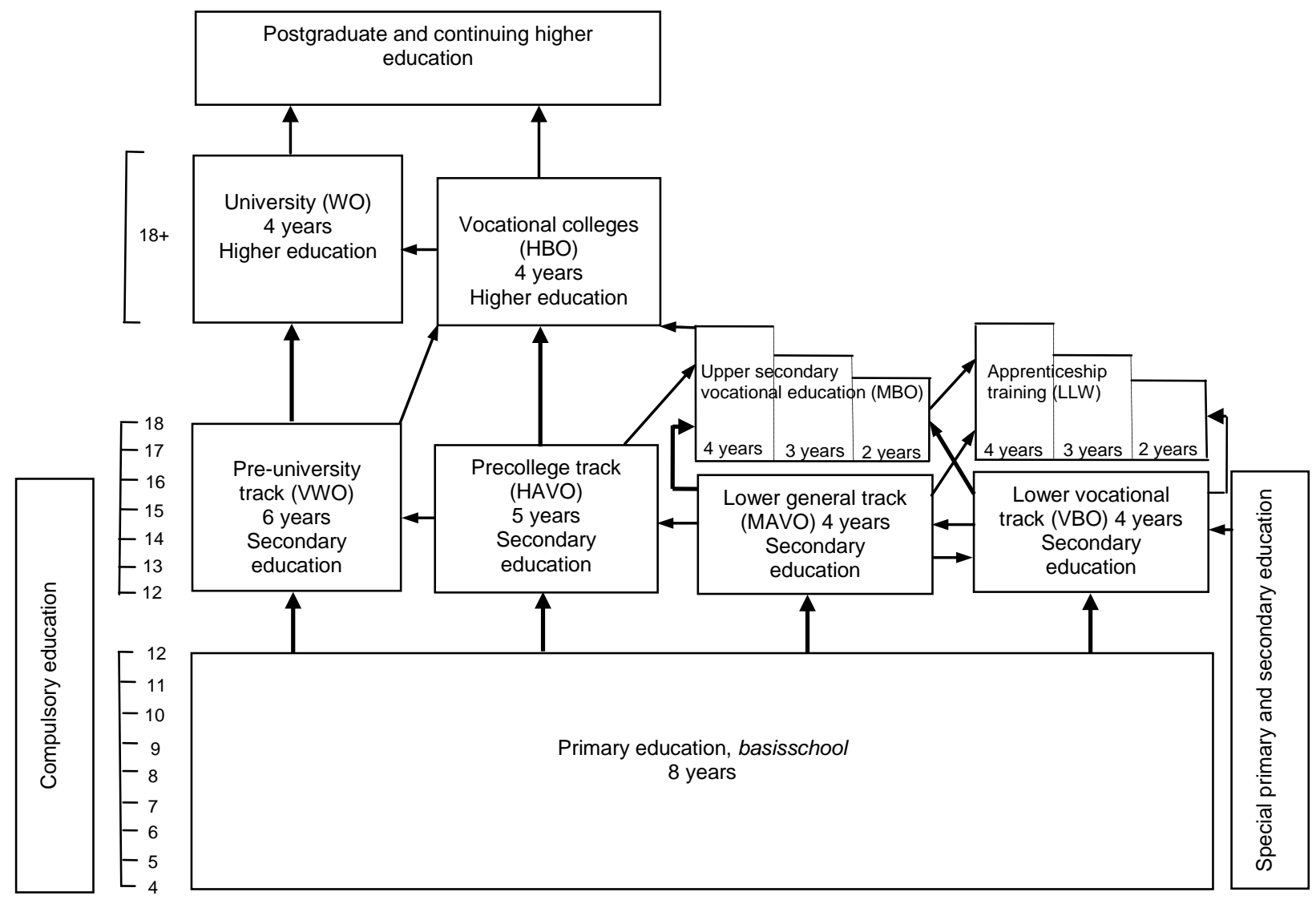

As shown in Figure 1.1, compulsory education starts with primary education at age 4 and ends at age $16 .{ }^{6}$ Primary education, organized in the so-called basisschool, lasts for eight years, until age 12. Secondary education is distinguished in four tracks, three general and one vocational:

- pre-university education (VWO): the pre-university track

- higher general secondary education (HAVO): the precollege track

- intermediate general secondary education (MAVO): the lower general track

- prevocational education (VBO): the lower vocational track

Both primary education and secondary education also have a system of special education for pupils with learning problems or physical disabilities.

\footnotetext{
${ }^{6}$ At age 17 students are still obliged to follow formal education for at least two days per week.
} 
The arrows in Figure 1.1 indicate the possible routes for proceeding from one track to another in the education system, with bold arrows indicating the main routes. Vertical upward transitions to the higher school tracks are allowed when appropriate levels beneath them have been completed. Downward transitions to lower tracks are possible at any time. Only the two highest tracks in secondary education prepare students for higher education: the precollege track (HAVO) prepares for vocational colleges (HBO) and the pre-university track (VWO) prepares for university (WO). Both the lower general track (MAVO) and the lower vocational track (VBO) mainly prepare for upper secondary vocational education (MBO). In general, the lower general track (MAVO) aims to prepare students for the long (three- or four-year) tracks in upper secondary vocational education (MBO), while the lower vocational track (VBO) aims to prepare for the short (one- or two-year) tracks and apprenticeship training. Each track after primary education follows a specified path to the next track. The system thereby also allows transitions within the secondary and postsecondary levels. The most relevant within-level transitions at the secondary level are from the lower general track (MAVO) to the precollege track (HAVO) and from the precollege track (HAVO) to the preuniversity track (VWO). At the postsecondary level, the most relevant transitions are from upper secondary vocational education (MBO) to vocational colleges (HBO) and from vocational colleges (HBO) to university (WO).

In 2000 the Dutch Ministry of Education, Culture, and Science introduced the concept of a starting qualification (startkwalifikatie) to establish a minimum credential for young people that confers eligibility for the labor market. Those who do not attain a starting qualification are considered to be early school leavers. Diplomas from the precollege track (HAVO), preuniversity track (VWO), and upper secondary vocational education of at least two years (MBO at level two, which is the level of a basic skilled worker; see Traag and Van der Velden, 2011) all provide a starting qualification. Although compulsory education ends in the year that pupils turn 16 (leerplicht), they are obliged to stay in school until obtaining the starting qualification or turning 18 (kwalificatieplicht).

Figures 1.2 and 1.3 provide additional information on the completion rates in the different types of education over time. As Figure 1.2 shows, the share of students in the lower vocational education track, VBO, decreased sharply from the mid 1980s until 2007, from $41 \%$ to $26 \%$. The students' share completing a MAVO or HAVO track increased slightly, from on average $15 \%$ in the mid 1970 s to $21 \%$ in 2007 . A similar development, but on a lower level, is denoted for completion rates in the highest secondary track, VWO. In the same time period, the share of students with a VWO certificate increased from $10 \%$ to $16 \%$. At 


\section{Introduction}

postsecondary level, all tracks show a clear increase in enrolment and completion rates over time (see Figure 1.3). The students' share completing MBO rose largely from the mid 1970s until 2007 , from on average $14 \%$ to $43 \%$. The share of students graduating from vocational colleges (HBO) and universities (WO) increased in the same period from on average $10 \%$ to $26 \%$, and from on average $5 \%$ to $16 \%$, respectively.

Figure 1.2: Share of pupils with a final diploma in secondary education tracks, in the relevant age-range, for the school years 1974 to 2007 (3-year moving averages)

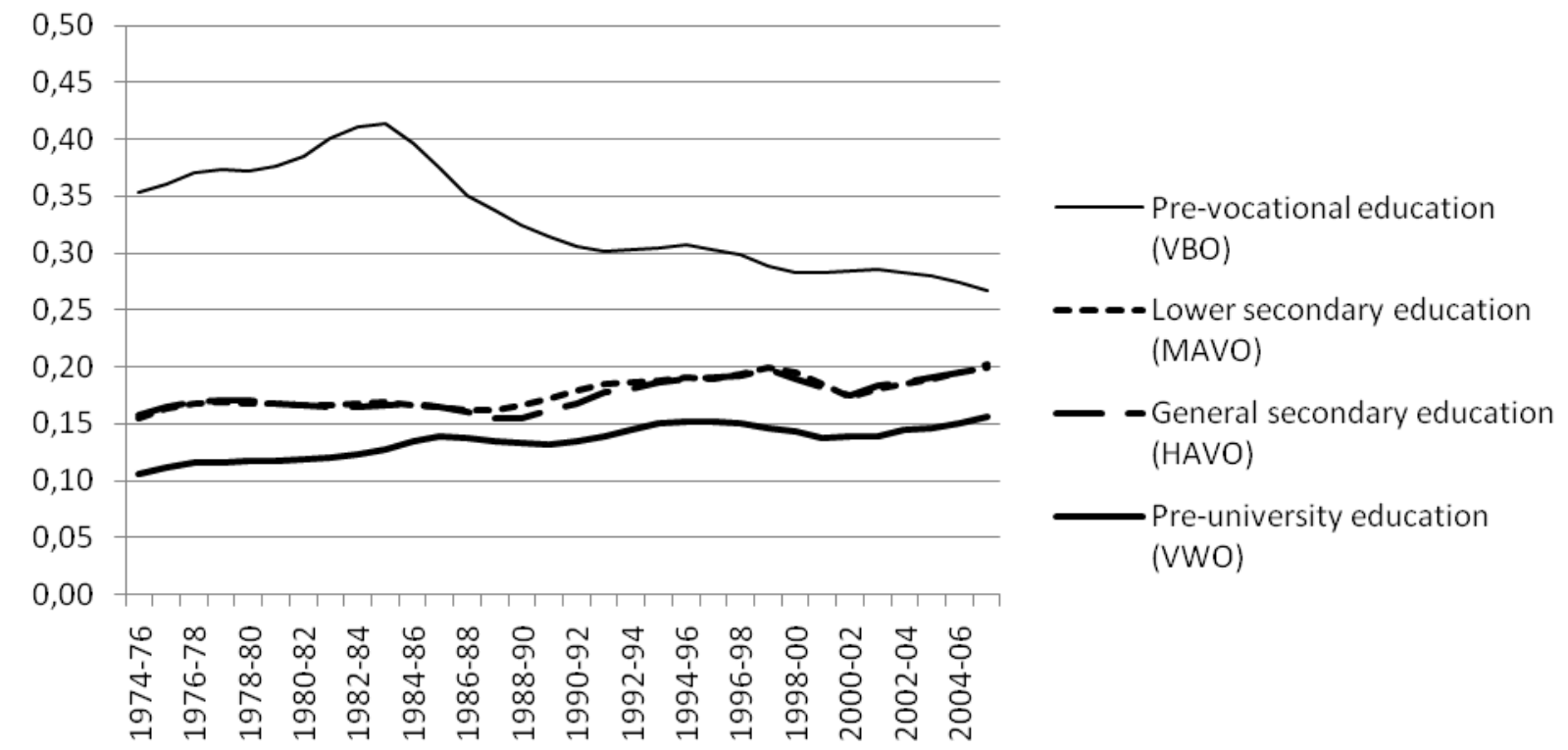

Source: CBS Statline, own calculations.

Note: The relevant age for those obtaining a lower vocational diploma (VBO) or lower general diploma (MAVO) is 16 years; for those obtaining a pre-college diploma (HAVO) it is 17 years and for those obtaining a pre-university diploma (VWO) it is 18 years. The share of pupils with a final diploma is calculated as follows: the total number of pupils with a lower vocational diploma (VBO) in each year is divided by the total population of 16 years-olds in each year. The same procedure is carried out for the total number of pupils with a diploma each year in either lower general track (MAVO), precollege track (HAVO) or pre-university track (VWO), which is then divided by the total population of 16,17 and 18 year-olds in each year, respectively. 
Figure 1.3: Share of pupils with a final diploma in postsecondary education tracks, in the relevant age-range, for the years 1974 to 2007 (3-year moving averages)

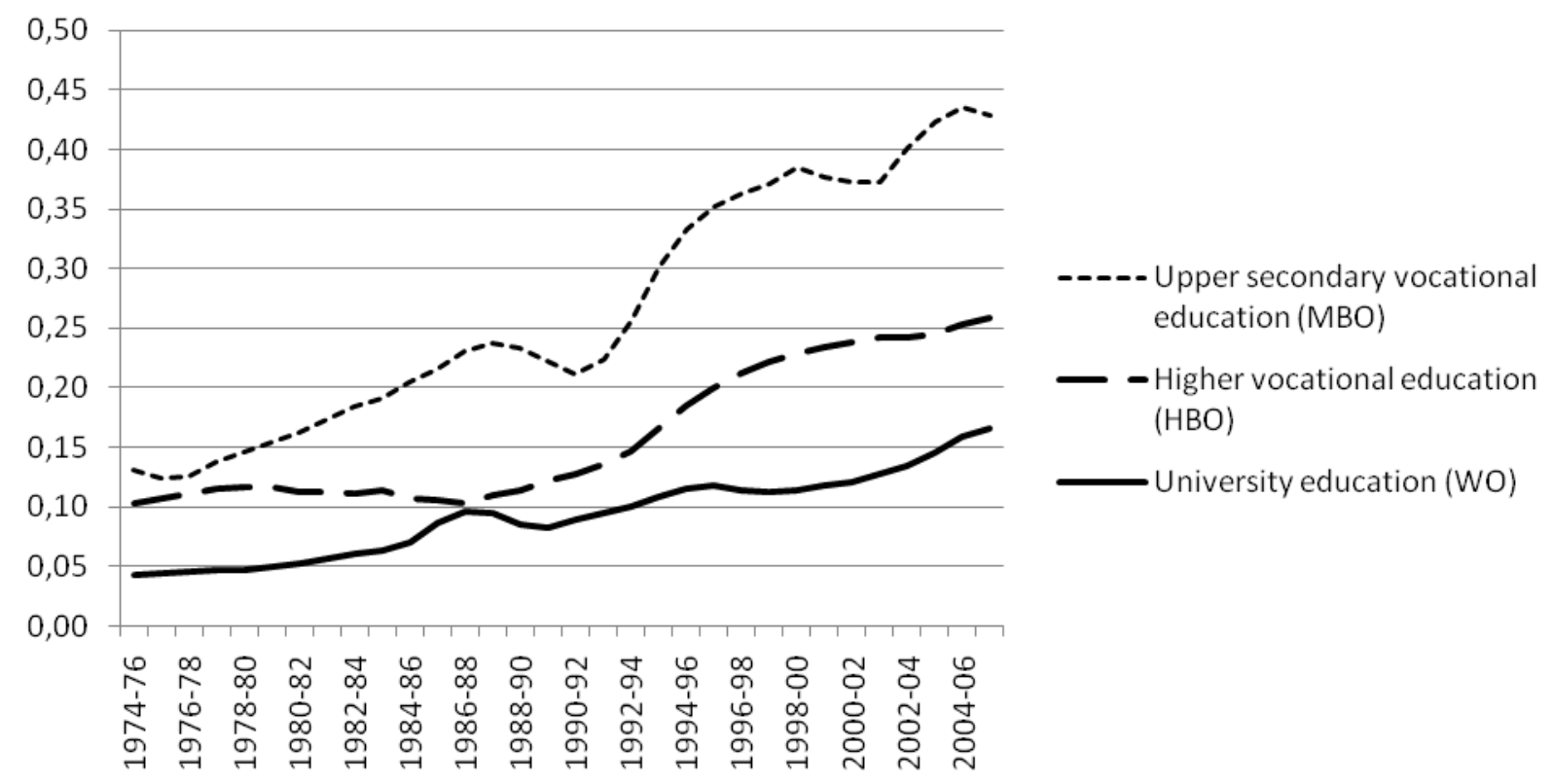

Source: CBS Statline, own calculations.

Note: The relevant age for those obtaining an upper secondary vocational diploma (MBO) is 20 years; for those obtaining a vocational college diploma (HBO) it is 22 years and for those obtaining a university diploma (WO) it is 23 years. The share of pupils with a final diploma is calculated as follows: the total number of pupils with an upper secondary vocational education diploma (MBO) in each year is divided by the total population of 20 year-olds in each year. The same procedure is carried out for the total number of pupils with a diploma each year in either vocational college (HBO) or university (WO), which is then divided by the total population of 22 and 23 year-olds in each year, respectively.

\subsection{Outline}

The remainder of this thesis is structured as follows: Chapter 2 introduces the theoretical concept of primary and secondary effects of social background. The related question addressed is: What is the relative importance of these effects in determining educational attainment, both at different transition stages of the educational career and over time in the Netherlands, and how does it determine the level of IEO? Chapter 3 follows closely the content of chapter 2, but focuses on the application of different estimation techniques to test for the robustness of previous findings in this field. Chapter 4 extends the view on labor market outcomes and addresses the question whether males and females are rewarded to the same extent for their cognitive skills, in general and conditional on education, and to what extent different study field choices thereby play a role. Chapter 5 explores how education, cognitive skills and further, social status-related mechanisms account for the intergenerational earnings persistence between fathers and sons. Chapter 6 summarizes the 
findings of each study and provides policy implications. Furthermore, chapter 6 discusses remaining issues and unanswered questions and presents suggestions for future research.

\section{Chapter 2. Direct and indirect effects of social background on educational attainment over time}

Social background is assumed to influence a child's educational choices and attainment indirectly by affecting performance in school, but also has a direct impact on educational choices. Boudon (1974) described this relationship as primary (indirect) and secondary (direct) effects of social stratification, which, in consequence, have important implications for a country's level of IEO. Based on this theoretical approach, Chapter 2 examines the relative importance of primary and secondary effects at the transition to higher secondary and postsecondary education and over four school cohorts in the Netherlands. We use the counterfactual method developed by Erikson et al. (2005) and Jackson et al. (2007) to disentangle performance-related differences from choice-related differences of social background. This approach was also used by Kloosterman, Ruiter, de Graaf, and Kraaykamp (2009), who studied the relationship between social background and the transition to higher secondary education at age 12 in the Netherlands. This chapter, however, also investigates the transition to higher postsecondary education at age 17 or 18; both conditional on the previous completion of higher secondary education at age 12, and unconditional of a higher secondary education degree, i.e. including all students making the transition to higher postsecondary education. Furthermore, we make an explicit distinction between the most and less prestigious routes in the higher tracks of secondary and tertiary education. We find secondary effects' relative importance decreases at the first transition over time, while it increases again at the second transition, which results in rather stable proportions of secondary effects over time in the conditional model. This is also supported by the fact that students from advantaged backgrounds more often choose straight career paths from the pre-university track to the university track. The unconditional model shows a moderate decrease in the relative size of secondary effects which is due to the decreasing relative importance of secondary effects at the first transition. In sum, the findings suggest that Dutch policy actions were mainly successful at the first transition but largely failed at the second transition in combating choice-related differences, and thus, in reducing IEO. 


\section{Chapter 3. Social background's effect on educational attainment: Methodological issues}

Chapter 3 investigates the changing impact of social background on children's educational outcomes over time and the educational career on the basis of the same theoretical approach as in chapter 2, but adds the following main aspects: First, with regard to data limitations and the decomposition structure applied in chapter 2, we assume that the findings are likely to be vulnerable to measurement error resulting in possible under- or overestimations of primary and secondary effects. Chapter 3 thus applies a counterfactual, a linear regression, and a structural equation model to test for the robustness of the findings. Second, social background effects are not only likely to alter at the transition stages, but also at the completion stages throughout the educational career (Boudon, 1974). The analysis therefore will be supplemented by estimating primary and secondary effects at the completion of secondary and postsecondary education levels, conditional on previous educational levels attended. Third, we include unconditional models on the total effect of social background on the highest educational level attained. The application of different estimation techniques and the use of conditional and unconditional models are related to the question whether a different handling of concepts and mechanisms has crucial impact on the relative importance of primary and secondary effects and thus on policy implications. The findings suggest that using different estimation methods matters with regard to the relative size of primary and secondary effects. The structural equation models show that secondary effects are overestimated at different stages of the educational career, when using counterfactual and linear regression models. We find secondary effects' relative size increases at postsecondary education levels and they are more important when completing a track than when entering a track. The size of IEO that is determined by choice-related differences thus reinforces at later stages in the educational career, which confirms assumptions of rational action and social reproduction theories, rather than of modernization theory. Moreover, the results obtained by the unconditional model on the overall importance of secondary effects do not yield major differences compared to the conditional model. This suggests that pathways through the Dutch education system are pretty straight forward, although detours are institutionally possible. 


\section{Chapter 4. Labor market returns to cognitive skills for males and females}

Chapter 4 analyses earnings returns to cognitive skills of males and females in the Netherlands, in general and conditional on their attained educational levels. The literature suggests that individuals are likely to sort into different labor markets according to their education (Heckman et al., 2006), while cognitive skills can contribute to additional differences in labor market success (e.g. Paglin \& Ruffalo, 1990; Murnane, Willet \& Levy, 1995; Arcidiacono, 2004). According to human capital theory, (investments in) schooling and skills are assumed to be equally rewarded for males and females at the labor market (Becker, 1964). However, studies on returns to cognitive skills for males and females show mixed results. We are particularly interested to what extent cognitive skills are related to males' and females' schooling and study field choices and may, in turn, account for differences in labor market outcomes. We use three proxies for measuring cognitive skills; two school achievement tests in math and language, and a test on non-verbal intelligence, all surveyed at age 12. Earnings information for the same individuals is measured at age 35 . We find significant earnings returns to cognitive skills, both for males and females. Specifically math skills are strong predictors for males' and females' earnings, but more for females, both in general and conditional on education. We attribute this result to the fact that cognitive skills better predict schooling outcomes for males than for females. Although males and females hardly differ in years of schooling, females' selection in education is less based on their cognitive skills. This suggests that females' educational success, more than for males, is related to other, unobserved factors, such as diligence and perseverance, while females' cognitive skills are fully recognized and rewarded at the labor market.

\section{Chapter 5. Transmission mechanisms accounting for intergenerational earnings persistence}

Chapter 5 examines four different transmission mechanisms through which a father's earnings affect a son's earnings; a son's educational attainment and cognitive skills, the family's cultural capital, and the social capital in the neighborhood. Research in this field argues that intergenerational income mobility is transmitted through the effects of parents' earnings on the child's educational attainment, which in turn affects the child's own earnings (Blanden, Gregg, and Macmillan, 2007). Higher income families thereby are better able to finance the educational costs of the offspring, which, in turn, reinforces the economic 


\section{Introduction}

advantages across generations (Becker and Tomes, 1979; Grawe, 2008). It is therefore likely that these transmission mechanisms account for intergenerational earnings persistence and implicitly determine the degree of social inequality in a society. Applying the conditional earnings mobility approach developed by Blanden et al. (2007), we estimate the extent to which these transmission mechanisms account for overall intergenerational earnings persistence in the Netherlands. While education and cognitive skills are conventional mechanisms, we additionally include the family's cultural capital and the social capital in the neighborhood as new transmission mechanisms to this approach. The findings show that a son's cognitive skills and schooling account for $50 \%$ of the father-son earnings elasticity. Education accounts for the largest part by far, while cognitive skills mainly work indirectly through educational attainment. This is much in line with the findings of Blanden et al. (2007) for the United Kingdom. The social capital of the neighborhood and the parents' cultural capital account for an additional $6 \%$ of the intergenerational income persistence. Of these two additional mechanisms, social capital appears to play the stronger role. As a robustness check, we test the model by replacing son's earnings with son's house property value, as a proxy for his permanent income. The results obtained thereby are virtually the same as in the original model. All transmission mechanisms together account for about $56 \%$ of the intergenerational earnings persistence, which raises the question what mechanisms are responsible for the remaining 44\%. Despite related measurement error, we suggest that part of it is connected to unobserved mechanisms, such as personality traits and spillover effects of family assets. 
2 How social background affects educational attainment over time in the Netherlands 


\subsection{Introduction}

There has been much research in the Netherlands on inequality of educational opportunity (IEO) for pupils of different socioeconomic backgrounds. ${ }^{1}$ Beginning with the seminal work of van Heek (1968), the focus of this research has mostly been on the transition that pupils face at age 12, the transition from primary to secondary education, with an overall conclusion that IEO in the Netherlands has decreased only slightly in recent decades (e.g., Bakker, Dronkers, and Schijf, 1982, 1986; Bakker and Cremers, 1994; Bakker and Schouten, 1991; De Graaf and Ganzeboom, 1993; De Jong, Dronkers, and Saris, 1982; Dronkers, 1983, 1993; Kloosterman et al., 2009; Peschar, Ten Vergert, and Popping, 1986; Sieben, Huinink, and de Graaf, 2001; Tieben, de Graaf, and de Graaf, 2010; Vrooman and Dronkers, 1986; Willemse, 1987). However, over a longer period, the Netherlands underwent a "process of opening-up and equalization” (Blossfeld and Shavit, 1993:18). A recent study by Buis (2010b) replicates the study of De Graaf and Ganzeboom (1993) but with improved data and modeling techniques. He shows that the trend toward decreasing IEO was most notable in the middle decades of the 20th century: for men, in the 1940s and 1950s, and for women, in the 1950s and 1960s. For more recent decades he found no significant trend. He also showed that at the beginning of the 20th century total IEO was mainly driven by inequalities in the transition from primary to secondary education, while toward the end of the century inequalities in the transition to postsecondary education become more relevant.

One problem with the above-mentioned studies that deal with long-term trends is that they lack data on children's academic performance. Few studies ask whether changes in the distribution of academic performance across social groups or changes in the propensity to make particular educational choices across social groups might have influenced IEO. However, disentangling performance-related differences from choice-related differences in educational attainment assumes two processes that are likely to determine IEO to a different extent over time and at each educational level. This is a concept based on Boudon's approach of primary and secondary effects of social stratification (Boudon, 1974), where IEO is understood as a consequence of both social background differences in academic performance (primary effects), and social background differences in educational choices that students make, conditional on their previous performance (secondary effects) (cf. Jackson, 2013: 2f.).

\footnotetext{
${ }^{1}$ This chapter is based on joint-work with Rolf van der Velden (for the modified, published version, see Büchner and Van der Velden, 2013).
} 
This chapter's aim is to investigate the relative importance of primary and secondary effects in creating IEO at two transitions points in the educational career and over four school cohorts in the Netherlands. The two transitions under study are the transition to higher secondary education at age 12 , and the transition to higher postsecondary education at age 17 or 18 .

For decomposing primary and secondary effects and analyzing their relative importance we use the methodological approach that is developed by Erikson et al. (2005) and Jackson et al. (2007), and is refined by Kartsonaki, Jackson, and Cox (2013). To our knowledge, only one study in the Dutch context used this approach to assess the relative importance of primary and secondary effects. Kloosterman et al. (2009) applied the methodological approach of Erikson et al. (2005) and Jackson et al. (2007) to examine the educational transition faced by Dutch pupils at age 12 (the transition from primary to secondary education), analyzing changes in primary and secondary effects over five birth cohorts. Using performance scores and parents' education to measure primary and secondary effects, they find that the secondary effects of social background have been more or less stable, while the primary effects have fluctuated (or somewhat increased) over the cohorts. Regarding the development of IEO, the results suggest that inequalities in the transition from primary to secondary education remained more or less stable over time.

Besides the study of Kloosterman et al. (2009), there are other studies that have analyzed the relationship between social background and the transition to higher postsecondary education in the Netherlands. They have found a positive correlation between higher social background and the propensity to make the transition to higher education (e.g., Bosma and Cremers, 1996; De Graaf and Ganzeboom, 1993; De Graaf and Wolbers, 2003; Rijken, Maas, and Ganzeboom, 2007; Kloosterman, 2010; Tieben and Wolbers, 2010). However, none of these studies considered possible changes in the relative importance of primary and secondary effects at this transition. ${ }^{2}$

By considering both the transition to higher secondary and postsecondary education, we can assess possible variation in IEO at different points of the educational career, which is not only a test of Boudon's assumptions, ${ }^{3}$ but also has important implications for policy measures. With regard to the institutional context and the (varying) level of IEO, we therefore include two more features in our analysis: First, we differentiate between the

\footnotetext{
${ }^{2}$ Although Kloosterman (2010) includes an analysis of the transition to postsecondary education in her doctoral dissertation, she focuses on upward and downward movements within tertiary education instead.

${ }^{3}$ Boudon states that "IEO rates are subject to variations as a function of national context, point in time, and school level. (...) [A] certain amount of IEO is present (...) at each school level” (1974:41).
} 
two highest tracks of secondary education (i.e. the precollege track and the pre-university track) and postsecondary education (i.e. the vocational college track and the university track), respectively. These tracks are conventionally seen as coequal in the Dutch education system, but the selection into these tracks is likely to be driven by social background. Second, the education system allows many back- and forward transitions within the secondary and postsecondary education level, which in principle gives all students the opportunity to repair erroneous track decisions. We therefore do not only look at the conditional transition probabilities from higher secondary to higher postsecondary education, but also analyze unconditional transition probabilities of all students making the transition to higher postsecondary education.

Our findings show that the relative importance of secondary effects decreases at the first transition at age 12 over time. Choice-related differences account for an average of $38 \%$ of total IEO at this transition. However, at the second transition at age 17 or 18 , secondary effects' relative importance increases again, resulting in rather stable proportions of secondary effects over time in the conditional model. This is also supported by the fact that students from advantaged backgrounds are more likely than students from lower social backgrounds to choose the straight and prestigious career paths from pre-university track to university. The unconditional model shows a moderate decrease in the relative size of secondary effects which is due to the decreasing relative importance of secondary effects at the first transition. In sum, our findings suggest that Dutch policy actions were mainly successful at the first transition but largely failed at the second transition in combating choice-related differences. Secondary effects still determine a large proportion of IEO and are driven, more than primary effects, by the features of the education system.

The remainder of this chapter is as follows: The next section provides an overview of the Dutch educational system and the distribution of students in educational tracks over time. Section 2.3 describes the methodological approach developed by Erikson et al. (2005) and Jackson et al. (2007) that we use for decomposing primary and secondary effects and subsequently assessing their relative importance. Section 2.4 introduces the data and variables operationalization and explains the model specifications. Section 2.5 presents the results. Section 2.6 summarizes the main findings and draws conclusions. 


\subsection{The Dutch school system}

As described in Chapter 1 and illustrated in Figure 1.1, the Dutch education system is highly stratified from secondary education onwards. The first transition, from primary to secondary education, takes place at age 12, where students can choose between two lower tracks (the lower general track, MAVO, and the lower vocational track, VBO) and two academic tracks (the precollege track, HAVO, and the pre-university track, VWO). The second transition, from secondary to higher postsecondary education, takes place at age 17 , for those entering vocational college (HBO), or at age 18 , for those entering university (WO). ${ }^{4}$

The two transition processes under study, the transition at age 12 and the transition at age 17 or 18, are quite different in terms of the underlying process. At the transition at age 12, pupils are selected mainly on the basis of their performance on the CITO test and their schoolteacher's recommendation. Although this seems to leave little room for nonmeritocratic factors, the secondary effects of social stratification do play a role. First, the teacher's advice is not only based on a pupil's past performance but also on motivational aspects and expected success in secondary education. The children of higher social classes are expected to be more successful and are consequently steered toward higher courses (Dronkers, 1993). Second, much of the advice is mixed (e.g., MAVO and HAVO, or HAVO and VWO), ${ }^{5}$ which leaves room for the parents to negotiate placement in the higher of the two tracks, an option that parents from higher social classes are usually keen to embrace. Finally, the management of secondary schools has autonomy when it comes to decisions about whether to admit pupils and can therefore diverge from test results or advice. This again opens up room for negotiation by the parents.

The transition to higher education at age 17 or 18 depends very much on attainment at the secondary education level. Having gained a diploma from the pre-university track (VWO), the student gains automatic access to university (WO), while a diploma from a precollege track (HAVO) gives automatic access to vocational college (HBO). Both types of diplomas are awarded on the basis of nationwide and schoolwide examinations. The national examinations ensure that the quality of diplomas is more or less similar nationwide. Some programs in higher education, however, may impose additional requirements on students (for example, access to higher-education programs in medicine and the arts is restricted). Although a pre-university track (VWO) diploma grants access to university (WO) and a

\footnotetext{
${ }^{4}$ For more description of the Dutch education system, see Chapter 1 , section 1.3.

${ }^{5}$ Teachers often give mixed recommendations for secondary school tracks if, for instance, the CITO test results are not clear-cut or if they diverge from the overall impression that a teacher has of the pupil
} 
precollege track (HAVO) diploma to vocational college (HBO), not every student will take that path. Some pre-university track (VWO) students choose the less prestigious vocational college (HBO) programs and some precollege track (HAVO) students choose an upper secondary vocational education (MBO) program that is not considered part of higher education. Alternatively, these students may decide not to study at all. As there are no entrance criteria other than having the required diploma from secondary education, we expect that the transition at age 17 or 18 to higher education is driven by secondary effects rather than by primary effects of social stratification.

\subsection{Methodology}

We analyze the relative importance of primary and secondary effects on the basis of the counterfactual $^{6}$ method of Erikson et al. (2005) and Jackson et al. (2007), which provides us with the opportunity to disentangle performance and choice in the context of socialbackground differences. We use this method for educational transitions both at age 12 and age 17 or 18.

The principle that underlies this methodological approach is that social background determines both the student's level of performance and the probability of choosing higher educational levels. Primary effects thereby can be related to the student's endowments, which operate indirectly, from social background to performance to transition. Secondary effects are related to student's preferences and operate directly, from social background to transition. The objective of this method is to disentangle the indirect effects from the direct effects and to estimate the relative strength of the two paths from social background to transition. Disentangling primary and secondary effects allows us to combine the choice distribution of students from one specific social background with the performance distribution of students from another social background (Kartsonaki et al., 2013:34f). In this way we produce counterfactual estimates that can be compared with the actual estimates for students who maintain the performance and choice distributions of their own social background. To give an example, we initially estimate the actual proportion of students from lower social background making the transition to higher education, conditional on their social background-specific performance and choice distribution. This proportion then will be compared with the

\footnotetext{
${ }^{6}$ The term counterfactual should not be confused with counterfactual analysis. Here, the term refers to the estimates that are obtained in contrast to the actual estimates by the method of Erikson et al. (2005) and Jackson et al. (2007). Alternatively, counterfactual estimates could be labeled as synthesized estimates (Kartsonaki et al., 2013).
} 
(hypothetical) proportion of students from lower social background making the transition to higher education, if they would have the performance distribution of students from higher social background, but keep the choice distribution of their own social background. Comparisons between the actual and counterfactual estimates build the basis to differentiate between the relative importance of primary and secondary effects.

Following this principle, we categorize students by their social background and divide them into three groups of low, medium, and high social background (for detailed variable description, see section 2.4). Their performance scores are assumed to be normally distributed $^{7}$ within each social background group, while their choice of making the transition to higher education is assumed to be binary distributed. Thus, within each social background group, the individual's probability of making the transition, given performance $x$, can be expressed as

$$
\int \sigma^{-1} \phi\left(\frac{x-\mu}{\sigma}\right) \Lambda(\alpha+\beta x) d x
$$

where performance $x$ follows a normal distribution with mean $\mu$ and standard deviation $\sigma^{2}$ (that is, $x \sim N\left(\mu, \sigma^{2}\right)$ ) and $\phi(\cdot)$ denotes the standard normal density function. $\Lambda(\cdot)$ is the logistic function, with $\alpha$ and $\beta$ as the parameters of the logistic curve, denoting the conditional transition probability at any specific performance x (Erikson et al., 2005; Kartsonaki et al., 2013). ${ }^{8}$

Now it is possible to estimate the relative importance of primary and secondary effects in the transition gap between different social background groups. Therefore, we divide the counterfactual odds ratios by the factual odds ratios and vice versa (Erikson et al., 2005): Assume $P_{j k}$ is the probability of an individual making the transition with the performance distribution of social background group $j$ and the choice distribution of social background group $k$. The odds ratio for the transition propensity $P$ between $j$ and $k$ then can be written as

$$
Q_{j j . k k}=\left(P_{j j} /\left(1-P_{j j}\right)\right) /\left(P_{k k} /\left(1-P_{k k}\right)\right)
$$

\footnotetext{
${ }^{7}$ Buis (2010a) shows that the assumption of normally distributed performance scores can be relaxed and replaced by the empirical distribution instead.

8 Jackson et al. (2007) propose to calculate the integral by numerical integration that allows factual and counterfactual combinations of background-specific performance distributions with performance-specific transition propensities of any other social background group.
} 
To obtain counterfactual odds ratios, we now replace either the performance distribution or the choice distribution of social background group $j$ by that of $k$. That is, when isolating primary effects the conditional transition propensity of social background group $k$ will be replaced by that of social background group $j$ :

$$
Q_{j j . k j}=\left(P_{j j} /\left(1-P_{j j}\right)\right) /\left(P_{k j} /\left(1-P_{k j}\right)\right)
$$

$Q_{j j . k j}$ denotes the odds ratio, where choice characteristics are the same while the remaining difference in the odds between the two groups is due to performance, thus describing primary effects (Kartsonaki et al., 2013). Respectively, to isolate secondary effects we replace the performance distribution of social background group $k$ :

$$
Q_{j j . j k}=\left(P_{j j} /\left(1-P_{j j}\right)\right) /\left(P_{j k} /\left(1-P_{j k}\right)\right)
$$

Taking the logarithms of the odds ratios, the relative importance of secondary effects then can be written as

$$
\begin{array}{cc} 
& L_{j j . j k} / L_{j j . k k} \\
\text { or } \quad & L_{k j . k k} / L_{j j . k k} .
\end{array}
$$

The relative importance of primary effects then is

$$
\begin{array}{ll} 
& 1-\left(L_{j j . j k} / L_{j j . k k}\right) \\
\text { or } \quad & 1-\left(L_{k j . k k} / L_{j j . k k}\right) .
\end{array}
$$

It is possible that (2.5) and (2.6) produce slightly deviating results. Jackson et al. (2007) therefore propose to take the average of the two estimates. ${ }^{9}$

\footnotetext{
${ }^{9}$ Kartsonaki et al. (2013) point out that "[t]his method provides useful estimates only if the log odds (...) have the same sign." (ibid:39). The authors also propose to calculate standard errors to assess whether the estimates are correctly measured between different social background groups and over time. Further issues and some general concern with this method is related to measurement error; for example, due to the operationalization and assessment of primary effects or possible correlations between primary and secondary effects (e.g. Jackson et al., 2007; Morgan, Spiller, and Todd, 2013; Schindler, 2010). However, despite the limitations, the method provides some useful tools to comprehend the mechanisms that underlie the process of IEO.
} 


\subsection{Data and model specification}

Our analysis is based on panel datasets of four school cohorts provided by Statistics Netherlands (CBS). Each dataset is a nationally representative sample, starting in $1977(N=$ 37,280), 1982 ( $N=16,813), 1989(N=19,524)$, and $1993(N=20,331)$ respectively. The pupils in each cohort are followed from age 12, when they enter secondary education, until the end of their full-time education or until age of 28. Each dataset provides comparable information on the socioeconomic background and standardized skills tests of pupils at the entry to secondary education. School track choices and whether a final examination was passed are documented by the schools participating in the CBS survey and are provided each year for all students. ${ }^{10}$

Our measure of social background is based on the highest educational level achieved by the parents. Most studies examining primary and secondary effects use parents' occupational information as proxy for social background (e.g. Jackson 2013). The occupational information in our data though does not allow differentiation into social classes as conventionally defined. However, as demonstrated by several Dutch studies (e.g., Bakker and Cremers, 1994; De Graaf and Ganzeboom, 1993), the impact of father's occupational status on children's educational attainment appears to have decreased over time, while father's educational level is a much stronger and more stable predictor of children's educational attainment. This finding supports the assumption that the Netherlands, compared to other European countries (e.g., England), is a "knowledge-based" society rather than a “class” society (Kraaykamp, Van Eijck, and Ultee, 2010). The lack of social-class measures in our data, however, means that we cannot compare the role of social class and parents' education in educational inequality, nor can we consider the role of social class in determining the relative importance of primary and secondary effects.

\subsubsection{Dependent variables}

As discussed above, we aim to investigate the relative importance of primary and secondary effects over time at two stages of the educational career: the transition to secondary education, when pupils are 12 years old, and the transition to higher education, when pupils are age 17 or 18 . Figure 2.1 illustrates the transitions in the Dutch school system for the 1993

\footnotetext{
${ }^{10}$ A student is understood to have successfully completed an educational track if (s)he passed the final examination. If a student dropped out before finishing a grade, we consider the most recent available information about his or her educational transitions
} 
cohort and shows the transition and completion rates for the secondary and postsecondary levels. The proportions of students making the transitions to the different tracks in secondary education have hardly changed over time. ${ }^{11}$ Around $30 \%$ of all pupils of each cohort go to one of the higher tracks (the precollege track HAVO or the pre-university track VWO). The vast majority of them also complete their chosen track. The share of pupils completing a precollege track is even higher than the share of pupils entering the track, because of upward movements of pupils from the lower general track (MAVO) (see percentages in Figure 2.1).

Figure 2.1: Transition and completion rates in secondary and postsecondary education, 1993 school cohort

$\begin{array}{llll}\text { Age } & 12 & 17-18 & 21-22\end{array}$

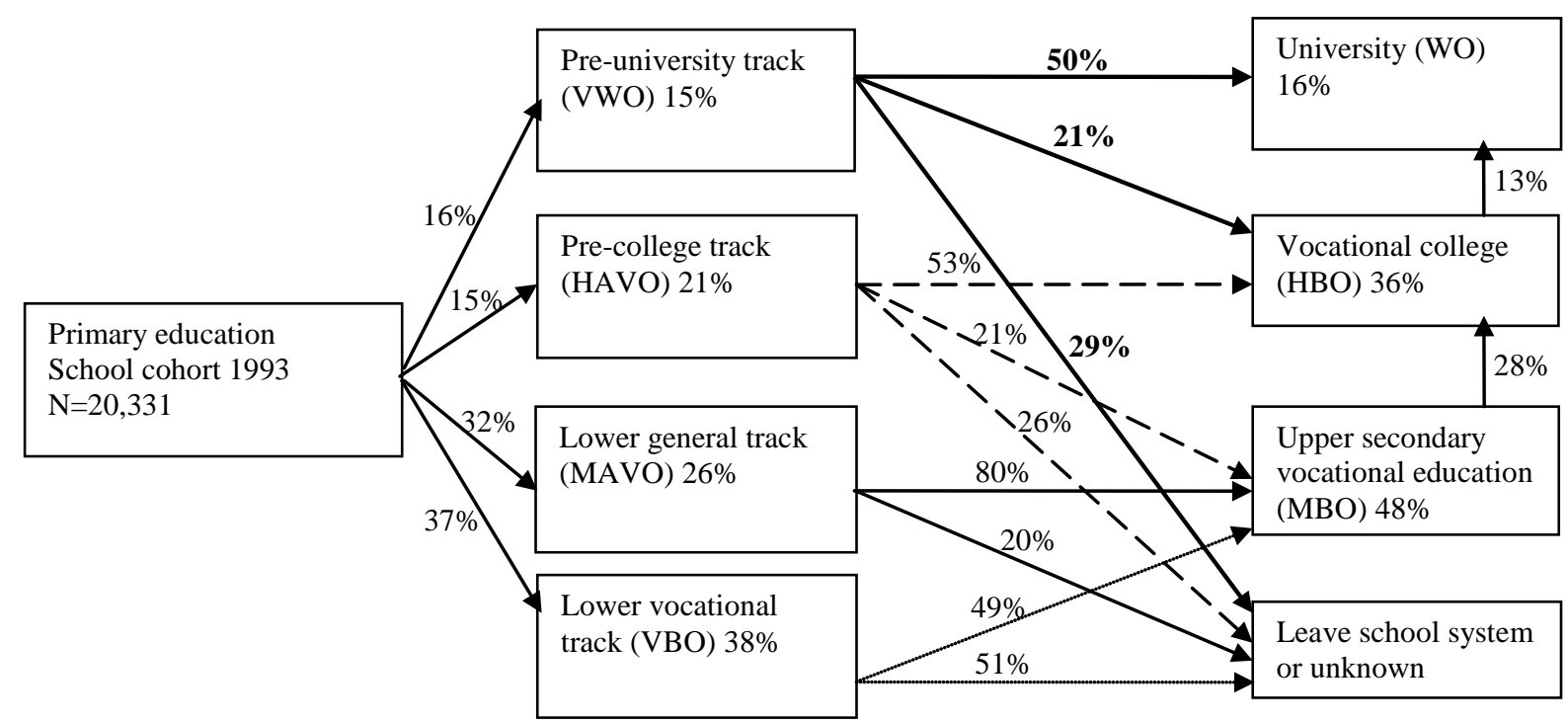

Source: CBS cohort 1993; own calculations.

Note: The arrows represent the transition from one track to another track and add up to $100 \%$. The figures in the boxes represent the highest completed level of secondary or postsecondary education. These can differ from the inflow as a result of up- or downward mobility. For the transition at age 12 we have information for all students. For the transition at age 17-18 we lack information for students who dropped out of the panel or who left school altogether; they are in the category "Leave school system or unknown."

Clearer changes over time occur at the transition to postsecondary education (see Table 2.1). The share of pupils from the pre-university track (VWO) entering university (WO) increased from 45\% in the 1977 cohort to 50\% in the 1993 cohort. The largest increase occurred with the transition to vocational colleges (HBO). The rates of transition from the precollege track (HAVO) to vocational college continuously increased from 31\% (1977 cohort, see Figure A2.1 in the appendix) to 53\% (1993 cohort, see Figure 2.1). The growing

\footnotetext{
${ }^{11}$ Corresponding figures for the other three cohorts are presented in Figures A2.1-A2.3 in the appendix.
} 
popularity of vocational college is also evident from completion rates and transfers to this track. In the 1977 cohort 26\% of all pupils obtained a vocational college certificate. Included in this group are $11 \%$ of those who entered upper secondary vocational education (MBO), later transferring to vocational college. By the 1993 cohort the number of vocational college graduates had increased to $36 \%$ and transfers from upper secondary vocational education (MBO) to the vocational college certificate continuously increased over time to reach $28 \%$ (see Figure 2.1 and Figures A2.1, A2.2 and A2.3 in the appendix).

Table 2.1: Descriptive statistics (\%)

\begin{tabular}{lcccc}
\hline & 1977 & 1982 & 1989 & 1993 \\
\hline $\begin{array}{l}\text { Parents' education } \\
\text { Low }\end{array}$ & 53 & 51 & 44 & 30 \\
$\quad$ Medium & 31 & 29 & 36 & 45 \\
$\quad$ High & 16 & 20 & 20 & 25 \\
& & & & \\
Transition at age 12 to precollege track (HAVO) or & 29 & 31 & 28 & 31 \\
preuniversity track (VWO) & 17 & 17 & 15 & 16 \\
Transition at age 12 to preuniversity track (VWO) & & & & \\
Transition at age 17-18 from precollege track (HAVO) \\
$\begin{array}{l}\text { or preuniversity track (VWO) to vocational college } \\
\text { (HBO)/university (WO) }\end{array}$ & 49 & 57 & 60 & 60 \\
$\begin{array}{l}\text { Transition at 17-18 from preuniversity track (VWO) to } \\
\text { university (WO) }\end{array}$ & 45 & 54 & 48 & 50 \\
$\begin{array}{l}\text { Unconditional transition of all to vocational college } \\
\text { (HBO) or university (WO) }\end{array}$ & 20 & 21 & 20 & 22 \\
Unconditional transition of all to university (WO) & 8 & 10 & 7 & 8 \\
\hline$N$ at age 12 & 37,050 & 7,864 & 19,287 & 19,994 \\
$N$ at age 17-18 & 18,123 & 4,242 & 12,193 & 11,965 \\
\hline
\end{tabular}

Source: CBS cohorts 1977, 1982, 1989, 1993; own calculations.

In constructing our measure of the transition at age 12, we consider two definitions that could be considered appropriate in the Dutch case. First, we look at only pupils entering the pre-university track (VWO), and then —in line with Kloosterman et al. (2009) —we consider pupils entering precollege track (HAVO) or pre-university track (VWO). We think that this is a crucial distinction in the Dutch school track system, since the pre-university track is typically considered to be an elite track, while the precollege track is usually regarded as a track for talented students from the intermediate and lower social classes. Combining these two tracks is likely to lead to an underestimation of IEO. The same type of distinction applies at the transition to higher education: the vocational track in higher education (HBO) 
has absorbed much of the increase in enrollment rates, so that vocational college typically has the status of mass education, while the university (WO) maintained its status of elite education.

Summing up, in the analyses we use the following model specifications: ${ }^{12}$

1. The probability of entering either precollege track (HAVO) or pre-university track (VWO)

2. The probability of entering pre-university track (VWO)

3. The conditional probability of making the transition to vocational college (HBO) or university (WO) given a diploma from a precollege track (HAVO) or a pre-university track (VWO)

4. The conditional probability of making the transition to university (WO) given a diploma from a pre-university track (VWO)

5. The unconditional probability of all students making the transition to vocational college (HBO) or university (WO)

6. The unconditional probability of all students making the transition to university (WO)

\subsubsection{Independent variables}

Social Background. As a measure of social background we use the highest educational level achieved by either the father or the mother. We distinguish between low educated (primary education, lower vocational track VBO or lower general track MAVO), medium educated (precollege track HAVO, pre-university track VWO, or upper secondary vocational education MBO), and high educated (vocational college HBO or university WO). ${ }^{13}$

The upper part of Table 2.1 shows that the share of low educated parents decreased over time over the cohorts, while the shares of medium and high educated parents clearly increased over time. Cross-tabulating parents' educational attainment with the child's first transition at age 12 shows that the relationship between social background and educational choices is rather stable over time (see Table A2.1 in the appendix). In every cohort, some $60 \%$ of children from high educated households continue to precollege track (HAVO) or preuniversity track (VWO), while the corresponding figures for children from households with low educated parents are 14-19\% across the cohorts.

\footnotetext{
${ }^{12}$ Each of the transition variables is binary, taking a value of 1 when making the transition and 0 otherwise. In the case of mixed tracks in the orientation years of secondary education, we look at the first clear track decision after the orientation phase (cf. Kloosterman et al., 2009:383).

${ }^{13}$ In the CBS survey of the 1982 cohort, information on parents' education was collected differently from the surveys of the other three cohorts. This may cause some distinct deviations from a trend when we compare results over time.
} 
Academic Performance. As a measure of academic performance we use the average performance scores taken from arithmetic and language tests at age 12. The tests in the four surveys are based on a nationwide compulsory test that is developed by CITO. These compulsory tests are usually conducted at the end of primary education and estimate the pupil's level of school-based knowledge, in line with national curriculum requirements. The test scores in the surveys are derived from a shortened version of the national CITO tests, ${ }^{14}$ which we have standardized to have a mean of 0 and a standard deviation of 1 . These scores are used as indicators of the performance level of the students at the beginning of secondary education. The test results in the data are comparable with those of the real CITO tests at the end of primary education (Hustinx, Kuyper, Van der Werf, and Zijsling, 2005). ${ }^{15}$

Measuring performance before the transition at age 17 or 18 to higher education is more challenging. It is not possible to use grades as measures of performance, because grades are specific to the different tracks in secondary education and therefore cannot be compared across tracks. Furthermore, test scores at age 17 or 18 are also unavailable, because the CITO test is administered only once in the survey, at age 12. Given these constraints, we are therefore forced to use the age 12 CITO test results in our analyses of the transition at age 17 or 18. It is possible to check the effect of using early test scores rather than scores measured close to the transition. For two cohorts (the 1989 and the 1993 cohorts), an additional test in mathematics and language was constructed by CITO and carried out in the third year of the cohort study, at around age 15 . We will therefore repeat some analyses of primary and secondary effects using the test scores at age $15 .^{16}$

Table 2.2 displays the average performance scores of the pupils at age 12 across the four cohorts. Pupils with high educated parents perform best out of the three groups, with an

\footnotetext{
${ }^{14}$ Pupils in the survey thus complete these tests twice; once, officially, at the end of primary education and once as a respondent in the survey. Choices for subsequent school tracks have already been made on the basis of the regular CITO test. In contrast to the 1977, 1989, and 1993 cohorts, the 1982 cohort took the survey-specific CITO test at the same time as the real CITO test, i.e., one year earlier. This leads to comparably lower performance scores in the survey for pupils of the 1982 cohort (CBS, 1992).

${ }^{15}$ According to Hustinx et al. (2005), the Cronbach alpha of the CITO tests in the 1977 and 1982 school cohorts is 0.80 .

${ }^{16}$ The use of the second test is unfortunately still problematic, due to three factors. First, as a result of the design of the data collection, pupils who had to repeat classes at any stage before the second test did not participate in this test. Second, the tests were not the same for each pupil. There was a relatively simple test for those who at age 15 were in the lower general track (MAVO) or the lower vocational track (VBO) and another, more difficult, test for pupils in the precollege track (HAVO) and the preuniversity track (VWO). Although some recalibration has taken place, the resulting test scores do not provide a perfect estimate of performance at age 15. Third, since the higher-education track is chosen at age 17 or 18, the second test at age 15 cannot provide a perfect proxy for pupils' performance at this later transition. Given these restrictions, the second test will not guarantee a completely unbiased comparison between performance and choice at the transition to higher education across different social backgrounds.
} 
average standardized score of 0.59. Pupils with low educated parents have the lowest performance scores of the three groups. Over time, the average level of performance of children with low and medium educated parents slightly decreased, while the average scores for children with high educated parents remained more or less stable. The average performance scores in the second test at age 15, for the 1989 and 1993 cohorts, do not differ much from those at age 12. Some deviations can be found for pupils with high educated parents; in the 1989 cohort, 15-year-olds performed slightly better than at age 12, while they did worse at 15 than at 12 in the 1993 cohort (see Table A2.2 in the appendix).

Table 2.2: Means and standard deviations (in parentheses) of standardized performance scores at age 12, by school cohort

\begin{tabular}{lcccccc}
\hline \multicolumn{1}{c}{1977} & \multicolumn{2}{c}{1982} & 1989 & 1993 \\
\hline Parents' education & & & & & & \\
Low & $-0.22(0.971)$ & $-0.26(0.937)$ & $-0.31(0.965)$ & $-0.36(0.942)$ \\
Medium & $0.14(0.947)$ & $0.15(0.961)$ & $0.10(0.931)$ & $0.06 \quad(0.931)$ \\
High & $0.59(0.890)$ & $0.53(0.955)$ & $0.60(0.872)$ & $0.60 \quad(0.857)$ \\
\hline
\end{tabular}

Source: CBS cohorts 1977, 1982, 1989, 1993; own calculations.

\subsection{Results}

Before discussing the estimated relative importance of primary and secondary effects, we present the factual and counterfactual proportions of students making each transition.

\subsubsection{Transition at age 12}

The diagonal numbers from the upper left corner to the lower right corner for each cohort in Table 2.3 shows the actual probabilities of students from each social-background group transitioning to pre-university track (VWO) and to pre-college (HAVO) or pre-university tracks (VWO). As a general observation we can note that the actual transition probabilities for all children have decreased by 3-4 percentage points over time. In our reference cohort (1993), $6 \%$ of students with low educated parents, $14 \%$ of students with medium educated parents, and $40 \%$ of students with high educated parents proceeded to the pre-university track (VWO). All other numbers in the table show counterfactual proportions. To give an example, if in the 1993 cohort students from low educated parents had the average performance of students with high educated parents but had kept their own choice behavior constant, their 
probability of entering the pre-university track would have increased from $6 \%$ to $21 \%$. In contrast, if students from low educated parents had the choice behavior of the students with high educated parents but kept their own performance constant, their probability of entering the pre-university track would have increased from $6 \%$ to $14 \%$.

If we look at the counterfactual proportions in Table 2.3, we can make two observations. First, for students from low educated backgrounds in the 1977 cohort, whether they adopted the performance level or adopted the choice behavior of students from high educated parents made little difference: both cases would lead to an increase in the probability of making the transition to the pre-university track from the (actual) rate of $9 \%$ to a (counterfactual) transition probability of 22\% or 21\%, respectively. In the 1993 cohort, however, these same transition propensities changed from $6 \%$ to $21 \%$ and $14 \%$, respectively. That is, the performance-related differences between children from low and high educated parents increased over time, while the choice-related differences (with the exception of the 1982 cohort) remained more or less constant.

Table A2.3 in the appendix shows results for a similar analysis of entry to precollege track (HAVO) or pre-university track (VWO). Students with high educated parents have the highest probability of all groups over time to proceed to one of these tracks, while the probability of going to precollege track or pre-university track for students with low and medium educated parents is half to a quarter of that and decreasing over time. In the 1993 cohort, $15 \%$ of the students with low, $30 \%$ with medium, and $64 \%$ with high educated parents proceeded to either precollege track or pre-university track. Note that, regardless of changes over time, the probability of going to precollege track or pre-university track, rather than to pre-university track only, is about twice as high for students with low and medium educated parents and about 1.5 times higher for students with high educated parents. It suggests that the precollege track provides a crucial opportunity for students with low and medium educated parents to proceed to higher education. Again we can note that the performance-related differences between those with low and high educated parents have increased over time (especially between the first two and the last two cohorts), while the choice-related differences have stayed more or less the same.

\subsubsection{Primary and secondary effects at the age 12 transition}

Table 2.4 confirms these findings and shows that secondary effects on average declined in importance over time at the transition at age 12 (see also Figures A2.4 and A2.5 in the appendix). 
2 How social background affects educational attainment over time in the Netherlands

Table 2.3: Estimated factual and counterfactual transition rates (\%), from primary education to pre-university track (VWO), age 12

\begin{tabular}{|c|c|c|c|c|c|c|c|c|c|c|c|c|c|}
\hline & & \multicolumn{3}{|c|}{1977} & \multicolumn{3}{|c|}{1982} & \multicolumn{3}{|c|}{1989} & \multicolumn{3}{|c|}{1993} \\
\hline & & \multicolumn{3}{|c|}{ Choice } & \multicolumn{3}{|c|}{ Choice } & \multicolumn{3}{|c|}{ Choice } & \multicolumn{3}{|c|}{ Choice } \\
\hline & & Low & Medium & High & Low & Medium & High & Low & Medium & High & Low & Medium & High \\
\hline \multirow[t]{3}{*}{ Performance } & Low & 9 & 12 & 21 & 7 & 11 & 20 & 6 & 8 & 14 & 6 & 8 & 14 \\
\hline & Medium & 13 & 18 & 29 & 12 & 18 & 30 & 10 & 13 & 22 & 11 & 14 & 23 \\
\hline & High & 22 & 29 & 43 & 18 & 26 & 40 & 18 & 23 & 37 & 21 & 26 & 40 \\
\hline
\end{tabular}

Source: CBS cohorts 1977, 1982, 1989, 1993; our calculations.

Table 2.4: Relative importance (\%) of secondary effects at age-12 transition from primary education to (1) pre-university track (VWO) and (2) precollege track (HAVO) or pre-university track (VWO)

\begin{tabular}{|c|c|c|c|c|c|c|c|c|}
\hline & \multicolumn{2}{|c|}{1977} & \multicolumn{2}{|c|}{1982} & \multicolumn{2}{|c|}{1989} & \multicolumn{2}{|c|}{1993} \\
\hline & $(1)$ & $(2)$ & (1) & $(2)$ & $(1)$ & $(2)$ & $(1)$ & $(2)$ \\
\hline Medium-low & 45 & 40 & 46 & 47 & 34 & 36 & 30 & 34 \\
\hline High-low & 48 & 44 & 52 & 53 & 43 & 41 & 39 & 40 \\
\hline High-medium & 50 & 47 & 57 & 58 & 48 & 45 & 44 & 44 \\
\hline Average & 48 & 44 & 52 & 53 & 42 & 41 & 38 & 39 \\
\hline
\end{tabular}

Source: CBS cohorts 1977, 1982, 1989, 1993; our calculations. 
Between the first two cohorts and the last two cohorts, the relative importance of secondary effects at the transition to the pre-university track (VWO) decreased from around $50 \%$ to around $40 \%$. For the transition to either precollege track (HAVO) or pre-university track (VWO), the percentages fluctuated a little more (with a clear outlier in the 1982 cohort), but we also note a (smaller) decrease from 44\% in the 1977 cohort to 39\% in the 1993 cohort. The relative importance of secondary effects over all cohorts is thus on average $45 \%$ at the transition to pre-university track and $44 \%$ at the transition to precollege track or preuniversity track. Primary effects are on average slightly more important at this transition. Interestingly, the relative importance of secondary effects is highest when explaining the difference between students with medium and high educated parents.

The results we have obtained are, with small deviations, in line with those of Kloosterman et al. (2009) for the same cohorts. When we consider the 1977, 1989, and 1993 cohorts, the relative importance of primary effects increased between students from medium and low, high and low, and high and medium educated parents over time. The 1982 cohort interrupts this linear trend, and we observe a decrease in the relative importance of primary effects from 1977 to 1982 . Summarizing our findings, we can say that the primary effects outweigh the secondary effects at the transition at age 12, but that nonetheless secondary effects are still substantial, even if their relative share appears to decrease over time.

\subsubsection{The conditional transition at age 17 or 18}

Compared to the first transition at age 12, the actual transition rates at age 17 or 18 from preuniversity track (VWO) to university (WO) and from precollege (HAVO) or pre-university (VWO) tracks to vocational college (HBO) or to university (WO) of students with low, medium, and high educated parents very clearly increased over time. The selection process at the first transition already functions as an important selection mechanism for the future transition into higher education. As Table 2.5 shows, students with low educated parents who graduated in the pre-university track increased their probability of making the transition to university from 34\% in the 1977 cohort to $42 \%$ in the 1993 cohort. Students with medium and high educated parents had generally higher, but-with the exception of the 1982 cohort - rather constant transition rates over time (with medium educated parents, $43 \%$ in the 1977 cohort and 46\% in the 1993 cohort; with high educated parents, 54\% in the 1977 cohort and 55\% in the 1993 cohort). The increase in actual transition rates for those from low educated households is even clearer when looking at the transition to vocational college or to 
university (see Table A2.4 in the appendix). The expansion of Dutch higher education, mostly due to an increase in enrollment in vocational colleges, facilitated access to higher education over time for students with low and medium educated parents.

The counterfactual proportions in the off-diagonal numbers in Table 2.5 reveal that secondary effects are much stronger than primary effects at the transition at age 17 or 18 (see also Table A2.4 in the appendix). Table 2.5, for example, shows for the 1993 cohort that if students with low educated parents keep their own performance distribution but have the transition propensity of students from high educated parents, their transition rate to university would be $54 \%$ (actual transition rate is $42 \%$ ). The opposite is reported for students from high educated parents: if their average performance distribution is combined with the transition propensity of students with low educated parents, their transition rate to university would be $42 \%$ (actual transition rate is $55 \%$ ). Differences in performance (vertical differences) are practically nonexistent at this transition. All differences between students from different educational backgrounds are therefore driven by secondary effects (horizontal differences). This is also true for the transition to vocational college or university (see Table A2.4 in the appendix), supporting the argument that there are few differences in performance between students from different social backgrounds at this transition (no primary effects), but there are different preferences in choosing higher education (secondary effects).

To sum up, primary effects at the transition at age 17 or 18 are quite small and change very little over time. The secondary effects are larger (although not as large as at the first transition at age 12) and explain most of the differences in observed social-background effects, although these effects clearly decrease over time.

\subsubsection{The unconditional transition at age 17 or 18}

An unconditional analysis, that is, of all pupils to university (WO) and all pupils to vocational college (HBO) or university (WO), allows us to take into account the possibility of upward movements through alternative tracking paths in the Dutch educational system and thus provides a picture of overall IEO at this transition stage. As Table 2.1 illustrates, students are less likely to enter vocational college (HBO) or university (WO) in the unconditional model than in the conditional model, which suggests that students move on rather straight career paths. In the 1993 cohort, for instance, 50\% of the students who graduated in the preuniversity track (VWO) made the transition to university, while only $8 \%$ did so when alternative tracking paths are considered. 
Table 2.5: Estimated factual and counterfactual transition rates (\%), from pre-university track (VWO) to university (WO), conditional transition at age 17 or 18

\begin{tabular}{|c|c|c|c|c|c|c|c|c|c|c|c|c|c|}
\hline & & \multicolumn{3}{|c|}{1977} & \multicolumn{3}{|c|}{1982} & \multicolumn{3}{|c|}{1989} & \multicolumn{3}{|c|}{1993} \\
\hline & & \multicolumn{3}{|c|}{ Choice } & \multicolumn{3}{|c|}{ Choice } & \multicolumn{3}{|c|}{ Choice } & \multicolumn{3}{|c|}{ Choice } \\
\hline & & Low & Medium & High & Low & Medium & High & Low & Medium & High & Low & Medium & High \\
\hline \multirow[t]{3}{*}{ Performance } & Low & 34 & 42 & 53 & 42 & 50 & 62 & 41 & 44 & 52 & 42 & 46 & 54 \\
\hline & Medium & 35 & 43 & 53 & 42 & 51 & 62 & 41 & 44 & 53 & 42 & 46 & 55 \\
\hline & High & 36 & 43 & 54 & 42 & 51 & 63 & 42 & 34 & 54 & 42 & 46 & 55 \\
\hline
\end{tabular}

Source: CBS cohorts 1977, 1982, 1989, 1993; our calculations.

Table 2.6: Estimated factual and counterfactual transition rates (\%) of all students to university (WO), unconditional transition at age 17 or 18

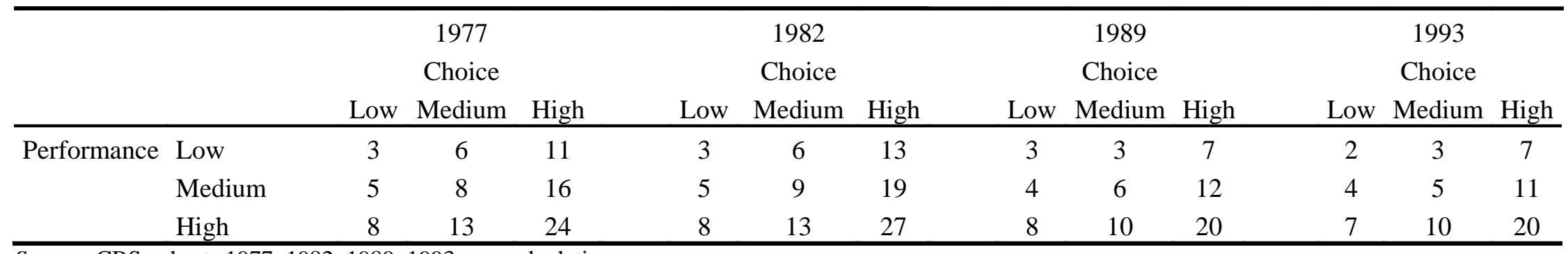

Source: CBS cohorts 1977, 1982, 1989, 1993; our calculations. 
However, if we consider the transition to both vocational college and university, the share of students proceeding to higher-education tracks at the transition at age 17 or 18 clearly increases compared to the option to enroll only in university. A relatively constant share over time, of about $21 \%$ of the students coming from different school tracks, proceeds to either vocational college or university, while the average share proceeding to university is about $8 \%$ over time.

As would be expected, Table 2.6 shows that the factual and counterfactual proportions in the unconditional model are much lower than in the conditional model (see Table A2.5 in the appendix for equivalent proportions for the unconditional transition to vocational college or university). The factual transition probabilities to university and to vocational college or university for students with low, medium, and high educated parents are low and even decreasing over time. Across the cohorts, the unconditional transition probability of students with the average performance and transition propensity of those with low educated parents is $3 \%$ to university and $10 \%$ to vocational college or university. Students with medium educated parents have a transition probability of $7 \%$ to university and $21 \%$ to vocational college or university, while the corresponding figures for students with high educated parents are $23 \%$ to university and $45 \%$ to vocational college or university. Again, including vocational college as a higher-education track clearly increases the probability of all groups participating in higher education. The percentage of students with low and medium educated parents going to vocational college or to university is three times as large as the percentage going exclusively to university. For students with high educated parents, this percentage is two times as large. The counterfactual proportions suggest some higher influence of secondary effects in the first two cohorts, while the relationship between primary and secondary effects becomes more balanced in the two younger cohorts.

\subsubsection{Primary and secondary effects at the age 17-18 transition}

The relative importance of secondary effects in the conditional models in Table 2.7 somewhat fluctuates, indicating neither a clear decrease nor increase over time. The proportion of total inequality explained by secondary effects is generally high at an overall average level of $94 \%$ at the transition from pre-university track (VWO) to university (WO) and at an overall average level of $81 \%$ at the transition from precollege track (HAVO) or pre-university track (VWO) to vocational college (HBO) or university (WO) (see also Figures A2.6 and A2.7 in the appendix). The relatively high importance of secondary effects at the transition from pre- 
university track to university consolidates the transition to university as an elite path. The conditional transition to vocational college or university generates lower proportions of secondary effects than the transition to only university, but it does not offer too much in terms of a distinctive decrease in IEO over time. The findings at this transition suggest that, to a large extent, the dominance of secondary effects results from the selection process at the first transition. ${ }^{23}$

Table 2.7: Relative importance (\%) of secondary effects at conditional transition at age 17 or 18: (1) from pre-university track (VWO) to university (WO) and (2) from precollege track (HAVO) or pre-university track (VWO) to vocational college (HBO) or university (WO)

\begin{tabular}{lcccccccc}
\hline & \multicolumn{2}{c}{1977} & \multicolumn{2}{c}{1982} & \multicolumn{2}{c}{1989} & \multicolumn{2}{c}{1993} \\
& $(1)$ & $(2)$ & $(1)$ & $(2)$ & $(1)$ & $(2)$ & $(1)$ & $(2)$ \\
\hline Medium-low & 90 & 79 & 93 & 87 & 91 & 71 & 94 & 84 \\
High-low & 93 & 79 & 96 & 88 & 91 & 78 & 95 & 80 \\
High-medium & 95 & 80 & 99 & 89 & 91 & 82 & 96 & 77 \\
Average & 93 & 79 & 96 & 88 & 91 & 77 & 95 & 80 \\
\hline
\end{tabular}

Source: CBS cohorts 1977, 1982, 1989, 1993; our calculations.

Table 2.8: Relative importance (\%) of secondary effects at unconditional transition at age 17 or 18: all students to (1) university (WO), and to (2) vocational college (HBO) or university (WO)

\begin{tabular}{lcccccccc}
\hline & \multicolumn{2}{c}{1977} & \multicolumn{2}{c}{1982} & \multicolumn{2}{c}{1989} & \multicolumn{2}{c}{1993} \\
& $(1)$ & $(2)$ & $(1)$ & $(2)$ & $(1)$ & $(2)$ & $(1)$ & $(2)$ \\
\hline Medium-low & 56 & 54 & 54 & 57 & 39 & 42 & 39 & 49 \\
High-low & 58 & 55 & 61 & 60 & 50 & 48 & 48 & 48 \\
High-medium & 59 & 56 & 66 & 62 & 56 & 52 & 54 & 46 \\
Average & 58 & 55 & 60 & 60 & 48 & 47 & 47 & 48 \\
\hline
\end{tabular}

Source: CBS cohorts 1977, 1982, 1989, 1993; own calculations.

Looking at the unconditional models in Table 2.8, we note that the relative importance of secondary effects is clearly lower than in Table 2.7 and on average also decreases somewhat over time. It suggests that, insofar as any change occurred, this change

\footnotetext{
${ }^{23}$ We performed a robustness check using performance tests at age 15 (see Table A2.6 in the appendix). There are some differences between the results using performance tests at age 15 and those using performance tests at age 12. Table A2.6 shows that when employing performance scores at age 15 the average relative importance of secondary effects at the transition to vocational college (HBO) or university (WO) is lower in the 1977 cohort (38\%) and almost the same in the 1981 cohort (47\%), compared to the analyses employing performance scores at age 12. In comparing particular background groups, we see that, when employing performance scores at age 15 , for both cohorts the relative importance of secondary effects is lowest between pupils of medium and low educated background, moderate between pupils of high and low educated background, and highest between pupils of high and medium educated background. While this trend is also found using test scores at age 12 in the 1989 cohort, it differs for the 1993 cohort.
} 
appears at the transition at age 12, rather than at the transition at age 17 or 18 . A comparison of these results with the relative importance of secondary effects at the first transition suggests that at the transition at age 17 or 18 there are no possibilities to repair previous decisions: later choices do not compensate for the choices made at the first transition. The proportions of secondary effects both at the transition to university and at the transition to vocational college or university are even higher than at the first transition at age 12, suggesting an ongoing selection in favor of pupils with high educated parents. Over time, the relationship between students with high and low, and high and medium educated parents shows that those of lower social background did catch up to some extent with those of higher social backgrounds but that secondary effects appear to remain more important than primary effects at these later transitions. The unconditional models suggest an average overall relative importance of secondary effects of 53\%.

\subsection{Conclusion}

This chapter has assessed the relative importance of primary and secondary effects at the transitions at age 12 (entry to secondary education) and age 17 or 18 (entry to higher education) in the Dutch educational system over time. Using panel data from four birth cohorts, we made an explicit distinction between the most prestigious and less prestigious routes in the higher tracks of secondary and higher education. In particular, we differentiate between the pre-university track (VWO) and the precollege track (HAVO) in secondary education and between university (WO) and vocational college (HBO) in higher education. Usually regarded as the less prestigious track in higher education, vocational college has gained in popularity among students over time. In particular, students from low and medium educated parents who complete a precollege track or a pre-university track are choosing to enter higher education through vocational college. Students from high educated parents, in contrast, are more likely to choose straight career paths from pre-university track to university.

Our results show that the relative importance of secondary effects at the first transition at age 12 has decreased over time and now amounts to an average of $38 \%$ of total IEO. While secondary effects lose some of their power at this transition, their importance clearly grows at later educational transitions. At the conditional transition to higher education at age 17 or 18, secondary effects gain a great deal in importance and almost exclusively explain the (conditional) transition to university (WO). While the proportions in the 
conditional model stay rather stable over time, the unconditional model at this transition shows a modest decrease in the relative importance of secondary effects, due to changes in the importance of secondary effects at the first transition. Although the Dutch educational system claims to be meritocratic in its selection into the different tracks of secondary education, we can observe that a large proportion of IEO is still related to differential preferences across social-background groups. At this point, almost $40 \%$ of the IEO differences are choice related.

The situation does not improve if we look at later transitions. At age 17 or 18 secondary effects have increased rather than decreased over the career, despite the Dutch educational system offering opportunities to repair mistakes by allowing moves between tracks. The decrease in secondary effects over time (from some $60 \%$ to some $48 \%$ ) is mainly due to changes at the first transition at age 12 .

These findings imply that policy measures by the Dutch government have mainly been successful at the first transition to secondary education by reducing choice related differences between social background groups, but they have largely failed at later transition stages. To provide an example, a 1993 reform aimed to restructure secondary education. The so-called Basisvorming was supposed to provide pupils of all secondary education tracks with a standardized basic curriculum in the first three years of secondary education and an opportunity to prove their abilities and preferences over a longer period before deciding on a final track. However, as shown in the evaluation by the Dijsselbloem parliamentary committee (see Borghans, van der Velden, Büchner, Coenen, and Meng, 2008), this reform did not lead to a considerable reduction in IEO. Pupils in our most recent birth cohort (the 1993 cohort) should have profited from this restructuring and subsequently experienced reduced inequality after entering secondary education. And yet the difference in IEO between this cohort and the previous cohort in our analysis is hardly detectable.

The issue is not exclusively related to secondary effects but also to the fact that primary effects contribute to IEO. Our findings suggest even increasing proportions of performance related differences at the first transition. In their cross-national comparison, that includes our results for the Netherlands, Jackson and Jonsson (2013) show that primary effects have a rather stable impact across countries, implying that "a floor level of IEO [exists] that secondary effects build on” (ibid:330). With regard to policy interventions it seems more difficult to tackle primary effects, as they are determined by genetic and early environmental features of the family background. However, there are examples of pre-school programs that prove early public interventions to be effective in reducing the school 
performance gap between children of different social backgrounds (e.g. Barnett, 2008; Heckman, 2006; Heckman, Pinto, and Savelyev, 2012). It is to be expected that such investments on disadvantaged children demand increasing costs, if they are applied nationwide and for a longer period. But positive returns have been traceable both for the short run and the long run (Barnett, 2008).

Nevertheless, future policy interventions should also focus on reducing the size of secondary effects at later educational transitions. The size of secondary effects, and thus the size of IEO, are to a considerable extent dependent on the structural features of the education system and probably can be easier manipulated by policy actions than primary effects (Jackson and Jonsson, 2013). Possible interventions could target, for example, subsidies for talented, disadvantaged students, to enter higher and putative riskier educational tracks. Another strategy could pursue to "push and pull” (Jackson and Jonsson, 2013:331) talented, disadvantaged students entering higher educational levels; for example, by professional guidance throughout their educational career, and/or by active recruitment by the higher education institutions. Such strategies, whether targeting financial incentives or improving the individual's information about higher educational tracks and possible chances of success, aim to increase these students' propensity entering more prestigious education levels, and thus, converging their transition behavior more to that of better-off students. 
3 Social background's effect on educational attainment: Does method matter? 


\subsection{Introduction}

Research has found a strong relationship between the parent's social status and the offspring’s educational achievement (e.g., Bourdieu, 1973; Mare, 1981; Shavit and Blossfeld, 1993). ${ }^{1}$ A child's social background directly impacts available educational choice and attainment but also indirectly influences choices and attainment by affecting pre-school cognitive-skills development as well as subsequent school performance. Boudon (1974) described this relationship as primary (indirect) and secondary (direct) effects of social stratification, as they explain inequality of educational opportunity (IEO) across social strata. Thus, throughout the educational career, primary effects reflect differences in performance at a given point, and secondary effects reflect differences in choices made at different branches or transition points. In Boudon's model, primary effects occur at the first transition, for example, the transition from primary to secondary education but not at later transitions. At the first transition, primary effects clearly outweigh secondary ones. At later transitions, however, secondary effects continue exerting influence. The cumulative effect of these differential choices may well outweigh performance's differential effects if enough branches occurred, necessitating numerous such choices.

Mare (1979, 1980, 1981) has taken this notion a step further and developed a model that considers sequential transitions from compulsory until tertiary education, conditional on choices made at previous stages of the educational career. At each stage, the probability of entering or leaving the next higher educational level is dependent on the social background. Moreover, Mare (1981) adds an important aspect by explicitly distinguishing between two possibly offsetting - trends in IEO. One concerns changes in transition probabilities: IEO has declined, as a result of educational expansion, leading to higher transition rates of all social classes but more rapidly for the lower than for the higher classes (due to ceiling effects). The other trend is social background's effect on transition probabilities, which may decrease or increase over time. Failing to properly distinguish this tendency in the statistical model may obscure these two different trends. Mare demonstrates the logistic response model adequately captures the "true" socioeconomic effect, while the linear model combines the two effects, noting: "No model or measure is the best or correct way to represent [IEO]” (Mare 1981:83), but the two do imply distinct conceptions of inequality. The linear model focuses more on absolute changes in IEO, for example, by showing that the lower classes' transition

\footnotetext{
${ }^{1}$ This chapter is based on joint work with Rolf van der Velden and Maarten Wolbers.
} 
probabilities have greatly expanded. The logistic model concentrates on relative chances, showing, for example, that for the few students who have not made this transition, probability has become more dependent on social background.

Both Boudon's and Mare’s publications have been very influential in IEO research. Several studies have examined IEO changes over time (e.g., De Graaf and Ganzeboom, 1993; Jackson et al., 2007; Raftery and Hout, 1993; Schindler and Reimer, 2010) or IEO differences across countries (Blossfeld and Shavit 1993). With few exceptions, however, most work has concentrated on one transition only. For example, Erikson et al. (2005), Jackson et al. (2007), Kloosterman et al. (2009), and Schindler and Reimer (2010) applied a counterfactual model to analyze the relationship among social background, school performance, and educational attainment at one specific transition in the educational career. While Erikson and Jonsson (1996) do estimate the transition probability for different transitions (the transition to higher secondary and university education) in Sweden, they do not explicitly examine these tracks' completion rates. Such a focus, however, limits analysis on Boudon's claim that secondary effects resume their importance at later transitions, and whether or not primary and secondary effects' relative importance varies at different transition and completion stages during the educational career.

Moreover, almost all authors use only one statistical method to assess primary and secondary effects' relative contributions and - even more important - do not clearly outline their model's assumptions and the implications it has for measuring either primary and secondary effects' relative weight or IEO.

Therefore, in this paper, we aim to shed more light on two issues. First, we decompose both primary and secondary effects to estimate their relative importance at an educational career's various stages in the Netherlands over a 25-year period. We explicitly distinguish between four stages of said educational career: transitioning from primary to secondary education tracks, completing the secondary track, transitioning from secondary to postsecondary education tracks, and completing the postsecondary track. As far as we know, with the exception of Mare's, no study has systematically explored these stages' completion. Previous studies on the second transition ignore the fact that this results from two different processes: successfully completing a previous track and choosing a postsecondary track. We cannot take for granted that merely choosing a secondary educational academic track will automatically lead to its successful completion. Moreover, the factors affecting completing a track may be quite different from those affecting its choice, for the former can be a function 
of performance and thus related to primary effects, while the latter can be a function of secondary effects.

Second, we systematically apply and compare different statistical methods. Two of these methods are commonly used: the counterfactual model and the linear regression model. We explore these models' basic assumptions and relate them to different IEO concepts. Next to these two models, we apply structural equation modeling, which offers as an attractive feature in that it explicitly separates structural relations between variables from the measurement model. This is important, because the way previous studies have estimated secondary effects' relative weight depends on a precise measurement of both performance and social background. Any noise in these measures will underestimate social background's total effect and bias the relative weight of secondary effects. Such biases can lead to the wrong policy implications. If policy makers want to reduce IEO, they need to know how strong the effect is and whether social class differentials are primarily performance-related or choice-related.

We use panel data from three large school cohort surveys from Statistics Netherlands that provide information about students' social background and cognitive skills at the beginning of secondary education and, on an annual basis, their school progress until the end of formal education. The data allow for a 25-year-long observation period, covering 1977 through 2004. Based on the theoretical approach of Boudon and Mare's sequential transition model, we will decompose primary and secondary effects to estimate their relative importance at different stages in the educational career. The first stage studies the choice of an academic or vocational track when entering secondary education. The second stage examines the successful completion of a secondary educational track, given that a track has been attended. The third stage considers the transition to a postsecondary education academic or vocational track, given that secondary education has been completed. The fourth stage focuses on the completion of a track in postsecondary education, given that a track has been attended. Furthermore, we will estimate an unconditional model in which we look at social background's total effect on the highest educational level attained. We will systematically compare the results for the three different analytical models.

The related research questions are:

1. To what extent does the relative importance of primary and secondary effects change in the Netherlands during the observation period, 1977-2004?

2. To what extent does the relative importance of primary and secondary effects change over different stages in the educational career? 
3. To what extent are the results dependent on specific methods that were used: counterfactual model, linear regression model, and structural equation model?

Our findings show secondary effects' diminishing impact at the first transition over time with a $4 \%$ to $10 \%$ decline - depending on the method - but a rather stable and, in some cases, expanding impact of secondary effects at the education career's later stages. As a result, the cumulative share of choice-related differences in social background's effect on educational attainment remains stable over time, at least if one examines the last two cohorts. The declining trend of secondary effects at the first transition is consistent with modernization theory assumptions, which suggest an overall higher demand for education. The linear regression models, however, show that in the last stage, at the successful completion of postsecondary education, the relative importance of secondary effects show movement in the opposite direction that rises over time. This suggests that preferences and expectations of aspiring higher education at the postsecondary level remain increasingly associated with social background.

Our findings indicate that applying different methods affects primary and secondary effects' relative magnitude: namely, method matters. Secondary effects are assessed to be some $43 \%$ of social background's total effect at the first transition and some $55 \%$ at the final obtained degree if one uses linear regression models or counterfactual models. However, if one uses structural equation modeling that allows taking into account measurement error in the performance and the social background measures, the secondary effects' relative importance declines to some $32 \%$ at the first transition and to some $47 \%$ at the final grade completed. In other words, the most commonly used methods (the counterfactual model and the linear regression model) overestimate choice-related class differentials' relative weight by some $8 \%$ to $11 \%$. Nevertheless, even the structural equation model illustrates that preferences and expectations of aspiring higher educational levels remain strongly associated with social background.

The remainder of this paper is organized as follows. Section 3.2 discusses the theoretical background and formulates hypotheses with regard to the empirical analysis. Section 3.3 introduces the three statistical methods that are used to test the hypotheses and discusses their assumptions and their pros and cons. Section 3.4 describes the data and operationalization of the variables. Section 3.5 presents the empirical findings in detail. The last section summarizes the main results and draws conclusions. 


\subsection{Theoretical background}

In this section, first we provide background information about the theoretical concept and discuss its related advantages and issues. Next, we introduce our hypotheses for the empirical analysis.

Decomposing social background into primary and secondary effects helps determine the ways the two effects have an impact on educational choices and attainment. According to Boudon (1974), primary effects reflect children's cognitive and non-cognitive abilities, inherent with family background. Secondary effects reflect educational preferences that are chosen in reference to a certain social environment and previous cognitive performance.

Recent studies on child development show that abilities are highly correlated with both the family's genetic background and the child's environmental conditions. Differences in ability show up at early ages and manifest differently across socioeconomic groups (e.g., Carneiro and Heckman, 2003; Gluckman and Hanson, 2005; Heckman, 2007). Bettereducated parents tend to provide more intellectual stimulation, which results in more ambitious educational choices and successful achievement of their children (Erikson and Goldthorpe, 2002; Shavit and Blossfeld, 1993).

Choosing a specific type of educational path, however, is not only dependent on endowments but also on preferences, as well as on constraints imposed by a family's cultural and economic resources. Boudon defines these (class) differences as secondary effects of social stratification; that is, how individuals evaluate the costs and benefits of pursuing higher education, including financial and social costs and expectations of educational success. Assumptions of cultural capital theory (Bourdieu, 1973; Bourdieu and Passeron, 1977), cultural reproduction theory (e.g., Collins, 1971), and rational action theory (e.g., Breen and Goldthorpe, 1997) all point in the same direction. Higher-status families choose more demanding tracks for their children, since they do not want to risk social demotion: They want to provide their children with enough opportunities so that their offspring might be able to maintain the socioeconomic status they have attained themselves. Cultural reproduction theory argues educational certificates thereby serve to both exclude members of lower-status groups from desirable positions in the occupational structure and keep higher education and higher-level job positions as privileges of dominant social groups (cf. Blossfeld and Shavit, 1993:217). Rational action theory suggests the effort higher-status families make is less costly than the same would be for lower-status families. Lower-status families, in contrast, must make more burdensome sacrifices and therefore must have relatively stronger ambitions 
when they send their children to school for longer periods (Gambetta, 1987). Socioeconomic differences thus are driven by rational decisions of rational actors at various transitions in the educational career (Breen and Goldthorpe, 1997).

With respect to changes over time, modernization theorists argue that, due to industrialized societies' requirements of an educated labor force, the educational qualifications' importance rises in the labor market where educational expansion serves as a tool that responds to these requirements. The theory goes that with increasing modernization, socioeconomic differences should diminish and educational selection will become more meritocratic (Parsons, 1970; Treiman, 1970). Indeed, ever since enhanced expansion and access to the educational system in the 1960s, educational participation of less-privileged groups has improved throughout all Western societies. In countries like the Netherlands, the educational system's restructuring was part of the attempt to mitigate ascriptive criteria's influences in favor of more meritocratic structures. This was conducted by introducing nationwide compulsory skills tests, primary school teachers' school-type recommendations, and orientation classes at the transition to secondary education (Dronkers, 1993:262f.). Several studies about the Netherlands (e.g., Bosma, Dronkers, and Hagenaars, 1991; De Jong, 1982; De Jong et al., 1982; Dronkers and Bosma, 1990) pointed out these measures reduced social background's effect on educational attainment at the transition from primary to secondary education.

Within our conceptual framework, we thus have two different time perspectives. One is the perspective within a school cohort looking at an individual's school career and analyzing to what extent social background's effect changes at various school transitions. The other perspective is between school cohorts where we compare social background's effects on particular school transitions over time.

Regarding our perspective within a school cohort, we hypothesize primary effects are stronger at the first transition (H1), while secondary effects are stronger at the second transition (H2). Current theory is not specific enough to allow a hypothesis on the relative weight of primary versus secondary effects on the completion of the secondary and postsecondary track.

Regarding the perspective of changes between cohorts, we follow modernization theory, which claims the educational expansion assigned greater weight to meritocratic selection with increasing graduation rates in higher secondary education. We therefore hypothesize secondary effects have diminished over our observation period for the transition 
to secondary as well as to postsecondary education (H3). We also assume completing these tracks increasingly will be determined by primary effects (H4).

\subsection{Analytical strategy}

In this section, we introduce the statistical techniques and their implications for the theoretical model. The statistical techniques under use are a counterfactual model, a linear regression (ordinary least squares or OLS) model, and a structural equation model using LISREL. The first two models have often been used in stratification research but have seldom been directly compared, while the third model is less often used but in our view, offers important advantages over the other two methods.

All three estimation methods as well as the way they are modeled come with different assumptions about the underlying concepts and mechanisms. The main differences relate to the concept of IEO, the concept of social background and educational attainment, the use of conditional or unconditional models, and the treatment of measurement error.

\section{Absolute or relative IEO?}

As indicated by Mare (1981), the main difference between the logistic response model and the linear model is how they view IEO. The logistic response model (and thus the counterfactual model) concentrates on socioeconomic background effect, given a certain overall transition probability, while the linear model (and the same applies for the structural equation model in our analysis) examines the combined effect of these two on IEO. The difference can be expressed that in terms of absolute IEO, transition probabilities for lower classes have greatly expanded, while for the few students who have not made this transition, the probability has become more dependent on social background.

\section{Continuous or categorical concepts of social background and educational attainment?}

The counterfactual model uses a class approach toward social stratification where the main dividing lines are situated between a limited number of rather homogeneous classes, with respect to both social background and educational attainment. This is congruent with a view on the Dutch educational system as a construct supporting (self-reproducing) social class patterns. 
The OLS and LISREL models basically view social background and educational attainment as continuous variables, rather than categorical variables, which is more in line with a social status approach. It is congruent with a view on social stratification in which the division of social positions can be depicted as a ladder and where acquiring resources, such as education and social capital, enables one to ascend that ladder.

\section{Conditional or unconditional models?}

In both the counterfactual model as well as the OLS model, we will use conditional models to examine stratification effects at each stage of the educational career. The conditional model implies at each stage, the group under analysis becomes smaller, as we look at only those who passed a certain transition. This is in accord with a view on education that regards the selection regime as a form of contest mobility (Turner, 1960), meaning a limited number of transitions exist and at each transition, a selection is made between a higher and a lower track. This selection takes place based on performance and social background. Once a person is referred to a lower track, (s)he cannot return to a higher track. We will also estimate unconditional models in all three analyses by examining the highest degree obtained for the whole group. This is more consistent with a view on the education system as a permeable strata system providing alternative pathways to higher education throughout the school career. $^{2}$

\section{The treatment of measurement error}

In the OLS and counterfactual models, primary effects are defined by the effect of school grades or performance tests on transitions to higher education. Secondary effects are then defined as the remaining effect of social background, after controlling for socioeconomic differences in performance. School grades and performance tests, however, measure the "true" skills with measurement error. The more noise they produce, the lower the estimated primary effects will be and hence the higher the secondary effects will be. The same holds for measuring social background. Usually estimated by the parents' educational level or occupational status, the social background's overall effect on educational attainment is likely

\footnotetext{
${ }^{2}$ Breen and Jonsson (2000), who analyzed the Swedish educational system, which is comparable with that of the Netherlands, suggest different pathways that can be chosen in a parallel educational track system affect the probability of making subsequent educational transitions to a great extent (p. 754). In our analysis, we will take into account the different educational tracks in the Dutch school system and also systematically distinguish between vocational and academic school tracks at the secondary and postsecondary levels.
} 
to be underestimated in linear and counterfactual models. ${ }^{3}$ We assume that secondary effects in the OLS and counterfactual models are generally overestimated. The structural equation model explicitly accounts for this measurement error and allows for a more unbiased estimate of the secondary effects of social stratification.

\subsubsection{Counterfactual model}

The counterfactual model has recently been introduced by Erikson (2007) and Jackson et al. (2007) to estimate factual and counterfactual transition probabilities to higher secondary education, conditional on average school performances across social classes and over time. The model is based on the logistic response model and has as an attractive feature directly comparing what would happen to the transition probability of a certain status group if they would have the cognitive abilities or the preferences of a different group. The counterfactual method has successfully been applied in several studies to disentangle preferences and endowments and to measure social class differences in educational attainment (Erikson, 2007; Jackson et al., 2007; Kloosterman et al., 2009; Schindler and Reimer, 2010). We adopt the method for the Netherlands to measure the average scholastic performance and transition and completion probabilities to academic tracks in secondary and postsecondary education for children from three different social class backgrounds. We group our social background variable into lower-educated parents (6-10 years of schooling), medium-educated parents (14 years of schooling), and higher-educated parents (17-19 years of schooling). Primary effects of social stratification will be estimated by the mean and the standard deviation of the school performance scores, separately for each social class. The school performance scores are assumed to be normally distributed. For the transition probability to higher (post-) secondary education, we estimate binary logistic regressions measuring the effect of scholastic performance per social class.

To measure the relative importance of primary and secondary effects, we apply the method of numerical integration (Jackson et al., 2007). For each social class, we calculate the proportion of students' transition probability to academic tracks in secondary and postsecondary education, given their (normally distributed) performance scores per class. The integral is written as

\footnotetext{
${ }^{3}$ This issue of measurement error is seldom explicitly discussed. Jackson et al. (2007), for example, focus on the issue of anticipatory decisions (the effect of preferences on the test itself), but leave aside the-probably more important-issue of measurement error in the test.
} 


$$
\int_{-4}^{+4}\left(\frac{1}{\sigma \sqrt{2 \pi}} e^{-(x-\mu)^{2} / 2 \sigma^{2}}\right)\left(\frac{e^{(a+b x)}}{1+e^{(a+b x)}}\right) d x
$$

where $\mu$ indicates the mean of the performance scores and $\sigma$ the standard deviation; $a$ is the constant and $b$ the logit coefficient that indicates the performance of a student of a certain social class. The term within the first parenthesis refers to the normalized distribution of the average performance score per social class, while the term in the second parenthesis indicates the transition probabilities obtained by the logistic regression. The advantage of numerical integration is that the two components can be separately calculated for any class transition and therefore enables to carry out counterfactual models. That means that, ceteris paribus, combinations of class-specific performance distributions with performance-specific transition propensities of any other social class are possible (cf. Jackson et al., 2007:218f). To give an example, we can combine the distribution of performance scores of children from highereducated parents with the performance-specific transition probability of children from lowereducated parents.

\subsubsection{Linear regression model}

Using a linear regression model, we assume a linear relation between social background, test scores, and the school track choice or completion. We decompose the effects by estimating the total effect of parental background and the secondary effect of social background, controlling for cognitive skills. ${ }^{4}$ The equation for the total effects of social background can be written as

$$
Y_{1 i j}=\alpha_{0 i j}+\beta_{1} P E D_{i}+\varepsilon_{i j}
$$

where $i$ denotes the individual and $j$ the transition to or completion of a certain school track. $Y_{1 i j}$ is the probability for the transition to, or completion of a certain (post-) secondary educational track, respectively. $\alpha_{0 i j}$ denotes the constant and $\beta_{1 j} P E D_{i}$ the coefficient for the effect of parents' education at a certain stage (transition/completion) within the school career, while $\varepsilon_{i j}$ denotes the error term. The estimation of secondary effects of social background is done in two variants, an unconditional estimation of the cumulative secondary effects, and an

\footnotetext{
${ }^{4}$ See also Buis (2010a) for the definition of direct, indirect, and total effects of social background.
} 
estimation of direct secondary effects that are conditional on the previous tracks that an individual has chosen. Regarding the cumulative secondary effects, we write

$$
Y_{2 i j}=\alpha_{0 i j}+\beta_{1} P E D_{i}+\beta_{2} P E R F_{i}+\varepsilon_{i j}
$$

where $\beta_{2 j} P E R F_{i}$ denotes performance scores of a student obtained at the end of primary education. Regarding direct secondary effects of social background we additionally control for previous transitions within the educational career, i.e.

$$
Y_{3 i j}=\alpha_{0 i j}+\beta_{1} P E D_{i}+\beta_{2} P E R F_{i}+\beta_{3} \operatorname{STRACK}_{i j-1}+\varepsilon_{i j}
$$

where STRACK denotes the school track at time t-1. By subtracting the estimated coefficients of social background either of equation (3.2) or (3.3) from the estimated coefficient of social background of equation (3.1), we obtain an estimate of the secondary effects.

\subsubsection{Structural equation model}

As indicated above, both the counterfactual and the OLS model simply assess the secondary effects as the effects of social background after controlling for cognitive skills. This way of decomposing the total social background effect into primary and secondary effects is vulnerable to measurement error, especially of cognitive skills. We thus apply a structural equation model with latent variables that allows to capture measurement error and to estimate the structural relations between the latent variables more accurately. In a first model we treat cognitive skills as a latent variable that is defined by the CITO math and language scores measured at age 12 (for a detailed explanation, see data section below). The lambda parameter of the math score is fixed to one, while the lambda parameter of the language score is kept free. The latent variable cognitive skills is our main interest, since estimating performance scores from a relatively short test is most vulnerable to measurement error. The latent variable social background is defined by the average of parents' education, first choice secondary education is defined by the first school track students enter at age 12, and final educational degree is defined by the overall years of schooling observed per student. In this first model, all lambda parameters for the latent variables, except cognitive skills, are fixed to one and the measurement error is fixed to zero. 
In a second model we also take measurement error in the latent social backgroundvariable into account, as the average of parents' education is also sensitive to measurement error and thus likely to underestimate the total effect of social background on educational attainment. We re-define the latent variable social background by single measures for father's and mother's education, ${ }^{5}$ fixing the lambda parameter of father's education to one, and keeping the lambda parameter of mother's education free. This new latent variable replaces the former latent variable for social background in the first model.

\subsection{Data}

The analysis uses panel data of three school cohorts provided by Statistics Netherlands (Centraal Bureau voor de Statistiek, CBS). Each cohort is a national representative sample of students at age 12 who enter secondary education. The three cohorts have the same design. Data has been collected in $1977(\mathrm{~N}=37,280), 1989(\mathrm{~N}=19,524)$ and $1993(\mathrm{~N}=20,331)$, respectively. ${ }^{6}$ At the start of the cohort, at age 12, information is gathered on performance in language, math and some other personal characteristics for all students in the class as well as a large number of background characteristics through interviewing the parents. Annual information about subsequent school track choices and completion is collected until the end of full-time education for almost all students in the sample. ${ }^{7}$ Each cohort is analyzed separately on the basis of comparable information available in all cohorts.

\subsubsection{Dependent variables}

As mentioned above, we want to investigate the influence of primary and secondary effects at four different stages during the educational career: at the transition from elementary school to secondary education (t1), at the completion of secondary education, given secondary education has been entered (t2), at the transition to postsecondary education, given secondary education has been completed $(\mathrm{t} 3)^{8}$, and at the completion of postsecondary education, given

\footnotetext{
${ }^{5}$ This model could still improve, if we would add parents' occupational status to the latent variable social background. Unfortunately, this information was not available in the data.

${ }^{6}$ For this analysis we leave out the cohort that started in 1982. The sample design and the parental questionnaire of that cohort differ too much from the three other cohorts to make them really comparable, especially as we will use different statistical methods.

${ }^{7}$ If a pupil drops out of the survey before he or she has finished a (final) grade, we use the last information available about the educational choices and attainment.

${ }^{8}$ A student has completed the secondary education level successfully, if he or she passed a final exam and/or proceeded to postsecondary education. If a student is observed at a certain track at postsecondary education level but leaves this track without a passed exam, he/she will be allocated to the exam passed before at
} 
postsecondary education has been attended (t4). ${ }^{9}$ The dependent variables include the choice or completion of school tracks in secondary and postsecondary education levels, respectively. In addition, we calculate an unconditional model of the highest degree that a student obtained. ${ }^{10}$

For the linear regression and structural equation model, we will use a detailed measure of these tracks, distinguishing all possible tracks in the Dutch school system. These tracks are coded into a specific years of schooling scale (the so-called 'years of schooling ladder'; cf. Van der Velden, 1991), which measures all educational positions as a function of how many years it will take to reach the top of the educational system, i.e. completing university education. The choice of tracks in secondary education is assigned the following scores: VWO (9), HAVO (8), MAVO (7), VBO (6), and IBO (5). Students who start in orientation classes of mixed school forms are assigned to one of these tracks as soon as they change to a single school type. The completion of these tracks is assigned the scores: VWO (15), HAVO (13), MAVO (11), VBO (10), and IBO (9). In postsecondary education the following scores are assigned to the choice of a particular school type: WO (15), HBO (13), 3 or 4 years MBO (11), and 1 year or 2 years MBO (10). Completing the tracks in postsecondary education is scored as: WO (19), HBO (17), 3 or 4 years MBO (14), and 1 year or 2 years MBO (12). For the counterfactual model we simply distinguish between academic tracks and vocational tracks in secondary and postsecondary education. We combine HAVO and VWO as the academic tracks in secondary education and HBO and WO as the academic tracks in postsecondary education. All other tracks are labeled as vocational tracks.

\subsubsection{Independent variables}

Social background. As a measure for social background, we take the average of the highest educational level attained by the parents, again coded in the 'years of schooling' scale. ${ }^{11}$ We

\footnotetext{
secondary education level. If no exam was passed at all or the information is missing, this student will not be taken into account, with regard to the unconditional model and the conditional model on completing postsecondary education.

${ }^{9}$ For cohort 1993 information is collected until school year 2003/2004, when students are aged 22. Some respondents may not have finished education at higher levels at that time and thus cannot be considered at stage t4.

${ }^{10}$ Based on our school continuation model we only consider students who stay in education. Persons who drop out from school after secondary education are not taken up in the subsequent analysis. We assume that this will have a downward bias on the estimation of the secondary effects.

${ }^{11}$ Most studies on primary and secondary effects (e.g., Erikson et al. 2005; Jackson et al. 2007; Schindler and Reimer 2010) use the occupational status of the parents, but this information is not available in our data. However, De Graaf and Ganzeboom (1993) show for the Netherlands that the educational attainment of the
} 
distinguish parental education into primary education (6 years), lower secondary education (MAVO or VBO: 10 years), higher secondary education (VWO, HAVO or MBO: 14 years), lower tertiary education (HBO: 17 years) and higher tertiary education (WO: 19 years). Across the cohorts, the share of parents with only primary and lower secondary education decreased, while the shares of parents with higher secondary education or tertiary education increased (not illustrated).

Cognitive skills/scholastic performance. As an indicator for cognitive skills, we use test scores based on standardized compulsory tests in mathematics and language, which are provided by the Dutch Central Institute for Test Development, the so-called CITO tests. Usually conducted at the end of primary education, the CITO tests estimate the state of knowledge, development and capability level of each student, before they enter secondary education. The tests provided in the data are conducted about half a year later and differ in the length and the scaling format from the regular CITO tests. They are constructed as repeated tests exclusively for the survey. Hustinx et al. (2005) found that the test results in the surveys are comparable with those of the original CITO tests and serve as good proxies. Anticipatory decisions in terms of affecting the performance level in successive examinations are not an issue here, since decisions already have been made on the basis of the regular CITO tests at the end of primary education. ${ }^{12}$ The tests were measured somewhat differently over the cohorts; therefore we normalized the scores on a scale from 1 to $100 .{ }^{13}$ Correlations between test scores and social background are between 0.32 (cohort 1977) and 0.37 (cohorts 1989 and 1993).

\subsection{Results}

For illustration purpose and an initial descriptive view on primary and secondary effects, Figures 3.1 and 3.2 show the distributions of scholastic performance and the logistic curves for making the transition to academic tracks in (post-) secondary education for cohort $1993 .{ }^{14}$

\footnotetext{
father is a better indicator in terms of predicting educational success of the offspring and is more resistant to changes over time, than the father's occupational status.

${ }^{12}$ For more information about anticipatory decisions, see Erikson et al. (2005), and Jackson et al. (2007).

${ }^{13}$ Note that we standardize the test scores to make the results comparable across cohorts. In a pooled analysis however, this would be an issue, as the 'wrong' standardization can have peculiar effects on other parameters.

${ }^{14}$ Figures A3.1 and A3.2 for cohort 1977 and figures A3.3 and A3.4 for cohort 1989 are displayed in the appendix.
} 
Figure 3.1: Academic performance and transition probabilities to academic tracks in secondary education, school cohort 1993

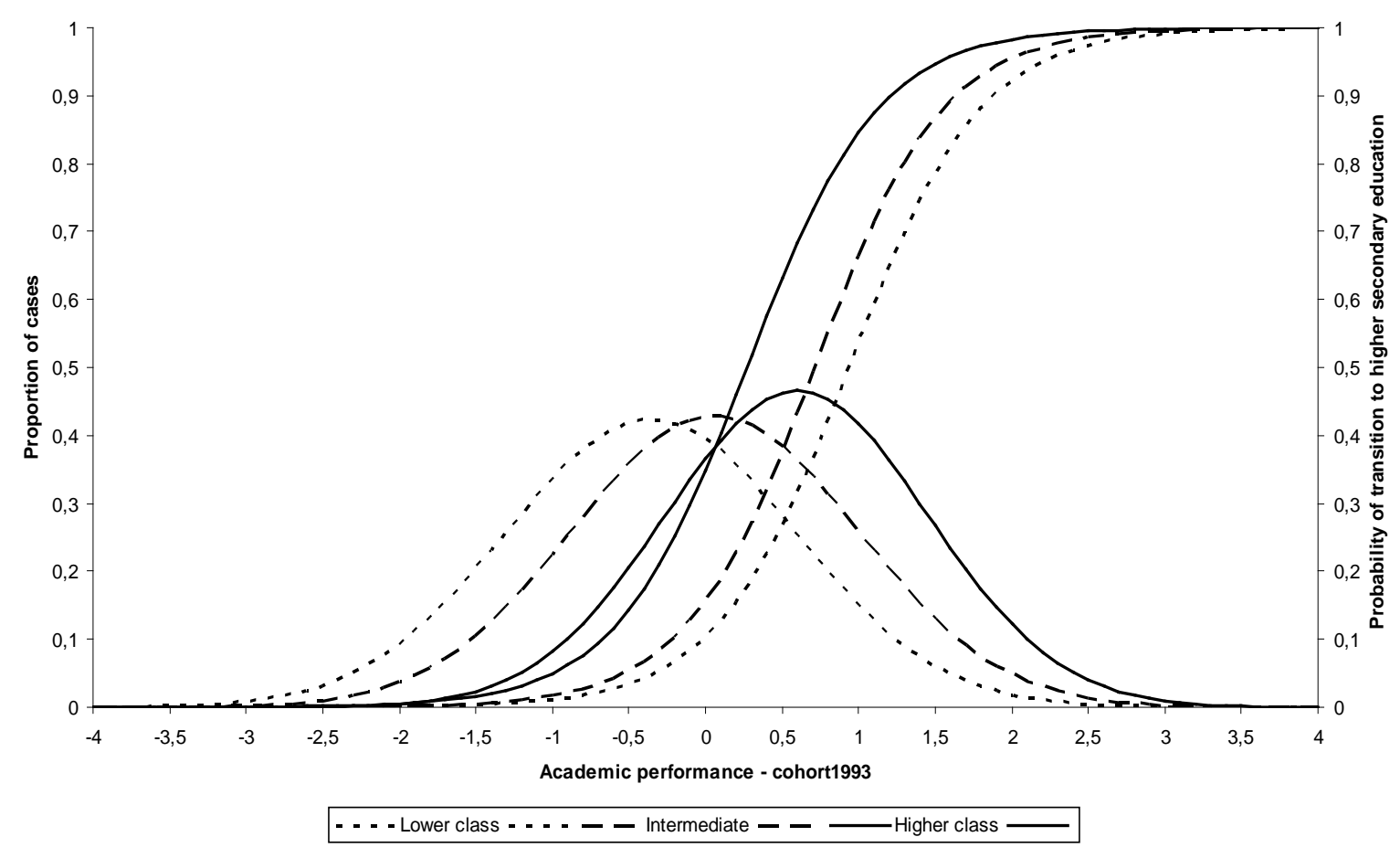

Figure 3.2: Academic performance and transition to academic tracks in postsecondary education, school cohort 1993

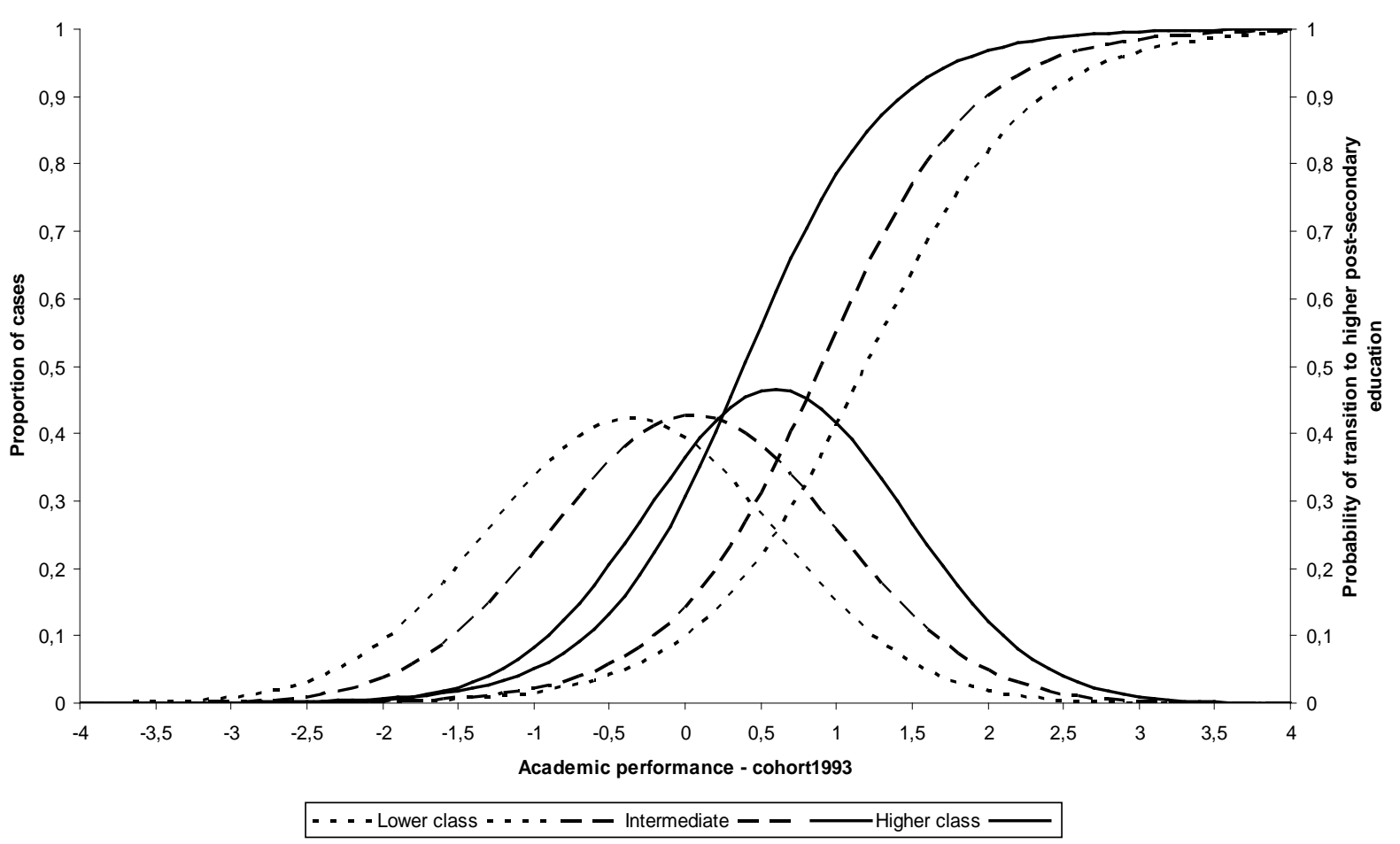


The figures show the performance distribution of students from higher-educated parents is always found situated far more to the right; that is, this group on average performs higher on performance tests than do the other two groups. Students from lower-educated parents on average perform the lowest of all groups, while the children from medium-educated parents fall in-between. These differences in primary effects are consistent over the whole observation period. Moreover, the distance between the socioeconomic groups has widened over time: The difference in the average performance scores between children from higherand medium-educated parents has increased from cohort 1977 to the youngest cohort. A similar picture can be seen when comparing children from higher- and lower-educated parents. The difference in average performance scores between children from medium- and lower-educated parents is less evident, but the results indicate primary effects increase over time.

With regard to the logistic curves over time, we observe several features about social class-specific transition propensities: Given a certain performance level, students from higher- educated parents are most likely to continue in academic tracks in secondary and postsecondary education over all cohorts, followed at some distance by children from medium- and lower- educated parents. Although children from higher-educated parents perform better on average, their transition propensity-curve also is located far more to the left, which signals that secondary effects already work at the performance distribution's lower end. The average academic performance distribution of children from higher-educated parents remains at a fairly stable level over time, while children from lower- and mediumeducated parents fared worse over time.

\subsubsection{Primary and secondary effects' relative importance using counterfactual models}

Table 3.1a shows the combinations of class-specific performance distributions with performance-specific transition propensities for the transition to academic tracks in secondary education. Table 3.1b shows the actual and counterfactual combinations for completing an academic track in secondary education, given that an academic track has been chosen. Table 3.1c continues with the combinations for the transition to academic tracks in postsecondary education, given that an academic track has been completed, and Table 3.1d reports the combinations for completing an academic track in postsecondary education, given that an 
academic track has been chosen. Table 3.1e presents the combinations for the unconditional model of obtaining an academic degree in postsecondary education.

The tables' rows refer to each social class's performance distribution and the columns to each social class's performance-specific transition probability. The diagonal from top left to down-right in each cohort reports each social class's observed, actual transitions. For example, in Table 3.1a, 14.2\% of the children from lower-educated parents of cohort 1993 advanced to an academic track in secondary education, whereas $61.6 \%$ of the children from higher-educated parents did so, given the average academic performance of their social class. Now, suppose that children from lower-educated parents had the same average performance as children from higher-educated parents but kept their own group's performance-specific transition propensities. In that case, the proportion of children who would proceed to higher secondary education would rise from $14.2 \%$ to $38.4 \%$ (down-left corner). Conversely, if they kept their performance level but had the same transition propensity as children from highereducated parents, the proportion would rise from $14.2 \%$ to $30.0 \%$ (upper-right corner).

Table 3.1a: Estimated probabilities of the transition to higher secondary education for actual and counterfactual transition rates

\begin{tabular}{lccccccccc}
\hline & \multicolumn{3}{c}{1977} & & \multicolumn{3}{c}{1989} & & \multicolumn{3}{c}{1993} \\
Parents' education & low & medium & high & low & medium & high & low & medium & high \\
\hline Low & 0.180 & 0.232 & 0.348 & 0.131 & 0.172 & 0.269 & 0.142 & 0.184 & 0.300 \\
Medium & 0.260 & 0.324 & 0.456 & 0.207 & 0.264 & 0.389 & 0.232 & 0.291 & 0.431 \\
High & 0.384 & 0.460 & 0.603 & 0.337 & 0.412 & 0.559 & 0.384 & 0.460 & 0.616 \\
\hline
\end{tabular}

Note: The diagonal cells with italic numbers show the factual transition rates, while the off-diagonal cells show the counterfactual transition rates.

Table 3.1b: Estimated probabilities of completing academic tracks in secondary education for actual and counterfactual transition rates

\begin{tabular}{lccccccccc}
\hline & \multicolumn{3}{c}{1977} & \multicolumn{3}{c}{1989} & & \multicolumn{2}{c}{1993} \\
Parents' education & low & medium & high & low & medium & high & low & medium & high \\
\hline Low & 0.264 & 0.343 & 0.498 & 0.165 & 0.207 & 0.341 & 0.159 & 0.215 & 0.350 \\
Medium & 0.348 & 0.437 & 0.602 & 0.247 & 0.303 & 0.466 & 0.250 & 0.323 & 0.481 \\
High & 0.387 & 0.564 & 0.728 & 0.376 & 0.447 & 0.629 & 0.399 & 0.488 & 0.658 \\
\hline
\end{tabular}

Note: The diagonal cells with italic numbers show the factual transition rates, while the off-diagonal cells show the counterfactual transition rates. 
Table 3.1c: Estimated probabilities of the transition to higher postsecondary education for actual and counterfactual transition rates

\begin{tabular}{lccccccccc}
\hline & \multicolumn{3}{c}{1977} & & \multicolumn{3}{c}{1989} & & \multicolumn{3}{c}{1993} \\
Parents' education & low & medium & high & low & medium & high & low & medium & high \\
\hline Low & 0.173 & 0.224 & 0.358 & 0.113 & 0.145 & 0.258 & 0.123 & 0.163 & 0.274 \\
Medium & 0.239 & 0.303 & 0.457 & 0.177 & 0.218 & 0.369 & 0.194 & 0.254 & 0.395 \\
High & 0.341 & 0.421 & 0.589 & 0.284 & 0.338 & 0.527 & 0.318 & 0.403 & 0.572 \\
\hline
\end{tabular}

Note: The diagonal cells with italic numbers show the factual transition rates, while the off-diagonal cells show the counterfactual transition rates.

Table 3.1d: Estimated probabilities of completing academic tracks in postsecondary education for actual and counterfactual transition rates

\begin{tabular}{lccccccccc}
\hline & \multicolumn{3}{c}{1977} & & \multicolumn{3}{c}{1989} & & \multicolumn{3}{c}{1993} \\
Parents' education & low & medium & high & low & medium & high & low & medium & high \\
\hline Low & 0.216 & 0.277 & 0.436 & 0.277 & 0.338 & 0.470 & 0.253 & 0.325 & 0.490 \\
Medium & 0.285 & 0.360 & 0.533 & 0.351 & 0.419 & 0.576 & 0.336 & 0.424 & 0.602 \\
High & 0.469 & 0.478 & 0.656 & 0.453 & 0.526 & 0.702 & 0.457 & 0.559 & 0.739 \\
\hline
\end{tabular}

Note: The diagonal cells with italic numbers show the factual transition rates, while the off-diagonal cells show the counterfactual transition rates.

Table 3.1e: Estimated probabilities of final academic tracks in postsecondary education: unconditional models for actual and counterfactual transition rates

\begin{tabular}{lccccccccc}
\hline & \multicolumn{3}{c}{1977} & & \multicolumn{3}{c}{1989} & & \multicolumn{3}{c}{1993} \\
Parents' education & low & medium & high & low & medium & high & low & medium & high \\
\hline Low & 0.128 & 0.181 & 0.311 & 0.168 & 0.230 & 0.376 & 0.183 & 0.260 & 0.440 \\
Medium & 0.177 & 0.241 & 0.384 & 0.231 & 0.304 & 0.461 & 0.256 & 0.348 & 0.534 \\
High & 0.253 & 0.333 & 0.483 & 0.327 & 0.410 & 0.570 & 0.368 & 0.474 & 0.654 \\
\hline
\end{tabular}

Note: The diagonal cells with italic numbers show the factual transition rates, while the off-diagonal cells show the counterfactual transition rates.

These counterfactual combinations give some insight concerning primary and secondary effects' relative importance, according to social background differences. They suggest children from lower social classes may catch up to some extent if they exhibit either the average performance or the transition propensity of the highest social class, but they never achieve or exceed this group’s proportions.

We further estimate secondary effects' relative importance in class differentials as proportions of log odds ratios; that is, actual log odds of a certain social class $i$ are compared with a counterfactual case where either the performance distribution or the transition probability of social class $i$ is replaced by that of social class $j$ (see Jackson et al. 2007:220f.). Tables 3.2a and 3.2b display secondary effects' relative importance at the transition to academic tracks in secondary and postsecondary education as a percent of log odds ratios for 
each cohort. Regarding entering the highest tracks at both transitions, Table 3.2a shows that secondary effects declined over time, from $45 \%$ to $41 \%$ at the first transition and from $51 \%$ to $45 \%$ at the second transition. Note that secondary effects' relative importance is greater for the transition to academic tracks in postsecondary education than for the transition to academic tracks in secondary education.

Table 3.2a: Relative importance of secondary effects, at the transition to academic tracks in secondary and postsecondary education (log odds ratios)

\begin{tabular}{|c|c|c|c|c|c|c|}
\hline & 1977 & 1989 & 1993 & 1977 & 1989 & 1993 \\
\hline & \multicolumn{3}{|c|}{ enter higher secondary education } & \multicolumn{3}{|c|}{ enter higher post-secondary education } \\
\hline highly educated / low educated & 0.46 & 0.43 & 0.42 & 0.53 & 0.48 & 0.47 \\
\hline highly educated / medium educated & 0.50 & 0.47 & 0.46 & 0.57 & 0.56 & 0.50 \\
\hline medium educated / low educated & 0.34 & 0.45 & 0.34 & 0.44 & 0.33 & 0.39 \\
\hline Average & 0.45 & 0.42 & 0.41 & 0.51 & 0.46 & 0.45 \\
\hline
\end{tabular}

Table 3.2b: Relative importance of secondary effects, at the completion of academic tracks in secondary and postsecondary education (log odds ratios)

\begin{tabular}{lcccccccccc}
\hline & $\begin{array}{c}1977 \\
\text { complete higher } \\
\text { secondary education }\end{array}$ & $\begin{array}{c}1977 \\
\text { complete higher post- } \\
\text { secondary education }\end{array}$ & $\begin{array}{c}1977 \\
\text { final post-secondary } \\
\text { education (unconditional) }\end{array}$ \\
\hline $\begin{array}{l}\text { highly educated / low educated } \\
\text { highly educated / medium }\end{array}$ & 0.55 & 0.48 & 0.46 & 0.57 & 0.58 & 0.57 & 0.55 & 0.53 & 0.55 \\
$\begin{array}{l}\text { educated } \\
\text { medium educated / low }\end{array}$ & 0.59 & 0.54 & 0.50 & 0.60 & 0.64 & 0.60 & 0.58 & 0.58 & 0.59 \\
educated & 0.49 & 0.36 & 0.39 & 0.48 & 0.46 & 0.48 & 0.51 & 0.49 & 0.51 \\
\begin{tabular}{l} 
Average \\
\hline
\end{tabular} & 0.54 & 0.46 & 0.45 & 0.55 & 0.56 & 0.55 & 0.55 & 0.53 & 0.55 \\
\hline
\end{tabular}

At each transition, the larger differences can be found between children from higherand medium-educated parents, and between children from higher- and lower-educated parents, compared with differences between children from medium- and lower-educated parents. Secondary effects' total contribution thereby increases a minor amount. For cohort 1993, it starts with $41 \%$ at the secondary education entry, rises to $45 \%$ at completing secondary education, stays at this level when entering postsecondary education, and increases again when completing this level (55\%). This shows that social background's effect is cumulative, and there is no possibility for remediation later in the career. The only exception is for cohort 1977, which shows a higher percentage at the point of completing the secondary track (54\%) than for choosing a postsecondary educational academic track (51\%). 
Secondary effects' overall importance, as shown in the unconditional model, is nearly the same as in the conditional model for completing postsecondary education. This illustrates there are no major alternative pathways in the education system that children from lowereducated parents can pursue to remediate or otherwise improve on their earlier educational choices.

With regard to our hypotheses, the counterfactual model's findings suggest the following: The first hypothesis can be confirmed, as primary effects are more important than secondary effects at the first transition to higher secondary education. The second hypothesis, however, is only confirmed for the oldest cohort, with secondary effects outweighing primary effects. For the two youngest cohorts, however, the primary effects remain dominant, even at this career stage. Furthermore, the decrease in secondary effects' relative importance over time, both at the first transition to secondary education and at the transition to postsecondary education, confirm the third hypothesis and accord with modernization theory, suggesting that these transitions become more meritocratic. In addition, when completing secondary education, we observe secondary effects decreasing, which partly confirms Hypothesis 4. However, at the last stage, completing postsecondary education, as well as in the unconditional model, secondary effects are relatively more important than primary effects, with a stable share over time. This finding refutes modernization theory's assumptions and is more in agreement with those of reproduction theory. In line with the maximally maintained inequality theory (MMI), a strong form of reproduction theory (Raftery and Hout, 1993), the results can be interpreted in the following way: Although educational expansion improved less-privileged children's educational opportunities of over time, more-privileged children's demand for higher postsecondary education has not yet been saturated. The overall picture shows that average scholastic performance is highly correlated with social background, while social background's direct effects on final educational attainment are stable over time.

\subsubsection{Primary and secondary effects' relative importance using linear regression models}

Tables 3.3a-3.3d show the results for attending and completing school career stages for the three school cohorts. The unstandardized regression coefficients are statistically significant at 1 percent level. Each Model 1 in Tables 3.3a-3.3d reports social background's total effect on educational attainment at each stage. The coefficients indicate an increasing effect of social 
background over time. Within a school career, social background's total effects are higher at the completion point than at the entry of secondary and postsecondary levels.

Table 3.3a: OLS -Entering secondary education

\begin{tabular}{lcccccc}
\hline & \multicolumn{2}{c}{1977} & \multicolumn{2}{c}{1989} & \multicolumn{2}{c}{1993} \\
& $(1)$ & $(2)$ & $(1)$ & $(2)$ & $(1)$ & $(2)$ \\
\hline Parents' education & $0.109^{* *}$ & $0.049^{* *}$ & $0.118^{* *}$ & $0.051^{* *}$ & $0.129^{* *}$ & $0.051^{* *}$ \\
CITO test & & $0.039^{* *}$ & & $0.034^{* *}$ & & $0.041^{* *}$ \\
Intercept & $5.911^{* *}$ & $4.379^{* *}$ & $5.607^{* *}$ & $4.508^{* *}$ & $5.437^{* *}$ & $4.003^{* *}$ \\
Observations & 28612 & 28612 & 17485 & 17485 & 15809 & 15809 \\
Adj. R squared & 0.152 & 0.551 & 0.181 & 0.541 & 0.175 & 0.593 \\
\hline
\end{tabular}

** $\mathrm{p}<0.01$ Source: CBS; own calculations.

Table 3.3b: OLS - Completing secondary education given secondary education attended

\begin{tabular}{|c|c|c|c|c|c|c|c|c|c|}
\hline & \multicolumn{3}{|c|}{1977} & \multicolumn{3}{|c|}{1989} & \multicolumn{3}{|c|}{1993} \\
\hline & (1) & $(2)$ & (3) & (1) & (2) & (3) & (1) & (2) & (3) \\
\hline Parents' education & $0.183^{* *}$ & $0.094 * *$ & $0.033 * *$ & $0.195 * *$ & $0.089 * *$ & $0.019 * *$ & $0.206 * *$ & $0.092 * *$ & $0.022 * *$ \\
\hline CITO test & & $0.063^{* *}$ & $0.015^{* *}$ & & $0.057^{* *}$ & $0.010^{* *}$ & & $0.063^{* *}$ & $0.010^{* *}$ \\
\hline $\begin{array}{l}\text { Controlled for school track } \\
\text { previously attended }\end{array}$ & & no & yes & & no & yes & & no & yes \\
\hline Intercept & $9.725^{* *}$ & $7.091 * *$ & $12.918 * *$ & $9.126 * *$ & $7.193^{* *}$ & $13.576^{* *}$ & $8.954 * *$ & $6.687 * *$ & $13.411^{* *}$ \\
\hline Observations & 24919 & 24919 & 24919 & 15804 & 15804 & 15804 & 14603 & 14603 & 14603 \\
\hline Adj. R squared & 0.137 & 0.472 & 0.714 & 0.166 & 0.504 & 0.825 & 0.168 & 0.537 & 0.838 \\
\hline
\end{tabular}

** $\mathrm{p}<0.01$ Source: CBS; own calculations.

Table 3.3c: OLS - Attending postsecondary education given secondary education completed

19771989

$\begin{array}{llllll}1 & (2) & (3) & \text { (1) }\end{array}$

(3)

\begin{tabular}{lcccccccccc}
\hline Parents' education & $0.128^{* *}$ & $0.078^{* *}$ & $0.019^{* *}$ & $0.138^{* *}$ & $0.074^{* *}$ & $0.019^{* *}$ & $0.145^{* *}$ & $0.076^{* *}$ & $0.018^{* *}$ \\
$\begin{array}{l}\text { CITO test } \\
\begin{array}{l}\text { Controlled for school track } \\
\text { previously completed }\end{array}\end{array}$ & & $0.043^{* *}$ & $0.004^{* *}$ & & $0.040^{* *}$ & $0.005^{* *}$ & & $0.044^{* *}$ & $0.004^{* *}$ \\
Intercept & & no & yes & & no & yes & & no & yes \\
Observations & $11.377^{* *}$ & $9.295^{* *}$ & $14.550^{* *}$ & $10.862^{* *}$ & $9.285^{* *}$ & $14.532^{* *}$ & $10.890^{* *}$ & $9.075^{* *}$ & $14.752^{* *}$ \\
Adj. R squared & 14650 & 14650 & 14650 & 11272 & 11272 & 11272 & 9921 & 9921 & 9921 \\
\hline & 0.102 & 0.336 & 0.752 & 0.124 & 0.370 & 0.783 & 0.125 & 0.393 & 0.812 \\
\hline
\end{tabular}


Table 3.3d: OLS - Completing postsecondary education given postsecondary education attended

1977

(1) (2)
(3)

(1)
1993

(2)

(3)

(1)

(2)

(3)

\begin{tabular}{|c|c|c|c|c|c|c|c|c|c|}
\hline Parents' education & $0.178^{* *}$ & $0.111^{* *}$ & $0.021^{* *}$ & $0.206^{* *}$ & $0.119 * *$ & $0.039 * *$ & $0.204^{* *}$ & $0.116^{* *}$ & $0.039 * *$ \\
\hline CITO test & & $0.058 * *$ & $0.006 * *$ & & $0.054^{* *}$ & $0.009 * *$ & & $0.057 * *$ & $0.012^{* *}$ \\
\hline $\begin{array}{l}\text { Controlled for school track } \\
\text { previously attended }\end{array}$ & & no & yes & & no & yes & & no & yes \\
\hline Intercept & $13.262^{* *}$ & $10.500 * *$ & $18.042^{* *}$ & $12.666^{* *}$ & $10.512^{* *}$ & $17.455^{* *}$ & $12.897 * *$ & $10.532 * *$ & $17.244^{* *}$ \\
\hline Observations & 14234 & 14234 & 14234 & 11021 & 11021 & 11021 & 9670 & 9670 & 9670 \\
\hline Adj. R squared & 0.109 & 0.337 & 0.821 & 0.125 & 0.329 & 0.664 & 0.124 & 0.347 & 0.650 \\
\hline
\end{tabular}

** $\mathrm{p}<0.01$ Source: CBS; own calculations.

Model 2 in Tables 3.3a-3.3d shows the cumulative secondary effects controlling for school performance. At each stage, these effects are rather stable over time. Within a cohort, however, these effects almost double over the educational career, from some 0.050 for the transition to secondary education to some 0.115 for completing the postsecondary education track. The increase of secondary effects at later transitions is flawed, since Model 2 does not control for previous decisions on track placement or track completion. Model 3 includes previous tracks and shows direct secondary effects at completing secondary education and entering postsecondary education decreased, while they increased at completing postsecondary education. In sum, secondary effects at the first transition are fairly high and stable over time, but they decrease in magnitude at subsequent transitions, mediated by previous school decisions. Model 3's explanatory power has clearly improved by 30 to 40 percentage points, compared with Model 2's; that is, much of the variance is explained by constraints of previous decisions.

Table 3.3e shows the results for the unconditional model. The coefficients are higher in magnitude than in the conditional model. However, their direction over time does not differ substantially from those of the conditional model. The unconditional model confirms a rather stable secondary effects' impact over time, while the results for the total effect of social stratification indicate an increase over time in all models. 
Table 3.3e: OLS - Final education (unconditional model), for those who attained a certificate in higher postsecondary education

\begin{tabular}{lcccccc}
\hline & \multicolumn{3}{c}{1977} & & & 1993 \\
& $(1)$ & $(2)$ & $(1)$ & $(2)$ & $(1)$ & $(2)$ \\
\hline $\begin{array}{l}\text { Parents' education } \\
\text { CITO test }\end{array}$ & 0.265 & 0.156 & 0.287 & 0.150 & 0.308 & 0.165 \\
$\begin{array}{l}\text { Controlled for school track } \\
\text { previously attended }\end{array}$ & & 0.077 & & 0.073 & & 0.079 \\
$\begin{array}{l}\text { Intercept } \\
\text { Observations }\end{array}$ & 10.647 & 7.425 & 10.544 & 8.060 & 10.332 & 7.498 \\
Adj. R squared & 24738 & 24738 & 15693 & 15693 & 14411 & 14411 \\
\hline
\end{tabular}

Note: All coefficients are significant at the $1 \%$ level. All students are considered who entered a secondary education track at age 12 and attained an official degreein their highest educational track observable. Source: CBS; own calculations.

Table 3.4 shows primary and cumulative secondary effects' relative importance, according to the OLS models. Secondary effects' relative impact is rather strong at the beginning of secondary education but diminishes over time, from $45 \%$ to $40 \%$. As a result, the cumulative secondary effects at completing the postsecondary education track, both in the conditional and unconditional models, also diminish over time, from $62 \%$ to $57 \%$ and from $59 \%$ to 56\%, respectively. Throughout the school career, cumulative secondary effects increase over its different stages, by some 16 percentage points. This illustrates that even at the school career's later stages secondary effects remain important and have a cumulative effect outweighing primary effects.

Table 3.4: OLS: Relative importance of primary and cumulative secondary effects on educational transitions and completions

\begin{tabular}{|c|c|c|c|c|c|c|}
\hline & \multicolumn{2}{|c|}{1977} & \multicolumn{2}{|c|}{1989} & \multicolumn{2}{|c|}{1993} \\
\hline & primary & secondary & primary & secondary & primary & secondary \\
\hline t1: enter second. education & 55 & 45 & 57 & 43 & 60 & 40 \\
\hline t2: complete second. education & 49 & 51 & 54 & 46 & 55 & 45 \\
\hline t3: enter post-second. education & 39 & 61 & 46 & 54 & 48 & 52 \\
\hline $\begin{array}{l}\text { t4: complete post-second. education } \\
\text { Final degree, unconditional from }\end{array}$ & 38 & 62 & 42 & 58 & 43 & 57 \\
\hline previous tracks & 41 & 59 & 48 & 52 & 46 & 54 \\
\hline
\end{tabular}


Similar to the counterfactual model's results, the OLS models' findings support both Hypotheses 1 and 3: Primary effects are more important at the first transition (H1), and they become more important over time at both transitions (H3). In contrast to the counterfactual model, we also find clear support for the other two hypotheses. The secondary effects are more important at the second transition (H2), while completing each stage becomes more meritocratic over time (H4).

\subsubsection{Primary and secondary effects' relative importance using structural equation models (LISREL)}

In a first step, we performed two analyses on the transition and attainment processes, controlling for measurement error only in school performance tests. Figure 3.3 shows the path model with the first track in secondary education as the dependent variable, and Figure 3.4 with the final education degree as the dependent variable. ${ }^{15}$ For illustration purposes, we present the results for the two models for cohort $1993 .^{16}$

All models show an acceptable fit. The root mean square of error approximation (RMSEA) is lower than 0.05 for all models, which is an indication of a good model fit (Browne and Cudeck 1993), while the chi-square is not significant for the models of cohorts 1989 and 1993. This indicates a good fit between data and models. ${ }^{17}$

\footnotetext{
${ }^{15}$ We also performed an additional analysis in which we include both first choice in secondary education and final obtained degree, but this does not yield substantially different results. Note that a conditional model over all transitions is not applicable here. The high correlations between the educational career's four stages impair a robust simultaneous estimation in LISREL.

${ }^{16}$ The findings of the path models of cohort 1977 and cohort 1989 are available on request.

${ }^{17}$ The only exception is the chi-square for cohort 1977, which is significantly different from zero (First choice: chi-square $=13.19, d f=1, p<0.01$; Final degree: chi-square $=24.70, d f=1, p<0.01$ ). This indicates the assumption that the model of cohort 1977 is not significantly different from the one in the population does not hold. We have decided not to change the model for this cohort, however, as it fits the data of the other two cohorts quite well, and it is theoretically sound and straightforward. Moreover, the RMSEA indicates a good model fit. We will exercise caution, though, with interpreting results for cohort 1977.
} 
Figure 3.3: Structural equation model (with latent variable cognitive skills) on transition to secondary education, school cohort 1993
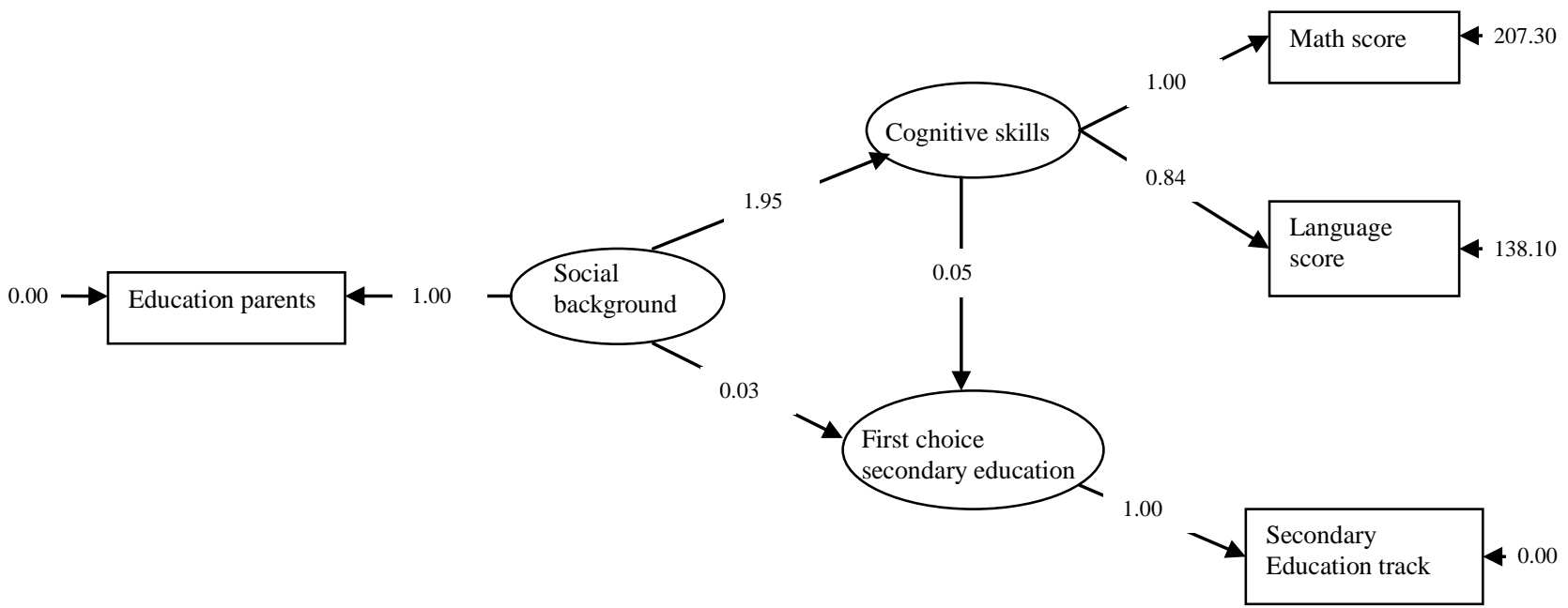

Chi-Square $=0.43, \mathrm{df}=1, \mathrm{P}$-value $=0.51025, \mathrm{RMSEA}=0.000$

Primary effects:

Secondary effects:

Total effect:

0.03
$76.5 \%$

$23.5 \%$

Figure 3.4: Structural equation model (with latent variable cognitive skills) on final educational degree attained, school cohort 1993
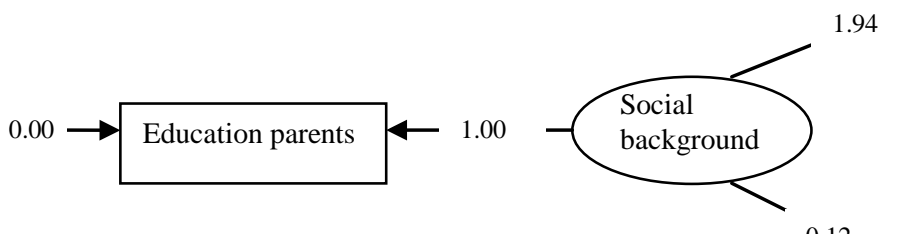

0.12

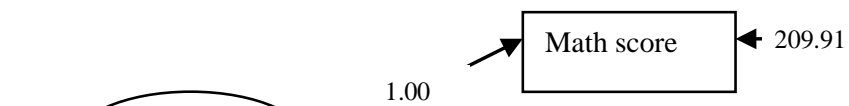

Chi-Square=1.18, $\mathrm{df}=1, \mathrm{P}$-value $=0.27638$, RMSEA $=0.004$

Primary effects:

Secondary effects:

Total effect:
0.12

$$
1.94 * 0.10=0.1940
$$

$0.1940+0.12=0.314$
$62.8 \%$

$38.2 \%$ 
The LISREL results obtained for the first transition (Figure 3.3) indeed reveal clear differences, compared with results obtained using the former methods. Primary effects are much more important than secondary effects if one accounts for measurement error in the performance tests: $76 \%$ of the social background effect is due to performance differences, compared with 24\%, which is related to differential choices. Over time, the relative importance of secondary effects at the first transition has slightly declined from $28 \%$ to $24 \%$ (see Table 3.5). Even if we omit the results of cohort 1977, this decrease is clearly visible.

Figure 3.4 shows that choice-related differences continue to have an effect. The cumulative secondary effects on the final obtained degree amount to $38 \%$. Over time, the relative importance of secondary effects on the final obtained degree also seems to decrease, although this holds when we compare only the oldest cohort with the two younger cohorts. Bearing in mind the model fit for cohort 1977 is not optimal, we can at least state secondary effects remained stable over the two last cohorts.

In a second step, we also control for measurement error in the latent variable social background by using both father's and mother's educational levels as separate indicators. Figure 3.5 shows the path model for cohort 1993 when the first transition to secondary education is made, while Figure 3.6, the path model for cohort 1993 on the final education degree. Again, we have good model fits for all our models of cohorts 1989 and $1993 .^{18}$

The first observation we make when comparing the parameter estimates in Figures 3.5 and 3.6 with those of Figures 3.3 and 3.4 is that social background's effects clearly increase. The effect on cognitive skills increases from 1.95 to 3.26, the effect on first choice increases from 0.03 to 0.06 , and the effect on final degree increases from 0.12 to 0.24 . This means the previous models seriously underestimated social background's total effects-both primary and secondary.

Compared with Figure 3.3, the path model of Figure 3.5 indicates secondary effects' relative importance is somewhat higher, $27 \%$ at the first transition. Secondary effects' higher relative magnitude is also displayed for the other two cohorts at the first transition (see Table 3.5), while they show a more apparent decrease from $37 \%$ to $27 \%$ over time (or from $31 \%$ to $27 \%$ if we omit cohort 1977).

\footnotetext{
${ }^{18}$ The exception is cohort 1977, with a chi-square for the first transition of 19.06, $d f=3, p<0.01$; and for the final degree with a chi-square of 27.76, $d f=3, p<0.01$.
} 
Figure 3.5: Structural equation model (with latent variable cognitive skills and social background) on transition to secondary education, school cohort 1993

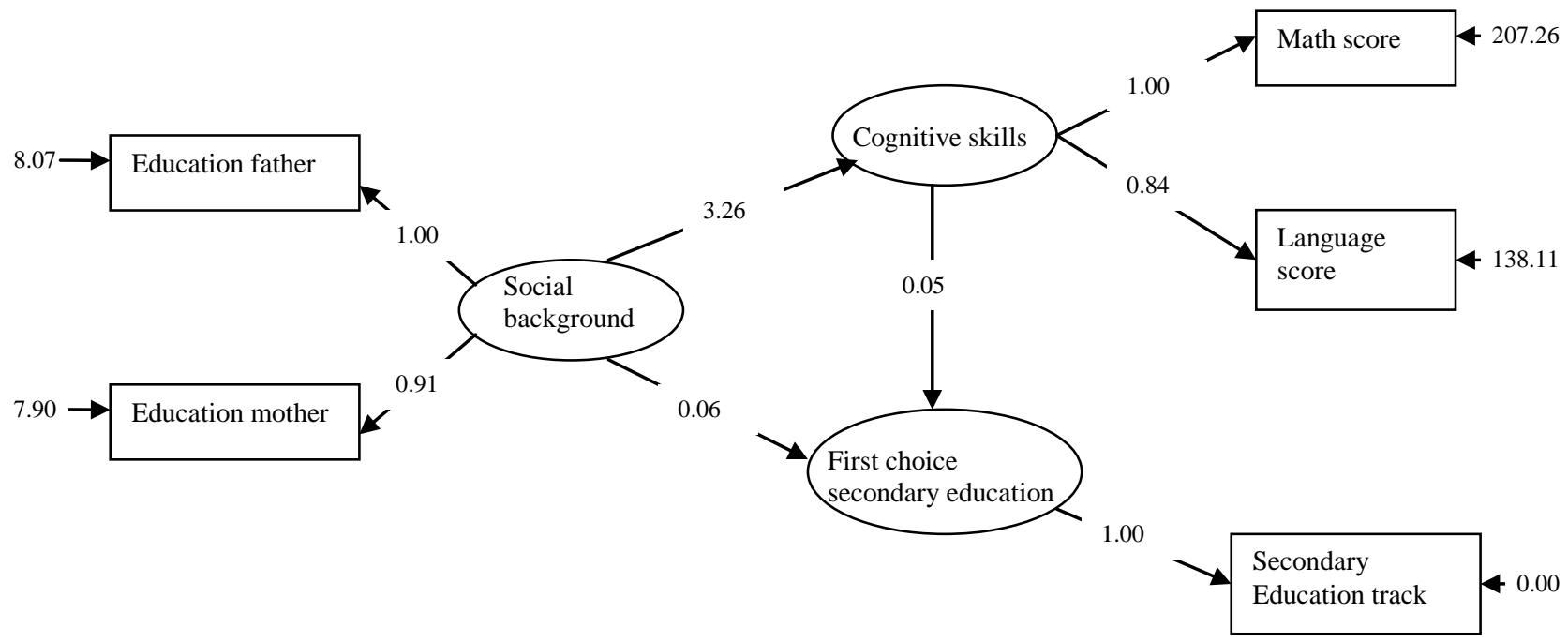

Chi-Square $=2.32, \mathrm{df}=3$, P-value $=0.50784$, RMSEA $=0.000$

Primary effects:

Secondary effects: $\quad 0.06$ Total effect:

$$
3.26 * 0.05=0.1940
$$

$0.163+0.06=0.223$
$73.1 \%$

$26.9 \%$

Figure 3.6: Structural equation model (with latent variable cognitive skills and social background) on final educational degree attained, school cohort 1993

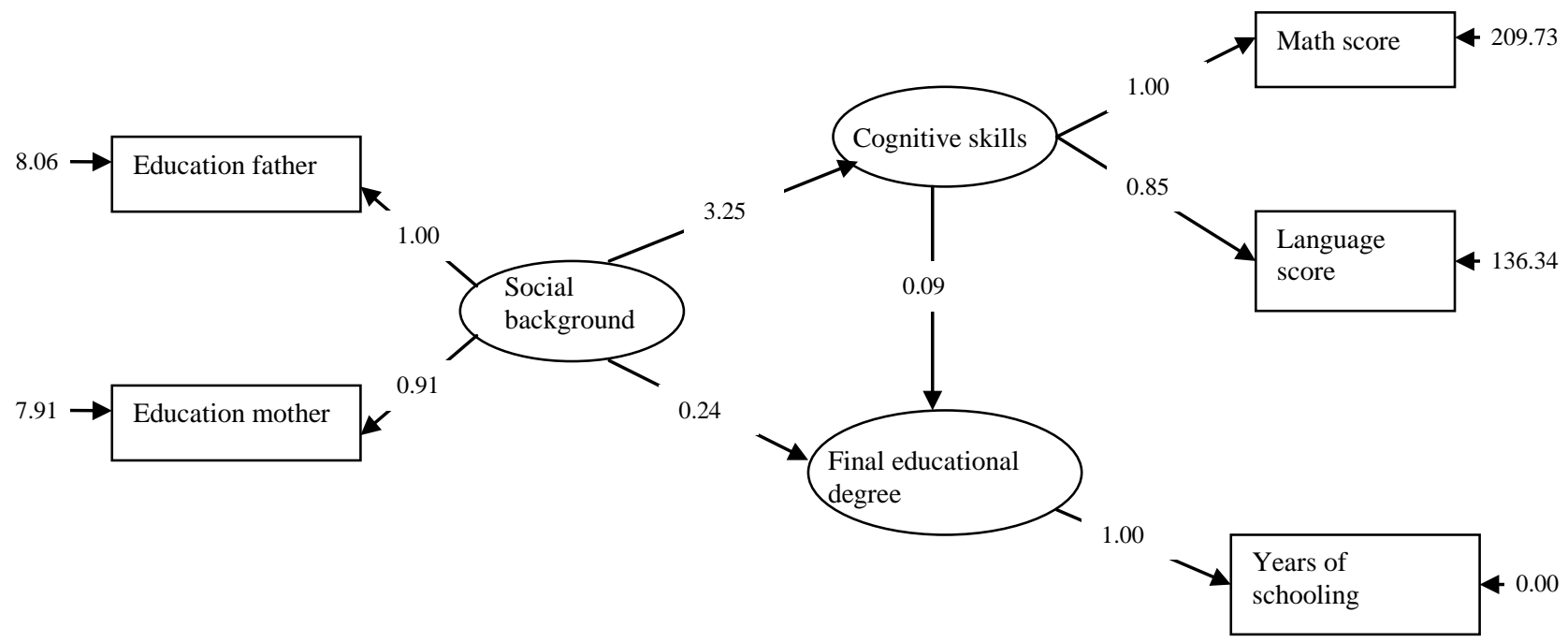

Chi-Square=3.19, $\mathrm{df}=3$, P-value $=0.36358$, RMSEA $=0.002$

Primary effects:

Secondary effects:

Total effect:
0.24

$3.25 * 0.09=0.2925$

$0.2925+0.24=0.5325$
$54.9 \%$

$45.1 \%$ 
Similar to Figure 3.4, Figure 3.6 shows that secondary effects increase again when we examine the final educational degree. However, the relative importance of secondary effects exceeds that of Figure 3.4 and amounts to $45 \%$, lower than the estimations of both the counterfactual and linear regression models. Regarding changes over time, the findings confirm a decrease when cohort 1977 is taken into account or at least a stable pattern in secondary effects' relative importance when considering only the two younger cohorts (see Table 3.5).

The LISREL results strongly confirm our first hypothesis, showing that primary effects are much more important than secondary effects at the first transition. At the stage of highest educational degree attained, while secondary effects' importance rises, primary effects remain relatively more important. Although this is not a direct test of the second hypothesis, it surely does not indicate that secondary effects at later stages are strong enough to offset the primary effects at the first transition, as they did in the counterfactual and linear regression models. Comparing the results over time (see Table 3.5), we find support for the modernization hypothesis, indicating more meritocratic selection for the first transition. For the final educational attainment, results indicate more meritocratic selection only if we examine the difference between the oldest and the two youngest cohorts. As indicated above, however, the results for cohort 1977 should be interpreted with caution. We therefore conclude the modernization hypothesis is not confirmed for the later stages.

That secondary effects' importance rises cumulatively at the final educational stage weakens modernization theory and the expectation of a clear performance-related process. Secondary effects may no longer dominate, after controlling for measurement error, but their relatively high share at the last stage indicates parents' direct impact on educational attainment at least does not decline as the child ages. This is in accordance with Boudon's assumption on economic constraints and related preferences and risks. ${ }^{19}$

Moreover, the path model in Figure 3.6 indicates that social background's "true" effect on cognitive skills is substantially higher than estimated in the previous LISREL model, without correcting for measurement error in social background. It suggests social background's overall effect is underestimated in linear regression models, and that including father's and mother's occupational status may yield even stronger social background effects on a child's educational performance and attainment.

\footnotetext{
19 Boudon (1974) argues that secondary effects have an equal share at each transition. They thus increase cumulatively until the last educational stage.
} 


\subsubsection{Comparing the findings from the three statistical methods}

Table 3.5 summarizes the proportions of secondary effects' relative importance obtained by the different statistical methods. If we compare the OLS results with the results from the counterfactual model, we find much consistency in the proportions. At the first transition to secondary education (t1), the OLS results reproduce the same or similar average values as in the counterfactual analysis. In addition, for the unconditional model with the final degree, the results do not differ significantly, with a slight exception for cohort 1977, which shows a higher share of secondary effects in the OLS model, compared with the counterfactual model. Secondary effects amount to some 55\% of social background's total effect if one uses linear regression or counterfactual models. The main difference between the two models concerns secondary effects' importance when entering postsecondary education. At this point, the OLS model consistently produces higher estimates than the counterfactual model does.

The largest differences are found-not unexpectedly_-between the LISREL model and the two other models. The results clearly indicate two conclusions. One is that social background's overall effects are underestimated if one does not properly control for measurement error in the social background indicators. The other conclusion is secondary effects' relative importance is overestimated if one does not properly control for measurement error in the tests assessing cognitive skills. For the two youngest cohorts, the difference between the counterfactual model and the OLS model amounts to about 10 percentage points for both the first transition and the final obtained degree. Using structural equation models, secondary effects' relative importance declines to some 45\%. Furthermore, the decrease over time in secondary effects at the first transition appears to be stronger in the structural equation model than in the two other models (10\% compared with 5\%).

The findings of all statistical methods show secondary effects' relative importance at the first transition has decreased over time. For the final obtained degree, most of the models display a high stability over time, with the exception of cohort 1977 in the LISREL model, which we should treat with some caution. This would mean that although secondary effects have decreased at the first transition, the total cumulative effect demonstrates considerable stability over time. This is partly caused by secondary effects' increasing at later stages. The OLS models show in the last stage, at the successful completion of postsecondary education, direct secondary effects of social stratification exhibiting the opposite trend, and increasing over time. 
3 Social background's effect on educational attainment: Does method matter?

Table 3.5: Summary of the relative importance of direct and cumulative secondary effects, obtained by different statistical methods, in \%

\begin{tabular}{|c|c|c|c|c|c|c|}
\hline & \multicolumn{2}{|c|}{ cohort 1977} & \multicolumn{2}{|c|}{ cohort 1989} & \multicolumn{2}{|c|}{ cohort 1993} \\
\hline & Direct & Cumulative & Direct & Cumulative & Direct & Cumulative \\
\hline \multicolumn{7}{|l|}{ Counterfactual model } \\
\hline t1: enter secondary education & & 45 & & 42 & & 41 \\
\hline t2: complete secondary education & & 54 & & 46 & & 45 \\
\hline t3: enter post-secondary education & & 51 & & 46 & & 45 \\
\hline t4: complete post-secondary education & & 55 & & 56 & & 55 \\
\hline t5: final degree, unconditional transition to higher education & & 55 & & 53 & & 55 \\
\hline \multicolumn{7}{|l|}{ Linear regression model } \\
\hline t1: enter secondary education & 45 & 45 & 43 & 43 & 40 & 40 \\
\hline t2: complete secondary education & 18 & 51 & 10 & 46 & 11 & 45 \\
\hline t3: enter post-secondary education & 15 & 61 & 14 & 54 & 12 & 52 \\
\hline t4: complete post-secondary education & 12 & 62 & 19 & 58 & 19 & 57 \\
\hline t5: final degree, unconditional transition to higher education & & 59 & & 55 & & 56 \\
\hline \multicolumn{7}{|l|}{ Structural equation model (latent variable for 'cognitive skills') } \\
\hline t1: enter secondary education & & 28 & & 27 & & 24 \\
\hline t5: final degree, unconditional transition to higher education & & 46 & & 38 & & 38 \\
\hline \multicolumn{7}{|l|}{$\begin{array}{l}\text { Structural equation model (latent variables for 'cognitive skills' } \\
\text { and 'social background') }\end{array}$} \\
\hline t1: enter secondary education & & 37 & & 31 & & 27 \\
\hline t5: final degree, unconditional transition to higher education & & 52 & & 46 & & 45 \\
\hline
\end{tabular}




\subsection{Conclusion}

This chapter has examined the relative importance of primary and secondary effects of social stratification at different stages during the educational career using data from three school cohorts in the Netherlands. An important analytical aspect was the use of three different statistical models to test for the findings' robustness. In this section, we will divide the discussion of our conclusion into two parts—substance and method.

Substantively, the findings of all our methods show a decreasing impact of secondary effects at the first transition over time. At later stages of the educational career, secondary effects' impact is rather stable and in some cases slightly increases. As a result, the cumulative share of choice-related differences in social background's effect on educational attainment is stable over time, at least if one examines the last two cohorts. The decrease in secondary effects at the first transition is consistent with modernization theory assumptions, which suggest an overall higher demand for education for less-privileged children. However, at later stages, secondary effects increase again; that is, the increased enrollment in higher education has not led to a decrease in secondary effects. Moreover, these secondary effects are even more important when completing a certain track than when entering a track, which offers a compelling argument for distinguishing between these two types. Arguments of rational action theory and reproduction theories support this tendency and suggest that reducing IEO at later stages in the educational career is difficult to achieve. Educational decisions seem to result from individual rational behavior where the utility of education is a function of (previous) educational performance and class-specific socioeconomic expectations. Or, as Boudon concludes, individuals "behave within decisional fields whose parameters are a function of their position in the stratification system” (1974:36). Regarding the overall picture of our findings, we therefore contradict modernization theory. Instead, we conclude educational expansion and increased meritocratic selection did not (fully) produce one or more declines in the associations among social background, cognitive performance, grade progression, and grade completion.

An important contribution of this analysis is offered in its comparing the results using three different statistical methods: counterfactual, linear regression, and structural equation models. As indicated in Section 3.3, these models differ in their conception of IEO, social background and educational attainment concepts, the use of conditional or unconditional models, and the treatment of measurement error. 
Starting with measurement error, our results show both the counterfactual and linear regression models are vulnerable to a correct measurement of cognitive skills and social background. On the one hand, they underestimate social background's overall effects, because they do not properly control for measurement error in the social background indicators. On the other hand, they overestimate secondary effects' relative importance, because they do not properly control for measurement error in the tests assessing cognitive skills. Both the counterfactual and the linear regression models seem to indicate primary and secondary effects are almost equal in size, with only small fluctuations over time. The structural equation model, in contrast, yields lower and more strongly declining secondary effects at the first transition over time.

The use of these three methods also implicitly reflects a different treatment of concepts and mechanisms, both with respect to the concept of IEO, and social background and educational attainment concepts. These differences can best be understood by comparing results from the counterfactual and the linear regression models. In practice, however, these two models basically produce the same results. This means the linear model does not add much over and above the counterfactual model, meaning the latter's results are appropriate in describing education selection processes. On the other hand, neither is it detrimental to use the linear model; to its advantage, it yields more detailed information on how postsecondary education selection occurs.

Finally, using conditional and unconditional models can provide more information concerning the educational system's function and influence. The conditional model is more in line with a view on the educational system as a form of contest mobility (Turner, 1960). The unconditional model considers the educational system more as a permeable strata system providing alternative pathways to higher education throughout the school career. Secondary effects' overall importance in the unconditional model resembles secondary effects' relative importance in the conditional model for completing postsecondary education. This illustrates there are no major alternative pathways in the educational system that children from lowereducated parents can pursue to remediate or otherwise improve on their earlier educational choices. We thus conclude the conditional model better reflects the dominant pathways students take, as they proceed through the education system in the Netherlands.

In sum, the most important conclusion is researchers should be aware that both linear regression and counterfactual models systematically underestimate social background's overall effect and overestimate choice-related differences' relative importance and thereby the type and potential of certain policy measures. Methods, such as structural equation 
modeling, which account for measurement error, are a necessary tool to reveal biases in the empirical information and to re-adjust conclusions for policy implications; that is, the structural equation model's findings relativize, to some extent, the size of secondary effects. Nevertheless, they do not contradict counterfactual and linear regression models' findings, with regard to secondary effects' relative importance later in the career. All models show that secondary effects increase at the end of education, relative to the first transition. On the one hand, for policy makers, this implies that policy actions implemented to decrease social inequality at the first transition were successful. Such endeavors should be continued and also extended to the area of performance-related differences at this stage. Early interventions can be effective in guiding children from lower social classes toward and onto academic tracks and-in the long run-potentially prevent deserving and well-qualified students from enduring social deprivation, unemployment, and other distressing social concerns on reaching adulthood.

On the other hand, unequal educational opportunities reoccur at later stages in the educational career. These may not be as life-altering as those experienced at the first transition, but as they are cumulative, their significance accrues over time. This suggests preferences and expectations of aspirants to higher educational levels are still strongly associated with social background. As these differences primarily refer to choice-related differences, more attention must be paid to combating this subtle form of IEO at the start of, and throughout, the postsecondary educational career. 
4 Education, cognitive skills, and earnings of males and females 


\subsection{Introduction}

Many empirical studies found evidence that cognitive skills have a positive impact on labor market outcomes of male workers (e.g. Herrnstein and Murray, 1994; Murnane, Willet and Levy, 1995). ${ }^{1}$ Cognitive skills affect earnings indirectly as they determine educational attainment and directly, as more able workers may be more productive since they have better problem-solving skills and are better able to perform more complex tasks (Bound, Griliches and Neumark, 1986; Zax and Rees, 1998; Currie and Thomas, 1999; Plug, Van Praag, and Hartog, 1999). Investments in human capital, such as in schooling and cognitive skills are rewarded on the labor market and assumed to have similar effects for males and females (Becker, 1964). However, different specializations at school or on the job, as well as different preferences in labor market behavior (Becker, 1974) may lead to different outcomes for males and females. ${ }^{2}$ Some scholars found that males significantly differ from females in mathematical skills and in the preference for technical study fields which indirectly triggers other endowments or investments in schooling that are rewarded at the labor market (Eccles, 1986; Schmader, 2002; Brown and Corcoran, 1997; Ellison and Swanson, 2010; Niederle and Vesterlund, 2010; Pope and Sydnor, 2010). ${ }^{3}$ Studies that examine the direct relationship between cognitive skills and labor market outcomes of males and females, however, show mixed results. Cawley, Coneely, Heckman, and Vytlacil (1996) find that ability is not rewarded equally across race and gender; Heineck and Anger (2008) find that cognitive ability only affects wages of male workers. Other studies find positive, comparable effects both for males and females (Arcidiacono, 2004; Heckman, Stixrud, and Urzua, 2006).

This study aims to analyze the indirect and direct earnings returns to cognitive skills for males and females in the Netherlands at age 35. We use a large unique data set which contains three proxies for cognitive skills at age 12, two of which are school achievement tests in math and language, and one is an IQ test to estimate more general and largely innate abilities (Baltes, Staudinger, and Lindenberger, 1999). In a first step, we want to examine males' and females' study field choices and their final educational level attained, relative to

\footnotetext{
${ }^{1}$ This chapter is based on joint work with Wendy Smits and Rolf van der Velden.

${ }^{2}$ Booth and Nolen (2009) assume that gender differences in behaviour are a result of social learning rather than of inherent gender traits. Parents and the social environment are likely to affect children's gender identities that eventually lead to different preferences and considerations of opportunity costs of males and females in school and at the labor market (Eagly, 1987; Chodorow, 1989; Corcoran and Courant, 1989).

${ }^{3}$ Brown and Corcoran (1997), for instance, found that earnings differences can partly be explained by the fact that males and females choose different fields of study in college, even after controlling for performance. Males tend to choose more 'lucrative' fields of study that are linked to better paid occupations and industries.
} 
their cognitive performance at school. We assume students with higher cognitive skills are likely to choose more demanding subjects at school and to attain higher levels of education. However, it is also likely that there are gender-related differences in study field choices and educational selection - conditional on cognitive skills - which may be related to preferences or other, possibly institutional factors. In a second step, we investigate to what extent males and females are rewarded for their cognitive skills on the labor market, in general and conditional on their study fields and educational levels attained. In the Netherlands, for example, the students' study field choices and educational level attained are crucial components in screening school leavers and graduates at the labor market.

Our findings show significant gender differences in math and language performance, as well as in non-verbal intelligence. Cognitive skills, and especially math skills, strongly predict schooling outcomes for both gender, but better for males than for females. Moreover, males tend to choose 'more lucrative' study fields, such as engineering, once they attain higher levels of education. When running earnings regressions separately by gender, we find significant earnings returns to cognitive skills both for males and females. Math skills, once more, turn out to predict earnings better than language skills and IQ. This is also confirmed by other studies (e.g. Paglin and Ruffalo, 1990; Grogger and Eide, 1995; Murnane et al., 1995; Weinberger, 1999, 2001; Murnane, Willet, Duhaldeborde, and Tyler, 2000; Altonji and Blank, 1999; Arcidiacono, 2004). Specifying the earning regressions by the attained educational level, females' returns to math skills remain strong and significant at all educational levels. For males however, we only find a significant effect of math skills for the lower educated. Our findings suggest that there is a females' 'reserve of talent' when looking at educational attainment but this 'reserve' of cognitive skills is nevertheless rewarded on the labor market.

This chapter proceeds as follows: Section 4.2 gives an overview of the data, the variables operationalization, and the empirical strategy. Section 4.3 presents the descriptive statistics and the results of the multivariate analyses. Section 4.4 summarizes the findings and concludes.

\subsection{Data and model specification}

For the analysis we make use of a unique and rich data set, that is based on the so-called Secondary Education Pupils Cohort (SLVO) 1982 provided by Statistics Netherlands. This is a national representative longitudinal data set which contains information on some 16,800 
individuals who entered secondary education in 1982 and were followed throughout their school career until they left the education system.

In the first year of the survey, when the respondents were 12 years old, skills tests were conducted in math and language as well as a test on non-verbal intelligence. At the same time information on family background was gathered by interviewing the parents of the pupils. This comprises information on ethnicity and education of the parents, family composition and attitudes toward the school career of their children.

The respondents have been followed throughout their entire educational career. Annual information about their educational status (track, grade and diploma), until they left full-time education, is documented by schools records, which ensures a high quality of the data information. Moreover, the cohort does not exhibit serious panel attrition that could bias the results.

The cohort data have been enriched with administrative data, coming from municipalities and national tax registers providing information on labor force status and earnings of the respondents and their partners, as well as the family composition of the respondent's household (marital status, number and age of children) in 2005, at the time when the respondents were about 35 years old. The fact that these data are from registers, provides a high reliability, especially of the earnings data.

We restricted the sample to males and females who were employed and who did not work in 2005. The final data set comprises 13,337 individuals, which is about $80 \%$ of the original survey population. ${ }^{4}$ Earnings information is captured for about 5,800 male and 5,200 female employees. This number is large enough to allow us to run separate models for males and females.

\section{Variables operationalization}

The variables used for the analyses are operationalized in the following way: The schooling information refers to the highest educational level attained and is calculated in years, on a scale ranging from 6 to 19 years. This is according to the 'years of schooling ladder' by Bosker et al. (1985), measuring the progress to the top of the Dutch education system (19 years for completing university). In a next step, we group this information into three categories of lower, medium, and higher educated individuals, following the International

\footnotetext{
${ }^{4}$ Traag, Van der Valk, Van der Velden, and de Vries (2006) show that the group for which a match could be made with administrative data is representative for the whole sample.
} 
Standard Classification of Education (ISCED). ${ }^{5}$ The following values were assigned: up to 12 years of schooling (lower education, ISCED 0-2), up to 15 years of schooling (medium education, ISCED 3-4), and up to 19 years of schooling (higher education, ISCED 5-6).

We use three proxies for estimating cognitive skills, all measured at age 12 . The math and language tests are based on the so-called CITO test, a nationwide standardized school performance test that is comparable to the SAT. Each test comprises 20 multiple choice questions. The sum-score is standardized with mean zero and standard deviation one. Cronbach's alpha for the math test is 0.80 and 0.70 for the language test (Bosman, 1993). Furthermore, a non-verbal intelligence test was done on logical reasoning and the capacity to abstract (Horn, 1969). The test comprises 40 items of which the sum-score again is standardized with mean zero and standard deviation one. Cronbach's alpha for this test is 0.79 (Bosman, 1993).

The study field variable refers to the study field observed at the highest educational level attained. We use six dummy variables assigned to: agricultural studies, economics studies, engineering studies, health studies, general studies, and other studies (social studies, humanities and arts).

Having a migration history is measured by the country of birth of both the respondent and one of his or her parents, at the start of the panel survey. Someone is considered to belong to an ethnic minority, if either the respondent or his or her parents were born in one of the non-western countries listed in the Employment of Minorities (Promotion) Act (Wet SAMEN), and is otherwise zero.

The dummy variable siblings indicates whether the respondent had siblings (1) or not (0), at the start of the panel survey at age 12.

The educational level of the parents was determined according to the Standard Education Classification 1978 (Centraal Bureau voor de Statistiek, 1987). We converted the different levels to the average number of years of schooling, according to the abovementioned scale by Bosker et al. (1985). The following values were assigned: 6 years (primary education, ISCED 0-1), 10 years (secondary education, lower level, ISCED 2), 14 years (secondary education, higher level, ISCED 3), 17 years (tertiary education, first phase, ISCED 5 bachelor) and 19 years (tertiary education, second phase, ISCED 5 master or ISCED 6).

\footnotetext{
${ }^{5}$ For a detailed description of the ISCED, see: http://www.uis.unesco.org/Education/Pages/internationalstandard-classification-of-education.aspx and http://circa.europa.eu/irc/dsis/employment/info/data/eu_lfs/lfs_main/Related_documents/ISCED_EN.htm
} 
The parental educational support is based on questions to both parents about having discussions about school, having discussions about school performance and giving compliments about school performance. These items were combined to provide a scale with a minimum value of 0 and a maximum value of 1 . The Cronbach's alpha is 0.61 .

From the register of the municipalities we obtain whether in 2005, at the time when the respondents were about 35 years old, he or she was living with a partner (dummy), the age of a partner, and the number and age of children in the household.

The labor force status of the respondent is measured as being in full-time, part-time or having no job in September 2005.

The earnings information of the respondent is based on the logarithm of average gross monthly earnings in September 2005. The earnings are standardized by means of a part-time factor, ranging from 0.1 to 1 full-time equivalent.

Having a higher educated partner comes from register data. The dummy takes value 1, if the partner has attained a degree at a vocational college (HBO) or at university (WO), and takes value 0 otherwise.

\section{Empirical strategy}

With regard to the earnings regressions, we first estimate the effect of non-verbal intelligence, math and language skills on earnings separate for all males and females. The earnings equation has the following form:

$$
\begin{aligned}
& Y_{i}=\beta_{0}+\beta_{1} M_{i}+\beta_{2} L_{i}+\beta_{3} I Q_{i}+\beta_{4} X_{i}+\varepsilon_{i} \\
& \text { with } E\left(\eta_{i} X_{i}\right)=0 \text {, where } \eta_{i}=\beta_{0}+\varepsilon_{i}
\end{aligned}
$$

where $Y_{i}$ are earnings of an individual $i . M_{i}$ denotes math skills, $L_{i}$ language skills and $I Q_{i}$ denotes non-verbal intelligence. $X_{i}$ is a vector of further observed controls and $\varepsilon_{i}$ the idiosyncratic error term.

In a second step, we allow for sorting into different labor markets, according to the different levels of lower, medium, and higher education. ${ }^{6}$ It is likely that cognitive skills are rewarded differently in different markets (cf. Heckman et al., 2006:421). The returns to cognitive skills can increase with schooling, if one assumes that skills are more important at

\footnotetext{
${ }^{6}$ Within the lower, medium, and higher education levels, we differentiate between sublevels (e.g. as between BA and MA/MSc for higher educated students), for which we control.
} 
higher job levels. At the same time, the returns to cognitive skills may differ depending on whether it is regarded as a substitute for or a predictor of schooling. In the first case, we might expect stronger effects of cognitive skills (especially at lower levels of education) than in the latter case. Equation 4.2. resembles equation 4.1, but will allow for separate rewards (at different labor markets) for employees of different educational levels $e$ :

$$
\begin{array}{r}
\ln Y_{i e}=\beta_{0}+\beta_{1 e} M_{i e}+\beta_{2 e} L_{i e}+\beta_{3 e} I Q_{i e}+\beta_{4 e} X_{i e}+\varepsilon_{i e} \\
\text { with } E\left(\eta_{i} X_{i e}\right)=0 \text {, where } \eta_{i}=\beta_{0}+\varepsilon_{i e}
\end{array}
$$

\subsection{Results}

\subsubsection{Descriptive statistics}

Table 4.1 presents some descriptive statistics. Starting with males’ and females' earnings, we note significant gender differences. In 2005, females' average log earnings were $20 \%$ lower than those of males, and this holds for each attained level of education (see Table 4.2). As to the labor force status, differences between males and females are also statistically significant. In $2005,81 \%$ of the males were in full-time employment, $11 \%$ were in part-time employment while $9 \%$ had no job (unemployed or out of the labor force). For females these figures were 23\% (full-time employed), 54\% (part-time employed), and 23\% (no job).

With regard to the cognitive skills proxies, we note that males on average have higher math scores than females, while females score higher in language and non-verbal intelligence tests. The difference in math scores is around 1/5 of a standard deviation. Although some studies found that the gender gap in achievement tests has narrowed over time (Hyde, Fennema, and Lamon, 1990; Ellison and Swanson, 2010; Niederle and Vesterlund, 2010; Pope and Sydnor, 2010), our findings in Table 4.2 show that differences in math, language and non-verbal intelligence tests between girls and boys at age 12 are statistically significant for this cohort and at each educational level.

With respect to years of schooling, we find hardly any gender difference (12.5 years for males and 12.6 years for females), but there are significant differences in study field choices. Table 4.2 shows that males dominate in engineering studies, while females represent the majority in health studies at all education levels. On average, more than a third of the males graduate in engineering, while only $6 \%$ of the females do so. In health studies, we find the opposite: one third of the females, and $2 \%$ of the males graduate in this field. We find equal 
shares of males and females in economics studies at the intermediate education level, while there are significantly more females than males at the lower education level, and significantly more males than females at the higher education level graduating in this field. ${ }^{7}$

Table 4.1: Summary statistics of the selected sample

\begin{tabular}{|c|c|c|c|c|c|c|}
\hline & \multicolumn{2}{|c|}{ Males } & \multicolumn{2}{|c|}{ Females } & \multirow[b]{2}{*}{ Min } & \multirow[b]{2}{*}{ Max } \\
\hline & Mean & SD & Mean & SD & & \\
\hline \multicolumn{7}{|l|}{ Cognitive skills and education } \\
\hline Math score & 0.118 & 1.008 & -0.112 & 0.985 & -1.728 & 1.730 \\
\hline Language score & -0.051 & 1.005 & 0.063 & 0.994 & -1.744 & 1.731 \\
\hline IQ score & -0.038 & 1.005 & 0.047 & 0.998 & -2.111 & 2.111 \\
\hline Years of schooling & 12.510 & 3.616 & 12.580 & 3.370 & 6 & 19 \\
\hline \multicolumn{7}{|l|}{ Field of study (Dummies) } \\
\hline Agriculture & 0.056 & 0.231 & 0.024 & 0.154 & 0 & 1 \\
\hline Engineering & 0.371 & 0.483 & 0.056 & 0.23 & 0 & 1 \\
\hline Health & 0.024 & 0.152 & 0.265 & 0.442 & 0 & 1 \\
\hline Economics & 0.174 & 0.379 & 0.176 & 0.381 & 0 & 1 \\
\hline Other & 0.058 & 0.234 & 0.091 & 0.287 & 0 & 1 \\
\hline General & 0.317 & 0.465 & 0.388 & 0.487 & 0 & 1 \\
\hline \multicolumn{7}{|l|}{ Family background } \\
\hline Having a migration history & 0.092 & 0.289 & 0.087 & 0.282 & 0 & 1 \\
\hline Having one or more siblings & 0.894 & 0.308 & 0.895 & 0.307 & 0 & 1 \\
\hline Years of schooling mother & 9.434 & 3.47 & 9.308 & 3.505 & 6 & 19 \\
\hline Years of schooling father & 10.334 & 4.076 & 10.254 & 4.073 & 6 & 19 \\
\hline Educ. support by parents & 0.514 & 0.285 & 0.490 & 0.274 & 0 & 1 \\
\hline \multicolumn{7}{|l|}{ Labor market characteristics } \\
\hline No job & 0.086 & 0.281 & 0.228 & 0.420 & 0 & 1 \\
\hline Part time employed & 0.105 & 0.306 & 0.541 & 0.498 & 0 & 1 \\
\hline Full time employed & 0.809 & 0.393 & 0.231 & 0.421 & 0 & 1 \\
\hline Log monthly earnings & 7.993 & 0.369 & 7.796 & 0.432 & 3.332 & 10.188 \\
\hline No child between 4-12 years & 0.717 & 0.451 & 0.521 & 0.500 & 0 & 1 \\
\hline One child between 4-12 years & 0.174 & 0.379 & 0.277 & 0.448 & 0 & 1 \\
\hline More children between $4-12$ years & 0.109 & 0.311 & 0.202 & 0.401 & 0 & 1 \\
\hline Child $\mid<4$ years in household & 0.391 & 0.488 & 0.450 & 0.498 & 0 & 1 \\
\hline Having a partner in 2005 & 0.725 & 0.446 & 0.799 & 0.401 & 0 & 1 \\
\hline Having a higher educated partner & 0.265 & 0.442 & 0.222 & 0.415 & 0 & 1 \\
\hline Partner has any kind of income & 0.628 & 0.483 & 0.780 & 0.414 & 0 & 1 \\
\hline Age of the partner & 32.537 & 3.917 & 36.691 & 3.877 & 16 & 80 \\
\hline
\end{tabular}

\footnotetext{
${ }^{7}$ Note that predicting cognitive skills' impact on study field choices and the attained educational level can be interrelated. General studies, for example, only exist at lower and medium education levels. Furthermore, there are two mechanisms at work; students, who went to prevocational education made their study field choices at age 12 (although they might have switched later on). Students, who went to general secondary education choose their study field, when they enter higher (or upper secondary vocational) education. As a result, we find that for lower educated males and females math skills have a negative impact on their choice for engineering (see Tables A4.1 and A4.2 in the appendix). Students with better math and language skills, however, are more likely to select in general secondary education. We therefore focus in our description on the study field choices at the higher educational levels, as the group is already selected on the basis of higher skills.
} 
4 Education, cognitive skills, and earnings of males and females

Table 4.2: Summary statistics of selected variables, males and females specified by educational level

\begin{tabular}{|c|c|c|c|c|c|c|c|c|c|}
\hline \multirow[b]{2}{*}{ Variable } & \multicolumn{2}{|c|}{ Lower education } & \multirow{2}{*}{$\begin{array}{c}\text { t-test } \\
p \text {-value }\end{array}$} & \multicolumn{2}{|c|}{ Medium education } & \multirow{2}{*}{$\begin{array}{c}\text { t-test } \\
\text { p-value }\end{array}$} & \multicolumn{2}{|c|}{ Higher education } & \multirow{2}{*}{$\begin{array}{c}\text { t-test } \\
p \text {-value }\end{array}$} \\
\hline & Males & Females & & Males & Females & & Males & Females & \\
\hline Log monthly earnings & 7.874 & 7.638 & 0.000 & 8.010 & 7.817 & 0.000 & 8.230 & 8.054 & 0.000 \\
\hline No job & 0.123 & 0.321 & 0.000 & 0.048 & 0.179 & 0.000 & 0.053 & 0.101 & 0.000 \\
\hline Part-time working & 0.095 & 0.493 & 0.000 & 0.095 & 0.586 & 0.000 & 0.142 & 0.564 & 0.000 \\
\hline Full-time working & 0.782 & 0.187 & 0.000 & 0.857 & 0.235 & 0.000 & 0.806 & 0.335 & 0.000 \\
\hline Math score & -0.332 & -0.516 & 0.000 & 0.369 & 0.038 & 0.000 & 0.820 & 0.583 & 0.000 \\
\hline Language score & -0.462 & -0.321 & 0.000 & 0.166 & 0.228 & 0.032 & 0.606 & 0.672 & 0.055 \\
\hline IQ score & -0.246 & -0.197 & 0.048 & 0.047 & 0.149 & 0.001 & 0.329 & 0.443 & 0.003 \\
\hline \multicolumn{10}{|l|}{ Field of study (Dummy): } \\
\hline - agriculture & 0.043 & 0.03 & 0.365 & 0.081 & 0.011 & 0.000 & 0.053 & 0.036 & 0.038 \\
\hline - economics & 0.048 & 0.082 & 0.000 & 0.259 & 0.235 & 0.249 & 0.353 & 0.285 & 0.000 \\
\hline - engineering & 0.397 & 0.050 & 0.000 & 0.290 & 0.038 & 0.000 & 0.42 & 0.115 & 0.000 \\
\hline - health & 0.006 & 0.215 & 0.000 & 0.035 & 0.348 & 0.000 & 0.049 & 0.206 & 0.000 \\
\hline - other & 0.063 & 0.068 & 0.073 & - & - & - & 0.126 & 0.358 & 0.000 \\
\hline - general & 0.442 & 0.555 & 0.000 & 0.335 & 0.368 & 0.000 & - & - & - \\
\hline One child between 4-12 years & 0.200 & 0.322 & 0.000 & 0.167 & 0.262 & 0.000 & 0.124 & 0.195 & 0.000 \\
\hline More children between 4-12 years & 0.132 & 0.240 & 0.000 & 0.104 & 0.197 & 0.000 & 0.061 & 0.115 & 0.000 \\
\hline Having a child $\mid<4$ years & 0.360 & 0.362 & 0.950 & 0.414 & 0.509 & 0.000 & 0.433 & 0.544 & 0.000 \\
\hline Having a partner & 0.706 & 0.770 & 0.000 & 0.736 & 0.831 & 0.000 & 0.755 & 0.800 & 0.007 \\
\hline Having a higher educated partner & 0.112 & 0.096 & 0.075 & 0.277 & 0.215 & 0.000 & 0.585 & 0.550 & 0.115 \\
\hline Partner has any kind of income & 0.595 & 0.749 & 0.000 & 0.651 & 0.816 & 0.000 & 0.676 & 0.782 & 0.000 \\
\hline Age of partner & 32.66 & 36.991 & 0.000 & 32.371 & 36.506 & 0.000 & 32.646 & 36.384 & 0.000 \\
\hline
\end{tabular}

Note: Differences in math, language and IQ-scores between males of different educational levels and between females of different educational levels are all significant.

${ }^{\mathrm{a}}$ The lower education level comprises ISCED 1 and 2; the medium education level comprises ISCED 3 and 4 and the higher education level ISCED 5 and 6. 


\subsubsection{Estimation results for males' and females' educational outcomes}

Table 4.3 reports our results of the three cognitive skills measures on predicting males' and females’ years of schooling.

Table 4.3: OLS on years of schooling, separate for males and females

\begin{tabular}{|c|c|c|c|c|}
\hline & \multirow[b]{2}{*}{$(1)$} & \multirow{2}{*}{$\begin{array}{l}\text { Males } \\
(2)\end{array}$} & \multicolumn{2}{|c|}{ Females } \\
\hline & & & (1) & $(2)$ \\
\hline \multirow[t]{2}{*}{ Math score } & $1.044 * * *$ & $0.961 * * *$ & $0.822 * * *$ & $0.716^{* * *}$ \\
\hline & $(0.049)$ & $(0.048)$ & $(0.047)$ & $(0.046)$ \\
\hline \multirow[t]{2}{*}{ Language score } & $0.824^{* * *}$ & $0.733^{* * *}$ & $0.641^{* * *}$ & $0.569 * * *$ \\
\hline & $(0.048)$ & $(0.047)$ & $(0.045)$ & $(0.044)$ \\
\hline \multirow[t]{2}{*}{ IQ score } & $0.215^{* * *}$ & $0.188 * * *$ & $0.289 * * *$ & $0.244 * * *$ \\
\hline & $(0.041)$ & $(0.041)$ & $(0.040)$ & $(0.039)$ \\
\hline \multirow[t]{2}{*}{ Schooling father } & & $0.371 * * *$ & & $0.324 * * *$ \\
\hline & & $(0.045)$ & & $(0.043)$ \\
\hline \multirow[t]{2}{*}{ Schooling mother } & & $0.245 * * *$ & & $0.225^{* * *}$ \\
\hline & & $(0.051)$ & & $(0.049)$ \\
\hline \multirow[t]{2}{*}{ Migration history } & & $-0.077 * * *$ & & $-0.696 * * *$ \\
\hline & & $(0.142)$ & & $(0.146)$ \\
\hline \multirow[t]{2}{*}{ Having siblings } & & 0.225 & & $0.373 * * *$ \\
\hline & & $(0.149)$ & & $(0.131)$ \\
\hline \multirow[t]{2}{*}{ Parents' educational support } & & 0.139 & & -0.054 \\
\hline & & $(0.141)$ & & $(0.136)$ \\
\hline $\mathrm{N}$ & 6425 & 6425 & 6912 & 6912 \\
\hline $\mathrm{R} 2$ & 0.237 & 0.267 & 0.175 & 0.211 \\
\hline
\end{tabular}

Note: $* * * \mathrm{p}<0.01 \quad * * \mathrm{p}<0.05 \quad * \mathrm{p}<0.10$; robust standard errors in parentheses.

We find the explained variance in years of schooling being higher for males than for females: 24\% versus 18\% (Model 1). All cognitive skills measures strongly predict schooling, but the magnitudes of both the math skills and language skills coefficients are higher for males and significant different from those for females. The results also hold, if we control for parents' schooling and educational support, the respondent's migration history, and whether he or she has siblings (Model 2).

Figures 4.1 and 4.2 confirm this in graphical form. They show the probability of males and females having a higher education degree at the different levels of the cognitive skills measures. The slopes for math and language scores of males show a steeper increase in the probability of graduating from a vocational college or university than those of females. 
Figure 4.1: Males’ probability of being graduated from college or university at cognitive skills

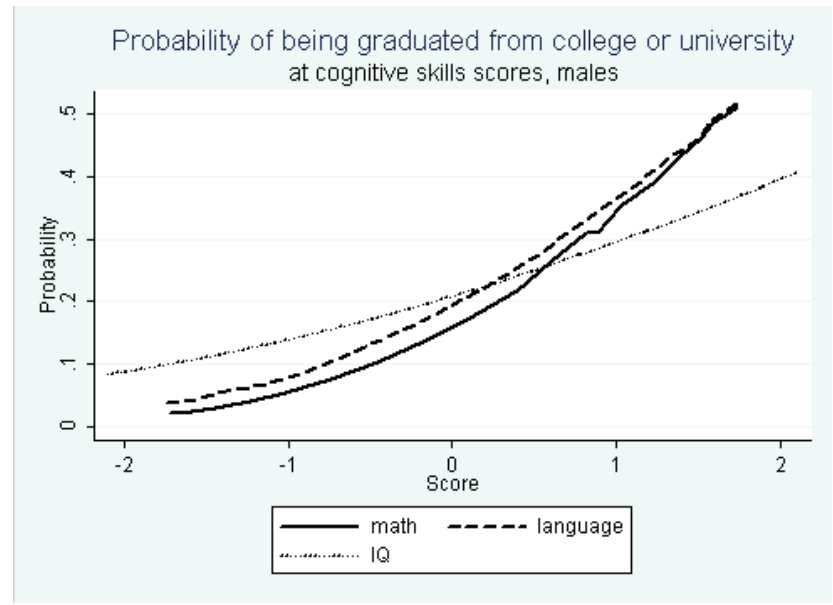

Figure 4.2: Females’ probability of being graduated from college or university at cognitive skills

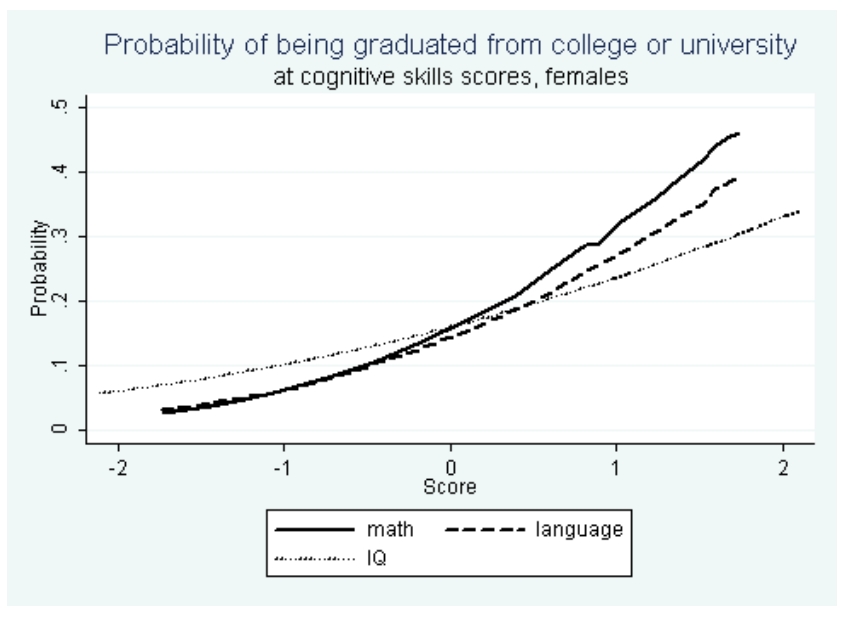

In Table 4.4 we examine the relationship between cognitive skills and study field choices, conditional on attained educational level. This table reports the predicted marginal effects of males' and females' graduating from a particular field of study in higher education. The full results for the other levels of education are shown in the appendix (Table A4.1 and A4.2).

Our findings indicate that males with high math scores more often choose engineering and less often 'other studies' (i.e. social studies, humanities and arts), while the reverse is true for high language skills. Females with high math scores are, similar to males, more likely to choose engineering, but at the expense of health studies. In contrast, females with high language scores more often graduate from an economics program and less often from engineering. 
Table 4.4: Multinomial logit regression on field of study choice of males and females in higher education (predicted marginal effects)

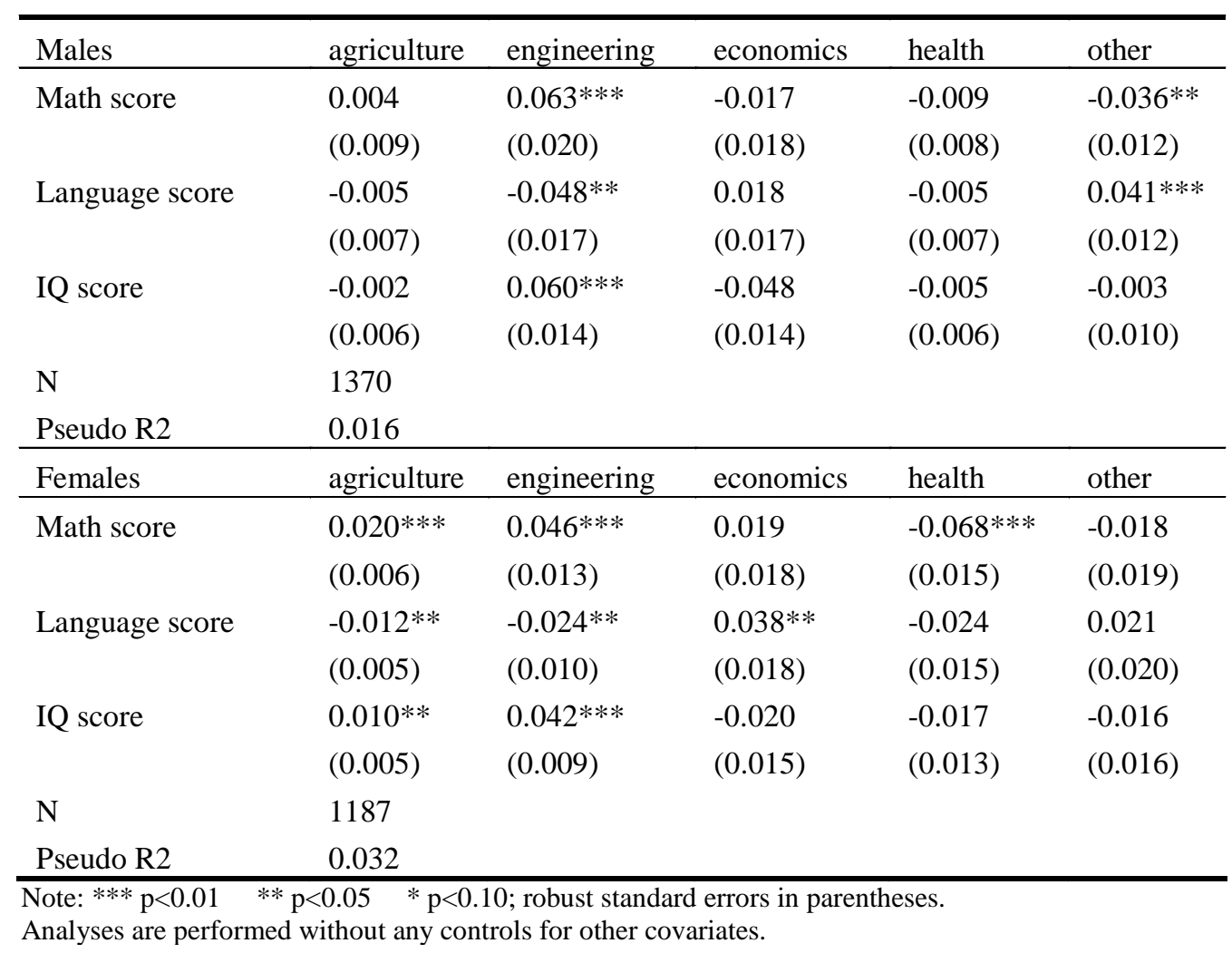

Figure 4.3 shows males' and females' predicted probabilities of graduating from an engineering program in higher education. The figure illustrates that the probability of graduating from such a program is increasing with higher math skills and this holds stronger for males than for females.

Figure 4.3: Probability of graduating from engineering studies at math scores, higher educated males and females

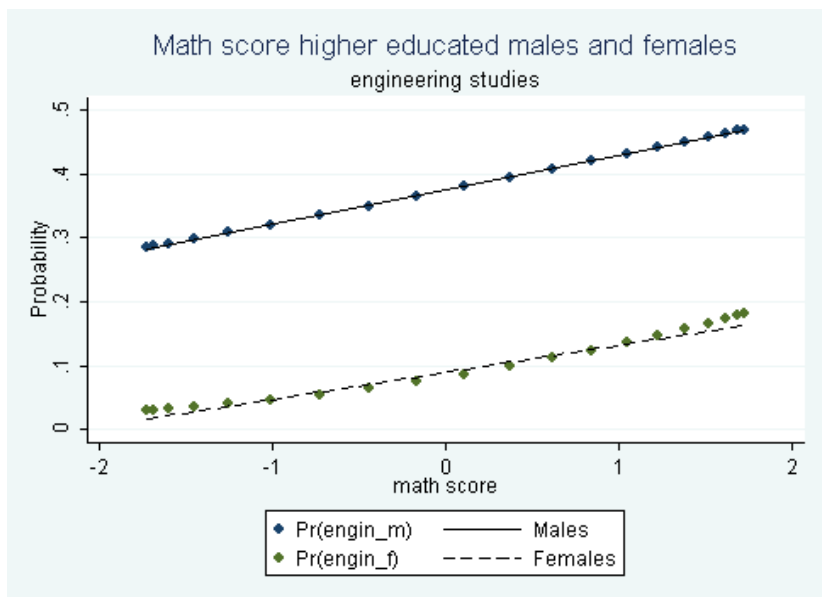




\subsubsection{Estimation results for males' and females' labor market outcomes}

Tables 4.5 and 4.6 present the results of the earnings regressions separately for all males and females. The first two columns present the model for the total group (eq. 4.1) and the other columns the results per level of education (eq. 4.2). The first column in the model for the total group shows the effect of cognitive skills without controls for schooling and background characteristics. Without controlling for other characteristics, cognitive skills explain some 7\% of the earnings of males and $9 \%$ of the earnings of females, with the strongest effects for math and languages skills. ${ }^{1}$

After controlling for schooling and field of study in the second model for the total group, the effects of cognitive skills decrease but the math skills still have an effect on earnings, both for males and females. An increase in math skills of one standard deviation is associated with a wage premium of $2.9 \%$ for males and $3.7 \%$ for females. ${ }^{2}$ While higher scores in language skills and non-verbal intelligence do not play a significant role for males’ earnings anymore after controlling for schooling, females still profit from it. An increase in females' language skills and non-verbal intelligence of one standard deviation is related to a wage premium of $2.6 \%$ and $1.2 \%$, respectively.

Including further controls for social background and the current family composition of the respondent in the third model of all males and females do not substantively change the values of the cognitive skills coefficients. ${ }^{3}$

The results show that the achievement tests have a greater predictive power than the IQ test in explaining earnings differentials. Almlund et al. (2011) and Borghans et al. (2011) explain this by the fact that achievement tests take up a substantial portion of personality indicators that play an important role in predicting life outcomes.

\footnotetext{
${ }^{1}$ The bivariate correlation between math scores and earnings is 0.29 for males and 0.27 for females. For languages scores these correlations are 0.24 and 0.25 respectively and for IQ scores the corresponding figures are 0.12 and 0.15. This is comparable to the estimates found in studies of Cameron and Heckman (1993) and Heckman et al. (2006), but they are smaller than the estimates in Neal and Johnson (1996) and Zax and Rees (2002).

${ }^{2}$ Note that this wage effect of math skills is related to schooling. As schooling is a choice variable and depends on cognitive skills, schooling is endogenous in the earnings equation (cf. Heckman et al., 2006). Therefore the returns to math skills are lower than in a model without schooling.

${ }^{3}$ For some control variables there is a higher number of missing values in the final sample, but they are not related systematically to cognitive skills or other (demographic) characteristics of the respondent. These variables are father's and mother's schooling, parental educational support at age 12, whether or not having a higher educated partner and partner's age. In order to avoid too many drop outs in the earnings regressions, we replaced the missing information by the population mean and included a dummy in the regressions indicating the missing value. This has no effect on the other coefficients and yields similar results as running the models without this procedure.
} 
4 Education, cognitive skills, and earnings of males and females

Table 4.5: Estimated coefficients from log earning regressions, specified by educational level, males

\begin{tabular}{|c|c|c|c|c|c|c|c|c|c|}
\hline & \multicolumn{9}{|c|}{ Males } \\
\hline & \multirow{2}{*}{$\begin{array}{l}\text { All males } \\
(0)\end{array}$} & \multirow[b]{2}{*}{$(1)$} & \multirow[b]{2}{*}{$(2)$} & \multicolumn{2}{|c|}{ Lower educated } & \multicolumn{2}{|c|}{ Medium educated } & \multicolumn{2}{|c|}{ Higher educated } \\
\hline & & & & $(1)$ & $(2)$ & $(1)$ & $(2)$ & $(1)$ & $(2)$ \\
\hline \multirow[t]{2}{*}{ Math score } & $0.069 * * *$ & $0.029 * * *$ & $0.027 * * *$ & $0.032 * * *$ & $0.032 * * *$ & 0.014 & 0.014 & 0.011 & 0.012 \\
\hline & $(0.006)$ & $(0.006)$ & $(0.006)$ & $(0.008)$ & $(0.008)$ & $(0.010)$ & $(0.010)$ & $(0.014)$ & $(0.014)$ \\
\hline \multirow[t]{2}{*}{ Language score } & $0.037 * * *$ & 0.003 & 0.002 & 0.003 & 0.002 & -0.002 & 0.003 & -0.012 & -0.015 \\
\hline & $(0.006)$ & $(0.006)$ & $(0.006)$ & $(0.007)$ & $(0.007)$ & $(0.010)$ & $(0.009)$ & $(0.015)$ & $(0.015)$ \\
\hline \multirow[t]{2}{*}{ IQ score } & $0.012 * *$ & 0.006 & 0.005 & 0.008 & 0.008 & 0.004 & 0.003 & -0.001 & -0.002 \\
\hline & $(0.005)$ & $(0.005)$ & $(0.005)$ & $(0.007)$ & $(0.007)$ & $(0.009)$ & $(0.008)$ & $(0.012)$ & $(0.011)$ \\
\hline \multirow[t]{2}{*}{ Years of schooling } & & $0.036 * * *$ & $0.0032 * * *$ & - & - & - & - & - & - \\
\hline & & $(0.002)$ & $(0.002)$ & & & & & & \\
\hline \multicolumn{10}{|l|}{ Field of study: } \\
\hline \multirow[t]{2}{*}{ - Agriculture } & & $-0.102 * * *$ & $-0.104 * * *$ & -0.066 & $-0.082 *$ & -0.092 & -0.074 & $0.099 * *$ & $0.097 * *$ \\
\hline & & $(0.019)$ & $(0.019)$ & $(0.044)$ & $(0.041)$ & $(0.068)$ & $(0.071)$ & $(0.046)$ & $(0.045)$ \\
\hline \multirow[t]{2}{*}{ - Economics } & & Ref. & Ref. & -0.060 & -0.058 & -0.018 & -0.008 & $0.244^{* * *}$ & $0.246 * * *$ \\
\hline & & & & $(0.050)$ & $(0.048)$ & $(0.073)$ & $(0.075)$ & $(0.036)$ & $(0.035)$ \\
\hline \multirow[t]{2}{*}{ - Engineering } & & $-0.054 * * *$ & $-0.053 * * *$ & -0.056 & $-0.067 *$ & -0.041 & -0.023 & $0.150 * * *$ & $0.151^{* * *}$ \\
\hline & & $(0.014)$ & $(0.013)$ & $(0.043)$ & $(0.068)$ & $(0.072)$ & $(0.075)$ & $(0.035)$ & $(0.035)$ \\
\hline \multirow[t]{2}{*}{ - Health } & & $-0.063 * *$ & $-0.077 * *$ & -0.016 & -0.017 & -0.043 & -0.028 & $0.125^{* *}$ & 0.098* \\
\hline & & $(0.031)$ & $(0.030)$ & $(0.078)$ & $(0.077)$ & $(0.084)$ & $(0.087)$ & $(0.052)$ & $(0.051)$ \\
\hline \multirow[t]{2}{*}{ - Other } & & $-0.128 * * *$ & $-0.126 * * *$ & - & - & - & - & Ref. & Ref. \\
\hline & & $(0.022)$ & $(0.022)$ & & & & & & \\
\hline \multirow[t]{2}{*}{ - General } & & $0.029 *$ & 0.022 & Ref. & Ref. & Ref. & Ref. & - & - \\
\hline & & $(0.016)$ & $(0.016)$ & & & & & & \\
\hline Controls for school sub levels ${ }^{\mathrm{a}}$ & - & - & - & Yes & Yes & Yes & Yes & Yes & Yes \\
\hline $\begin{array}{l}\text { Controls for social background and } \\
\text { current family composition }\end{array}$ & No & No & Yes & No & Yes & No & Yes & No & Yes \\
\hline Constant & $\begin{array}{l}7.988 * * * \\
(0.005)\end{array}$ & $\begin{array}{l}7.559 * * * \\
(0.029)\end{array}$ & $\begin{array}{l}6.898 * * * \\
(0.157)\end{array}$ & $\begin{array}{l}7.910 * * * \\
(0.016)\end{array}$ & $\begin{array}{l}7.020 * * * \\
(0.210)\end{array}$ & $\begin{array}{l}8.033^{* * *} \\
(0.020)\end{array}$ & $\begin{array}{l}7.064 * * * \\
(0.368)\end{array}$ & $\begin{array}{l}8.003 * * * \\
(0.031)\end{array}$ & $\begin{array}{l}6.890 * * * \\
(0.236)\end{array}$ \\
\hline $\mathrm{N}$ & 5757 & 5757 & 5757 & 2739 & 2739 & 1742 & 1742 & 1276 & 1276 \\
\hline Adjusted $\mathrm{R}^{2}$ & 0.072 & 0.152 & 0.192 & 0.018 & 0.058 & 0.049 & 0.110 & 0.115 & 0.171 \\
\hline
\end{tabular}

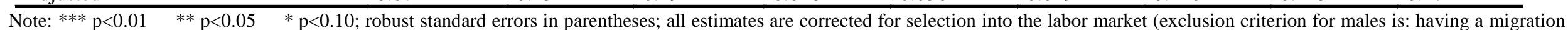

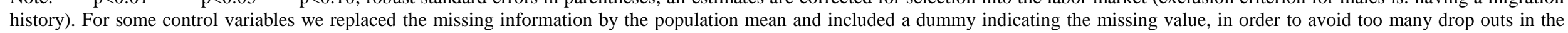
regression. These variables are: father's and mother's schooling, parental educational support at age 12, having a higher educated partner, partner's age and partner's age squared.

a) Within the low, medium and high education levels there are some minor differences in sublevels (e.g. as between BA and MA/MSc for higher educated students) for which we control.

b) Controls for social background include father's and mother's schooling the amount of parental educational support at age 12 and whether or not having one or more siblings. Controls for family

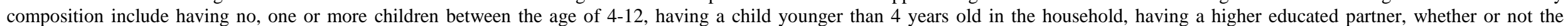
(existing) partner has any kind of income, partner's age and partner's age squared. 
4 Education, cognitive skills, and earnings of males and females

Table 4.6: Estimated coefficients from log earning regressions, specified by educational level, females

\begin{tabular}{|c|c|c|c|c|c|c|c|c|c|}
\hline \multirow[b]{3}{*}{ Variable } & \multirow{3}{*}{$\begin{array}{l}\text { All females } \\
(0)\end{array}$} & \multirow[b]{3}{*}{$(1)$} & \multirow[b]{3}{*}{$(2)$} & \multirow{2}{*}{\multicolumn{2}{|c|}{$\begin{array}{l}\text { Females } \\
\text { Lower educated }\end{array}$}} & \multirow{2}{*}{\multicolumn{2}{|c|}{ Medium educated }} & \multirow{2}{*}{\multicolumn{2}{|c|}{ Higher educated }} \\
\hline & & & & \multirow{2}{*}{\multicolumn{2}{|c|}{$\begin{array}{l}\text { Lower educated } \\
\text { (1) }\end{array}$}} & & & & \\
\hline & & & & & & \multicolumn{2}{|c|}{ (1) $\quad(2)$} & \multicolumn{2}{|c|}{$(1) \quad(2)$} \\
\hline Math score & $\begin{array}{l}0.080 * * * \\
(0.007)\end{array}$ & $\begin{array}{l}0.037 * * * \\
(0.007)\end{array}$ & $\begin{array}{l}0.036 * * * \\
(0.007)\end{array}$ & $\begin{array}{l}0.023^{*} \\
(0.013)\end{array}$ & $\begin{array}{l}0.027^{* *} \\
(0.012)\end{array}$ & $\begin{array}{l}0.026^{* *} \\
(0.010)\end{array}$ & $\begin{array}{l}0.028 * * \\
(0.010)\end{array}$ & $\begin{array}{l}0.038 * * * \\
(0.013)\end{array}$ & $\begin{array}{l}0.040^{* * *} \\
(0.013)\end{array}$ \\
\hline Language score & $\begin{array}{l}0.059 * * * \\
(0.007)\end{array}$ & $\begin{array}{l}0.026 * * * \\
(0.007)\end{array}$ & $\begin{array}{l}0.019 * * \\
(0.007)\end{array}$ & $\begin{array}{l}0.048^{* * *} \\
(0.012)\end{array}$ & $\begin{array}{l}0.040^{* * *} \\
(0.012)\end{array}$ & $\begin{array}{l}0.003 \\
(0.010)\end{array}$ & $\begin{array}{l}0.002 \\
(0.010)\end{array}$ & $\begin{array}{l}-0.009 \\
(0.013)\end{array}$ & $\begin{array}{l}-0.010 \\
(0.013)\end{array}$ \\
\hline IQ score & $\begin{array}{l}0.023 * * * \\
(0.006)\end{array}$ & $\begin{array}{l}0.012^{* *} \\
(0.006)\end{array}$ & $\begin{array}{l}0.011^{*} \\
(0.006)\end{array}$ & $\begin{array}{l}0.015 \\
(0.011)\end{array}$ & $\begin{array}{l}0.015 \\
(0.011)\end{array}$ & $\begin{array}{l}-0.001 \\
(0.009)\end{array}$ & $\begin{array}{l}-0.001 \\
(0.009)\end{array}$ & $\begin{array}{l}0.021^{*} \\
(0.011)\end{array}$ & $\begin{array}{l}0.016 \\
(0.011)\end{array}$ \\
\hline Years of schooling & & $\begin{array}{l}0.045^{* * *} \\
(0.003)\end{array}$ & $\begin{array}{l}0.037 * * * \\
(0.003)\end{array}$ & - & - & - & - & - & - \\
\hline \multicolumn{10}{|l|}{ Field of study: } \\
\hline - Agriculture & & $\begin{array}{l}-0.077 * * \\
(0.035)\end{array}$ & $\begin{array}{l}-0.095^{* * *} \\
(0.035)\end{array}$ & $\begin{array}{l}0.055 \\
(0.082)\end{array}$ & $\begin{array}{l}0.044 \\
(0.084)\end{array}$ & $\begin{array}{l}0.132 \\
(0.172)\end{array}$ & $\begin{array}{l}0.163 \\
(0.189)\end{array}$ & $\begin{array}{l}-0.020 \\
(0.053)\end{array}$ & $\begin{array}{l}-0.053 \\
(0.053)\end{array}$ \\
\hline - Economics & & Ref. & Ref. & $\begin{array}{l}0.109 \\
(0.079)\end{array}$ & $\begin{array}{l}0.115 \\
(0.080)\end{array}$ & $\begin{array}{l}0.172 \\
(0.153)\end{array}$ & $\begin{array}{l}0.229 \\
(0.171)\end{array}$ & $\begin{array}{l}0.194 * * * \\
(0.025)\end{array}$ & $\begin{array}{l}0.174 * * * \\
(0.026)\end{array}$ \\
\hline - Engineering & & $\begin{array}{l}-0.010 \\
(0.025)\end{array}$ & $\begin{array}{l}-0.017 \\
(0.024)\end{array}$ & $\begin{array}{l}0.057 \\
(0.077)\end{array}$ & $\begin{array}{l}0.069 \\
(0.078)\end{array}$ & $\begin{array}{l}0.203 \\
(0.148)\end{array}$ & $\begin{array}{l}0.252 \\
(0.168)\end{array}$ & $\begin{array}{l}0.104 * * * \\
(0.036)\end{array}$ & $\begin{array}{l}0.086^{* *} \\
(0.036)\end{array}$ \\
\hline - Health & & $\begin{array}{l}-0.088^{* * *} \\
(0.017)\end{array}$ & $\begin{array}{l}-0.063^{* * *} \\
(0.017)\end{array}$ & $\begin{array}{l}0.028 \\
(0.074)\end{array}$ & $\begin{array}{l}0.049 \\
(0.075)\end{array}$ & $\begin{array}{l}0.121 \\
(0.153)\end{array}$ & $\begin{array}{l}0.201 \\
(0.171)\end{array}$ & $\begin{array}{l}0.050 * \\
(0.027)\end{array}$ & $\begin{array}{l}0.047^{*} \\
(0.027)\end{array}$ \\
\hline - Other & & $\begin{array}{l}-0.079 * * * \\
(0.019)\end{array}$ & $\begin{array}{l}-0.075^{* * *} \\
(0.019)\end{array}$ & & & - & - & Ref. & Ref. \\
\hline - General & & $\begin{array}{l}0.090 * * * \\
(0.018)\end{array}$ & $\begin{array}{l}0.074 * * * \\
(0.018)\end{array}$ & Ref. & Ref. & Ref. & Ref. & - & - \\
\hline Controls for school sub levels ${ }^{\mathrm{a}}$ & - & - & - & Yes & Yes & Yes & Yes & Yes & Yes \\
\hline $\begin{array}{l}\text { Controls for social background and } \\
\text { current family composition }{ }^{\mathrm{b}}\end{array}$ & No & No & Yes & No & Yes & No & Yes & No & Yes \\
\hline Constant & $\begin{array}{l}7.789 * * * \\
(0.006)\end{array}$ & $\begin{array}{l}7.205^{* * *} \\
(0.039)\end{array}$ & $\begin{array}{l}7.341^{* * *} \\
(0.220)\end{array}$ & $\begin{array}{l}7.696 * * * \\
(0.024)\end{array}$ & $\begin{array}{l}8.129 * * * \\
(0.408)\end{array}$ & $\begin{array}{l}7.881^{* * *} \\
(0.018)\end{array}$ & $\begin{array}{l}7.898 * * * \\
(0.368)\end{array}$ & $\begin{array}{l}7.889 * * * \\
(0.020)\end{array}$ & $\begin{array}{l}7.492^{* * *} \\
(0.414)\end{array}$ \\
\hline $\mathrm{N}$ & 5168 & 5168 & 5168 & 1989 & 1989 & 2136 & 2136 & 1043 & 1043 \\
\hline Adjusted $\mathrm{R}^{2}$ & 0.091 & 0.164 & 0.202 & 0.056 & 0.097 & 0.069 & 0.115 & 0.154 & 0.181 \\
\hline
\end{tabular}

Note: *** $\mathrm{p}<0.01 \quad * * \mathrm{p}<0.05 \quad * \mathrm{p}<0.10$; robust standard errors in parentheses; all estimates are corrected for selection into the labor market (exclusion criteria for females are: having a child

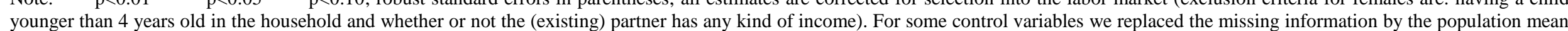

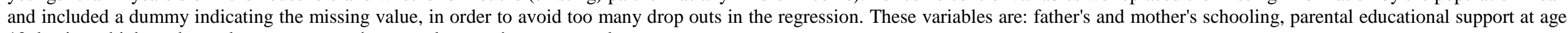
12, having a higher educated partner, partner's age and partner's age squared.

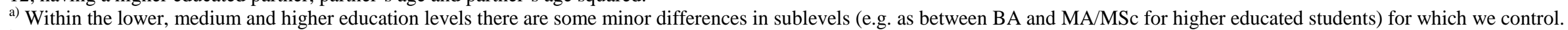

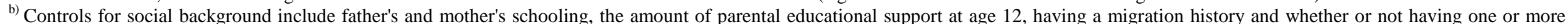
siblings. Controls for family composition include having no, one or more children between the age of 4-12, having a higher educated partner (if existent), partner's age and partner's age squared. 
As indicated earlier, the first earnings equation assumes that returns to cognitive skills are the same for each level of schooling. That is a strong assumption. Schooling is crucial to improve an individual's labor market outcome and usually sorts people into different labor markets and occupations. We may also expect that skills are differently rewarded in these labor markets, with some skills being specifically rewarded in high level jobs. At the same time, cognitive skills can serve as a substitute for schooling, thus leading to higher returns for the lower educated. Interestingly, the results for the second equation, which specifies the model by level of education, shows that in the case of males this effect of math skills on earnings is only significant for lower educated males, while for females the effect of math skills remains significant for all levels of education and is even highest for the higher educated females (4\% wage premium for a one standard deviation increase in the math skills, compared to only $1.3 \%$ for males).

For lower educated females we also note significant earnings effects of language skills which are absent in the case of males of all specifications. ${ }^{53}$ For those women the wage premium of language skills is even higher than of math skills.

Tables 4.5 and 4.6 also show how earnings are affected by study field choices. For lower and medium educated males and females the study field differences are rather small, but for higher educated the study field is an important determinant of earnings. Economics studies ${ }^{54}$ are by far the most lucrative study field in higher education. For males the earnings advantage is $10 \%$ compared to engineering, $15 \%$ compared to agriculture and health and $25 \%$ compared to other studies. For females the wage premium of having followed a study in economics in higher education is about $9 \%$ compared to engineering, about $13 \%$ compared to health and about $18 \%$ compared to 'other studies'. To examine whether cognitive skills (here, specifically math skills) have a 'residual impact' on earnings after correcting for education, we run the earnings regressions with interaction terms between math skills and each study field, separately for males and females. For females, we indeed find a residual impact of math skills on earnings differing per study field. This impact is significant for lower and medium educated females choosing agricultural studies compared to general studies, and for higher

\footnotetext{
53 The effect of math skills on males' and females' log earnings does not explain much of the gender wage gap. As indicated in Table 4.1, males' average earnings are 20\% higher than those of females. Including only math skills in the males' and females' earnings regression reduces the males' earnings advantage by some $2 \%$, while controlling either for language skills or non-verbal intelligence the males' earnings advantage slightly increases again (analyses are available on request).

${ }^{54}$ This is the reference category and includes also business studies.
} 
educated females choosing economics studies compared to other studies. For males, we do not find significant effects. ${ }^{55}$

Figures 4.4a-4.4c show the effect of cognitive skills on earnings graphically. This model includes a quadratic effect of cognitive skills on earnings to allow for non-linearity. ${ }^{56}$ We can draw some interesting conclusions from this. All three figures show the familiar pattern of higher earnings for males than for females. Figure 4.4a shows that for females the returns to math skills are almost linear, but for males the returns to low math skills are relatively high. We will return to that issue below. Figure $4.4 \mathrm{~b}$ shows that the returns to language skills are linear for both males and females, but the effect is stronger for females (albeit not as strong as for math skills). Figure 4.4c shows a small linear effect of IQ on earnings for females, and weak effect for males that clearly tapers off at the high end of the scale.

Figure 4.4a: Log earnings at math scores, controlled for math squared, males and females

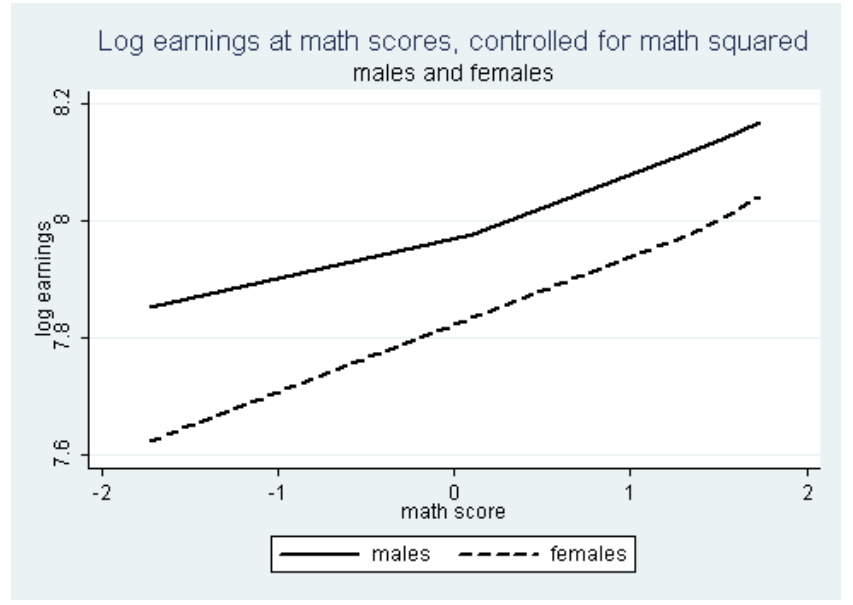

\footnotetext{
${ }^{55}$ The results are available on request.

${ }^{56}$ In these models we control only for cognitive skills and their quadratic effect. Full results of the analyses are available on request.
} 
Figure 4.4b: Log earnings at language scores, controlled for language squared, males and females

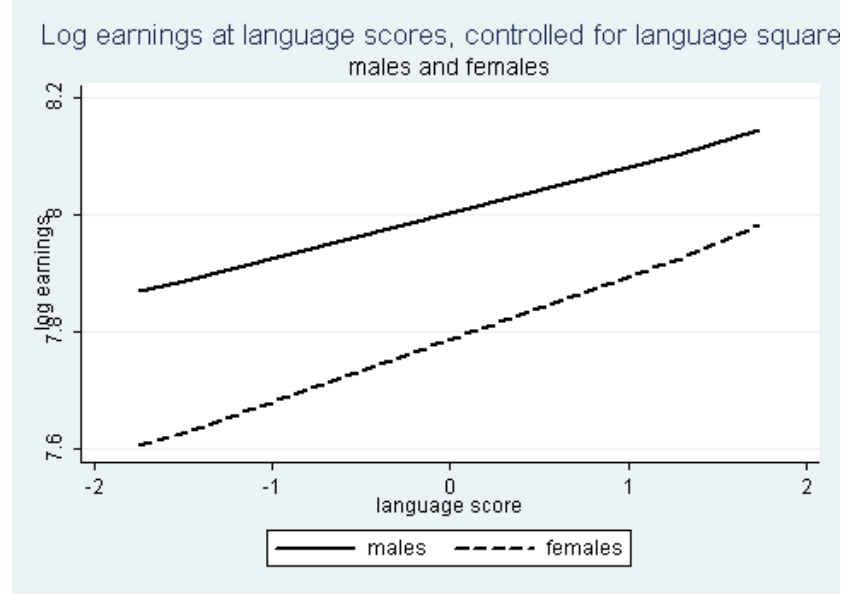

Figure 4.4c: Log earnings at IQ scores, controlled for IQ squared, males and females

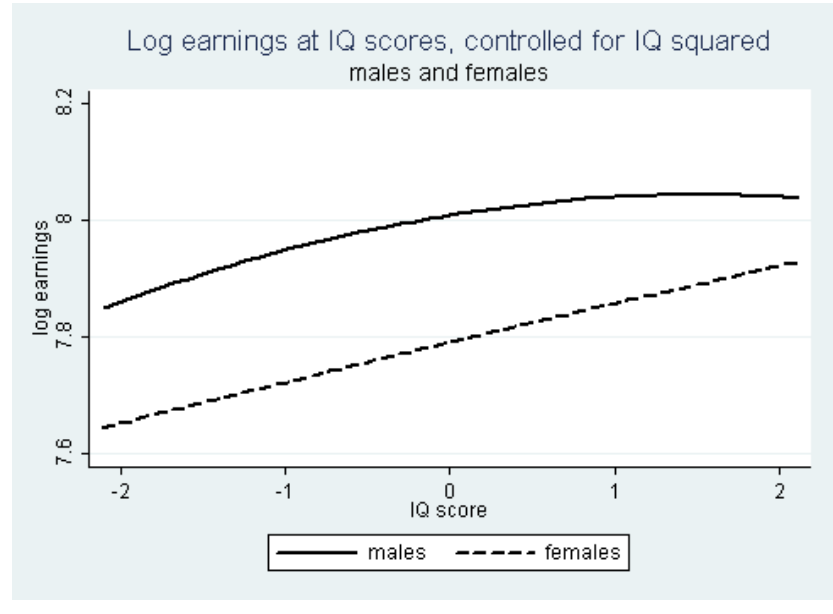

How can these gender differences in the returns to cognitive skills be explained? In Table 4.6 all these estimates are controlled for selection effects of female's study field choices and labor supply decisions. We performed additional analyses with controls for job sector and part time work, ${ }^{57}$ but this does not change the substantive results.

There is no a priori reason to think that employers value math skills in similar jobs differently for males and females. One reason why we might find these results for females and not for males could be related to a differential (unobserved) sorting process in school. As the residual impact of math skills on earnings per study field is also significant, one

\footnotetext{
${ }^{57}$ We additionally run models which include controls for job sector as well as models with interaction terms for math skills and a part time-dummy, and for math skills and a public sector-dummy, assuming that working parttime or in a public sector is negatively associated with math skills. The results from Tables 4.3 and 4.4 on the returns to cognitive skills are robust to these tests. The estimates of the two interaction terms are not significant nor did they change the magnitude of the math skills-coefficient substantially.
} 
explanation can be that females on average select in study fields that do not fully match their specific talents. However, their 'additional skills' are rewarded on the labor market, as they probably exceed the required qualification on the job.

\subsection{Conclusion}

Recent studies on returns to cognitive skills vary in their results regarding males' and females' labor market outcomes. This chapter analyzed earnings returns to different cognitive skills measures directly and indirectly, through study field choices and the attained educational level. We used a unique data set combining longitudinal data of the Secondary Education Pupils Cohort (SLVO) and earnings information from national tax files from Statistics Netherlands. We estimated the effect of math and language skills, as well as IQ, all measured at the age of 12 , on earnings at age 35, controlling for social background, educational level, study field, job and partner characteristics and the family situation at the time when earnings are observed.

Our findings show that cognitive skills and specifically math skills are rewarded on the labor market, but more for females than for males, in general and conditional on education. Females also get a wage premium for language skills and IQ, but to a lesser extent than from math skills. Specifying the earning regressions by educational level indicates that math skills only affect the earnings of lower educated males, while for females the effects are significant at all levels, but strongest for the higher educated.

We argue that the process through which cognitive skills are converted into schooling outcomes (both years of schooling and study field) is different for males and females. For males, the effect of cognitive skills is largely indirect, affecting their educational level and study field choices more strongly than for females. For females on the other hand, we note that cognitive skills do not fully convert into years of schooling or more 'appropriate' study field choices. The predictive power of cognitive skills on years of schooling is $18 \%$ for females, compared to $24 \%$ for males. Although gender differences in years of schooling are rather small and insignificant, females' selection in education appears to be less based on their cognitive skills. It suggests that part of females' success in achieving higher educational levels must be attributed to other factors, such as motivation or diligence. The literature in this field indeed points to such characteristics that help explaining the effect (e. g. Francis, 2006; Buchmann, DiPrete, and McDaniel, 2008). It furthermore suggests that females' educational success could be even stronger if selection would have been based more on their 
4 Education, cognitive skills, and earnings of males and females

cognitive skills, as it is in the case of males. We thus conclude that the meritocratic process in education works better for males than for females. Once males have achieved higher educational levels, they are also more likely to choose programs with high earning perspectives like engineering, even if their math skills level is relatively low. For females, it appears there is still a 'reserve of talent' that is not fully 'recognized' in the education system, but that is nevertheless rewarded on the labor market. 
5 How education, cognitive skills, and cultural and social capital account for intergenerational earnings persistence 
5 How education, cognitive skills, and cultural and social capital account for intergenerational earnings persistence

\subsection{Introduction}

Intergenerational income mobility is defined as a child's potential to move up (or down) the income scale relative to the child's parents (Organisation for Economic Cooperation and Development, OECD, 2010). ${ }^{1}$ This is usually estimated as the elasticity between a father's and son's earnings. ${ }^{2}$ Most of this intergenerational income mobility is assumed to be transmitted through the effects of a father's earnings on his son's educational attainment, which in turn affects the son's own earnings. Other transmission mechanisms can involve cognitive and non-cognitive skills transmitted from one generation to the next. Unequal distributions of biological and social endowments and inequality in families' financial potential to invest in their children's educational and labor market careers produce unequal opportunities across different social groups.

Moreover, countries differ in the extent to which these unequal opportunities are strengthened or weakened. Some education systems are more prone to increasing social inequality than others (Shavit and Blossfeld, 1993; Hanushek and Woessmann, 2006). As a result, we find large differences in earnings elasticity across countries, ranging from a low of 0.15 in Denmark to a high of 0.50 for the United Kingdom (Corak, 2006). A recent study by Moonen and Van den Brakel (2011) shows an estimate for the Netherlands of $0.22,{ }^{3}$ which would make the Netherlands one of a group of countries with relatively high mobility, between Canada (0.19) and Sweden (0.27) (Corak, 2006).

The main aim of this study is to examine the relative importance of transmission mechanisms that are directly or indirectly related to the association between a father's and his son's earnings. This strand is less often followed in the economic literature. ${ }^{4}$ Sociological studies are more often interested in these transmission mechanisms, but focus on the intergenerational mobility of socioeconomic status (SES) rather than of earnings. We attempt to combine both strands of research. We apply the conditional earnings mobility approach suggested by Blanden et al. (2007) and include-besides education and cognitive skills-

\footnotetext{
${ }^{1}$ This chapter is based on joint work with Frank Cörvers, Tanja Traag, and Rolf van der Velden.

${ }^{2}$ The intergenerational income elasticity directly translates the ratio into the economic advantage a child from the higher income family can expect to have in the next generation over one from the lower income family. A lower elasticity translates into smaller advantages for children from higher income family (Corak, 2006).

${ }^{3}$ Earlier estimates for the Netherlands were made by Cörvers (2000a, 2000b, 2004) and Van den Brakel and Moonen (2009).

${ }^{4}$ Most studies in the economic literature concentrate on measurement problems in estimating earnings elasticities, for instance, trying to reduce lifecycle bias and measurement error in estimating lifetime income with current income information (e.g., Jenkins, 1987; Grawe, 2006; Haider and Solon, 2006; Nybom and Stuhler, 2011).
} 
5 How education, cognitive skills, and cultural and social capital account for intergenerational earnings persistence

measures of the son's cultural and social capital. The transmission mechanisms of both cultural and social capital are new in the intergenerational income mobility literature. We estimate the extent to which these transmission mechanisms account for overall intergenerational earnings elasticity in the Netherlands. To do so, we exploit a unique and rich data set that combines panel data from a birth cohort with earnings data from national tax files and a large nationwide income survey.

From a country-specific perspective, the Netherlands is interesting to study since they are seen as a "knowledge-based society," where education is considered the main factor explaining social and economic outcomes, rather than a "class society," such as in the United Kingdom (Kraaykamp et al., 2010). This would suggest that educational attainment and cognitive skills are the main transmission mechanism. ${ }^{5}$ However, as indicated above, other transmission mechanisms, such as cultural and social capital can also play an important role in determining intergenerational income persistence. As in the study of Blanden et al. (2007) for the United Kingdom, we decompose the intergenerational earnings elasticity for the Netherlands into several mediating factors that may account for the transmission of earnings between fathers and sons. As in the UK study, we consider son's cognitive skills (measured by a math performance test, a language performance test, and an IQ test at age 12) and educational attainment (measured by years of schooling, field of study, and whether or not classes have been repeated during primary education) as transmission mechanisms. Furthermore, we include in our analysis measures for the cultural capital of the parents (measured by the number of books the parents read and whether or not they regularly visit museums, concerts, or theater plays) and the social capital of the neighborhood (measured by the socioeconomic composition of the neighborhood in which the sons grew up). Ioannides and Datcher Loury (2004) extensively discuss the relevance of neighborhood effects for climbing up the social and income ladder. As an additional test of our model, we replace a son's earnings with his house property value, considering this variable a proxy for his permanent life income.

Our findings show that the son's cognitive skills and schooling account for about $50 \%$ of the association between his and his father's earnings. By including the cultural capital of the parents and the social capital of the neighborhood, the explained part increases by $6 \%$.

\footnotetext{
${ }^{5}$ Blanden et al. (2007) state that transmission mechanisms, such as education, ability, noncognitive skills, and labor market experience have returns in the labor market and are linked with permanent incomes of parents and children across generations. Grawe (2008) points out that families with higher earnings and those with higher wealth stocks are better able to finance the education and occupational start-up costs (e.g., for self-employment) of their children, which, in turn, reinforces financial advantages across generations.
} 
5 How education, cognitive skills, and cultural and social capital account for intergenerational earnings persistence

That is, all our mechanisms together account for $56 \%$ of the intergenerational earnings persistence. This means that $44 \%$ of the intergenerational earnings persistence is related to other, unobserved factors. Regarding the specific contribution of each transmission mechanism, education accounts for the largest part, while cognitive skills mainly work indirectly through educational attainment. This is followed by the effect of social capital, which appears to play a bigger role than the parents' cultural capital in explaining intergenerational earnings persistence.

The remainder of this chapter is organized as follows. Section 5.2 introduces the modeling approach and decomposition procedure adapted from the work of Blanden et al. (2007). Section 5.3 describes the data and defines the sample for empirical analysis. Section 5.4 presents the results regarding earnings elasticity estimates and the mechanisms that account for intergenerational persistence. Section 5.5 summarizes the study and Section 5.6 discusses the findings with regard to future research.

\subsection{Modeling approach and estimation procedure}

Due to the lower labor market participation rate of women, especially in older cohorts, most of the literature on intergenerational mobility concentrates on the earnings of fathers and sons. ${ }^{6}$ As in other studies, we estimate intergenerational mobility by regressing the logarithm of the son's earnings on the logarithm of the father's earnings:

$$
\ln Y_{i}^{\text {son }}=\alpha_{0}+\beta_{1} \ln Y_{i}^{\text {father }}+\varepsilon_{i}
$$

where $i$ indicates the family to which the father and son belong, $\beta_{1}$ is the elasticity ${ }^{7}$ of the son's $\log$ earnings, $\ln Y_{i}^{\text {son }}$, with regard to the father's $\log$ earnings, $\ln Y_{i}^{\text {father }}$, and $\varepsilon_{i}$ is an idiosyncratic error term.

One issue to consider is transitory fluctuations due to the income of fathers and sons being measured at different ages. Following Solon (1992) and Corak and Heisz (1999), we correct for lifecycle differences by including father's age and age squared ${ }^{8}$.

\footnotetext{
${ }^{6}$ For estimates on the father-daughter relationship, see Moonen and Van den Brakel (2011).

${ }^{7}$ Empirical studies in rich countries find $\beta$ to lie between zero and one. For example, a value of one would indicate complete intergenerational persistence of incomes and a value of zero complete intergenerational mobility (Corak, 2006).

${ }^{8}$ For sons this correction makes no sense in our analysis, since they all come from the same birth cohort.
} 
5 How education, cognitive skills, and cultural and social capital account for intergenerational earnings persistence

$$
\ln Y_{i}^{\text {son }}=\alpha_{0}+\beta_{1} \ln Y_{i}^{\text {father }}+\beta_{2} \text { FatherAge }+\beta_{3} \text { FatherAge }^{2}+\varepsilon_{i}
$$

The lifecycle differences also partly reflect the measurement issue of current versus permanent income. The data allow us to measure income for only a single year for fathers aged between 38 and 58 and for sons around the age of 31. Measuring mobility based on single-year earnings information is likely to underestimate the true beta. ${ }^{9}$ As outlined by Blanden (2009), age-earnings profiles are steeper for those with a higher permanent income. Furthermore, "at young ages current income is low compared to permanent income for those with high permanent income, while at older ages current income is higher compared to permanent income for those with high permanent income” (Blanden, 2009: 5). Permanent income that averages income over several years is therefore to be preferred to current income (Solon, 1992; Corak and Heisz, 1999; Haider and Solon, 2006). ${ }^{10}$

In the case of the father's earnings, this is probably less of an issue. Since the earnings are gathered around the age of 45, they give a good approximation for lifetime earnings, since labor market careers are usually settled at this age (Grawe, 2003, 2004; Corak, 2006). Having single-year earnings information in the case of the sons is probably more problematic, because of the aforementioned steeper age-earnings profiles for those with a high permanent income. This study therefore carries out an additional test by looking at the son's house property value as an alternative proxy for his permanent income. This house property value was also measured in 2008, when the sons were about 31 years old. Furthermore, we compare our earnings elasticity estimate with that reported by Moonen and Van den Brakel (2011), who used another data set for the Netherlands.

As indicated in the introduction, sociological studies have dealt with the same subject, but focused more on intergenerational status mobility rather than intergenerational income mobility (Blau and Duncan, 1967; Bourdieu, 1973; Boudon, 1974). The classic status attainment model assumes that the primary mechanism underlying this relation is that the father's SES affects both his offspring's cognitive skills as well as the choices they make in

\footnotetext{
${ }^{9}$ An alternative way to measure the father's permanent income is to instrument his income, usually by using his education, occupation, or social class as instrumental variables (Björklund and Jäntti, 1997; Dearden, Machin, and Reed, 1997). However, it is questionable whether these are good instruments, since they not only are correlated with the endogenous variable (the father's earnings), but also directly affect the son's earnings (Solon, 1992, 2002; Corak, 2006).

${ }^{10}$ Corak and Heisz (1999) suggest averaging the father's income over a period of at least five consecutive years to reduce the influence of transitory variations. Mazunder (2005) suggests averaging over even more years, since a five-year period is still too short to overcome measurement error and to be truly representative of lifecycle income.
} 
5 How education, cognitive skills, and cultural and social capital account for intergenerational earnings persistence

education (the primary and secondary effects of social stratification, see Boudon, 1974). Both effects determine the son's level of educational attainment, which in turn determines his own SES. There is also a weaker direct effect from the father's SES on the son's SES, indicating some direct inheritance.

The main transmission mechanisms put forward in the sociological literature to explain family effects on a son's educational and occupational attainment are the different forms of capital available to the family: economic, cultural, and social capital. Economic capital is directly linked to a father's earnings and reflects the possibilities of the family investing in their children, for instance, buying books or sending them to good schools. The term cultural capital was introduced by Bourdieu (1973) and refers to the symbols, ideas, tastes, and preferences that can be used as resources in social interaction. Typically, the cultural capital of middle-class parents endows their children with the linguistic and cultural competence needed to be successful in school. This study measures the concept of cultural capital by the number of books the parents read and whether or not they regularly visit museums, concerts, or theater plays. These proxies have been shown to be strong predictors of educational attainment (Georg, 2004).

The concept of social capital was originally developed by Coleman and Hoffer (1987). It refers to a person's relations with the broader community. As shown by the authors, social capital can have a positive impact on a person's development in providing a set of norms, especially in communities with high closure. In our study a family’s social capital is proxied by the socioeconomic composition of the neighborhood that is derived from the definition and the computation of the Netherland's Institute for Social Research (Sociaal en Cultureel Planbureau (SCP) 1998). ${ }^{11}$ The neighborhood is defined as the postal code area in which the son lived at age 15. Postal code areas are neighborhoods within cities or municipalities. The Netherlands counts almost 4,000 different postal code areas. The socioeconomic composition of each neighborhood is based on principal component analysis that includes variables on that neighborhood's average household income, share of lowincome households, share of people without a paid job, and share of households that, on average, have a low education. ${ }^{12}$ We assume that the social capital of the neighborhood

\footnotetext{
${ }^{11}$ Many studies on social capital also use the marital status of the parents and indicate that single parents have less social capital than couples (Pong and Ju, 2000). Since single parents are often female, this is not a decent indicator for the current analyses, since we are specifically interested in father-son earnings elasticity.

12 The analysis yields a first unrotated component that explains $65 \%$ of the total variance. The factor loadings are as follows: average household income, 0.88; share of low-income households, 0.83; share of people without a paid job, 0.71; and share of households that, on average, have a low education, 0.78 (Sociaal en Cultureel Planbureau, 1998). The social status score is standardized with mean zero and standard deviation one. The
} 
5 How education, cognitive skills, and cultural and social capital account for intergenerational earnings persistence

affects values and norms such as academic interest and adherence to school norms. Moreover, the social capital of the neighborhood can provide a network for the son that can improve his job market opportunities and thus earnings potential. ${ }^{13}$

With regard to the methodological setup, we adopt the conditional earnings mobility approach of Blanden et al. (2007) to account for the relative contribution of four different mediating factors in the intergenerational earnings relationship. We assume that a son's earnings are a function of his cognitive skills and schooling, his parents' cultural capital, and the social capital of the neighborhood:

$$
\ln Y_{i}^{\text {son }}=\lambda+\pi \text { CognSkills }_{i}^{\text {son }}+\rho \text { Educ }_{i}^{\text {son }}+v_{\text {CultCap }}^{\text {parents }}+\operatorname{CSocCap}_{i}^{\text {neighbourhood }}+u_{i}
$$

where CognSkills son is a vector of several cognitive skills measures, $E d u c_{i}^{\text {son }}$ is a vector of the son's schooling measures, ${ }^{14}$ and CultCap ${ }_{i}^{\text {parents }}$ and SocCap ${ }_{i}^{\text {neighbourhood }}$ represent vectors of the parent's cultural capital and a proxy for the social capital of the neighborhood, respectively. To account for the role of these characteristics as transmission mechanisms, we estimate them separately as a function of the father's earnings:

$$
\begin{aligned}
& \text { CognSkills }_{i}^{\text {son }}=\alpha_{1}+\gamma \ln Y_{i}^{\text {father }}+\varepsilon_{1 i} \\
& \text { Educ }_{i}^{\text {son }}=\alpha_{2}+\delta \ln Y_{i}^{\text {father }}+\varepsilon_{2 i} \\
& \text { CultCap }_{i}^{\text {parents }}=\alpha_{3}+\phi \ln Y_{i}^{\text {father }}+\varepsilon_{3 i} \\
& \text { SocCap }_{i}^{\text {neighbourhood }}=\alpha_{5}+\kappa \ln Y_{i}^{\text {father }}+\varepsilon_{5 i}
\end{aligned}
$$

Equations (5.4) to (5.7) thus display the univariate relationships between the transmission mechanisms and the father's earnings. For the conditional decomposition, we multiply the returns to cognitive skills by the relationship between the father's earnings and cognitive

original scores are reversed so that a high score is associated with a high social status and a low score is associated with a low social status.

${ }^{13}$ Ioannides and Datcher Loury (2004) claim that wage premiums and wage penalties are associated with finding jobs through personal contacts.

${ }^{14}$ We measure a son's cognitive skills by performance tests in mathematics and language and an IQ test at the age of 12. The son's schooling is estimated by the number of years of schooling, the field of study in which he attained his highest educational degree, and whether or not he repeated classes during primary education. As pointed out by Heckman et al. (2006), schooling and cognitive skills are highly correlated. That is, the estimates of the effect of cognitive skills on earnings are larger when we do not control for schooling. 
5 How education, cognitive skills, and cultural and social capital account for intergenerational earnings persistence

skills and continue with the same procedure regarding the returns to other transmission mechanisms and their relationship to the father's earnings:

$$
\beta=\pi \gamma+\rho \delta+v \phi+\omega \eta+\frac{\operatorname{Cov}\left(u_{i}, \ln Y_{i}^{\text {father }}\right)}{\operatorname{Var}\left(\ln Y_{i}^{\text {father }}\right)}
$$

where $\pi \gamma$ is the conditional contribution of cognitive skills, $\rho \delta$ is the conditional contribution of schooling, and $v \varphi$ and $\omega \eta$ are the conditional contributions of cultural and social capital, respectively. ${ }^{15}$ Furthermore, a term for unexplained mobility in earnings is included that is not transmitted through these mediating factors.

\subsection{Data}

We use data from the Secondary Education Cohort of Students (VOCL 1989) matched with income information from the Dutch national tax files for the sons and with regional income survey (RIO) data, also based on tax files, for the fathers. All data were provided by Statistics Netherlands. The VOCL cohort is a nationally representative panel of children who were born in 1977 and entered secondary education for the first time in 1989, when most of them were around age $12 .{ }^{16}$ The VOCL study contains information about the parents' schooling and SES; the children's performance scores in math, language, and non-verbal intelligence at age 12; and the children's study field choices and highest educational level attained. The survey comprises 18,294 individuals, 9,460 of whom are male.

The earnings information for the sons is collected from the Dutch national tax files of 2008. After matching the cohort survey with administrative earnings data, we obtain valid earnings information for 7,718 males, which corresponds to $82 \%$ of the original sample. The earnings reflect the gross annual earnings of sons at the age of 31 . The remaining $18 \%$ of sons either are self-employed (10\%), receive social benefits (5\%), or had no income that

\footnotetext{
15 Similar to Blanden et al. (2007), we first look at the unconditional impact of each specification on intergenerational earnings persistence. We then add the schooling information to the cognitive skills information and continue with adding the cultural and social capital information to examine how the mediating factors can affect each other.

${ }^{16}$ Cohort studies have been undertaken by Statistics Netherlands on a regular basis since the mid-1970s. Other panel studies refer to cohorts born in 1965, 1970, 1981, and 1987, which all start surveying individuals at age 12. However, a reliable comparison of intergenerational earnings persistence over time with these cohorts was not possible. The main reason for this is that adequate earnings information for fathers was not available for the older cohorts and the sons were still too young in the younger cohorts to provide adequate earnings information.
} 
5 How education, cognitive skills, and cultural and social capital account for intergenerational earnings persistence

particular year (3\%). ${ }^{17}$ The earnings information of the fathers is collected from the 1995 RIO, covering a representative sample of approximately one-third of the population aged 15 years and older for which income information based on national tax files has been collected.

While matching with the administrative data of 2008 allows us to keep earnings information for all employed sons, we lose observations for fathers by matching the VOCL cohort data with the 1995 RIO data and through the matching of father-son pairs with valid earnings information. ${ }^{18}$ Again, these earnings reflect the fathers' annual gross earnings. The earnings selection criteria are the same for fathers and sons: Individuals need to have positive earnings information and had to work as an employee or civil servant for at least 51 weeks in the year earnings are observed. Further selection was made with regard to the fathers' ages, which ranged from 38 to $58 .{ }^{19}$ Conditional on these selection criteria, we obtain 2,377 fatherson pairs in our final sample.

Figure 5.1 illustrates the age-earnings profile of fathers in the selected sample. It shows that earnings rise until the age of 51 and then taper off. Table A5.1 in the appendix shows the descriptive statistics of the annual gross earnings for both fathers and sons. Regarding our transmission mechanisms variables, Table A5.2 in the appendix shows that the distributions of the explanatory variables in the selected sample do not vary much from those in the full sample, except for son's cognitive skills and the social status of his neighborhood, which are, on average, higher in the selected sample than in the full sample. ${ }^{20}$

\footnotetext{
${ }^{17}$ Social benefits refer mainly to unemployment benefits, social welfare, and disability benefits. Respondents with no income are mainly students.

${ }^{18}$ Matching the data for the fathers of the school cohort survey with the RIO data is only possible for approximately one-third of the cohort sample population. Since not all fathers obtained earnings from labor in 1995 and not every father responded to parent questionnaire in the original survey (due to either a total nonresponse or because the father was absent), the resulting proportion for which we have earnings information is even lower.

${ }^{19}$ The relatively wider age range for fathers, unlike the suggestions of Grawe (2003, 2004) and Corak (2006), was chosen to avoid losing more observations.

${ }^{20}$ For pair wise correlations between the variables of the selected sample, see Table A5.3 in the appendix.
} 
5 How education, cognitive skills, and cultural and social capital account for intergenerational earnings persistence

Figure 5.1: Father’s log earnings at father’s age

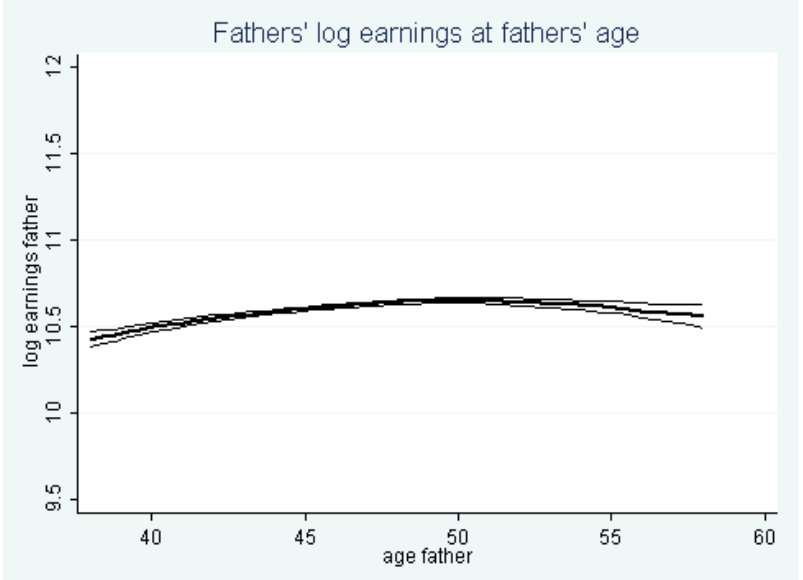

Note: Log earnings of fathers and sons are truncated at the top and bottom 1 percent.

Figure 5.2 displays the relation between a son's and his father's earnings. As expected, it shows a clear positive relation.

Figure 5.2: Sons’ log earnings at fathers’ log earnings

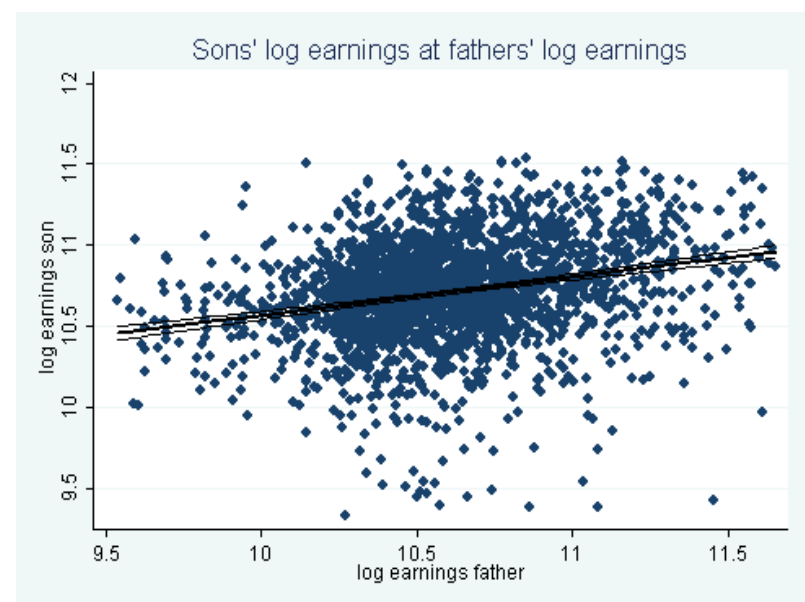

\subsection{Results}

We start with calculating the general earnings elasticity. Column 1 of Table 5.1 shows an estimate of 0.23 between fathers and sons. The partial correlation, which corrects for differences in the variance of the log earnings between generations, is not significantly different from the magnitude of the former earnings elasticity coefficient. The elasticity coefficient of column 1 replicates the estimate found by Moonen and Van den Brakel (2011). These authors apply the same selection criteria as we do here, using a relatively small sample 
5 How education, cognitive skills, and cultural and social capital account for intergenerational earnings persistence

of the Dutch population for which earnings are collected from national tax files. ${ }^{21}$ We take the fact that our coefficient is the same as theirs as an indicator that our data are representative of the Dutch population.

In column 2 of Table 5.1 we test whether outliers affect the elasticity by eliminating the top and bottom 1\% of the father's and son's earnings. This leaves 2,282 father-son pairs in the sample. The results show that the elasticity increases to 0.24 , which is not significantly different from the estimate in column 1 . For further analysis, however, we continue with the truncated sample to avoid possible follow-up biases due to outliers.

As indicated earlier, we run an additional test replacing earnings with the son's house property value in 2008. Not all sons owned a house; therefore the sample reduces further from 2,282 to 1,413 observations. ${ }^{22}$ We first check whether the earnings elasticity is affected by this selection. This is not the case; the results in column 3 of Table 5.1 show that the earnings elasticity is still 0.24 . However, the elasticity between a father's earnings and his son's house property value is much lower, only 0.09 . This is unexpectedly low, since house property value is regarded as a proxy for lifetime income. We have to consider, however, that the decade in which these sons bought their houses, 1999-2008, is characterized by an extreme increase in housing prices. The average price of houses sold then rose from $€ 145,000$ to $€ 255,000$, an increase of $75 \%$. Most of this increase occurred in the beginning of that period. The average earnings in that same period increased by $36 \%$, from $€ 21,600$ to $€ 29,400 .^{23}$ This has important consequences for a son's house property value as measured in 2008. Sons who bought their house relatively early profited much more from the rise in housing prices than sons who bought their house relatively late in that decade. ${ }^{24}$ As a result, the association between a father's earnings and his son's house property value is strongly decreased. Nevertheless, we check whether the contribution of different transmission mechanisms is more or less the same as for earnings elasticity.

\footnotetext{
${ }^{21}$ Moonen and Van den Brakel (2011) use the Income Panel Study as a database to obtain a representative sample that starts in 1985 and covers 1.5\% of the overall Dutch population. Their study examines fathers and sons of all ages and compares the 1985 earnings of fathers with the 2008 earnings of their sons.

${ }^{22}$ To test the association between a father's log of earnings and his son's log of the house property value and the relative contribution of the transmission mechanisms, we select a sample of those sons who have positive values on both earnings and house property value. As a robustness check, we run the model with the reduced sample also on the son's log of earnings.

${ }^{23}$ The estimates of the development of house prices and earnings are from Statistics Netherlands (www.statline.cbs.nl).

${ }^{24}$ Those who bought their house relatively early would typically be the lower educated sons who stopped studying at age 18-20, while those who bought relatively late would typically be higher educated sons who graduated at age 23-27.
} 
5 How education, cognitive skills, and cultural and social capital account for intergenerational earnings persistence

Table 5.1: Income elasticities

\begin{tabular}{lllllll}
\hline & {$[1]$} & & {$[2]$} & & {$[3]$} & {$[4]$} \\
\hline beta & $0.225^{* * *}(0.022)$ & $0.238^{* * *}$ & $(0.019)$ & $0.239^{* * *}(0.020)$ & $0.085^{* * *}(0.025)$ \\
partial correlation & $0.228^{* * *}(0.022)$ & $0.220^{* * *}$ & $(0.017)$ & $0.188^{* * *}(0.016)$ & $0.074 * * *(0.021)$ \\
$\mathrm{N}$ & 2377 & & 2282 & & 1413 & 1413 \\
\hline
\end{tabular}

Note: $* * * p<0.01$. Robust standard errors are in parentheses. Column [1] reports elasticity coefficients for the untruncated earnings sample, column [2] for the truncated earnings sample where the top and bottom 1\% of father's and son's earnings are removed. Column [3] reports the earnings elasticity coefficient for the truncated and selected sample (valid information on son's earnings and son's house property value). Column [4] reports the elasticity coefficient between father's log earnings and son's house property value for the truncated and selected sample (valid information on son's earnings and son's house property value). All regressions are controlled for father's age and father's age squared.

\section{Accounting for intergenerational earnings persistence}

The next step deals with the decomposition procedure, as described in Section 5.2. Column 0 of Table 5.2 displays the relation between each transmission mechanism and father's earnings. Each regression includes controls for the father's age and age squared. All mediating variables are strongly related with the father's earnings and in the expected direction. Columns 1 to 6 report the sequential earnings regressions of the relation between the chosen mediating factors and a son's earnings. ${ }^{25}$ The explanatory power of the cognitive skills variables in column 1 is almost 0.11 , while column 2 reports a higher R-squared value of 0.15 for the schooling variables. Including both specifications in column 3 increases the Rsquared value only barely, up to 0.17 .

The magnitude of the cognitive skills and schooling coefficients is reduced in column 3 , which suggests the two measures are correlated with each other in affecting earnings. With regard to the cognitive skills variables, math skills predict the strongest relation with a son's earnings. Controlled for a son's schooling, an increase of one standard deviation in math skills is associated with a 3.7\% increase in earnings. Each year of schooling also increases a son's earnings by 3.8\%, while not having repeated class during primary education increases earnings by $4.6 \%$. In addition, the chosen study field affects earnings, with positive effects for engineering, economics, and general studies (relative to health studies).

The parents' cultural capital (column 4) also has a positive effect on earnings, but this effect disappears as soon as other controls are included (column 6). The social capital of the

\footnotetext{
${ }^{25}$ To keep a maximum number of observations in these regressions, we replaced the missing values of some explanatory variables by the population mean and included dummies to indicate if a value was missing. The variables with missing values replaced are the math score, language score, IQ score, and number of books parents read. Running regressions that include only non-missing values does not lead to significant changes in the magnitude of the regression coefficients.
} 
5 How education, cognitive skills, and cultural and social capital account for intergenerational earnings persistence

neighborhood, proxied by the average SES score, has an explanatory power of 0.03 and is positively related with a son's earnings (column 5). A one standard deviation increase in the SES score of the neighborhood is associated with a $6.6 \%$ increase in a son's earnings. Column 6 includes all predictors, which raises the explanatory power to 0.18 . As could be expected, including more controls reduces the strength of most of the coefficients. The variables that remain significant in predicting a son's earnings are math skills, years of schooling, certain study fields (engineering, economics, and general studies), not having repeated classes in primary school, and the SES score of the neighborhood. 
5 How education, cognitive skills, and cultural and social capital account for intergenerational earnings persistence

Table 5.2: Relationships between transition mechanisms, father's log earnings and son’s log earnings

\begin{tabular}{|c|c|c|c|c|c|c|c|c|c|c|c|c|c|c|}
\hline Dependent variable & $\begin{array}{l}\text { Father's lo } \\
{[0]} \\
\text { coeff } \\
\end{array}$ & $\begin{array}{l}\text { earnings } \\
\mathrm{SE} \\
\end{array}$ & $\begin{array}{l}11] \\
\text { coeff } \\
\end{array}$ & SE & $\begin{array}{l}{[2]} \\
\text { coeff } \\
\end{array}$ & SE & $\begin{array}{l}{[3]} \\
\text { coeff } \\
\end{array}$ & $\begin{array}{l}\text { Son's lo } \\
\text { SE }\end{array}$ & $\begin{array}{l}\text { [4] earnings } \\
\text { coeff } \\
\end{array}$ & SE & $\begin{array}{l}{[5]} \\
\text { coeff } \\
\end{array}$ & SE & $\begin{array}{l}{[6]} \\
\text { coeff } \\
\end{array}$ & SE \\
\hline Math performance & $0.759 * * *$ & $(0.055)$ & $0.075 * * *$ & $(0.008)$ & & & $0.037 * * *$ & $(0.009)$ & & & & & $0.035 * * *$ & $(0.009)$ \\
\hline Language performance & $0.783^{* * *}$ & $(0.056)$ & $0.031 * * *$ & $(0.009)$ & & & 0.010 & $(0.009)$ & & & & & 0.008 & (0.009) \\
\hline IQ test & $0.312^{* * *}$ & $(0.058)$ & $0.011^{*}$ & $(0.006)$ & & & 0.007 & $(0.006)$ & & & & & 0.007 & $(0.006)$ \\
\hline Years of schooling & $2.408^{* * *}$ & $(0.136)$ & & & $0.049 * * *$ & $(0.003)$ & $0.038 * * *$ & $(0.003)$ & & & & & $0.037 * * *$ & $(0.003)$ \\
\hline Field of study (Ref. health) & & & & & & & & & & & & & & \\
\hline General & $0.046^{* *}$ & $(0.018)$ & & & $0.171^{* * *}$ & $(0.036)$ & $0.129 * * *$ & $(0.036)$ & & & & & $0.126 * * *$ & $(0.036)$ \\
\hline Engineering & $-0.217 * * *$ & $(0.029)$ & & & $0.140^{* * *}$ & $(0.027)$ & $0.126^{* * *}$ & $(0.027)$ & & & & & $0.124 * * *$ & $(0.027)$ \\
\hline Economics & $0.040^{*}$ & $(0.022)$ & & & $0.134^{* * *}$ & $(0.029)$ & $0.122^{* * *}$ & $(0.029)$ & & & & & $0.120 * * *$ & $(0.029)$ \\
\hline Agriculture and other & $0.041 * * *$ & $(0.012)$ & & & 0.026 & $(0.039)$ & 0.016 & $(0.039)$ & & & & & 0.014 & $(0.039)$ \\
\hline Social science and law & $0.060 * * *$ & $(0.011)$ & & & 0.034 & $(0.038)$ & 0.026 & $(0.038)$ & & & & & 0.025 & $(0.038)$ \\
\hline $\begin{array}{l}\text { Son did not repeat classes in } \\
\text { primary school }\end{array}$ & $0.062 * * *$ & $(0.015)$ & & & $0.063 * *$ & $(0.023)$ & $0.046^{* *}$ & $(0.023)$ & & & & & $0.047^{* *}$ & $(0.023)$ \\
\hline Number of books parents read & $0.739 * * *$ & $(0.071)$ & & & & & & & $0.007^{* *}$ & $(0.004)$ & & & 0.000 & $(0.003)$ \\
\hline $\begin{array}{l}\text { Parents visit concert/museum } \\
\text { regularly }\end{array}$ & $0.303^{* * *}$ & $(0.018)$ & & & & & & & $0.046^{* * *}$ & $(0.015)$ & & & -0.008 & $(0.014)$ \\
\hline SES score neighborhood & $0.626^{* * *}$ & $(0.033)$ & & & & & & & & & $0.066^{* * *}$ & $(0.008)$ & $0.036 * * *$ & $(0.008)$ \\
\hline R-squared & & & 0.105 & & 0.154 & & 0.168 & & 0.007 & & 0.028 & & 0.177 & \\
\hline $\mathrm{N}$ & & & 2238 & & 2238 & & 2238 & & 2238 & & 2238 & & 2238 & \\
\hline
\end{tabular}

Note: $* p<0.1 ; * *<0.05 * * * p<0.01$ Robust standard errors are in parentheses. Earnings of fathers and sons are truncated at the bottom and top 1 percent. All coefficients are

reported as marginal effects. Column [0] shows the results from regressions of each variable on father's earnings, conditional on father's age and age squared.

Columns [1]-[6] show sequential earnings equations; e.g. the effect of cognitive skills, schooling etc. on son's earnings. 
5 How education, cognitive skills, and cultural and social capital account for intergenerational earnings persistence

The second step of the decomposition procedure is reported in Table 5.3. Each variable's coefficient from the earnings regressions of columns [1] to [6] in Table 5.2 is multiplied by the variable's coefficient from the relation with the father's earnings (column [0] in Table 5.2). As Blanden et al. (2007), we summarize these results into different groups: the son's cognitive skills, schooling, and cultural and social capital. The share of intergenerational mobility accounted for by the transmission mechanisms is reported as explained components in the lower part of the table. Correlations between the residuals of the son's earnings equation and the relation with the father's earnings are reported as unexplained components. The specification of cognitive skills in column 1 of Table 5.3 shows that the sum of cognitive skill proxies accounts for about 0.08 points of the 0.24 earnings elasticity coefficient, which is equivalent to 36\%. Schooling (column 2) accounts for about 0.11 points of the 0.24 earnings elasticity coefficient, that is, $45 \%$. Including both specifications in column 3 increases the explained part that accounts for the total intergenerational persistence up to $50 \%$. However, schooling still contributes more to this relation (about 0.08 , or $34 \%$ ) than cognitive skills $(0.04$, or $16 \%)$.

The decline in the importance of cognitive skills from columns 1 to 3 suggests that cognitive skills primarily affect earnings through their influence on schooling. These findings are similar to the results of the study of Blanden et al. (2007) carried out for the United Kingdom. Although the contributions of schooling and cognitive skills are somewhat higher in the Netherlands than in the United Kingdom this suggests certain stability in the extent to which schooling and cognitive skills contribute to intergenerational persistence. ${ }^{26}$ Specifications for cultural capital in column 4 account for only a minor part of the intergenerational earnings persistence. The social capital of the neighborhood, on the other hand, contributes about $17 \%$ (0.04 points, column 5) to intergenerational earnings persistence. Including all predictors in column 6 increases the overall explained part in accounting for intergenerational persistence to $56 \%$, which is only slightly higher than in column 3, where only cognitive skills and schooling are considered. In this combined model, cognitive skills account for $15 \%$ of the intergenerational persistence, social capital for $9 \%$, and schooling takes up the largest part, 33\%, in accounting for the earnings elasticity coefficient $\beta$.

\footnotetext{
${ }^{26}$ In their study, Blanden et al. also use non-cognitive skills as transmission mechanisms. They find that cognitive skills account for 0.09 (29\%) of their 0.32 intergenerational earnings elasticity coefficient. When including cognitive, non-cognitive and schooling variables in one model, these account for $46 \%$ of the intergenerational earnings persistence, 31 percentage points of which is related to schooling and 8 percentage points to cognitive skills (2007: 53f.). We would expect that our results converge to theirs, if we had comparable measures for non-cognitive skills.
} 
5 How education, cognitive skills, and cultural and social capital account for intergenerational earnings persistence

Table 5.3: Accounting for the relationship between father’s earnings and son’s earnings

\begin{tabular}{|c|c|c|c|c|c|c|}
\hline & [1] & {$[2]$} & [3] & [4] & [5] & [6] \\
\hline Math performance & 0.0569 & & 0.0281 & & & 0.0266 \\
\hline Language performance & 0.0243 & & 0.0078 & & & 0.0063 \\
\hline IQ test & 0.0034 & & 0.0022 & & & 0.0022 \\
\hline Sum of cognitive skills & 0.0846 & & 0.0381 & & & 0.0351 \\
\hline Years of schooling & & 0.1180 & 0.0915 & & & 0.0891 \\
\hline \multicolumn{7}{|l|}{ Field of study (Ref. health) } \\
\hline General & & 0.0079 & 0.0059 & & & 0.0058 \\
\hline Engineering & & -0.0303 & -0.0273 & & & -0.0269 \\
\hline Economics & & 0.0054 & 0.0049 & & & 0.0048 \\
\hline Agriculture and other & & 0.0011 & 0.0007 & & & 0.0006 \\
\hline Social science and law & & 0.0020 & 0.0016 & & & 0.0015 \\
\hline Son did not repeat classes in primary school & & 0.0039 & 0.0029 & & & 0.0029 \\
\hline Sum of schooling & & 0.1080 & 0.0802 & & & 0.0778 \\
\hline Number of books parents read & & & & 0.0052 & & 0.0000 \\
\hline Parents visit concert/museum regularly & & & & 0.0139 & & -0.0024 \\
\hline Sum of cultural capital & & & & 0.0191 & & -0.0024 \\
\hline SES score neighborhood & & & & & 0.0413 & 0.0225 \\
\hline Sum of social capital & & & & & 0.0413 & 0.0225 \\
\hline Explained & $\mathbf{0 . 0 8 4 6}(36 \%)$ & $0.1080(45 \%)$ & $0.1183(50 \%)$ & $0.0191(8 \%)$ & $\mathbf{0 . 0 4 1 3}(17 \%)$ & $0.1330(56 \%)$ \\
\hline Unexplained & 0.1536 & 0.1302 & 0.1199 & 0.2191 & 0.1969 & 0.1052 \\
\hline Total & 0.2382 & 0.2382 & 0.2382 & 0.2382 & 0.2382 & 0.2382 \\
\hline
\end{tabular}


5 How education, cognitive skills, and cultural and social capital account for intergenerational earnings persistence

As indicated earlier, we perform an additional test using the log of the house property value of the son in 2008 as a proxy of his permanent income. This is done on a selected sample of sons who are homeowners. To check whether this selection in itself affects intergenerational earnings elasticity and the underlying transmission mechanisms, we repeat the previous analyses for the selected sample. The results are displayed in Tables A5.4 and A5.5 in the appendix: They show virtually the same outcomes as in Tables 5.2 and 5.3. We therefore conclude that the selection on homeownership does not in itself affect the contributions of the different transmission mechanisms.

Table 5.4 presents the results for the analyses using house property value as a proxy for a son's permanent income. The results in columns 1 to 6 show that the relation between the transmission mechanisms and the log of the house property value is generally lower than in the analysis using the son's earnings. The R-squared value in the full model (column 6) is only 0.06, compared to 0.22 in the model with the son's earnings (see Table A5.4). This is not surprising, given the earlier result that the elasticity between a father's earnings and his son's house property value is also much lower. This clearly affects the estimates for the different parameters. For example, an additional year of schooling is associated with only a $1.8 \%$ increase in the log of the house property value, while the effect on son's earnings is $3.8 \%$ (Table A5.4). The coefficient for engineering decreases from 0.133 to 0.013 . The only coefficients that are larger in the analyses with the log of the house property value are repetition of classes during primary school and the SES score of the neighborhood. Not repeating classes increases the house property value by about $10 \%$ and coming from a neighborhood in which the average SES score is one standard deviation higher increases the house property value by about $5 \%$.

Table 5.5 reports the results for the decomposition analysis. The overall proportion that is explained in the full model (column 6) is a little higher than it was in the case for earnings: $62 \%$ instead of 52\% (Table A5.5). More interesting is the shift in the share of the different transmission mechanisms. The share of cognitive skills dropped from $11 \%$ to zero, while the share for social capital increased from $8 \%$ to 31\%. Cultural capital negatively contributes to explaining the relation between a father's earnings and his son's house property value. Social capital plays a stronger role in the transmission from a father's earnings to his son's house property value than in the transmission to the son's earnings. 
5 How education, cognitive skills, and cultural and social capital account for intergenerational earnings persistence

Table 5.4: Relationships between transition mechanisms, father's log earnings and son's log house property value (selected sample)

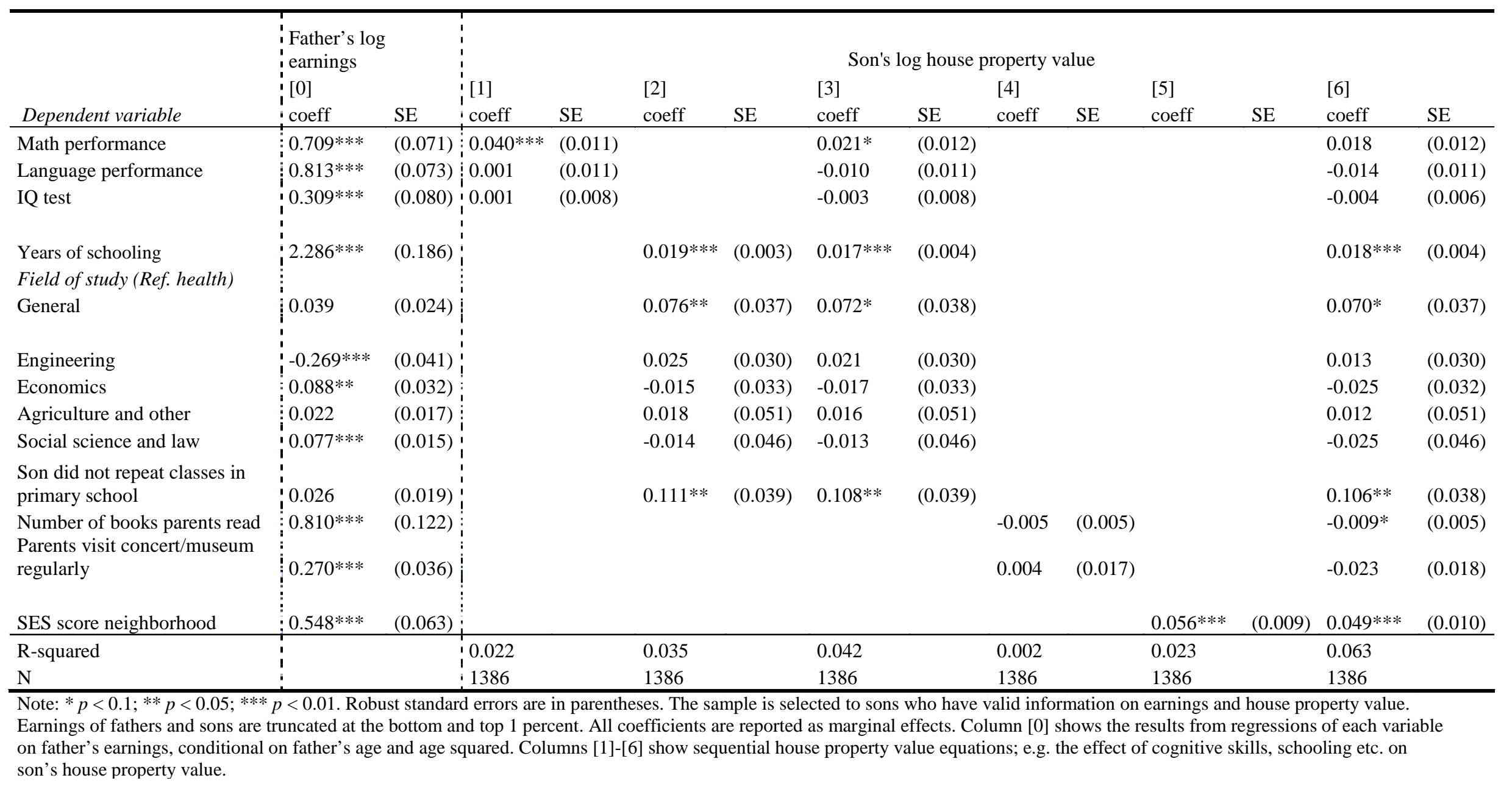


5 How education, cognitive skills, and cultural and social capital account for intergenerational earnings persistence

Table 5.5: Accounting for the relationship between father’s log earnings and son’s log house property value

\begin{tabular}{|c|c|c|c|c|c|c|}
\hline & [1] & {$[2]$} & [3] & [4] & [5] & [6] \\
\hline Math performance & 0.0284 & & 0.0149 & & & 0.0128 \\
\hline Language performance & 0.0008 & & -0.0081 & & & -0.0114 \\
\hline IQ test & 0.0003 & & -0.0009 & & & -0.0012 \\
\hline Sum of cognitive skills & 0.0295 & & 0.0059 & & & 0.0002 \\
\hline Years of schooling & & 0.0434 & 0.0389 & & & 0.0411 \\
\hline \multicolumn{7}{|l|}{ Field of study (Ref. health) } \\
\hline General & & 0.0030 & 0.0028 & & & 0.0027 \\
\hline Engineering & & -0.0067 & -0.0056 & & & -0.0035 \\
\hline Economics & & -0.0013 & -0.0015 & & & -0.0022 \\
\hline Agriculture and other & & 0.0004 & 0.0004 & & & 0.0003 \\
\hline Social science and law & & -0.0011 & -0.0010 & & & -0.0019 \\
\hline Son did not repeat classes in primary school & & 0.0029 & 0.0028 & & & 0.0028 \\
\hline Sum of schooling & & 0.0406 & 0.0368 & & & 0.0393 \\
\hline Number of books parents read & & & & -0.0041 & & -0.0073 \\
\hline Parents visit concert/museum regularly & & & & 0.0011 & & -0.0062 \\
\hline SES score neighborhood & & & & & 0.0307 & 0.0269 \\
\hline Sum of social capital & & & & & 0.0307 & 0.0269 \\
\hline Explained & $\mathbf{0 . 0 2 9 5}(34 \%)$ & $0.0406(47 \%)$ & $\mathbf{0 . 0 4 2 7}(50 \%)$ & $-0.0030(-4 \%)$ & $\mathbf{0 . 0 3 0 7}(36 \%)$ & $0.0529(62 \%)$ \\
\hline Unexplained & 0.0559 & 0.0448 & 0.0427 & 0.0884 & 0.0547 & 0.0325 \\
\hline Total & 0.0854 & 0.0854 & 0.0854 & 0.0854 & 0.0854 & 0.0854 \\
\hline
\end{tabular}

Note: The columns provide the decomposition derived from the equations in Table 4 . We decompose the overall persistence into the contribution of each factor by

multiplying each variable's coefficient in the equation by its relationship with father's earnings (coefficient of column [0] in Table 4) multiplied with the coefficient of column [1]-[6] in Table 4). 
5 How education, cognitive skills, and cultural and social capital account for intergenerational earnings persistence

This indicates that preferences for a certain material status are inherited and can be unrelated to the actual earnings capacity. The choices that people make on the housing market (in terms of what kind of house they buy) are affected by the kind of neighborhood in which they grew up. If they were raised in a neighborhood with a high SES score, it is more likely that they will want to live in similar kind of neighborhood again. This is reflected in the strong effect of the SES score of the neighborhood in which a son grew up on his current house property value.

\subsection{Summary}

In this study we examine intergenerational earnings mobility in the Netherlands and estimate the extent to which transmission mechanisms account for intergenerational earnings persistence. Using a combined set of birth cohort panel data, a large nationwide income survey, and data from tax files, we obtain an intergenerational earnings elasticity estimate of 0.23. Compared to other OECD countries, this estimate is in the lower range of intergenerational earnings elasticity, suggesting that the Netherlands is a country of rather high mobility.

We use the decomposition procedure of Blanden et al. (2007) to assess the contributions of different transmission mechanisms that account for this overall elasticity. The results show that the son's cognitive skills and schooling together account for $50 \%$ of the association between his earnings and his father's. Schooling accounts for the largest part in this association, while cognitive skills mainly work indirectly by affecting the son's highest educational level attained. Including the characteristics of the parents' cultural capital and the social capital of the neighborhood, raises the explained variance of all four transmission mechanisms to $56 \%$ of the association between a father's earnings and his son's. However, the reduced relative importance of each specification in the full model indicates that the transmission mechanisms are correlated, which is especially true for the social capital of the neighborhood and the son's schooling and cognitive skills.

Replacing a son's log of earnings with his log of the house property value yields a much lower elasticity coefficient in association with father's earnings. This is mainly because sons who bought their house in their early 20s profited more from the rise in housing prices than sons who bought their house in their early 30s. Since the latter group is more likely to be the higher-educated sons, with high-earning fathers, this has a depressing effect on the elasticity. The decomposition analysis with the house property value shows that this 
5 How education, cognitive skills, and cultural and social capital account for intergenerational earnings persistence

association is mainly transmitted through the son's schooling and the social capital of the neighborhood, while cognitive skills work entirely indirectly through their effect on schooling. The stronger mediating effect of the social capital of the neighborhood indicates that people prefer to live in the same kind of neighborhood they grew up in, irrespective of the effect on their earnings.

\subsection{Discussion and further research}

The study's main goal was to examine the relative importance of different transmission mechanisms and specifically to explore the relevance of cultural and social capital as transmission mechanisms between a father's and his son's earnings next to the role of cognitive skills and schooling. We found the decomposition model of Blanden et al. (2007) therefore very useful. Within this scope, it is interesting to see that our findings are pretty close to those of the UK study with regard to the relative contribution of schooling and cognitive skills in accounting for intergenerational earnings persistence.

In the introduction we stated that the Netherlands is a knowledge-based society, rather than a class society. That is, the country's prosperity and welfare structure are largely attributed to its social, cultural, and economic openness, allowing for upward mobility to a great extent. However, our findings do not comply fully with this. This study's similarity to the UK study regarding the relative contribution of schooling and cognitive skills suggests that reproduction of intergenerational inequality between high- and low-income families contains a stable element that is independent from the state structure. Education is thereby the main factor that contributes to mobility but also to the reproduction of economic status across generations, since variance in education comes partly from social origin. This is a central finding that is already documented in Blau and Duncan’s work (1967). It implies that policy interventions that aim to improve the socioeconomic outcomes of poorer children may partly be ineffective. Some of the more promising policy actions in stimulating intergenerational mobility target intragenerational improvements, such as enhancing the job opportunities of poorer parents (e.g., by funding self-employment with substantial start-up capital) and the children's intellectual and personal formation in early childhood. Such investments are likely to produce greater revenues in the longer run and can establish assets that persist over generations. Further research could be directed toward the impact of intervening in the investment strategies of schools, parents, and children in the upward mobility of children, especially for those from a poorer social background. 
5 How education, cognitive skills, and cultural and social capital account for intergenerational earnings persistence

Our findings show that $56 \%$ of the earnings elasticity can be explained by the transmission mechanisms in the model, while $44 \%$ remains unexplained. This suggests that other (unobserved) mechanisms may play a role, such as the transmission of specific personality traits and attitudes or access to professional networks that are related with income transfer or, more specifically, wealth transfer. Studies on family wealth suggest that financial and intellectual family assets "guarantee" stability in investments in the educational and occupational careers of the offspring, among others, by the intergenerational transmission of personality traits (e.g., Lindh and Ohlsson, 1996; Blanchflower and Oswald, 1998). In particular, personality traits such as risk tolerance and entrepreneurial skills-as well as the spillover effects of family wealth that are related to a father's earnings-are likely candidates to account for part of the intergenerational persistence that remains unexplained (e.g., Rauch, 1993; Banabou, 1996). Future research should focus more on these other types of transmission mechanisms.

Another issue to be addressed in future research is the problem of measurement error. Although the decomposition model we use is attractive in that it is very straightforward, a drawback is that the results depend on, among other things, the extent to which concepts are well measured. Put simply, if education is measured with more accuracy than cognitive skills, education will turn out to explain more of the intergenerational earnings persistence. Other models, such as LISREL, could separate the structural from the measurement model, thus allowing concepts to be measured with different levels of reliability. 
6 Conclusion 


\subsection{Summary}

The aim of this thesis was to explore theoretically and empirically the role of social and cognitive factors that are related with the educational attainment process and subsequent labor market outcomes of young people in the Netherlands. This thesis took a vertical perspective on social class differences in educational outcomes and related changes over time, a horizontal perspective on gender differences in labor market returns, and an intergenerational perspective on transmission mechanisms accounting for the earnings persistence between fathers and sons. Furthermore, we combined sociological theories with economic approaches with the interest to open up an additional view on the social stratification debate. A strong point regarding the empirical analyses was the use of large and unique data sets that enabled us to compare panel information for different birth cohorts on the schooling level and to combine them with administrative data for analyses on labor market level. The central research questions addressed were:

- What is the relative role of direct and indirect effects of social background in explaining educational choices and attainment, and thus the size of IEO?

- What can be said about the underlying mechanisms associated with educational attainment and their relationship with labor market outcomes?

- What is the relative importance of transmission mechanisms that account for the association between a father's and his son's earnings?

In the following, I give a summary on each chapter and a short review about the contribution to the existing literature.

\subsubsection{How social background affects educational attainment over time in the Netherlands}

The social stratification literature states that social background is a strong factor explaining social inequalities in educational attainment (e.g. Blossfeld and Shavit, 1993). A factor less considered is the role of cognitive skills, mediating the correlation between social background and educational attainment. Boudon's approach on primary and secondary effects of social stratification (Boudon, 1974) targets this issue and is described and analyzed empirically in Chapter 2. Primary effects relate to students' cognitive skills differences, dependent on their social background. Secondary effects relate to educational choices 
students make depending on their social background and (previous) cognitive performance. We used a decomposition model that is based on a concept of Erikson et al. (2005) and Jackson et al. (2007) to investigate the relative importance of primary and secondary effects at two major school transitions over four Dutch school cohorts. The first transition concerned that from primary to higher secondary education at age 12, while the second one considered the conditional transition from higher secondary to higher postsecondary education at age 17 or 18. At the first transition we examined the probability of entering either the pre-university track (VWO) or the precollege track (HAVO), and the probability of entering VWO only. This distinction was made, as we assume social background differences in choosing between the more (VWO) and the less (HAVO) prestigious tracks in higher secondary education. Similarly, we distinguished at the postsecondary education level between the probability of entering either the university track (WO) or the less prestigious vocational college track (HBO), and the probability of entering WO only. In addition, we looked at unconditional transition probabilities of all students making the transition to either $\mathrm{HBO}$ or $\mathrm{WO}$, and WO only. We found that cognitive performance is correlated with social background and that students with higher educated parents are more likely to choose straight career paths from VWO to WO over time, which characterizes these tracks as an elite path. While transitions and completion rates for (pre)university education remain fairly constant over time, vocational colleges gain in popularity, especially among less privileged children. This is mostly visible by increasing transition rates from $\mathrm{HAVO}$ to $\mathrm{HBO}$, but also - to a lower extent - from within-level transitions at the postsecondary level, from upper secondary vocational education $(\mathrm{MBO})$ to $\mathrm{HBO}$. We found that secondary effects' relative importance at the transition to higher secondary education is on average $45 \%$ and decreases across cohorts, which is mainly responsible for the slight overall decrease of secondary effects over time. However, at the transition to postsecondary education the size of secondary effects increase again, being, on average, 53\% in the unconditional model and $81 \%$ in the conditional model. These large proportions suggest stemming from the selection process at the first transition and the fact that disadvantaged students rarely take the opportunity to repair 'erroneous' track decisions at later transitions. Although Dutch policy interventions towards performancerelated selection were quite successful at the first transition, our findings showed that this is offset at later transitions. IEO is still to a considerable extent related to social background differences in the choices students make, conditional on performance.

Our findings about the relative importance of secondary effects are quite consistent with a similar study, carried out by Kloosterman et al. (2009) for the Netherlands. However, 
we additionally stressed the fact that straight career paths from pre-university to university education still remain fairly exclusive for children with higher educated parents. Furthermore, our findings contribute to a cross-national comparison of eight countries, each examining the relative importance of primary and secondary effects. ${ }^{1}$ The cross-country analysis, carried out by Jackson and Jonsson (2013), thereby gives new insight in the variation in IEO. The study shows that the Netherlands and Germany are similar in generating relatively high levels of IEO at the first transition. This seems due to the fact that the education systems of both countries are highly stratified and highly selective (Jackson and Jonsson 2013:320). At the second transition, however, the Netherlands, England, and France, compared to the other countries, produce the lowest levels of IEO for those who are at risk of making the transition. Primary effects thereby vary much less in magnitude across countries and are less affected by the structure of a specific educational system than secondary effects. The authors suggest that in each country a floor level of IEO exists, coming from social background differences in abilities and aptitudes and are reflected in scholastic performance. Above that level, secondary effects generate the variation in the overall level of IEO per country and thus are largely responsible for the variation in the size of IEO across countries (cf. Jackson and Jonsson 2013:327). If secondary effects increase in magnitude, the level of IEO also increases. The size of secondary effects thereby is driven by the country-specific institutional features of the education system. Moreover, the authors assume that structural features of the education system are linked with the inequality level of (economic) conditions in a country and this interplay generates the size of primary and secondary effects and the level of IEO (Jackson and Jonsson, 2013).

\subsubsection{Social background's effect on educational attainment: Does method matter?}

Chapter 3 examined the robustness in the size of social background's primary and secondary effects by applying three different statistical models. The models under use were a counterfactual model, a linear regression model, and a structural equation model (SEM). Each model has different assumptions and implications regarding the relative weight of IEO.

\footnotetext{
${ }^{1}$ Our study for the Netherlands is part of an international book project (Jackson, 2013) realized by members of the Network of Excellence of Economic change, Quality of life and Social cohesion (EQUALSOC), funded by the $6^{\text {th }}$ Framework Program of the EU. The countries under study are: Germany, the Netherlands, France, Italy, Sweden, Denmark, England, and the United States. Each country describes its educational system and analyzes, under the consideration of the specific institutional aspects, the relative importance of primary and secondary effects on the basis of the same empirical method.
} 


\section{Conclusion}

We applied these models on analyzing changes in primary and secondary effects over three school cohorts and at the entrance and completion stages of secondary and postsecondary education, as well as on the social background's total effect on the highest degree attained, unconditional on previous educational transitions.

We found that secondary effects have a decreasing impact at the first transition over time, but a rather stable and in some cases increasing impact at the educational career's later stages. As a result, the cumulative share of secondary effects on educational attainment is stable over time, at least when examining the last two cohorts.

Furthermore, we found that secondary effects' relative size is larger when completing a track than when entering a track. Using linear regression and counterfactual models thereby yield comparable results for the size of primary and secondary effects. Using SEM, which corrects for measurement error in the performance and social background variables, however, suggests that secondary effects are overestimated in the two other models: The relative importance of choice-related differences is considerably lower at the transition to secondary education and at the general highest educational level attained. Secondary effects amount to some $55 \%$ of social background's total effect, when ordinary least squares or counterfactual models are used, and to some $45 \%$ when structural equation modeling is used. This suggests method does matter for numerical closeness. Nevertheless, all three models confirm an increase in the relative size of secondary effects at postsecondary education and thus confirm Boudon's claim that secondary effects are reproduced by an equal share at each transition, which results in a cumulative increase until the final educational stage. As both primary and secondary effects contribute to IEO, policy makers should focus, more than before, on reducing performance-related differences at the transition to secondary education and choicerelated differences at the transition to and throughout the postsecondary education level.

This chapter's contribution lied, first, in testing for the results' robustness by applying different estimation techniques. We show that secondary effects are overestimated if we do not correct for measurement error, which in turn can lead to the wrong policy implications. Second, unlike most other studies which have analyzed social background's primary and secondary effects, we examined both transition and completion stages throughout the educational career. Our results show that secondary effects' relative importance indeed alters at these different stages, giving insight in the rather surprising result that a successful completion in higher education is more determined by choice-related than by performancerelated processes. Third, by testing hypotheses on the basis of different social inequality theories, we notice differences to Blossfeld's and Shavit's cross-national study (1993). The 
authors compare changes in IEO over 13 countries, denoting a declining trend in the association between social background and educational attainment for the Netherlands. By taking additionally cognitive performance in our analysis into account, we found little change in IEO over the last two cohorts in the Netherlands, while especially higher postsecondary educational levels remained fairly exclusive for advantaged students. Our results are therefore mostly in line with arguments of rational action theory (e.g. Breen and Goldthorpe, 1974) and reproduction theories (e.g. Bourdieu, 1973; Collins, 1971) and refute modernization theory, as we do not observe a considerable decline in the association between social background, cognitive performance, and educational attainment over time.

\subsubsection{Education, cognitive skills and earnings of males and females}

Chapter 4 focused on gender and earnings returns and addressed the question whether males and females are rewarded to the same extent for their cognitive skills at the labor market, even if they are already sorted into "appropriate” educational levels and study fields. We therefore investigated the relationship between three proxies of cognitive skills, measured at age 12, and earnings of males and females, measured at age 35, conditional on their educational levels attained. The proxies for cognitive skills comprise two standardized school achievement tests in math and language, and a non-verbal intelligence test. The data set used combines longitudinal information of a Dutch school cohort survey with administrative data from tax files and municipalities. The data also provide individual information about social background and schooling during childhood, as well as about the job sector, partner characteristics and the family composition at age 35, for which we additionally controlled in separate earnings regressions for males and females.

We found cognitive skills and specifically math skills are rewarded at the labor market, but more for females than for males, in general and conditional on education. The main factor driving this result is that cognitive skills appear to predict schooling outcomes better for males than for females. When specifying the earnings regressions by educational level and controlling, among others, for study field, the cognitive skills coefficients for medium and higher educated males are not significant anymore. In contrast, for lower educated males and for females of all educational levels we found math skills still being highly significant in predicting earnings. Moreover, for females we found a residual impact of math skills on earnings differing per study field. This residual impact is significant for lower and medium educated females choosing agricultural studies compared to general 
studies, and for higher educated females choosing economic studies compared to other studies (i.e. social studies, humanities and arts). We concluded that for males' earnings skills are largely a function of schooling. Skills play a role but only indirectly, as they are fully converted into males' years of schooling and specific study fields. Study fields, such as engineering, that correlate highly with math and IQ scores, are more often chosen by males than by females, conditional on education. Differences in years of schooling between males and females are rather minor, but females' selection in education is less based on their cognitive skills. Their educational success appears, stronger than for males, to result from other factors, such as motivation or diligence. However, although females' cognitive skills may not be fully recognized in the Dutch education system, they are recognized at the labor market.

Analyzing males' and females' earnings returns to cognitive skills, conditional on education is, to our knowledge, still rare in the social inequality literature. This chapter aimed to shed more light on potential gender differences in labor market outcomes that are driven by cognitive skills directly, and indirectly, by the educational selection process based on cognitive skills. The fact that we used three different cognitive skills proxies, measured at a relatively early age, gave us the opportunity to analyze to what extent they differ in magnitude and significance in predicting educational attainment and earnings. Furthermore, we included a set of contextual factors in the earnings regressions, such as parental background, and partner and family characteristics later in life, to reduce omitted variables bias, regarding the returns to cognitive skills estimates (Zax and Rees, 2002).

\subsubsection{How education, cognitive skills, cultural and social capital account for intergenerational earnings persistence}

Chapter 5 estimated the intergenerational earnings mobility in the Netherlands and examined to what extent transmission mechanisms, such as sons' education and cognitive skills, the family's cultural capital and the social capital in the neighborhood account for the intergenerational earnings persistence between fathers and sons. We applied a decomposition model of Blanden et al. (2007) and found that cognitive skills and education account for 50\% of the father-son earnings association. We run an additional test, in which we replaced son's earnings by son's house property value as a proxy of his permanent income. The test gave virtually the same results as when son's earnings were used: Education accounts for the largest part, while cognitive skills mainly work indirectly through educational attainment. 
Social capital of the neighborhood and cultural capital of the parents account for an additional $6 \%$ of the intergenerational earnings persistence. What mechanisms do account for the remaining $44 \%$ we could not observe, but it is conceivable that the transfer of specific personality traits and spillover effects of family assets play a role in accounting for the association between a father's and his son's earnings.

Moreover, our findings regarding education and cognitive skills are quite similar to those of the UK study of Blanden at al. (2007). Although the Netherlands is characterized as an open society allowing more equality in social opportunities than in the UK, the similarities in the two countries suggest that part of the intergenerational earnings persistence accounted for is unaffected from a specific society's structure. This supports a central finding in the stratification literature, namely that education contributes to a major part to reproduction of economic status (cf. Blau and Duncan, 1967). In a next step, the empirical realization of the concept has nevertheless to be put to the test, as correlations between the mechanisms bias the outcome. The application of alternative estimation techniques to reduce measurement error therefore is a target for future research.

By using the same methodology and comparable data information as in Blanden et al. (2007), our study allowed discussing the findings in comparison to those of the UK study. As a result, we stated that education and cognitive skills function as transmission mechanisms which are susceptible to account with relative similar sizes to intergenerational earnings persistence across countries. Our newly introduced transmission mechanisms to the empirical approach, parents' highbrow activities as a proxy for the son's cultural capital and the social status of the neighborhood as a proxy for the son's social capital, combine economic and sociological aspects of intergenerational mobility research. In our study these mechanisms did not account strongly for the association between a father's and his son's earnings, but they motivate to look for other variables reducing the unexplained part that accounts for the intergenerational earnings persistence in our research.

\subsection{Discussion}

\subsubsection{Social inequality and the role of education}

The application of Boudon's approach of primary and secondary effects in this thesis was helpful, in order to get a better understanding about the processes underlying the relationship between social background and educational attainment. This approach not only targets the 


\section{Conclusion}

cultural and economic differences between social backgrounds, but also attaches an important role to the education system. More precisely, it links to a central issue in the sociological literature; the legitimacy of a national education system and in how far it fulfils the function of providing equal opportunities for social mobility.

As Boudon (1974) introduced and Jackson (2013) outlines, the understanding this thesis has from social inequalities in educational attainment is based on the consequences of social background differences both in academic performance and in the educational choices students make, conditional on performance. The level of IEO is therefore intrinsically tied to the structure of the education system. The Dutch education system is characterized by a highly stratified and highly selective system, where both features are connected to secondary effects to a different extent (Jackson and Jonsson, 2013). An early tracking and highly stratified system offers variation in education among students and can produce useful variation at the labor market (Gamoran, Nystrand, Berends, and LePore, 1995; Korthals, 2012). However, such a system is also likely to produce larger proportions of secondary effects, as (early) track decisions for more prestigious tracks are social background driven and, as this thesis shows, are consequential for the track decisions in the later educational career. $^{2}$

If a stratified education system is also characterized as highly selective, it can raise a number of further issues. One issue relates to the standards that are being used to determine track placement. In the Dutch education system track placement is in principle based on standardized performance tests, which, by definition, is a merit-based concept that should lower secondary effects. Korthals (2013:21) indeed found that students in highly differentiated systems perform best when schools always take into account prior performance to decide on students' educational placement. Weaknesses in the system, however, occur when the placement of students is based on other criteria or when criteria are lacking. This is the case, for example, when there is too much leeway for negotiation in track decisionmaking. This opportunity is often used by better-off families placing their children in the highest educational levels possible, even if performance is not adequate. Jackson and Jonsson (2013) state that such compromises in a selective system promote secondary effects, also because children from disadvantaged backgrounds do not behave in the same way and choose, as this thesis shows, rather lower educational levels, or even drop out (Traag, 2012).

\footnotetext{
${ }^{2}$ It suggests that - albeit politically supported - summarizing HAVO and VWO as coequal tracks under higher secondary education, and $\mathrm{HBO}$ and $\mathrm{WO}$ as coequal tracks under higher postsecondary education masks the social selection into these tracks and the actual consequences it has on differences in the economic outcome later in life.
} 


\section{Conclusion}

The results of the thesis show that the relative importance of secondary effects increases again at postsecondary education level implying that advantaged groups pursue their educational preferences and use the opportunities provided by the education system adequately.

Another issue making a selective system vulnerable is related to the number of selection moments and whether or not repairs are possible. Turner (1960) describes two ideal types that are helpful in understanding differences between educational systems: the sponsored mobility system and the contest mobility system. In the sponsored mobility system selection is a one-shot event, and once students are placed in the higher track, their educational attainment is 'guaranteed' (hence 'sponsored'). But students who fail to pass this hurdle are not allowed to re-enter in the higher tracks. In the contest model, however, selection is continuous and 'repairs' are always possible. It is intuitively clear that the sponsored mobility system produces more waste of talent (Van der Velden, 2011): some students will get promoted to the higher track who do not deserve this (the 'false positives'), while others are deterred to the lower tracks who actually have the abilities to follow the higher track (the 'false negatives'). As Van der Velden (2011) indicates, having multiple rounds of selection improves the reliability strongly (thus reducing the number of 'false positives') and if the system allows to re-enter higher tracks at a later moment in the career this reduces the number of 'false negatives'. The findings for the Netherlands in this thesis suggest that at least repairs of 'erroneous' track decisions are hardly deployed later in the career.

An education system's legitimacy also has to be put to the test with regard to correlations between social background and academic performance. A “socially blind” meritbased selection in educational tracks is to question, if academic ability is positively related with family background. Policies aiming to reduce inequality by assessments based on performance tests will be - at least to some extent - in conflict with the fact that primary effects do contribute to IEO. Moreover, findings of this thesis suggest an increase in primary effects at the first transition over time, meaning that students from higher educated background extent their performance-related advantages to less privileged groups. The education system, intentionally or not, supports this and thereby seems to be related to the (changing) level of inequality of economic conditions. As Jackson and Jonsson (2013) explain, "greater economic inequality (...) both increases the capacity of advantaged classes to provide high-quality child care and extra tuition, increasing the size of primary effects, and changes the decision calculus surrounding educational transitions, increasing the size of 
secondary effects” (ibid:313). As regards the findings in Chapter 2 of this thesis, secondary effects' relative proportions accounting for overall IEO are slightly decreasing over the observation period, but they turn out to be only marginally lower than those of primary effects. Such a ratio is a challenge for the education system's legitimacy, especially if choicerelated differences are supposed to be easier tackled by state interventions than performancerelated differences.

\subsubsection{Gender and the role of education}

An intriguing finding of this thesis was that males' cognitive performance measured at age 12 largely converts into “appropriate” educational levels, while females' talents appear not to be fully recognized in the education system. We find that males and females do not significantly differ in their years of schooling, which implies that females compensate with features other than cognitive skills to achieve the same educational levels. It is conceivable that these features are related to non-cognitive skills being beneficial in the education system, such as motivation, diligence, or perseverance. It poses the question, however, if the education system applies other standards to males than to females and whether these standards may be jointly responsible for (absolute) earnings differences between males and females. We find earnings returns to cognitive skills, and particularly to math skills, are stronger for females than for males, conditional on education and controlled for study field, job sector, and family characteristics. As the residual impact of math skills on earnings per study field is also significant, one explanation can be that females, on average, are selected in study fields that do not fully match with their talents. This 'reserve' of talent is nevertheless rewarded on the labor market by employers. They reward them for their 'additional skills' exceeding the required qualification on the job. We could not test for occupation, ${ }^{3}$ but scholars in sociology argue that the only differences observable between males and females are associated with differences in occupational destinations. ${ }^{4}$ It raises once more the question whether females' talents are not enough promoted by the education system, for example, by not directing them to more lucrative study fields. Or, in contrast, are these results linked to gender specific preferences in the educational career that lead to a 'reserve' of talent for females?

\footnotetext{
${ }^{3}$ Instead, the data provide job sector variables, but they give no indication on job positions within the sector. Including the sector information as controls in the analysis did not change the substantive results.

${ }^{4}$ Hout and DiPrete (2004) summarize the findings collected over years of researchers who presented their work at the Research Committee on Social Stratification (RC28), of the International Sociological Association.
} 


\subsubsection{Cognitive skills measurement and individual outcome}

The data used in this thesis provide three proxies for cognitive skills, which allowed to show that achievement tests in math and language predict earnings better than a non-verbal intelligence test. It supports findings of Almlund et al. (2011) and Borghans et al. (2011), who state that achievement tests take up a substantial part of personality indicators that eventually play an important role in predicting life outcomes. These indicators are most likely connected with non-cognitive skills, such as motivation and perseverance, being beneficial at the labor market over and above cognitive skills (Heckman et al., 2006).

However, one issue related to these achievement tests concerns the purpose and context in which the tests are measured. Allen and van der Velden (2012:32) argue that the assessment of school performance runs the risk to be linked too tightly to interests of educational policy and practice. This may result in a misalignment between teaching and learning, generating a process of "teaching to the test", 5 and a contest atmosphere in which all actors involved are interested to win the contest rather than focusing on talent with adequate learning concepts and contents.

A second issue is that these tests may be vulnerable to a correct measurement if there is a one-time assessment at a relatively early age only (see section 6.2.1). As Van der Velden (2011) argues, repeated tests (e.g. at later stages of the educational career) and a permeable system with good return possibilities would reduce measurement error and the proportion of students who are misplaced in the higher educational levels.

A third issue relates to the uni-dimensionality of the selection. The education system is typically geared towards students that have similar talents for all subjects. Students have to be good in both language and math and science in order to proceed to the highest tracks. But, although there are clear correlations between the test scores in the different subjects, these are not perfect. This is illustrated by the results in Chapter 4 of this thesis, showing significant gender differences in language and math performance. This can be even more extreme for students who have highly specialized talents in one subject, while clearly failing in others. It raises the question if these "exceptionally talented students" (Allen and van der Velden, 2012:44) are well assessed and well placed in a system that is arranged as a more generalized concept that fits the broad average of students. Those talents may not be sufficiently stimulated and developed in a system as present. Alternative ways of selection programs

\footnotetext{
${ }^{5}$ It is likely that such a procedure also maps more obviously social differences than pointing to putative intellectual qualities, as better-off students are more used to certain learning habits (cf. Bourdieu, 1973) advantaging them in the tests.
} 
would be thinkable, which are more tailor-made by school subjects and not by age or track, giving the opportunity to excel in the talented field and the time to catch up in others.

\subsection{Policy implications}

Vertical differences in the Netherlands - regarding educational attainment and the intergenerational reproduction of economic status - remain relative and absolute observable in this thesis. As education is a central factor contributing to these differences, policy actions should aim to reduce the level of IEO by targeting both performance-related differences and choice-related differences of social background.

As yet, the introduction of CITO tests - aimed to assess primary school students' placement in secondary education tracks on the basis of achievement tests - were already successful in reducing secondary effects in the Netherlands. However, programs that also reduce social differences in performance should be promoted and exerted already at preschool age to be effective. Studies on returns to such programs show that they are successful in improving both cognitive skills and personality skills (e.g. Heckman, 2006; Heckman et al., 2012). Although some scholars show concern that such actions demand high costs and gear into private areas that should not be subject to state interventions (e.g. Jackson et al., 2007; Swift, 2005), early interventions hold good prospects of later educational and occupational success that pays off the investments (e.g. Cunha and Heckman, 2010). As enhanced cognitive and personality skills are related to increasing achievement in performance tests and schooling, such interventions also suggest to exert implicit influence on secondary effects, by boosting motivation and aspirations (Heckman et al., 2012). ${ }^{6}$ Choices for higher educational levels, however, are also subject to economic constraints and related with higher costs and risks for students from disadvantaged backgrounds. The increasing secondary effects at the transition and completion stages of post-secondary education, presented in this thesis, point this out. Policies could intervene with financial incentives for talented students from lower social backgrounds, such as reduced tuition fees when enrolling in universities and/or by professional guidance and counseling throughout their educational career. The latter pursues the goal to increase these students' transitions to more prestigious tracks by providing them with comprehensive information about their

\footnotetext{
${ }^{6}$ Our estimations on counterfactual transitions rates to higher education show that students from disadvantaged backgrounds clearly increase their transition propensity if they (would) have the same average performance as students from advantaged backgrounds.
} 
abilities and chances of success at higher educational levels (Jackson and Jonsson, 2013). Such actions can be usefully supplemented by repeated performance tests at later stages, reducing track selection error, and good return possibilities in the tracking system, which is underdeveloped in the Dutch education system (Van der Velden, 2011). These tools could help to readjust both professional guidance and individual decisions throughout the educational career and, in sum, are likely to make students' educational decisions less dependent on family background.

A similar strategy can be applied to males and females, regarding a more tailor-made, professional guidance on their educational planning. Especially talented girls should be boosted early in their school career to use their full potential, for example, by supporting them in their decision for study fields that meet their skills optimally. Integrating girls stronger in boys' dominated courses, like engineering, however, may fail due to gender and age specific peer-group orientations. One possible strategy would be to make the environment more attractive to girls and to increase the female share by starting active recruitment with subject specific topics that can be related to issues girls are interested in, but lying just outside the core subject area. ${ }^{7}$ Another strategy is to start professional guidance not only as early as possible in the educational career, but also in a context offering subject oriented differentiation rather than track oriented differentiation. This would characterize an extremely individualized system, possibly claiming more resources for qualified staff than the system as yet.

Systematic, constant and possibly increasing (financial) investments in education seem to be necessary to respond to increasing educational participation and the need for specifically qualified individuals. An education system that provides a tailored program for all groups of students would offer sensible solutions to the new demands and challenges at the labor market.

\subsection{Strengths and weaknesses of the data}

The empirical strength of this thesis lies in the use of large and unique data sets, coming from Statistics Netherlands. The four school cohort surveys used here are large-scale representative panel studies that provide comparable data information over time. Information on schooling,

\footnotetext{
${ }^{7}$ For example, one could think of topics comprising the technical setup for creating an internet blog, the functioning of camera and photo technology, or analyzing the physical and chemical processes of dying hair or manipulating food.
} 


\section{Conclusion}

skills, and social background is provided for nearly 20.000 students per cohort and collected from the students, their families, the schools and the teachers. In particular, enrolment and examination data are derived from school administrations. That is, general attrition in the surveys is not a big issue when following individual educational careers. Moreover, these data can be matched with administrative data, coming from tax offices and municipalities, and other national surveys which allow extending empirical analyses and raising new and further research questions.

Nevertheless, the data also revealed some drawbacks for the analyses in this thesis, of which four have been of greater relevance. One first issue concerns the measurement of cognitive performance at different points in the educational career. Although the school cohorts of VOCL 1989 and VOCL 1993 provide extra cognitive skills tests at age 15, next to the CITO tests at age 12, these tests are not suited to compare students' performances over the educational career and across different educational tracks at later transitions. ${ }^{8}$ Data information about comparable cognitive skills tests at later transitions or school grades as alternative proxies for scholastic performance may provide support for the findings presented here, such as whether returns to cognitive performances are robust over the educational career and/or to different skills measures.

Second, the survey design of school cohort SLVO 1982 deviates from the other three school cohorts that have been under study. Not only the sample size is smaller, but also, for example, information on parental education was collected in a different way than in the other three cohorts. The latter resulted in comparison issues across the cohorts and the decision to leave out this sample when robustness tests are carried out regarding the magnitude and relative contribution of primary and secondary effects.

Third, the cohort surveys and administrative data provide little or no occupational information of parents and their children. However, standardized social class schemes that are based on occupational information, such as the EGP class scheme (Erikson, Goldthorpe, and Portocarero, 1979), would serve as an alternative and internationally comparable measure for social origin, next to parental education. Moreover, occupational status gives over and above earnings information a better understanding about labor market returns to cognitive skills and educational attainment.

Fourth, the data do not give much leeway to test for causal effects between cognitive skills, schooling, and labor market outcomes. Although the cohort design provides a

\footnotetext{
${ }^{8}$ For more explanation about a problematic comparison of the cognitive skills tests at age 12 and age 15 , see Footnote 17 in Chapter 2.
} 
chronological order in events, which enables us to estimate earlier cognitive performance in predicting subsequent educational and labor market outcomes, this does not ensure causal relations. The use of experiments or an instrumental variable approach would be helpful tools examining causality, but the survey design does not provide such settings.

\subsection{Future research}

The inclusion of cognitive skills, cultural and social capital variables in this thesis provided material to detect some of the puzzle of how education contributes both to mobility and reproduction in social destinations. However, the findings remain on a rather descriptive level, while a substantial part of unexplained variance is still left due to unobserved factors. To overcome data and measurement limitations in future research, it will be helpful to apply data that allow testing for causal relations between family background, cognitive skills, schooling, and labor market outcomes (see section 6.4). Another step for future research would be to combine the data used here with subsamples that enclose more and alternative information on cognitive skills for children at pre-school ages and their parents. The data at pre-school ages should provide comparable information by standardized skills tests, ${ }^{9}$ to function either as robustness check for the tests in the school cohort surveys and with regard to reducing measurement error in primary effects, or optionally document skills development stemming from changing environments over the life course. Having cognitive skills information of their parents, in addition, would help to differentiate cognitive skills acquisition by genetic inheritance or socialization processes, ${ }^{10}$ which would improve our understanding of the mediating function of cognitive skills between social background and education.

Furthermore, it would be interesting to exploit more and comparable information across cohorts on students' non-cognitive skills and later earnings and occupational destinations. Information on students' personality traits, attitudes and aspirations could provide better explanations for (social) differences in educational transitions. Matching the respondents' non-cognitive skills with occupation and earnings information could improve our understanding of gender differences in labor market returns, while using this information

\footnotetext{
${ }^{9}$ The measurement of cognitive skills and ability is a general challenge in the economic and sociological literature. Scholars so far do not agree to one method to measure IQ and tend to differentiate between several kinds of ability, for which they develop sub-tests which measure a small part of ability at best.

${ }^{10}$ Plug (2004), for example, tested for the effects of unobserved inherited abilities on child's schooling. He found that the association between mother's schooling (not father's) and child's schooling disappears, when it is controlled for inherited abilities and assortative mating.
} 


\section{Conclusion}

across cohorts enhances our research on whether contributions of transmission mechanisms in accounting for intergenerational earnings persistence are changing or not.

An implicit but central concern of this thesis was to give some answers to the societal function of the education system and how well it lives up to expectations of lowering IEO in the Netherlands. Including a comparative analysis on country differences in educational attainment, in addition, assesses the national findings in a broader context, allows generalizations, and helps to revaluate the strengths and weaknesses of the national education system. In part, we could take this into account already, as the findings of one study in this thesis contribute to an international project aiming to compare the level of IEO across countries and time (see Chapter 2, and Jackson, 2013). Nevertheless, this perspective can be extended in future research to country comparisons of education systems, in context of different labor market returns to education and cognitive skills. Data that can be used for appropriate comparisons between countries are provided by the PIAAC project or the REFLEX project. ${ }^{11}$ Furthermore, it will be sensible to combine this with more in-depth information from a two-country comparison, for example, between Sweden and the Netherlands. These countries' education systems are similar in structure but clearly differ in the age at entry to secondary education. Moreover, there are Swedish school cohort data surveyed in a similar panel design and at a similar time as school cohort data for the Netherlands, while both surveys can potentially be matched with labor market information. Extending this country comparative analysis to labor market outcomes may change the perspective that was prevalent on an education system's legitimacy, namely from providing "equal opportunity" to whether the "rate of return" to education is high enough to justify its cost (cf. Hout and DiPrete, 2004:14). Comparative analyses across countries and preferably over time are therefore aimed in a next step to empirically confirm or contradict general developments in this pattern.

\footnotetext{
${ }^{11}$ For more information, see: www.oecd.org/els/employment/piaac and www.reflexproject.org.
} 
Appendix 


\section{Appendix}

Figure A2.1: Transition and completion rates in secondary and postsecondary education, 1977 school cohort

Age

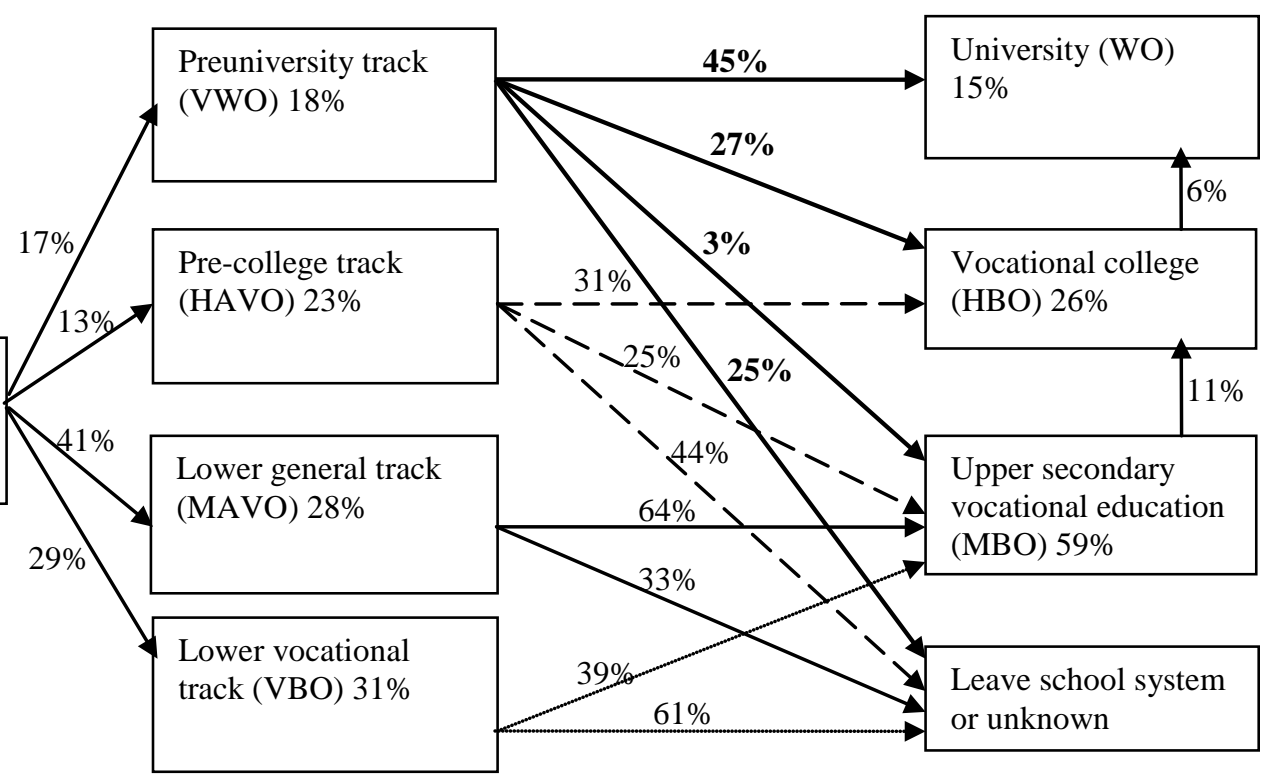

Source: CBS cohort 1977; our calculations.

Note: The arrows represent the transition from one track to another track and add up to $100 \%$. The figures in the boxes represent the highest completed level of secondary or postsecondary education. These can differ from the inflow as a result of upward or downward mobility. For the transition at age 12 we have information for all students. For the transition at age 17-18 we lack information for students who dropped out of the panel or who left school altogether; they are taken together in the category "Leave school system or unknown.” 


\section{Appendix}

Figure A2.2: Transition and completion rates in secondary and postsecondary education, 1982 school cohort

Age

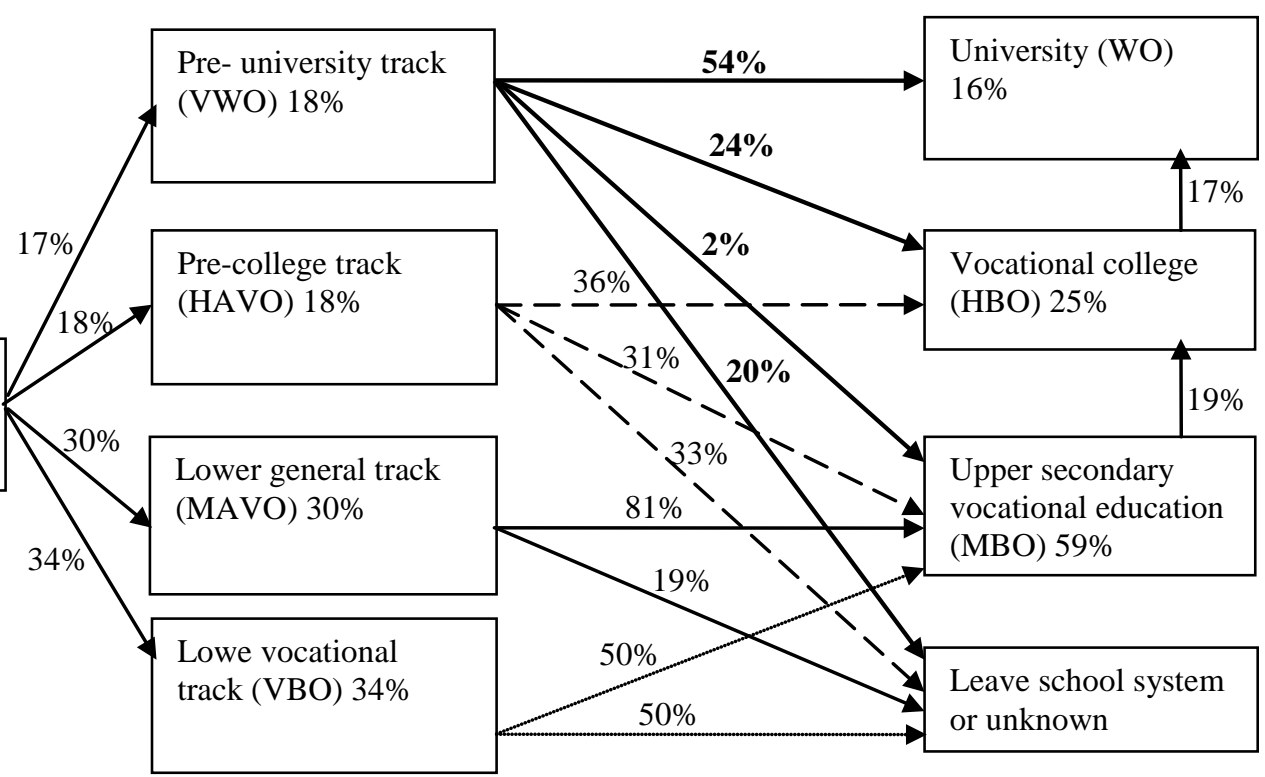

Source: CBS cohort 1982; own calculations.

Note: The arrows represent the transition from one track to another track and add up to $100 \%$. The figures in the boxes represent the highest completed level of secondary or postsecondary education. These can differ from the inflow as a result of up- or downward mobility. For the transition at age 12 we have information for all students. For the transition at age 17-18 we lack information for students who dropped out of the panel or who left school altogether; they are taken together in the category "Leave school system or unknown." 


\section{Appendix}

Figure A2.3: Transition and completion rates in secondary and postsecondary education, 1989 school cohort

Age

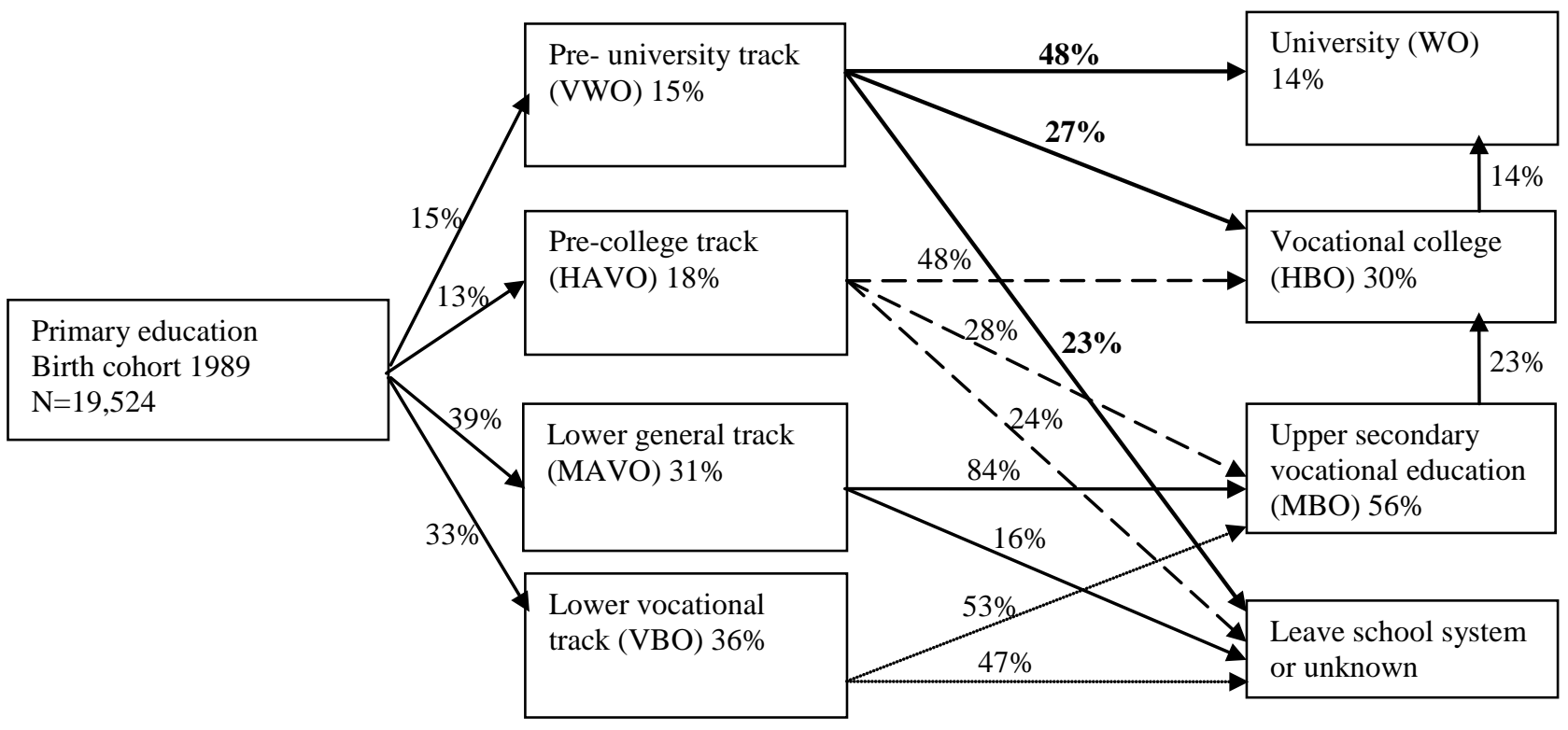

Source: CBS cohort 1989; own calculations.

Note: The arrows represent the transition from one track to another track and add up to $100 \%$. The figures in the boxes represent the highest completed level of secondary or postsecondary education. These can differ from the inflow as a result of up- or downward mobility. For the transition at age 12 we have information for all students. For the transition at age 17-18 we lack information for students who dropped out of the panel or who left school altogether; they are taken together in the category "Leave school system or unknown." 
Figure A2.4: Transition at age 12, from primary education to preuniversity track (VWO). Log odds ratios representing the expected size of parental education inequalities if only primary or secondary effects are operating (relative to 'low education')

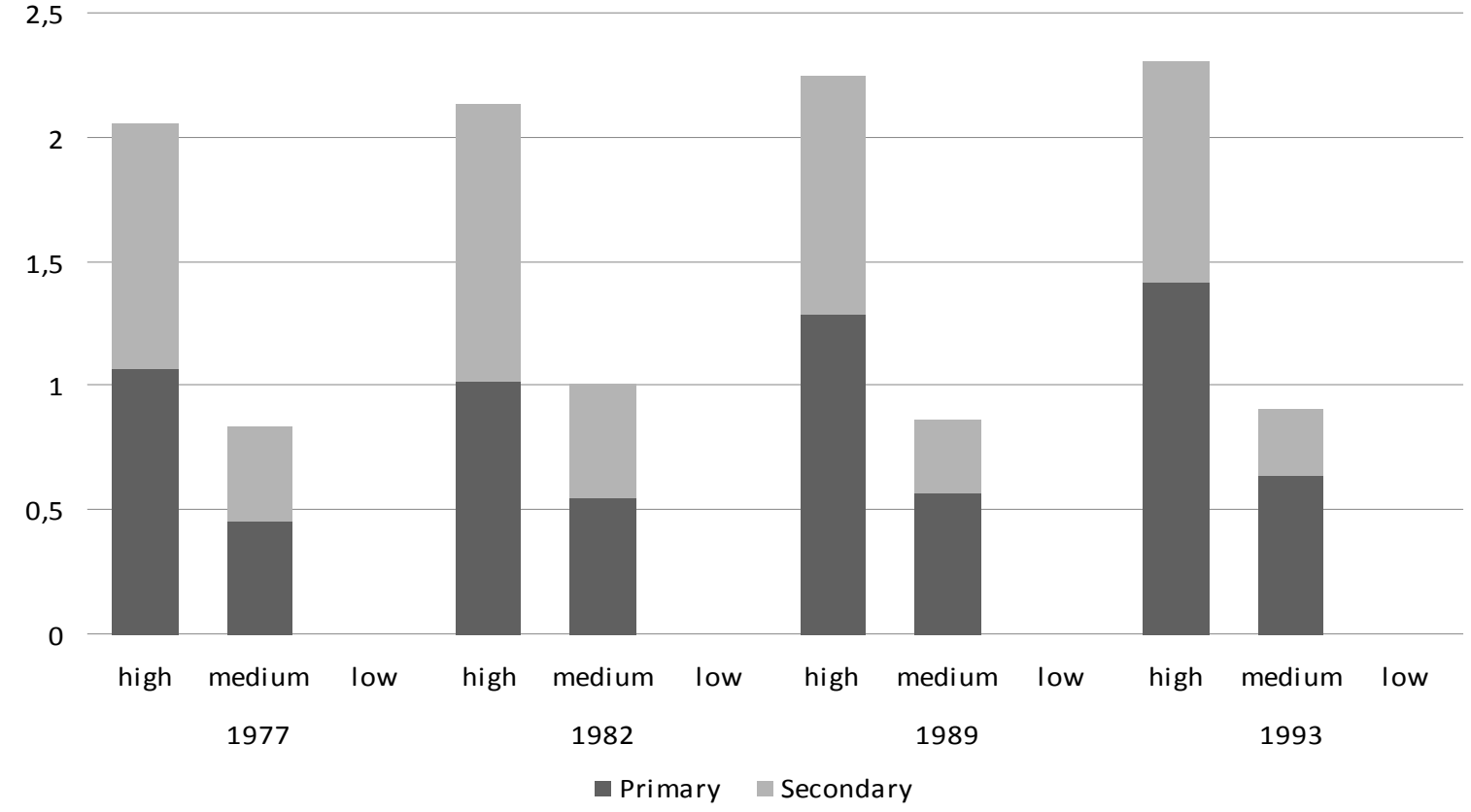

Source: CBS cohorts 1977, 1982, 1989, 1993; our calculations

Figure A2.5: Transition at age 12, from primary education to precollege track (HAVO) or preuniversity track (VWO). Log odds ratios representing the expected size of parental education inequalities if only primary or secondary effects are operating (relative to 'low education')

$$
2,5
$$

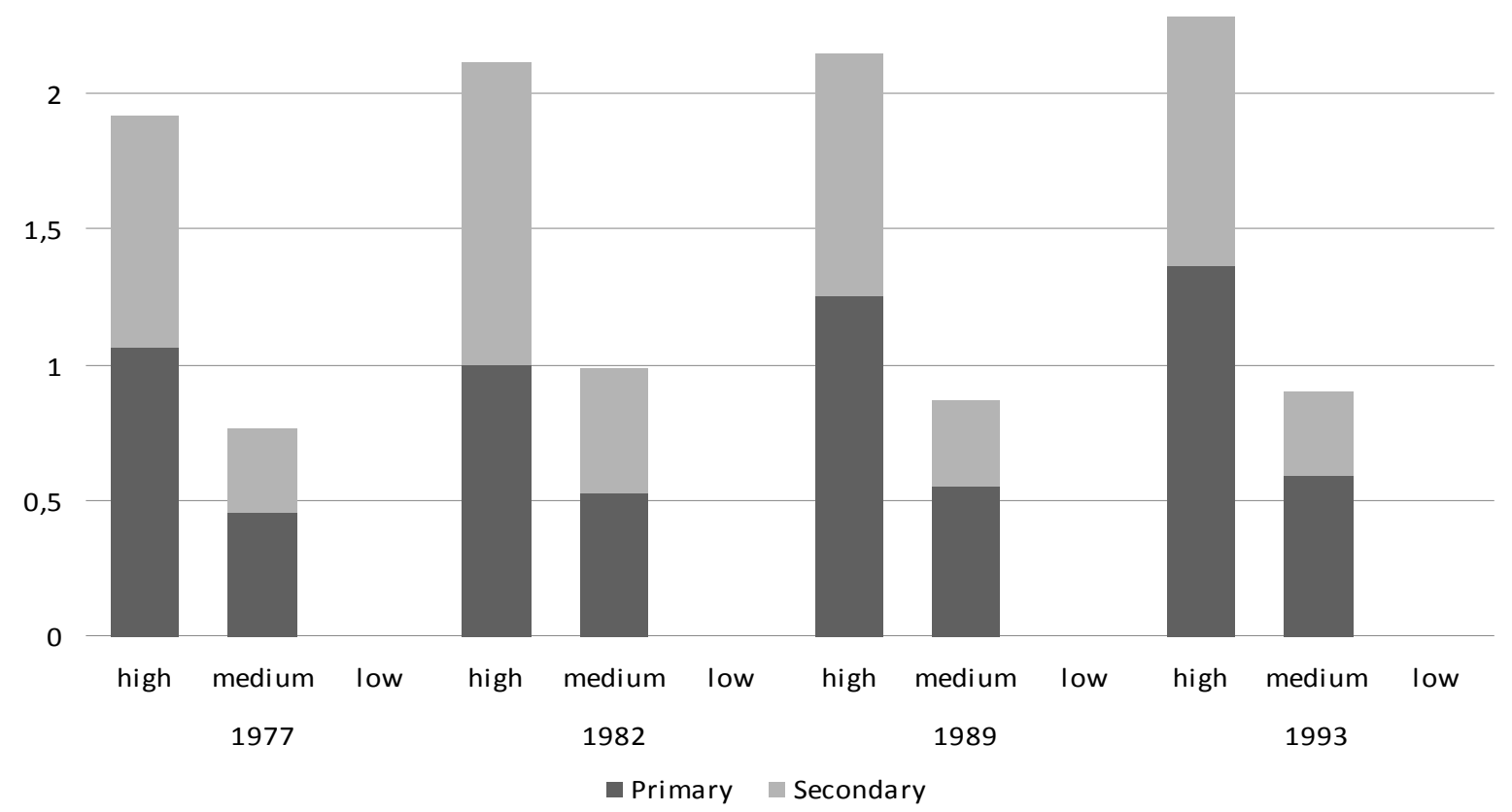

Source: CBS cohorts 1977, 1982, 1989, 1993; our calculations 
Figure A2.6: Transition at age 17 or 18, from preuniversity track (VWO) to university (WO). Log odds ratios representing the expected size of parental education inequalities if only

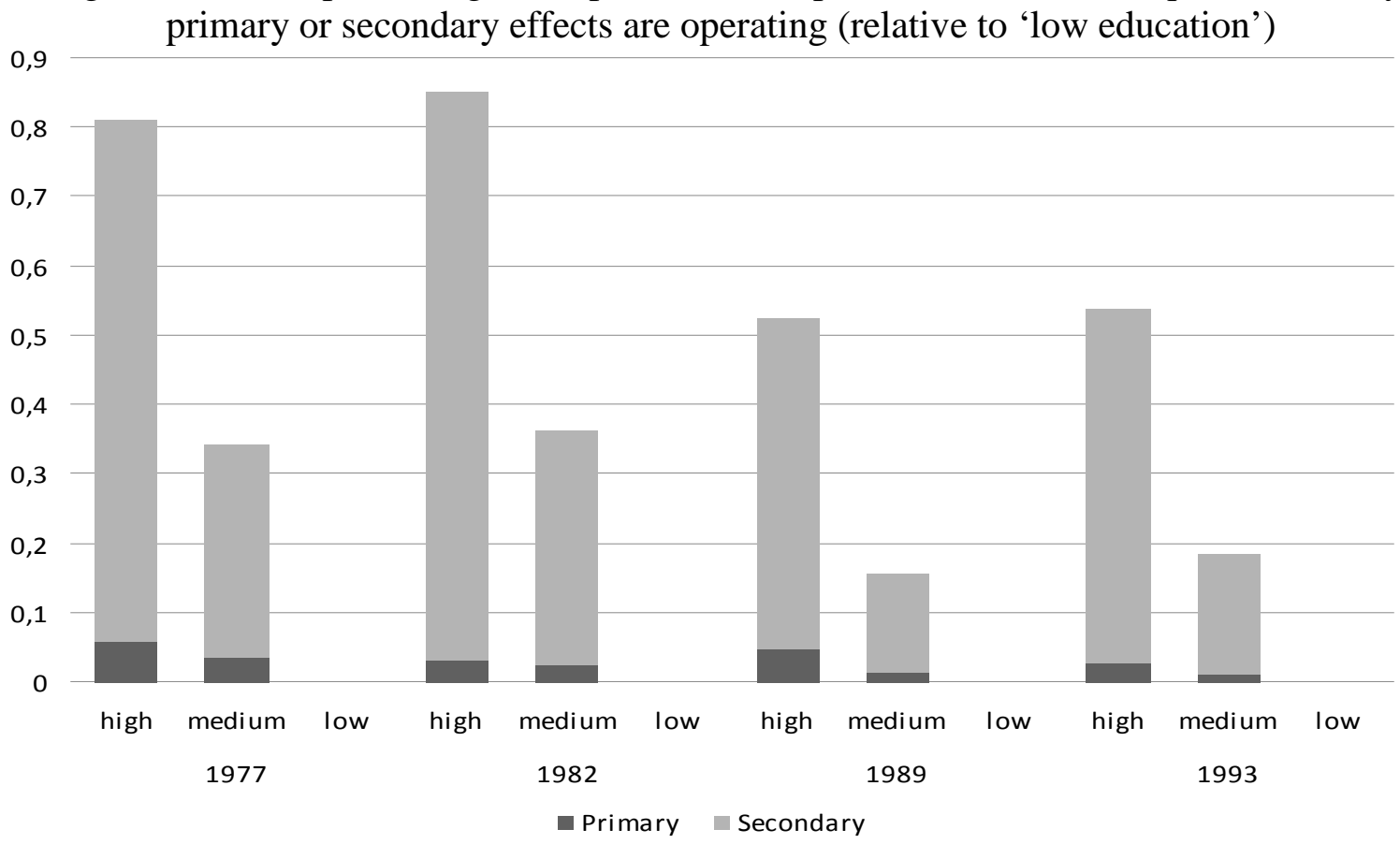

Source: CBS cohorts 1977, 1982, 1989, 1993; our calculations.

Figure A2.7: Transition at age 17 or 18, from precollege (HAVO)/preuniversity track (VWO) to vocational college (HBO) or university (WO). Log odds ratios representing the expected size of parental education inequalities if only primary or secondary effects are operating (relative to 'low education')

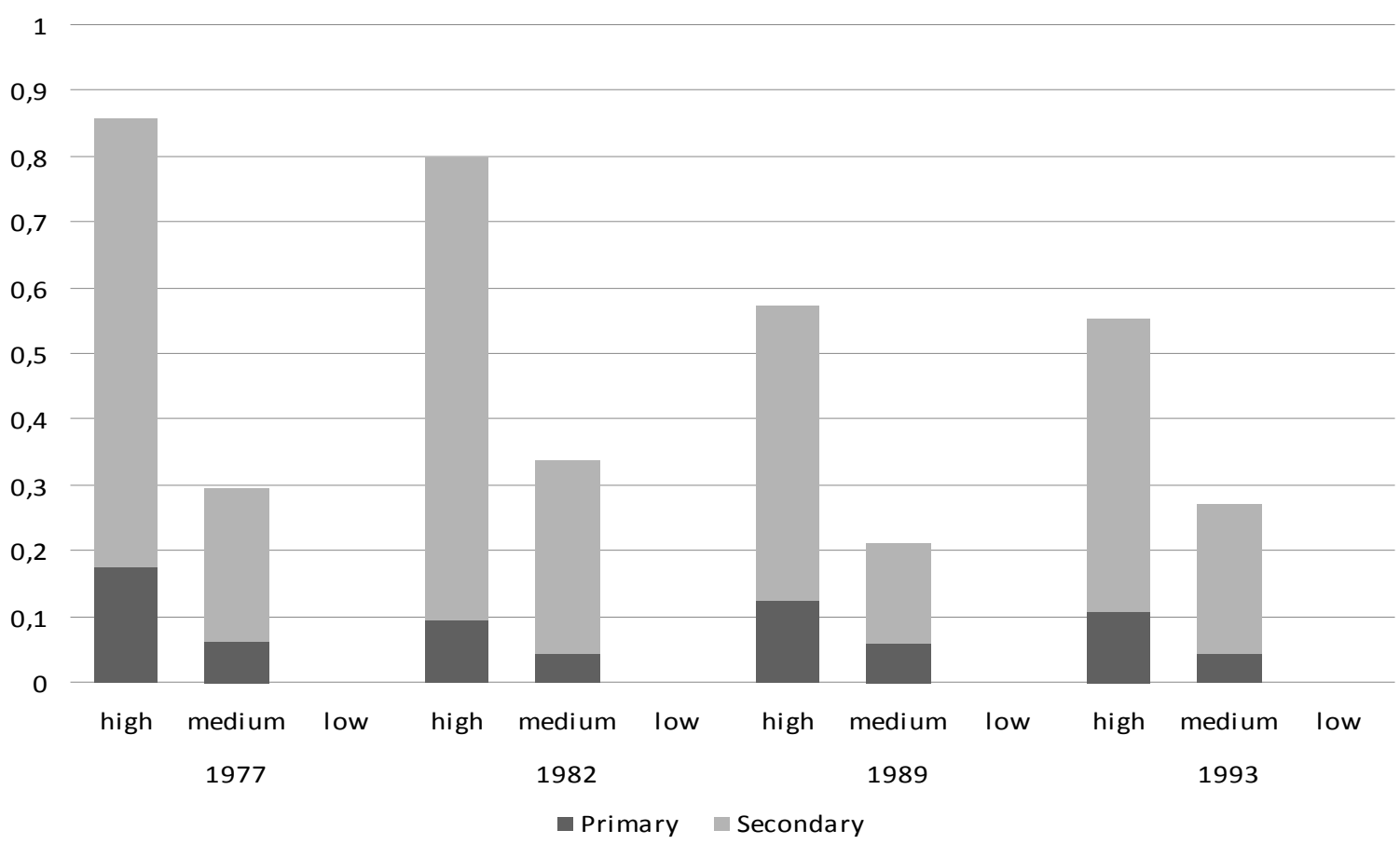

Source: CBS cohorts 1977, 1982, 1989, 1993; our calculations. 
Table A2.1: Children entering secondary education at age 12 by parental education, school cohorts 1977, 1982, 1989, 1993 (\%)

\begin{tabular}{lrrrrr}
\hline & \multicolumn{5}{c}{ Child enters } \\
& vbo & mavo & havo & vwo & \\
\hline Education parents 1977 & & & & & \\
$\quad$ Low & 39 & 42 & 10 & 9 & 100 \\
$\quad$ Medium & 21 & 46 & 15 & 18 & 100 \\
$\quad$ High & 7 & 32 & 19 & 42 & 100 \\
Education parents 1982 & & & & & \\
$\quad$ Low & 47 & 37 & 9 & 7 & 100 \\
$\quad$ Medium & 22 & 43 & 17 & 18 & 100 \\
$\quad$ High & 8 & 30 & 22 & 40 & 100 \\
Education parents 1989 & & & & & \\
$\quad$ Low & 48 & 38 & 8 & 6 & 100 \\
$\quad$ Medium & 28 & 44 & 15 & 13 & 100 \\
$\quad$ High & 8 & 33 & 21 & 38 & 100 \\
Education parents 1993 & & & & & \\
$\quad$ Low & 55 & 30 & 9 & 6 & 100 \\
$\quad$ Medium & 32 & 38 & 16 & 14 & 100 \\
$\quad$ High & 10 & 26 & 24 & 40 & 100 \\
\hline
\end{tabular}

Source: CBS cohorts 1977, 1982, 1989, 1993; our calculations.

Table A2.2: Means and standard deviation of standardized performance scores at age 15, 1989 and 1993 school cohorts

\begin{tabular}{lrlrl}
\hline & \multicolumn{2}{c}{1989} & \multicolumn{1}{c}{1993} \\
\hline Parents' education & & & & \\
Low & -0.34 & $(0.922)$ & -0.36 & $(0.910)$ \\
Medium & 0.08 & $(0.923)$ & 0.05 & $(0.937)$ \\
High & 0.63 & $(0.995)$ & 0.54 & $(0.946)$ \\
\hline
\end{tabular}

Source: CBS cohorts 1989, 1993; our calculations. 
Appendix

Table A2.3: Estimated factual and counterfactual transition rates (\%), from primary education to precollege track (HAVO) or pre-university track (VWO), age 12

\begin{tabular}{|c|c|c|c|c|c|c|c|c|c|c|c|c|c|}
\hline & & \multicolumn{3}{|c|}{1977} & \multicolumn{3}{|c|}{1982} & \multicolumn{3}{|c|}{1989} & \multicolumn{3}{|c|}{1993} \\
\hline & & \multicolumn{3}{|c|}{ Choice } & \multicolumn{3}{|c|}{ Choice } & \multicolumn{3}{|c|}{ Choice } & \multicolumn{3}{|c|}{ Choice } \\
\hline & & Low & Medium & High & Low & Medium & High & Low & Medium & High & Low & Medium & High \\
\hline \multirow[t]{3}{*}{ Performance } & Low & 19 & 24 & 35 & 17 & 24 & 38 & 14 & 18 & 28 & 15 & 20 & 31 \\
\hline & Medium & 27 & 33 & 46 & 25 & 35 & 51 & 22 & 28 & 41 & 24 & 30 & 44 \\
\hline & High & 40 & 48 & 61 & 35 & 46 & 62 & 36 & 44 & 58 & 41 & 49 & 64 \\
\hline
\end{tabular}

Source: CBS cohorts 1977, 1982, 1989, 1993; our calculations.

Table A2.4: Estimated factual and counterfactual transition rates (\%), from precollege track (HAVO) or preuniversity track (VWO) to vocational college (HBO) or university (WO), conditional transition at age 17 or 18

\begin{tabular}{|c|c|c|c|c|c|c|c|c|c|c|c|c|c|}
\hline & \multicolumn{3}{|c|}{1977} & \multicolumn{3}{|c|}{1982} & \multicolumn{3}{|c|}{1989} & \multicolumn{3}{|c|}{1993} \\
\hline & & \multicolumn{3}{|c|}{ Choice } & \multicolumn{3}{|c|}{ Choice } & \multicolumn{3}{|c|}{ Choice } & \multicolumn{3}{|c|}{ Choice } \\
\hline & & Low & Medium & High & Low & Medium & High & Low & Medium & High & Low & Medium & High \\
\hline \multirow[t]{3}{*}{ Performance } & Low & 41 & 47 & 58 & 47 & 54 & 64 & 53 & 57 & 64 & 53 & 58 & 63 \\
\hline & Medium & 43 & 48 & 60 & 48 & 56 & 65 & 55 & 59 & 66 & 54 & 59 & 64 \\
\hline & High & 45 & 51 & 62 & 50 & 57 & 67 & 56 & 60 & 67 & 55 & 61 & 66 \\
\hline
\end{tabular}

Source: CBS cohorts 1977, 1982, 1989, 1993; our calculations. 
Appendix

Table A2.5: Estimated factual and counterfactual transition rates (\%) of all students to vocational college (HBO) or university (WO), unconditional transition at age 17 or 18

\begin{tabular}{|c|c|c|c|c|c|c|c|c|c|c|c|c|c|}
\hline & & \multicolumn{3}{|c|}{1977} & \multicolumn{3}{|c|}{1982} & \multicolumn{3}{|c|}{1989} & \multicolumn{3}{|c|}{1993} \\
\hline & & \multicolumn{3}{|c|}{ Choice } & \multicolumn{3}{|c|}{ Choice } & \multicolumn{3}{|c|}{ Choice } & \multicolumn{3}{|c|}{ Choice } \\
\hline & & Low & Medium & High & Low & Medium & High & Low & Medium & High & Low & Medium & High \\
\hline \multirow[t]{3}{*}{ Performance } & Low & 12 & 17 & 27 & 10 & 16 & 27 & 10 & 13 & 22 & 9 & 14 & 21 \\
\hline & Medium & 16 & 22 & 34 & 14 & 23 & 36 & 14 & 19 & 30 & 14 & 20 & 30 \\
\hline & High & 23 & 31 & 46 & 20 & 31 & 46 & 23 & 29 & 43 & 23 & 32 & 45 \\
\hline
\end{tabular}

Source: CBS cohorts 1977, 1982, 1989, 1993; our calculations.

Table A2.6: Relative importance of secondary effects. Unconditional transition at age 17 or 18 to vocational college (HBO) or university (WO), using performance scores at age 15,

1989 and 1993 school cohorts

\begin{tabular}{llc}
\hline & 1989 & 1993 \\
\hline Medium /Low & 0.26 & 0.36 \\
High /Low & 0.39 & 0.49 \\
High /Medium & 0.48 & 0.57 \\
Average & 0.38 & 0.47 \\
\hline
\end{tabular}


Figure A3.1: Academic performance and transition probabilities to academic tracks in secondary education, school cohort 1977

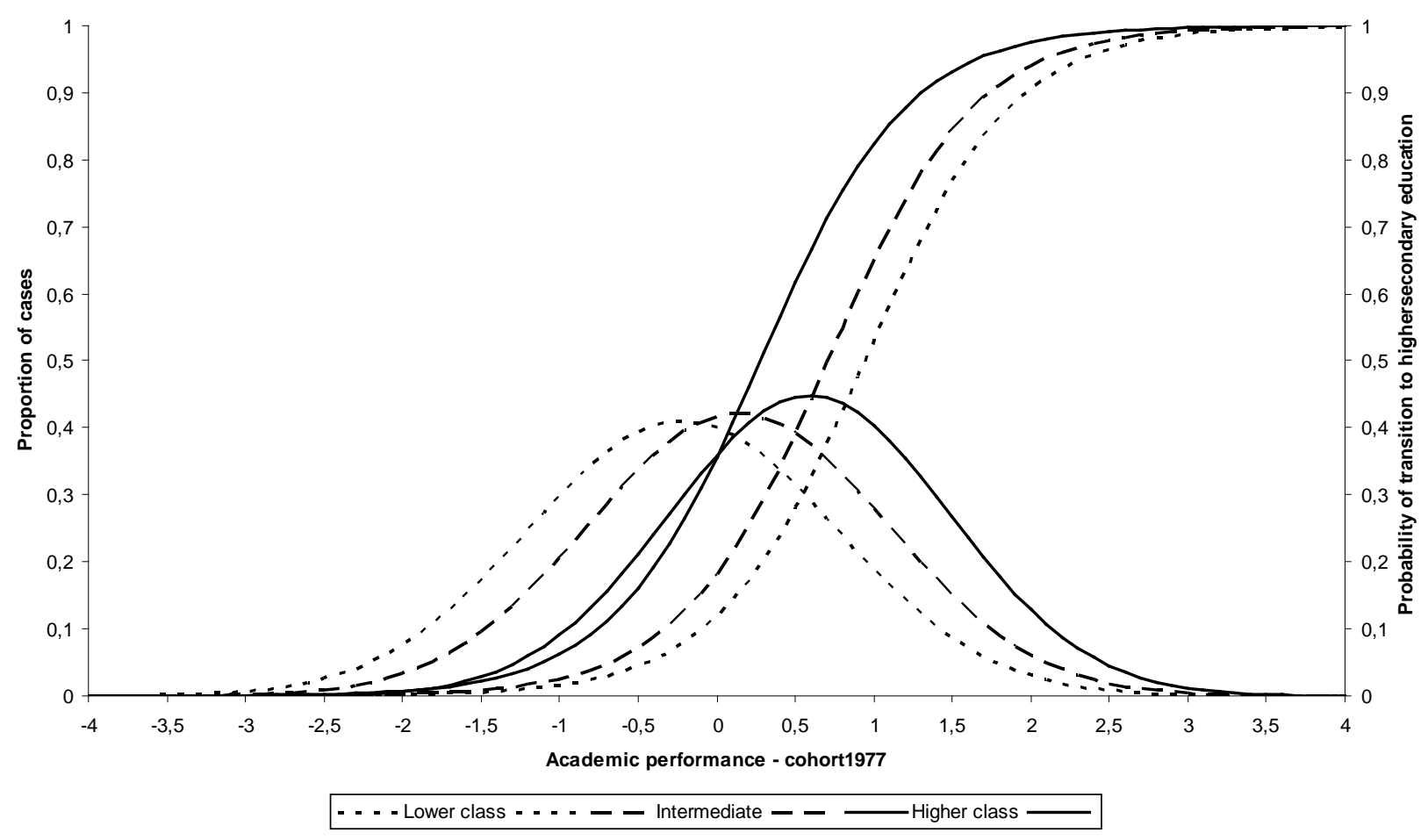

Figure A3.2: Academic performance and transition to academic tracks in postsecondary education, school cohort 1977

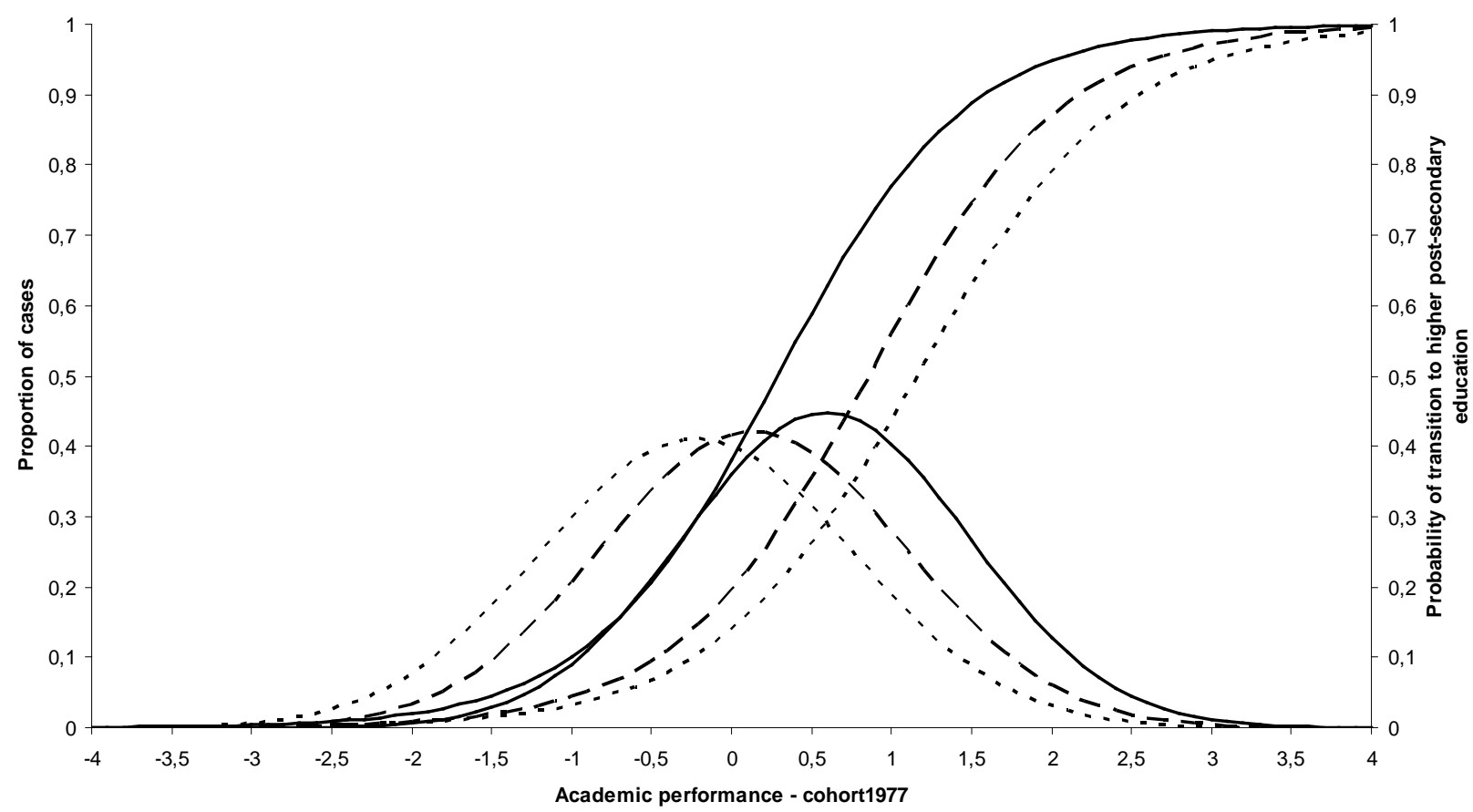

- . - - Lower class - . - - - Intermediate $-\div-$ Higher class - 
Figure A3.3: Academic performance and transition probabilities to academic tracks in secondary education, school cohort 1989

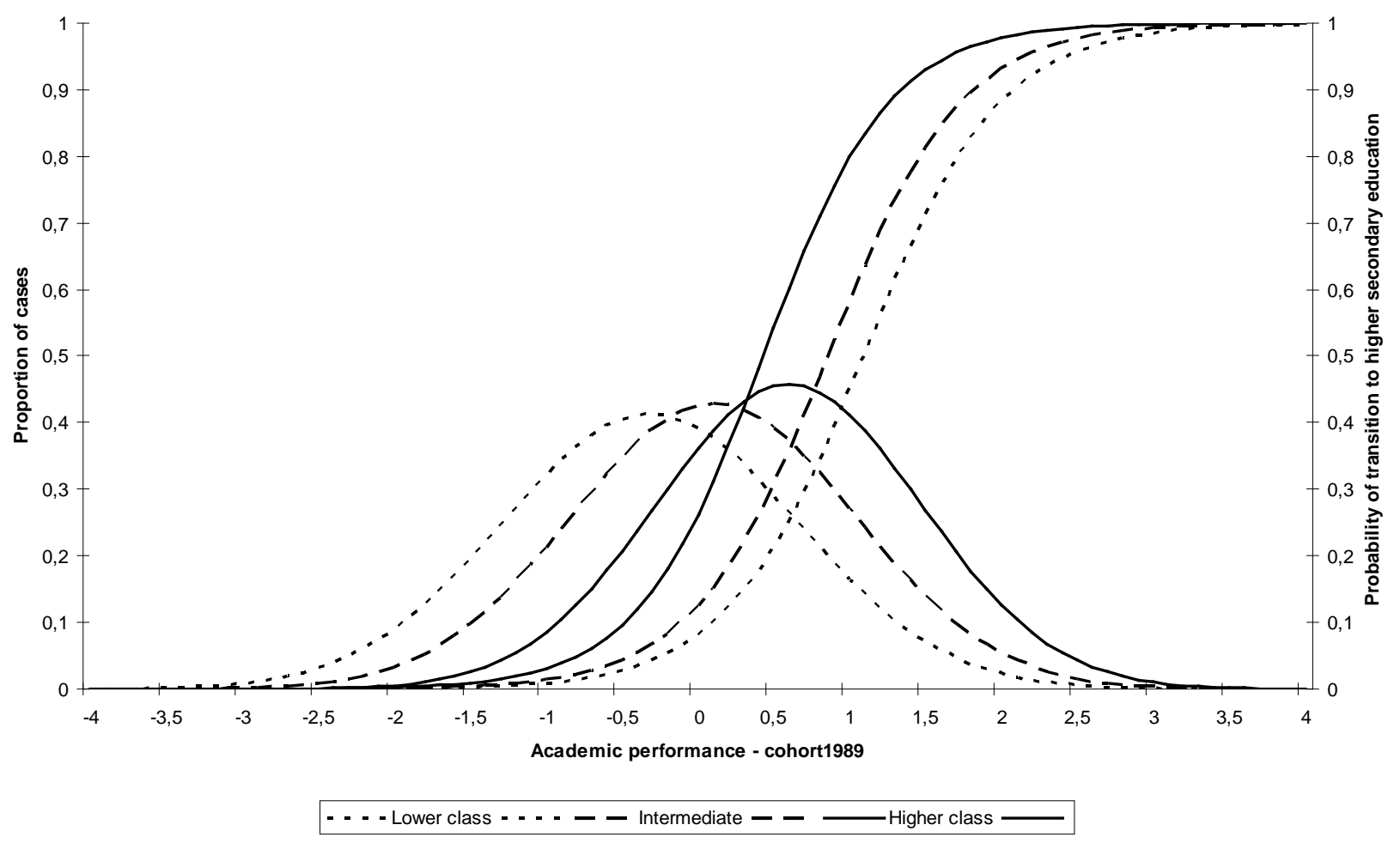

Figure A3.4: Academic performance and transition to academic tracks in postsecondary education, school cohort 1989

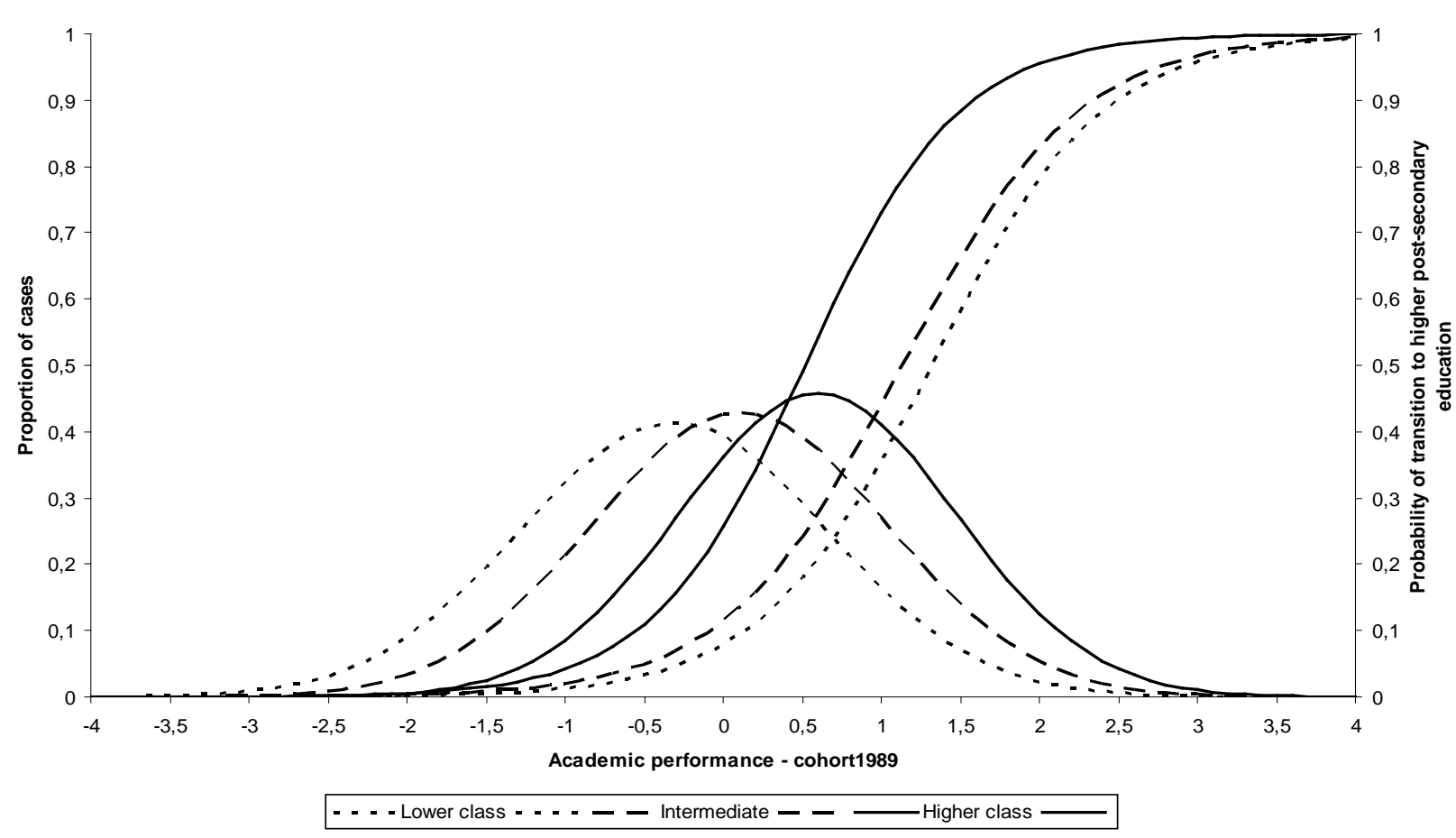


Appendix

Table A4.1: Multinomial logit regression on males’ study field choice (predicted marginal effects)

\begin{tabular}{|c|c|c|c|c|c|c|}
\hline All males & Agriculture & Engineering & Economics & Health & Other & $\begin{array}{l}\text { General } \\
\text { secondary }\end{array}$ \\
\hline Math score & $\begin{array}{l}-0.004 \\
(0.004)\end{array}$ & $\begin{array}{l}-0.026^{* * *} \\
(0.008)\end{array}$ & $\begin{array}{l}0.030 * * * \\
(0.006)\end{array}$ & $\begin{array}{l}0.002 \\
(0.002)\end{array}$ & $\begin{array}{l}-0.008^{* *} \\
(0.004)\end{array}$ & $\begin{array}{l}0.007 \\
(0.007)\end{array}$ \\
\hline Language score & $\begin{array}{l}-0.012^{* * *} \\
(0.003)\end{array}$ & $\begin{array}{l}-0.061^{* * *} \\
(0.007)\end{array}$ & $\begin{array}{l}0.030 * * * \\
(0.006)\end{array}$ & $\begin{array}{l}0.001 \\
(0.002)\end{array}$ & $\begin{array}{l}0.012^{* *} \\
(0.004)\end{array}$ & $\begin{array}{l}0.030 * * * \\
(0.007)\end{array}$ \\
\hline IQ score & $\begin{array}{l}-0.003 \\
(0.003)\end{array}$ & $\begin{array}{l}0.029 * * * \\
(0.007)\end{array}$ & $\begin{array}{l}-0.016^{* *} \\
(0.005)\end{array}$ & $\begin{array}{l}-0.001 \\
(0.002)\end{array}$ & $\begin{array}{l}0.001 \\
(0.003)\end{array}$ & $\begin{array}{l}-0.010 * \\
(0.006)\end{array}$ \\
\hline $\mathrm{N}$ & 6422 & & & & & \\
\hline Pseudo R2 & 0.014 & & & & & \\
\hline $\begin{array}{l}\text { Lower educated } \\
\text { males }\end{array}$ & Agriculture & Engineering & Economics & Health & Other & $\begin{array}{l}\text { General } \\
\text { secondary }\end{array}$ \\
\hline Math score & $\begin{array}{l}-0.013^{* *} \\
(0.004)\end{array}$ & $\begin{array}{l}-0.068 * * * \\
(0.011)\end{array}$ & $\begin{array}{l}-0.007 \\
(0.005)\end{array}$ & $\begin{array}{l}-0.001 \\
(0.001)\end{array}$ & $\begin{array}{l}-0.009 * \\
(0.006)\end{array}$ & $\begin{array}{l}0.098 * * * \\
(0.011)\end{array}$ \\
\hline Language score & $\begin{array}{l}-0.009 * \\
(0.005)\end{array}$ & $\begin{array}{l}-0.085^{* * *} \\
(0.011)\end{array}$ & $\begin{array}{l}-0.011^{* *} \\
(0.005)\end{array}$ & $\begin{array}{l}0.002 \\
(0.001)\end{array}$ & $\begin{array}{l}0.001 \\
(0.006)\end{array}$ & $\begin{array}{l}0.102^{* * *} \\
(0.011)\end{array}$ \\
\hline IQ score & $\begin{array}{l}-0.003 \\
(0.004)\end{array}$ & $\begin{array}{l}0.013 \\
(0.010)\end{array}$ & $\begin{array}{l}0.001 \\
(0.004)\end{array}$ & $\begin{array}{l}-0.002 * \\
(0.001)\end{array}$ & $\begin{array}{l}0.000 \\
(0.005)\end{array}$ & $\begin{array}{l}-0.009 \\
(0.010)\end{array}$ \\
\hline $\mathrm{N}$ & 3196 & & & & & \\
\hline Pseudo R2 & 0.039 & & & & & \\
\hline $\begin{array}{l}\text { Medium educated } \\
\text { males }\end{array}$ & Agriculture & Engineering & Economics & Health & Other & $\begin{array}{l}\text { General } \\
\text { secondary }\end{array}$ \\
\hline Math score & $\begin{array}{l}-0.019 * * \\
(0.007)\end{array}$ & $\begin{array}{l}-0.042 * * \\
(0.014)\end{array}$ & $\begin{array}{l}-0.046^{* *} \\
(0.014)\end{array}$ & $\begin{array}{l}-0.009 * * \\
(0.004)\end{array}$ & - & $\begin{array}{l}0.115 * * * \\
(0.016)\end{array}$ \\
\hline Language score & $\begin{array}{l}-0.033^{* * *} \\
(0.006)\end{array}$ & $\begin{array}{l}-0.073^{* * *} \\
(0.013)\end{array}$ & $\begin{array}{l}-0.016 \\
(0.013)\end{array}$ & $\begin{array}{l}-0.011^{* *} \\
(0.005)\end{array}$ & - & $\begin{array}{l}0.133^{* * *} \\
(0.014)\end{array}$ \\
\hline IQ score & $\begin{array}{l}-0.006 \\
(0.006)\end{array}$ & $\begin{array}{l}0.022 * \\
(0.012)\end{array}$ & $\begin{array}{l}0.051 * * * \\
(0.012)\end{array}$ & $\begin{array}{l}0.002 \\
(0.005)\end{array}$ & - & $\begin{array}{l}0.034 * * \\
(0.012)\end{array}$ \\
\hline $\mathrm{N}$ & 1856 & & & & & \\
\hline Pseudo R2 & 0.067 & & & & & \\
\hline
\end{tabular}


Table A4.2: Multinomial logit regression on females’ study field choice (predicted marginal effects)

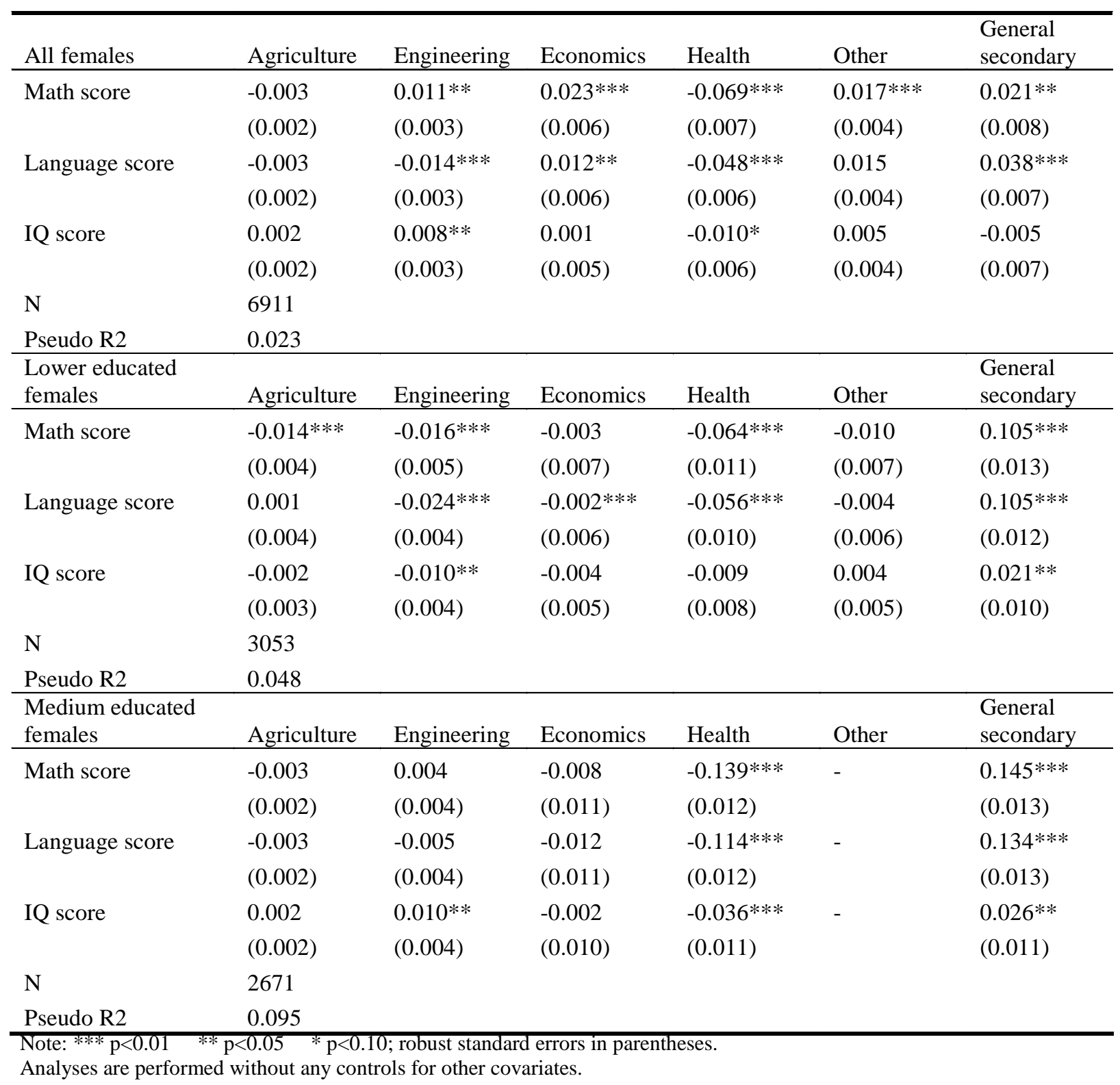


Table A5.1: Descriptive statistics on father's and son's earnings

\begin{tabular}{|c|c|c|c|c|c|c|c|c|c|}
\hline & Mean & SD & Min & Max & $\begin{array}{l}\text { 1st } \\
\text { pctile }\end{array}$ & $\begin{array}{l}\text { 25th } \\
\text { pctile }\end{array}$ & median & $\begin{array}{l}\text { 75th } \\
\text { pctile }\end{array}$ & $\begin{array}{l}\text { 99th } \\
\text { pctile }\end{array}$ \\
\hline Father's earnings in 1995 (Euro) & 43181 & 16355 & 13899 & 114886 & 16955 & 32248 & 39346 & 49587 & 101994 \\
\hline Log father's earnings in 1995 (Euro) & 10.610 & 0.349 & 9.540 & 11.652 & 9.738 & 10.381 & 10.580 & 10.811 & 11.533 \\
\hline Son's earnings in 2008 (Euro) & 47087 & 14729 & 11221 & 101706 & 16905 & 37156 & 44967 & 54951 & 90366 \\
\hline Log son's earnings in 2008 (Euro) & 10.710 & 0.322 & 9.326 & 11.530 & 9.735 & 10.523 & 10.714 & 10.914 & 11.412 \\
\hline
\end{tabular}

Note: Father's earnings are calculated in 2008 earnings, deflated by consumer price index. Earnings of fathers and sons are truncated at the top and bottom 1 percent.

Table A5.2: Descriptive statistics for the full and selected sample

\begin{tabular}{|c|c|c|c|c|c|c|}
\hline & \multicolumn{2}{|c|}{ Full sample } & \multicolumn{4}{|c|}{ Selected sample } \\
\hline & Mean & SD & Mean & SD & Min & Max \\
\hline \multicolumn{7}{|l|}{ Surveyed via son } \\
\hline \multicolumn{7}{|l|}{ Cognitive skills at age 12} \\
\hline Math score & 0.195 & $(1.06)$ & 0.251 & $(1.04)$ & -2.03 & 2.07 \\
\hline Language score & -0.150 & $(1.07)$ & -0.085 & $(1.03)$ & -2.96 & 2.20 \\
\hline IQ test score & -0.175 & $(1.05)$ & -0.126 & $(1.04)$ & -3.79 & 3.41 \\
\hline Years of schooling & 13.26 & (3.35) & 13.65 & (3.28) & 6 & 19 \\
\hline Did not repeat classes in primary school & 0.92 & $(0.28)$ & 0.93 & $(0.25)$ & 0 & 1 \\
\hline General studies & 0.14 & $(0.34)$ & 0.09 & $(0.29)$ & 0 & 1 \\
\hline Engineering studies & 0.46 & $(0.50)$ & 0.49 & $(0.50)$ & 0 & 1 \\
\hline Economics studies & 0.23 & $(0.42)$ & 0.23 & $(0.42)$ & 0 & 1 \\
\hline Agriculture and other studies & 0.06 & $(0.24)$ & 0.05 & $(0.22)$ & 0 & 1 \\
\hline Social sciences and law studies & 0.05 & $(0.23)$ & 0.06 & $(0.25)$ & 0 & 1 \\
\hline \multicolumn{7}{|l|}{ Surveyed via parents } \\
\hline Number of books read by parents & 1.54 & $(1.96)$ & 1.60 & $(1.81)$ & 0 & 44 \\
\hline $\begin{array}{l}\text { Parents visit concert/museum/theatre regularly } \\
\text { Social capital at age } 15\end{array}$ & 0.55 & $(0.50)$ & 0.57 & $(0.49)$ & 0 & 1 \\
\hline $\begin{array}{l}\text { Social status of the neighborhood } \\
\text { Proxy for son's permanent income }\end{array}$ & -0.057 & $(0.94)$ & 0.029 & $(0.83)$ & -3.751 & 3.354 \\
\hline Log house property value son & 12.25 & $(0.34)$ & 12.23 & $(0.31)$ & 9.95 & 14.45 \\
\hline
\end{tabular}

Note: the selected sample considers father-son pairs with valid earnings information for both generations. 
Table A5.3: Pairwise correlations of selected variables

\begin{tabular}{|c|c|c|c|c|c|c|c|c|c|c|c|}
\hline & $\begin{array}{l}\text { Log } \\
\text { earnings } \\
\text { father }\end{array}$ & $\begin{array}{l}\text { Log } \\
\text { earnings } \\
\text { son } \\
\end{array}$ & $\begin{array}{l}\text { Math } \\
\text { skills } \\
\text { son } \\
\end{array}$ & $\begin{array}{l}\text { Languag } \\
\text { e skills } \\
\text { son } \\
\end{array}$ & $\begin{array}{l}\text { IQ test } \\
\text { son }\end{array}$ & $\begin{array}{l}\text { Years of } \\
\text { schooling } \\
\text { son }\end{array}$ & $\begin{array}{l}\text { Son did } \\
\text { not } \\
\text { repeat } \\
\text { classes } \\
\end{array}$ & $\begin{array}{l}\text { Number } \\
\text { of books } \\
\text { parents } \\
\text { read }\end{array}$ & $\begin{array}{l}\text { Parents } \\
\text { visit } \\
\text { concerts/ } \\
\text { museums }\end{array}$ & $\begin{array}{l}\text { Social } \\
\text { status } \\
\text { neigh- } \\
\text { borhood }\end{array}$ & $\begin{array}{l}\text { Log } \\
\text { house } \\
\text { property } \\
\text { value son }\end{array}$ \\
\hline Log earnings son & $0.254 *$ & 1.000 & & & & & & & & & \\
\hline Math skills son & $0.271^{*}$ & $0.315^{*}$ & 1.000 & & & & & & & & \\
\hline Language skills son & $0.267^{*}$ & $0.268 *$ & $0.665 *$ & 1.000 & & & & & & & \\
\hline IQ test son & $0.128 *$ & $0.128 *$ & $0.289 *$ & $0.235 *$ & 1.000 & & & & & & \\
\hline Years of schooling son & $0.316^{*}$ & $0.359 *$ & $0.569 *$ & $0.538 *$ & $0.201^{*}$ & 1.000 & & & & & \\
\hline Son did not repeat classes & $0.077 *$ & $0.129 *$ & $0.224 *$ & $0.184 *$ & $0.076^{*}$ & $0.216^{*}$ & 1.000 & & & & \\
\hline $\begin{array}{l}\text { Number of books parents } \\
\text { read } \\
\text { Parents visit }\end{array}$ & $0.160 *$ & $0.054 *$ & $0.101 *$ & $0.108^{*}$ & $0.082 *$ & $0.131 *$ & 0.029 & 1.000 & & & \\
\hline concerts/museums & $0.205^{*}$ & $0.067 *$ & $0.172 *$ & $0.168 *$ & 0.022 & $0.213^{*}$ & $0.055^{*}$ & $0.183 *$ & 1.000 & & \\
\hline Social status neighborhood & $0.273^{*}$ & $0.167 *$ & $0.213 *$ & $0.196 *$ & $0.076^{*}$ & $0.175 *$ & $0.062 *$ & $0.073 *$ & $0.120 *$ & 1.000 & \\
\hline $\begin{array}{l}\text { Log house property value } \\
\text { son }\end{array}$ & $0.104 *$ & $0.296 *$ & $0.134 *$ & $0.096 *$ & $0.042 *$ & $0.149 *$ & $0.105 *$ & -0.030 & -0.009 & $0.151^{*}$ & 1.000 \\
\hline
\end{tabular}

Note: $* p<0.05$ 
Table A5.4: Relationships between transition mechanisms, father's log earnings and son's log earnings (selected sample)

\begin{tabular}{|c|c|c|c|c|c|c|c|c|c|c|c|c|c|c|}
\hline Dependent variable & \begin{tabular}{|l} 
Father's lo \\
{$[0]$} \\
coeff
\end{tabular} & $\begin{array}{l}\text { earnings } \\
\text { SE }\end{array}$ & $\begin{array}{l}\text { [1] } \\
\text { coeff }\end{array}$ & SE & $\begin{array}{l}{[2]} \\
\text { coeff }\end{array}$ & SE & $\begin{array}{l}{[3]} \\
\text { coeff }\end{array}$ & $\begin{array}{l}\text { Son's lo } \\
\text { SE }\end{array}$ & $\begin{array}{l}\text { g earnings } \\
\text { [4] } \\
\text { coeff }\end{array}$ & SE & $\begin{array}{l}{[5]} \\
\text { coeff }\end{array}$ & SE & $\begin{array}{l}{[6]} \\
\text { coeff }\end{array}$ & SE \\
\hline Math performance & $0.709^{* * *}$ & $(0.071)$ & $0.063^{* * *}$ & $(0.009)$ & & & $0.023 * *$ & $(0.010)$ & & & & & $0.021 * *$ & $(0.010)$ \\
\hline Language performance & $0.813^{* * *}$ & $(0.073)$ & $0.037 * * *$ & $(0.009)$ & & & 0.014 & $(0.009)$ & & & & & 0.010 & $(0.009)$ \\
\hline IQ test & $0.309 * * *$ & $(0.080)$ & $0.016^{* *}$ & $(0.007)$ & & & $0.012^{*}$ & $(0.006)$ & & & & & $0.012^{*}$ & $(0.006)$ \\
\hline $\begin{array}{l}\text { Years of schooling } \\
\text { Field of study (Ref. health) }\end{array}$ & $2.286^{* * *}$ & $(0.186)$ & 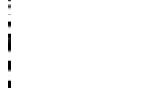 & & $0.047^{* * *}$ & $(0.003)$ & $0.038 * * *$ & $(0.003)$ & & & & & $0.038 * * *$ & $(0.003)$ \\
\hline $\begin{array}{l}\text { General } \\
\text { Engineering }\end{array}$ & $\begin{array}{l}0.039 \\
-0.269 * * *\end{array}$ & $\begin{array}{l}(0.024) \\
(0.041)\end{array}$ & 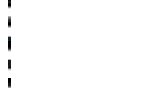 & & $\begin{array}{l}0.164^{* * *} \\
0.108^{* * *}\end{array}$ & $\begin{array}{l}(0.039) \\
(0.032)\end{array}$ & $\begin{array}{l}0.135^{* * *} \\
0.099^{* *}\end{array}$ & $\begin{array}{l}(0.039) \\
(0.031)\end{array}$ & & & & & $\begin{array}{l}0.134^{* * *} \\
0.098^{* *}\end{array}$ & $\begin{array}{l}(0.038) \\
(0.031)\end{array}$ \\
\hline Economics & $0.088 * *$ & $(0.032)$ & $\vdots$ & & $0.111^{* * *}$ & $(0.032)$ & $0.107 * * *$ & $(0.033)$ & & & & & $0.106 * * *$ & $(0.033)$ \\
\hline Agriculture and other & 0.022 & $(0.017)$ & 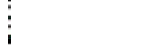 & & -0.018 & $(0.046)$ & -0.023 & $(0.045)$ & & & & & -0.024 & $(0.045)$ \\
\hline Social science and law & $0.077^{* * *}$ & $(0.015)$ & 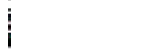 & & 0.050 & $(0.042)$ & 0.047 & $(0.042)$ & & & & & 0.043 & $(0.042)$ \\
\hline $\begin{array}{l}\text { Son did not repeat classes in } \\
\text { primary school }\end{array}$ & $\begin{array}{l}0.026 \\
\vdots\end{array}$ & $(0.019)$ & i & & $0.095^{* * *}$ & $(0.027)$ & $0.084^{* * *}$ & $(0.026)$ & & & & & $0.084 * * *$ & $(0.026)$ \\
\hline $\begin{array}{l}\text { Number of books parents read } \\
\text { Parents visit concert/museum }\end{array}$ & $0.810^{* * *}$ & $(0.122)$ & i & & & & & & 0.004 & $(0.004)$ & & & -0.002 & $(0.003)$ \\
\hline regularly & $0.270^{* * *}$ & $(0.036)$ & ! & & & & & & $0.054 * * *$ & $(0.016)$ & & & 0.000 & $(0.015)$ \\
\hline SES score neighborhood & $0.548^{* * *}$ & $(0.063)$ & & & & & & & & & $0.061^{* * *}$ & $(0.009)$ & $0.033^{* * *}$ & $(0.008)$ \\
\hline R-squared & & & 0.126 & & 0.200 & & 0.214 & & 0.010 & & 0.034 & & 0.224 & \\
\hline $\mathrm{N}$ & & & 1386 & & 1386 & & 1386 & & 1386 & & 1386 & & 1386 & \\
\hline
\end{tabular}

Note: $* p<0.1 ; * * p<0.05 ; * * * p<0.01$. Robust standard errors are in parentheses. The sample is selected to sons who have valid information on earnings and house property value.

Earnings of fathers and sons are truncated at the bottom and top 1 percent. All coefficients are reported as marginal effects. Column [0] shows the results from regressions of each variable on father's earnings, conditional on father's age and age squared. Columns [1]-[6] show sequential earnings equations; e.g. the effect of cognitive skills, schooling etc. on son’s earnings. 
Table A5.5: Accounting for the relationship between father's log earnings and son's log earnings

\begin{tabular}{|c|c|c|c|c|c|c|}
\hline & [1] & [2] & [3] & [4] & [5] & [6] \\
\hline Math performance & 0.0447 & & 0.0163 & & & 0.0149 \\
\hline Language performance & 0.0301 & & 0.0114 & & & 0.0081 \\
\hline IQ test & 0.0049 & & 0.0037 & & & 0.0037 \\
\hline Sum of cognitive skills & 0.0797 & & 0.0314 & & & 0.0267 \\
\hline Years of schooling & & 0.1074 & 0.0869 & & & 0.0869 \\
\hline \multicolumn{7}{|l|}{ Field of study (Ref. health) } \\
\hline General & & 0.0064 & 0.0053 & & & 0.0052 \\
\hline Engineering & & -0.0291 & -0.0266 & & & -0.0264 \\
\hline Economics & & 0.0098 & 0.0094 & & & 0.0093 \\
\hline Agriculture and other & & -0.0004 & -0.0005 & & & -0.0005 \\
\hline Social science and law & & 0.0039 & 0.0036 & & & 0.0033 \\
\hline Son did not repeat classes in primary school & & 0.0025 & 0.0022 & & & 0.0022 \\
\hline Sum of schooling & & 0.1005 & 0.0803 & & & 0.0800 \\
\hline Number of books parents read & & & & 0.0032 & & -0.0016 \\
\hline Parents visit concert/museum regularly & & & & 0.0146 & & 0.0000 \\
\hline Sum of cultural capital & & & & 0.0178 & & -0.0016 \\
\hline $\begin{array}{l}\text { SES score neighborhood } \\
\text { Sum of social capital }\end{array}$ & & & & & $\begin{array}{l}0.0334 \\
\mathbf{0 . 0 3 3 4}\end{array}$ & $\begin{array}{l}0.0181 \\
\mathbf{0 . 0 1 8 1}\end{array}$ \\
\hline Explained & $0.0797(33 \%)$ & $0.1005(42 \%)$ & $0.1117(47 \%)$ & $\mathbf{0 . 0 1 7 8}(7 \%)$ & $0.0334(14 \%)$ & $0.1232(52 \%)$ \\
\hline Unexplained & 0.1593 & 0.1385 & 0.1273 & 0.2212 & 0.2056 & 0.1158 \\
\hline Total & 0.2390 & 0.2390 & 0.2390 & 0.2390 & 0.2390 & 0.2390 \\
\hline
\end{tabular}




\section{Bibliography}

Allen, J., and R. van der Velden (2012): "Skills for the 21st century: Implications for education,” ROA Research Memorandum 2012/11, Maastricht University.

Almlund, M., A. L. Duckworth, J. J. Heckman, and T. Kautz (2011): “Personality psychology and economics,” IZA Discussion Paper 5500.

Altonji, J. G., and R. Blank (1999): "Race and gender in the labor market,” in Handbook of Labor Economics, vol. 3c, ed. by O. Ashenfelter, and D. Card, pp. 3144-3259. Elsevier Science, Amsterdam.

Arcidiacono, P. (2004): “Ability sorting and the returns to college major," Journal of Econometrics, 121, 343-375.

Bakker, B. F. M., J. Dronkers, and H. Schijf (1982): "Veranderingen in individuele schoolloopbanen tussen 1959 en 1977 in de stad Groningen,” Mens en Maatschapij, 57(3), 253-266.

Bakker, B. F. M., and S. P. Schouten (1991): “Trends in onderwijskansen: Een vergelijking van de overgang naar het voortgezet onderwijs van de generaties geboren rond 1953, 1965, en 1971,” Sociale Wetenschappen, 34, 1-22.

Bakker, B. F. M., and P. G. J. Cremers (1994): "Gelijke kansen in het onderwijs? Een vergelijking van vier cohorten leerlingen in hun overgang naar het voortgezet onderwijs,” Tijdschrift voor Onderwijsresearch, 19, 191-203. 
Baltes, P. B., U. M. Staudinger, and U. Lindenberger (1999): „Lifespan psychology: Theory and application to intellectual functioning,” Annual Review of Psychology, 50, 471-507.

Banabou, R. (1996): “Equity and efficiency in human capital investment: The local connection,” Review of Economic Studies, 63(2), 237-264.

Barnett, W. S. (2008): "Preschool education and its lasting effects: Research and policy implications,” Education and the Public Interest Center and Education Policy Research Unit, Boulder, CO. Download: http://nepc.colorado.edu/files/PB-Barnett-EARLYED_FINAL.pdf

Becker, G. (1964): Human Capital: A Theoretical and Empirical Analysis, with Special Reference to Education. University of Chicago Press, Chicago.

_ (1974): “A theory of marriage,” part II. Journal of Political Economy, 82, 11-26.

Becker, G., and N. Tomes (1979): “An equilibrium theory of the distribution of income and intergenerational mobility,” The Journal of Political Economy, 87(6), 1153-1189.

Björklund, A., and M. Jäntti (1997): “Intergenerational income mobility in Sweden compared to the United States,” The American Economic Review, 87(5), 1009-1018.

Blanchflower, D. G., and A. J. Oswald. (1998): "What makes an entrepreneur?,” Journal of Labor Economics, 16(1), 26-60.

Blanden, J., P. Gregg, and L. Macmillan (2007): “Accounting for intergenerational income persistence: noncognitive skills, ability and education,” The Economic Journal, 117, C43C60.

Blanden, J. (2009). "How much can we learn from international comparisons of intergenerational mobility?,” CEE Discussion Paper 111.

Blau, P. M., and O. D. Duncan (1967): The American Occupational Structure. New York: Wiley.

Blossfeld, H.-P., and Y. Shavit (1993): "Persisting barriers: Changes in educational opportunities in thirteen countries," in Persistent Inequality: Changing Educational 
Attainment in Thirteen Countries, ed. by Y. Shavit, and H.-P. Blossfeld, pp. 1-24. Westview, Boulder, CO.

Booth, A. L., and P. Nolen (2009): “Gender differences in risk behaviour: Does nurture matter?,” Discussion Paper of the Centre for Economic Policy Research 7198.

Borghans, L., R. van der Velden, C. Büchner, J. Coenen, and C. Meng (2008): "Het meten van onderwijskwaliteit en de effecten van recente onderwijsverniewingen,” in Commissie Parlementair Onderzoek Onderwijsvernieuwingen. Tijd voor Onderwijs, Deelrapport IV, pp. 3-114. SDU uitgevers, The Hague.

Borghans, L., B. Golsteyn, J. Heckman, and J. Humphries (2011): “Identification problems in personality psychology,” IZA Discussion Paper 5605.

Bosker, R., A. Hofman, and R. van der Velden (1985): Een generatie geselecteerd. Deel 1: De loopbanen. Interimrapport van het SVO-project 1042. RION, Groningen.

Bosma, H., J. Dronkers, and J. Hagenaars (1991): “The consequences of educational reform: Changes in educational attainment processes of pupils at secondary schools," in Comparative Sociology of Family, Health \& Education. A Volume in Memory of Ferran Valls I. Taberner, ed. by M. J. Pelaez, pp. 647-86. Catedra de Historica del Derecho y de las Instituciones, Facultad de Derecho de la Universidad de Malaga, Malaga, Spain.

Bosma, H., and P. G. J. Cremers (1996): "Sociaal-economische verschillen bij de doorstroom naar de universiteit,” Mens en Maatschapij, 71, 142-253.

Bosman, M. H. (1993). Opvoeden in je eentje: een onderzoek naar de betekenis van het moedergezin voor de onderwijskansen van kinderen. Doctoral dissertation. University of Groningen: http://dissertations.ub.rug.nl/faculties/ppsw/1993/m.h.bosman/

Boudon, R. (1974): Education, Opportunity and Social Inequality. John, Wiley \& Sons, New York.

Bound, J., Z. Griliches, and B. Neumark (1986): "Wages, schooling and IQ of brothers and sisters: Do the family factors differ?,” International Economic Review, 27, 77-105. 
Bourdieu, P. (1973): "Cultural reproduction and social reproduction," in Knowledge, Education, and Cultural Change: Papers in the Sociology of Education, ed. by R. Brown, pp. 71-112. Tavistock, London.

Bourdieu, P., and J.-C. Passeron (1977): Reproduction in Education, Society and Culture. Sage, Beverly Hills.

Breen, R., and J. H. Goldthorpe (1997): "Explaining educational differentials: Towards a formal rational action theory,” Rationality and Society, 9, 275-305.

Breen, R., and J. O. Jonsson (2000): “Analyzing educational careers: A multinomial transition model,” American Sociological Review, 65, 754-772.

Brown, C., and M. Corcoran (1997): "Sex-based differences in school content and the malefemale wage gap,” Journal of Labor Economics, 15(3), 431-465.

Browne, M. W., and R. Cudeck (1993): “Alternative Ways of Assessing Model Fit,” in Testing Structural Equation Models, ed. by K. A. Bollen, and J. S. Long, pp. 136-162. Sage, Beverly Hills, CA.

Buchmann, C., T. DiPrete, and A. McDaniel (2008): “Gender inequalities in education," Annual Review of Sociology, 34, 319-337.

Büchner, C., and R. van der Velden (2013): "How Social Background Affects Educational Attainment over Time in the Netherlands," in Determined to Succeed? Performance versus Choice in Educational Attainment, ed. by M. Jackson, pp. 89-115. Stanford University Press, Stanford, CA.

Buis, M.L. (2010a): “Direct and indirect effects in a logit model,” Stata Journal, 10, 11-29.

(2010b): Inequality of Educational Outcome and Inequality of Educational Opportunity in the Netherlands during the 20th Century. $\mathrm{PhD}$ dissertation. Free University, Amsterdam.

Cameron, S. V., and J. J. Heckman (1993): “The Nonequivalence of High School Equivalents,” Journal of Labor Economics, 11, 1-47. 
Bibliography

Carneiro, P., and J. J. Heckman (2003): "Human capital policy,” in Inequality in America: What Role for Human Capital Policies?, ed. by J. J. Heckman, A. B. Krueger, and B. M. Friedman, pp. 77-239. MIT Press, Cambridge, MA.

Cawley, J., K. Coneely, J. Heckman, and E. Vytlacil (1996): "Measuring the effects of cognitive ability,” NBER Working Paper 5645.

Centraal Bureau voor de Statistiek (CBS) (1987): Standaardonderwijsindeling 1978. Den Haag.

(1992): "Schoolloopbaan en herkomst van leerlingen bij het voortgezet onderwijs; vergelijking tussen de cohorten 1977 en 1982,” Unpublished manuscript.

— (1993): Statistisch Jaarboek 1993. SDU, Den Haag.

Centraal Instituut voor Toetsontwickeling (CITO) (2010): “Terugblik en resultaten 2010: Eindtoets basisonderwijs,” Groep 8, versie April 20, 2010. Centraal Instituut voor Toetsontwikkeling, Arnhem.

Chodorow, N. (1989): Feminism and Psychoanalytic Theory. Yale University Press, New Haven, CT.

Cörvers, F. (2000a): “Inkomensmobiliteit tussen generaties: laagste inkomensklassen weinig mobiel,” Index: feiten en cijfers over onze samenleving, 7(7), 30-31.

(2000b): "Inkomensmobiliteit tussen huishoudens met lage inkomens van generatie op generatie,” Armoedemonitor 2000, SCP/CBS, Den Haag, 93-103.

(2004): "Income mobility of successive generations," in: The poor side of the Netherlands: Results from the Dutch 'Poverty Monitor' 1997-2003, SCP/CBS, The Hague, 120-124.

Coleman, J. S., and T. Hoffer (1987): Public and Private High Schools: The Impact of Communities. Basic Books, New York.

Collins, R. (1971): “Functional and conflict theories of educational stratification,” American Sociological Review, 36, 1002-19. 
Corak, M., and A. Heisz (1999): "The intergenerational earnings and income mobility of Canadian men: Evidence from longitudinal income tax data," Journal of Human Resources, 34, 504-33.

Corak, M. (2006): “Do poor children become poor adults? Lessons for public policy from a cross country comparison of generational earnings mobility," Research on Economic Inequality, 13, 143-88.

Corcoran, M., and P. Courant (1989): "Sex-role socialization, screening by sex, and occupational segregation,” Working Paper, University of Michigan, Ann Arbor.

Cunha, F., and J. J. Heckman (2010): “Investing in our young people,” IZA Discussion Paper 5050 .

Cunha, F., J. J. Heckman, and S. M. Schennach (2010): "Estimating the technology of cognitive and noncognitive skill formation,” Econometrica, Econometric Society, 78(3), 883-931, 05.

Currie, J., and D. Thomas (1999): "Early test scores, socioeconomic status and future outcomes,” NBER Working Paper 6943.

De Graaf, P. M., and H. B. G. Ganzeboom (1993): "Family background and educational attainment in the Netherlands for the 1891-1960 birth cohorts,” in Persistent Inequality: Changing Educational Attainment in Thirteen Countries, ed. by Y. Shavit and H.-P. Blossfeld, pp. 75-100. Westview, Boulder, CO.

De Graaf, P. M., and M. H. J. Wolbers (2003): “The effects of social background, sex, and ability on the transition to tertiary education in the Netherlands," The Netherlands Journal of Social Sciences, 39, 172-201.

De Jong, U. (1982): "Inequality in school career: Gender and class, different problems, different solutions," Paper presented at the $10^{\text {th }}$ World Congress on Sociology, Mexico City. 
De Jong, U, J. Dronkers, and W. E. Saris (1982): "Veranderingen in de schoolloopbanen tussen 1965 en 1977: Ontwikkelingen in de Nederlandse samenleving en in haar onderwijs,” Mens en Maatschapij, 57, 26-54.

Dearden, L. S. Machin, and H. Reed (1997): “Intergenerational mobility in Britain,” Economic Journal, 107, 47-64.

Dronkers, J. (1983): "Have inequalities in educational opportunities changed in the Netherlands? A review of empirical evidence,” The Netherlands Journal of Sociology, 19, 133-50.

(1993): "Educational reform in the Netherlands: Did it change the impact of parental occupation and education?,” Sociology of Education, 66, 262-77.

Dronkers, J., and H. Bosma (1990): “The consequences of educational reform for the unequal educational opportunities of children of different social classes in secondary schools,” Zeitschrift für Sozialisationsforschung und Erziehungssoziologie, 10, 268-278.

Eagly, A. H. (1987): Sex Differences in Social Behavior: A Social Role Interpretation. Erlbaum, Hillsdale, NJ.

Eccles, J. (1986): “Gender-roles and women’s achievement,” Educational Researcher, 15(6), 15-19.

Ellison, G., and A. Swanson (2010): "The gender gap in secondary school mathematics at high achievement levels: Evidence from the American mathematics competition,” Journal of Economic Perspectives, 24(2), 109-128.

Erikson, R., J. H. Goldthorpe, and L. Portocarero (1979): "Intergenerational class mobility in three Western European societies: England, France and Sweden,” British Journal of Sociology, 30(4), 415-441.

Erikson, R., and J. O. Jonsson (1996): “The Swedish context: Educational reform and longterm change in educational inequality," in Can Education Be Equalized?, ed. by R. Erikson and J. O. Jonsson, pp. 65-93. Westview Press, Boulder. 
Erikson, R., and J. H. Goldthorpe (2002): "Intergenerational inequality: A sociological perspective,” Journal of Economic Perspectives, 16, 31-44.

Erikson, R., J. H. Goldthorpe, M. Jackson, M. Yaish, and D. Cox (2005): “On class differentials in educational attainment," Proceedings of the National Academy of Sciences, 102, 9730-33.

Erikson, R. (2007): "Social selection in Stockholm schools: Primary and secondary effects on the transition to upper secondary education," in From Origin to Destination. Trends and Mechanisms in School Stratification Research, ed. by S. Scherer, R. Pollack, G. Otte, and M. Gangl, pp. 58-77. Campus, Frankfurt a. M. and New York.

Francis, B. (2006): “Heroes or zeroes? The discursive position of 'underachieving boys' in English neo-liberal education policy,” Journal of Education Policy, 21(2), 187-200.

Gambetta, D. (1987): Were They Pushed or Did They Jump? Cambridge University Press, Cambridge, UK.

Gamoran, A., M. Nystrand, M. Berends, and P. C. LePore (1995): “An organizational analysis of the effects of ability grouping," American Educational Research Journal, 32(4), 687-715.

Georg, W. (2004): “Cultural capital and social inequality in the life course," European Sociological Review, 20(4), 333-344.

Gluckman, P. D., and M. Hanson (2005): The Fetal Matrix: Evolution, Development, and Disease. Cambridge University Press, Cambridge, UK.

Grawe, N.D. (2003): "Life-cycle bias in the estimation bias of intergenerational earnings persistence,” Family and Labour Studies, Analytical Studies Branch Research Paper 207. Statistics Canada, Ottawa.

(2004): "Intergenerational mobility for whom? The experience of high and low earnings sons in international perspective,” in Generational Income Mobility in North America and Europe, ed. by M. Corak, pp. 58-89. Cambridge University Press, Cambridge. 
(2006): "Lifecycle bias in estimates of intergenerational earnings persistence," Labour Economics, 13(5), 551-570.

(2008): “Wealth and economic mobility,” part of the Pew-funded Economic Mobility Project. Download:

http://www.economicmobility.org/assets/pdfs/EMP_LitReview_Wealth.pdf

Grogger, J., and E. Eide (1995): "Changes in college skills and the rise in the college wage premium,” Journal of Human Resources, 30(2), 280-310.

Haider, S. and G. Solon (2006): "Life-cycle variation in the association between current and lifetime earnings,” The American Economic Review, 96, 1308-1320.

Hanushek, E. A., and L. Woessmann (2006): "Does educational tracking affect performance and inequality? Differences-in-differences evidence across countries,” Economic Journal, 116(510), C63-C76.

Heckman, J. J. (2006): “Skill formation and the economics of investing in disadvantaged children,” Science, 312, 1900-1902.

(2007): "The economics, technology and neuroscience of human capability formation,” NBER Working Paper 13195.

Heckman, J. J., J. Stixrud, and S. Urzua (2006): “The effects of cognitive and noncognitive abilities on labor market outcomes and social behavior,” Journal of Labor Economics, 24, 411-482.

Heckman, J. J., R. Pinto, and P. Savelyev (2012): “Understanding the mechanisms through which an influential early childhood program boosted adult outcomes," IZA Discussion Paper 7040.

Heineck, G., and S. Anger (2008): "The returns to cognitive abilities and personality traits in Germany,” Discussion Papers, 836, DIW, Berlin.

Herrnstein, R. J., and C. Murray (1994): The Bell Curve: Intelligence and Class Structure in American Life. The Free Press, New York. 
Horn, W. (1969): Prüfsystem für Schul- und Bildungsberatung PSB. Verlag für Psychologie, Göttingen.

Hout, M., and T. Diprete (2004): “What we have learned: RC28's contributions to knowledge about social stratification,” Working Paper, Draft, University of California, Berkeley.

Hustinx, P. W. J., H. Kuyper, M. P. C. van der Werf, and D. Zijsling (2005): Beschrijving Leerlingsbestanden VOCL '89. Gronings Instituut voor onderzoek van onderwijs, opvoeding en ontwikkeling, Groningen.

Hyde, J. S., E. Fennema, and S. J. Lamon (1990): "Gender differences in mathematic performance: A meta-analysis,” Psychological Bulletin, 107(2), 139-55.

Ioannides, Y. M., and L. Datcher Loury (2004): “Job information networks, neighborhood effects, and inequality,” Journal of Economic Literature, 42(4), 1056-1093.

Jackson, M., R. Erikson, J. H. Goldthorpe, and M. Yaish (2007): "Primary and secondary effects in class differentials in educational attainment: The transition to A-level courses in England and Wales,” Acta Sociologica, 50, 211-229.

Jackson, M. (2013): “Introduction: How is inequality of educational opportunity generated? The case for primary and secondary effects,” in Determined to Succeed? Performance versus Choice in Educational Attainment, ed. by M. Jackson, pp. 1-33. Stanford University Press, Stanford, CA.

Jackson, M, and J. O. Jonsson (2013): "Why does inequality of educational opportunity vary across countries? Primary and secondary effects in comparative context," in Determined to Succeed? Performance versus Choice in Educational Attainment, ed. by M. Jackson, pp. 306-337. Stanford University Press, Stanford, CA.

Jenkins, S. (1987): “Snapshots versus movies: 'Lifecycle biases' and the estimation of intergenerational earnings inheritance,” European Economic Review, 31(5), 1149-1158.

Kartsonaki, C., M. Jackson, and D. R. Cox (2013): "Primary and secondary effects: Some methodological issues," in Determined to Succeed? Performance versus Choice in Educational Attainment, ed. by M. Jackson, pp. 34-55. Stanford University Press, Stanford, CA. 
Kloosterman, R., S. Ruiter, P. M. de Graaf, and G. Kraaykamp (2009): "Parental education, children's performance and the transition to higher secondary education: Trends in primary and secondary effects over five Dutch school cohorts (1965-1999),” British Journal of Sociology, 60, 377-98.

Kloosterman, R. (2010): Social Background and Children's Educational Careers. Studies into the Primary and Secondary Effects of Social Background over Transitions and over Time in the Netherlands. PhD dissertation. Radboud University Nijmegen, Nijmegen.

Korthals, R. (2012): "Selection and tracking in secondary education: A cross country analysis of student performance and educational opportunities,” ROA Research Memorandum, 2012/13, Maastricht University.

Kraaykamp, G., K. Van Eijck, and W. Ultee (2010): "Status, class and culture in the Netherlands,” in Social Status and Cultural Consumption, ed. by T. Wing Chan, pp. 15988. Cambridge University Press, Cambridge.

Lindh, T., and H. Ohlsson (1996): "Self-employment and windfall gains: Evidence from the Swedish lottery,” Economic Journal, 106(439), 1515-1526.

Mare, R. D. (1979): “Social background composition and educational growth,” Demography, $16,55-71$.

(1980): "Social background and school continuation decision," Journal of the American Statistical Association, 75, 295-305.

(1981): “Change and stability in educational stratification,” American Sociological Review, 46, 72-87.

Mazumder, B. (2005): Earnings Mobility in the US: A New Look at Intergenerational Mobility. Unpublished, Federal Reserve Bank of Chicago.

Moonen, L., and M. van den Brakel (2011): Measuring Intergenerational Income Mobility, Centraal Bureau voor de Statistiek. Den Haag/Heerlen, www.cbs.nl. 
Morgan, S. L., M. W. Spiller, and J. J. Todd (2013): “Class origins, high school graduation, and college entry in the United States," in Determined to Succeed? Performance versus Choice in Educational Attainment, ed. by M. Jackson, pp. 279-305. Stanford University Press, Stanford, CA.

Murnane, R. J., J. B. Willet, and F. Levy (1995): “The growing importance of cognitive skills in wage determination,” The Review of Economics and Statistics, 77(2), 251-266.

Murnane, R. J., J. B. Willet, Y. Duhaldeborde, and J. H. Tyler (2000): “How important are the cognitive skills of teenagers in predicting subsequent earnings?,” Journal of Policy Analysis and Management, 19(4), 547-68.

Neal, D. A., and W. R. Johnson (1996): “The role of premarket factors in black-white wage differences,” Journal of Political Economy, 104, 869-895.

Niederle, M., and L. Vesterlund (2010): “Explaining the gender gap in math test scores: The role of competition,” Journal of Economic Perspectives, 24(2), 129-144.

Nybom, M., and J. Stuhler (2011): "Heterogeneous income profiles and life-cycle bias in intergenerational mobility estimation,” IZA Discussion Paper 5697.

Organisation for Economic Cooperation and Development (OECD) (2010): “A family affair: Intergenerational social mobility across OECD countries,” in Going for Growth, ed. by OECD, Chapter 5, pp. 181-198. Paris.

(2011): "Doing better for families, chapter 1: Families are changing”. Download: http://www.oecd.org/els/familiesandchildren/47701118.pdf

Paglin, M., and A. M. Ruffalo (1990): "Heterogeneous human capital, occupational choice, and male-female earnings differences,” Journal of Labor Economics, 8(1, Part 1), 123-44.

Parsons, T. (1970): "Equality and inequality in modern society, or social stratification revisited," in Social Stratification: Research and Theory for the 1970s, ed. by E. O. Lauman, pp. 22-40. Bobbs-Merrill, Indianapolis. 
Peschar, J. L., E. Ten Vergert, and R. Popping (1986): "From father to son and from father to daughter: Educational mobility in Hungary and the Netherlands for the birth cohorts from 1925 to 1955,” Quality and Quantity, 20, 377-403.

Plug, E., Van Praag, B., and J. Hartog (1999): "If we knew ability, how would we tax individuals?,” Journal of Public Economics, 72, 183-211.

Plug, E. (2004): “Estimating the effect of mother's schooling on children's schooling using a sample of adoptees,” The American Economic Review, 94(1), 358-368.

Pong, S. L., and D.-B. Ju (2000): "The effects of change in family structure and income on dropping out of middle and high school,” Journal of Family Issues, 21, 147-169.

Pope, D. G., and J. R. Sydnor (2010): “Geographic variation in the gender differences in test scores,” Journal of Economic Perspectives, 24(2), 95-108.

Raftery, A. E., and M. Hout (1993): "Maximally maintained inequality: Expansion, reform, and opportunity in Irish education, 1921-75,” Sociology of Education, 66, 41-62.

Rauch, J. E. (1993): "Productivity gains from geographic concentration on human capital: Evidence from the cities,” Journal of Urban Economics, 34(3), 380-400.

Rijken, S., I. Maas, and H. B. G. Ganzeboom (2007): “The Netherlands: Access to higher education-institutional arrangements and inequality of opportunity," in Stratification in Higher Education. A Comparative Study, ed. by Y. Shavit, R. Arum, and A. Gamoran, pp. 266-93. Stanford University Press, Stanford, CA.

Schmader, T. (2002): “Gender identification moderates stereotype threat effects on women’s math performance,” Journal of Experimental Social Psychology, 38, 194-201.

Schindler, S. (2010): “Assessing the cumulative impact of primary and secondary effects on the way from elementary to tertiary education: A simulation study for Germany,” Equalsoc Working Paper 2010/2. Download: http://www.equalsoc.org/uploaded_files/publications/sim_schindler_2010.pdf. 
Schindler, S., and D. Reimer (2010): "Primäre und sekundäre Effekte der sozialen Herkunft beim Übergang in die Hochschulbildung,” Kölner Zeitschrift für Soziologie und Sozialpsychologie, 62, 623-653.

Shavit, Y., and H.-P. Blossfeld (1993): Persistent Inequality - Changing Educational Attainment in Thirteen Countries. Westview Press, Boulder.

Sieben, I., J. Huinink, and P. M. De Graaf (2001): "Family background and sibling resemblance in educational attainment: Trends in the former FRG, the former GDR and the Netherlands,” European Sociological Review, 17, 401-30.

Sociaal en Cultureel Planbureau (1998): Van hoog naar laag; van laag naar hoog: De sociaal-ruimtelijke ontwikkeling van wijken tussen 1971 en 1995. Sociaal en Cultureel Planbureau, Den Haag.

Solon, G. (1992): "Intergenerational income mobility in the United States," American Economic Review, 82(3), 393-408.

(2002): “Cross-country differences in intergenerational earnings mobility,” Journal of Economic Perspectives, 16(3), 59-66.

Swift, A. (2005): "Justice, luck and the family: The intergenerational transmission of economic advantage from a normative perspective," in Unequal Chances: Family Background and Economic Success, ed. by S. Bowles, H. Ginits, and M. Osborne-Groves, pp. 256-76. Princeton University Press, Princeton, NJ.

Tieben, N., N. D. De Graaf, and P. M. De Graaf (2010): “Changing effects of family background on transitions to secondary education in the Netherlands. Consequences of educational expansion and reform,” Research in Social Stratification and Mobility, 28, 77-90.

Tieben, N., and M. H. J. Wolbers (2010): “Transitions to post-secondary education in the Netherlands: A trend analysis of unconditional and conditional socio-economic background effects,” Higher Education, 60, 85-100. 
Traag, T., J. van der Valk, R. van der Velden, and R. de Vries (2006): Dertigers op de arbeidsmarkt. ROA-R-2006/2, Maastricht University.

Traag, T., and R.. K. W. van der Velden (2011): "Early school-leaving in the Netherlands. The role of family resources, school composition and background characteristics in early school-leaving in lower secondary education,” Irish Educational Studies, 30, 45-62.

Traag, T. (2012): Early School Leaving in the Netherlands: A Multidisciplinary Study of Risk and Protective Factors Explaining Early School Leaving. PhD-dissertation. Statistics Netherlands, The Hague.

Treiman, D. J. (1970): „Industrialization and social stratification,” in Social Stratification: Research and Theory for the 1970s, ed. by E.O. Lauman, pp. 207-234. Bobbs-Merrill, Indianapolis.

Turner, R. H. (1960): "Sponsored and contest mobility and the school system," American Sociological Review, 25, 855-867.

Van den Brakel, M., and L. Moonen (2009): Is armoede erfelijk? Sociaaleconomische trends, $2 e$ kwartaal 2009. Den Haag/Heerlen: Centraal Bureau voor de Statistiek.

Van der Velden, R. (1991): Sociale Herkomst en Schoolsuccess. Doctoral dissertation, RION, Groningen.

(2011): “De effecten van betrouwbaarheid van onderwijsdiploma’s op arbeidsproductiviteit: Toepassing van een simulatiemodel," in Goede bedoelingen in het onderwijs: Kansen en missers, ed. by J. Dronkers, pp. 27-50. Amsterdam University Press, Amsterdam.

Van Heek, F. (1968): Het Verborgen Talent. Milieu, Schoolkeuze en Schoolgeschicktheid. J. A. Boom, Meppel.

Vrooman, C., and J. Dronkers (1986): “Changing educational attainment processes: Some evidence from the Netherlands,” Sociology of Education, 59, 69-78. 
Bibliography

Weinberger, C. J. (1999): "Mathematical college majors and the gender gap in wages," Industrial Relations, 38, 407-13.

(2001): "Is teaching more girls more math the key to higher wages?,” in Squaring Up: Policy Strategies to Raise Women's Incomes in the United States, ed. by M. C. King, Chapter 11. University of Michigan Press.

Willemse, P. (1987): "Overleving in de bovenstroom van het voortgezet onderwijs: Veranderende milieu-effekten voor jongens en meisjes," Tijdschrift voor Onderwijsresearch, 12, 329-43.

Zax, J., and D. Rees (1998): “Environment, ability, effort and earnings,” Working Paper 9801, Center for Research and Social Policy, Denver.

(2002): “IQ, academic performance, environment, and earnings," The Review of Economics and Statistics, 84(4), 600-616. 


\section{Summary in Dutch}

Onderwijs is een belangrijke factor die bijdraagt aan de persoonlijke en sociale ontwikkeling, en ook positieve effecten heeft op andere dimensies in de levensloop, van een individu (Organisation for Economic Co-operation and Development, 2011). In Nederland, net als in veel andere West-Europese landen, is het bereikte opleidingsniveau een indicatie voor werkgevers om mensen op de arbeidsmarkt te filteren en hun arbeidsproductiviteit te voorspellen. Een hoger opleidingsniveau wordt bijvoorbeeld geassocieerd met hogere arbeidsparticipatie en arbeidsproductiviteit. Echter, de werking van de onderliggende processen van onderwijskeuzes, -afrondingen en arbeidsmarktuitkomsten, is nog niet volledig duidelijk. Er bestaan verschillende mechanismen, zoals sociale achtergrond en cognitieve vaardigheden, die zowel indirect als direct effect hebben op individueel onderwijsen arbeidsmarktsucces. In wisselwerking met de structurele voorwaarden van het onderwijssysteem zijn ze bovendien medeverantwoordelijk voor het niveau van sociale ongelijkheid van onderwijskansen (IEO) in een land (Jackson, 2013). Dit zijn belangrijke uitgangspunten voor sociaal beleid; strategieën die gericht zijn op het verminderen van sociale onderwijsongelijkheid kunnen preciezer worden gedefinieerd, indien deze processen helder zijn en hun verschillende, relatieve impact in deze relatie is geïdentificeerd.

Het doel van deze studie is te onderzoeken in welke mate sociale en cognitieve factoren invloed hebben op het opleidingsniveau en de daaropvolgende arbeidsmarktuitkomsten van jonge mensen in Nederland. Met het oog op sociale ongelijkheid bekijkt dit proefschrift de directe en indirecte effecten van sociale achtergrond op onderwijsresultaten van leerlingen gedurende hun schoolloopbaan voor vier verschillende 
schoolcohorten. Verder bekijkt dit proefschrift in hoeverre de effecten van cognitieve vaardigheden op onderwijs- en arbeidsmarktuitkomsten voor mannen en vrouwen verschillen. Bovendien analyseert dit proefschrift verschillende transmissiemechanismen die de intergenerationele inkomenspersistentie tussen vaders en hun zoons verklaren. Unieke longitudinale onderwijsdata van verschillende Nederlandse schoolcohorten, die gedeeltelijk zijn gecombineerd met informatie over arbeidsmarktuitkomsten, vormen het fundament van de analyses in dit proefschrift. De centrale onderzoeksvragen die we beantwoorden zijn:

- Wat is de relatieve rol van directe en indirecte effecten van sociale achtergrond in het verklaren van onderwijskeuzes en diploma’s, en dus de grootte van ongelijkheid van onderwijskansen (IEO)?

- Wat zijn de onderliggende mechanismen voor het afronden van een bepaald opleidingsniveau en wat is de relatie van deze onderliggende mechanismen met behaalde resultaten op de arbeidsmarkt?

- In welke mate verklaren transmissiemechanismen de relatie tussen het inkomen van vaders en hun zoons?

\section{Directe en indirecte effecten van sociale achtergrond op het opleidingsniveau van} leerlingen over tijd Sociale achtergrond heeft zowel directe effecten op de onderwijskeuzes van een kind, als ook indirect effecten doordat het schoolprestaties beïnvloedt. Boudon (1974) beschreef deze effecten als de primaire (indirecte of prestatiegerelateerde), en secundaire (directe of keuzegerelateerde) effecten van sociale achtergrond. Beiden effecten hebben belangrijke gevolgen voor het niveau van ongelijkheid van onderwijskansen (IEO) in een land. Hoofdstuk 2 onderzoekt op basis van Boudon's theorie het relatieve belang van primaire en secundaire effecten in Nederland bij de overgang naar het hoger secundair en het postsecundair onderwijs voor vier schoolcohorten. ${ }^{1} \mathrm{Om}$ prestatiegerelateerde verschillen van keuzegerelateerde verschillen te kunnen onderscheiden, maken we gebruik van de counterfactual methode, ontwikkeld door Erikson et al. (2005) en Jackson et al. (2007). In een studie over de relatie tussen sociale achtergrond en de overgang naar het hoger secundair onderwijs bij 12-jarigen in Nederland, gebruikten Kloosterman et al. (2009) eerder dezelfde methode. Complementair aan de studie van Kloosterman et al., onderzoekt dit hoofdstuk ook de overgang naar het postsecondair onderwijs bij 17- en 18-

\footnotetext{
${ }^{1}$ Op het hoger secundair onderwijsniveau betreft het de overgang naar hoger algemeen voortgezet onderwijs (HAVO), en voorbereidend wetenschappelijk onderwijs (VWO). Op het postsecundair onderwijsniveau betreft het de overgang naar hoger beroepsonderwijs (HBO), en universiteit (WO).
} 


\section{Summary in Dutch}

jarigen. Voor deze overgang schatten we een conditioneel model dat het behalen van een diploma in het hoger secundair onderwijs als een vereiste stelt. Ook schatten we een onconditioneel model dat alle leerlingen meeneemt die in het postsecundair onderwijs zijn ingestroomd, onafhankelijk van het behalen van een hoger secundair diploma. Bovendien maken we een expliciet onderscheid tussen meer en minder prestigieuze routes binnen het hoger secundair onderwijs (VWO vs. HAVO) en tussen meer en minder prestigieuze routes binnen het postsecundair onderwijs (WO vs. HBO), omdat we ook hier sociale verschillen in onderwijskeuzes veronderstellen.

Het conditionele model laat zien dat over onze onderzoeksperiode van vier cohorten het relatieve belang van secundaire effecten afneemt bij de eerste overgang, en toeneemt bij de tweede overgang. Dit resulteert in vrij stabiele secundaire effecten over tijd. Bovendien worden deze resultaten ondersteund door het feit dat leerlingen uit hoger opgeleide sociale milieus vaker de rechtstreekse route naar de universiteit kiezen. Het onconditionele model toont een matige afname van de relatieve grootte van secundaire effecten. Dit wordt veroorzaakt door het afnemende relatieve belang van deze effecten bij de eerste overgang.

Onze resultaten suggereren dat Nederlandse beleidsacties gericht op het bestrijden van keuzegerelateerde verschillen om ongelijkheid in onderwijskansen te verminderen vooral succesvol zijn geweest bij de overgang van leerlingen naar het hoger secundair onderwijs. Bij de overgang naar het hoger postsecundair onderwijs hebben deze acties weinig tot geen succes geboekt.

Doordat onze resultaten ook aan een cross-nationaal vergelijkend onderzoek bijdragen, kan het relatieve belang van primaire en secundaire effecten in een algemenere context gebracht worden. ${ }^{2}$ Primaire effecten verschillen veel minder in omvang tussen landen en worden minder beïnvloed door de structuur van een specifiek onderwijssysteem dan secundaire effecten (Jackson en Jonsson 2013). De auteurs suggereren dat elk land een basisniveau van IEO kent, afkomstig van sociale achtergrond verschillen in vaardigheden die worden weerspiegeld in schoolprestaties. Boven het basisniveau genereren secundaire effecten de variatie over het totaal niveau van IEO in een land. Ze zijn ermee in grotere mate verantwoordelijk dan primaire effecten voor de variatie in de omvang van IEO, als tussen verschillende landen wordt vergeleken (Jackson en Jonsson, 2013).

\footnotetext{
${ }^{2}$ De landen die zijn meegenomen in deze studie zijn: Denemarken, Duitsland, Engeland, Frankrijk, Italië, Nederland, de Verenigde Staten en Zweden.
} 
Methodologische kwesties met betrekking tot het meten van het effect van sociale achtergrond op opleidingsniveau Gebruikmakend van dezelfde theorie en data als in Hoofdstuk 2 onderzoekt Hoofdstuk 3 de veranderende impact van sociale achtergrond op onderwijskeuzes over tijd. Bovendien voegt dit hoofdstuk een paar belangrijke aspecten toe. Ten eerste testen we met behulp van lineaire regressiemodellen en structural equation models (SEM) de robuustheid van de resultaten van het counterfactual model zoals gepresenteerd in Hoofdstuk 2. Ten tweede, sociale achtergrond beïnvloedt mogelijk niet alleen de overgang van het ene onderwijsniveau naar het andere, maar heeft ook een effect op het bepalen van een diploma (Boudon, 1974). Daarom presenteert Hoofdstuk 3 ook een schatting van primaire en secundaire effecten op de afronding van de verschillende onderwijsniveaus, secundair en postsecundair, gegeven dat het vorige niveau is doorlopen (conditionele model). Ten derde schat Hoofdstuk 3 met behulp van onconditionele modellen het totale effect van sociale achtergrond op het hoogst behaalde onderwijsniveau.

De toepassing van verschillende schattingstechnieken en het gebruik van conditionele en onconditionele modellen geven inzicht in de vraag of het gebruik van verschillende concepten en mechanismen leidt tot het trekken van andere conclusies dan gepresenteerd in Hoofdstuk 2. En of dit bovendien leidt tot andere inzichten voor beleidsvorming. Onze resultaten suggereren dat het gebruik van verschillende schattingsmethoden met betrekking tot de relatieve omvang van de primaire en secundaire effecten inderdaad andere inzichten biedt. De SEM modellen tonen aan dat de lineaire regressiemodellen en de counterfactual modellen secundaire effecten overschatten voor overgangs- en afrondingsfasen in de onderwijsloopbaan. Secundaire effecten bedragen gemiddeld $45 \%$ van het totale effect van sociale achtergrond, bij het gebruik van SEM, en 55\% bij het gebruik van lineaire regressiemodellen en counterfactual modellen. We laten bovendien zien dat de relatieve omvang van secundaire effecten toeneemt op postsecundair onderwijsniveau en dat deze effecten groter zijn bij het afronden van een opleiding dan bij de overgang naar een ander onderwijsniveau. De grootte van ongelijkheid in onderwijskansen die wordt bepaald door de keuzegerelateerde verschillen neemt toe in latere fases van de onderwijsloopbaan. Dit sluit aan bij aannames van theorieën over rationeel handelen en sociale reproductie, maar staat verder af van de moderniseringtheorie. Verder vinden we dat de resultaten die verkregen zijn met behulp van het onconditionele model redelijk in lijn zijn met de resultaten verkregen met behulp van het conditionele model. Dit suggereert dat de paden door het Nederlandse onderwijssysteem vrij rechttoe rechtaan zijn, ondanks het gegeven dat er ook omwegen mogelijk zijn. 


\section{Opleiding, cognitieve vaardigheden en arbeidsmarktsucces van mannen en vrouwen}

Hoofdstuk 4 onderzoekt of, en in welke mate mannen en vrouwen op de arbeidsmarkt worden beloond voor hun cognitieve vaardigheden, afhankelijk van hun bereikte opleidingsniveau en de keuze voor een bepaalde studie. De literatuur suggereert dat individuen over het algemeen op basis van hun formele opleiding in een bepaalde arbeidsmarkt instromen (Heckman et al., 2006). Cognitieve vaardigheden kunnen op hun beurt bijdragen aan extra verschillen in het inkomen uit werk (Paglin en Ruffalo, 1990; Murnane et al., 1995; Arcidiacono, 2004). Volgens human capital theorie worden (investeringen in) scholing en cognitieve vaardigheden beloond - en dit in gelijke mate voor mannen en vrouwen (Becker, 1964). Echter, studies die de samenhang onderzoeken tussen cognitieve vaardigheden, scholing en arbeidsmarktopbrengsten, voor zowel mannen als vrouwen, bieden op dit gebied geen eenduidig inzicht.

In dit hoofdstuk analyseren we mannen en vrouwen van een schoolcohort, voor wie we informatie hebben over cognitieve vaardigheden op 12-jarige leeftijd, sociale achtergrond en opleidingsniveau. Deze informatie is gecombineerd met administratieve gegevens van dezelfde respondenten over hun inkomen, werk- en gezinssituatie, op 35-jarige leeftijd. Voor het meten van cognitieve vaardigheden gebruiken we drie variabelen om mogelijke verschillen in de schattingsresultaten te vergelijken en te evalueren. Twee variabelen bevatten informatie over de uitkomsten van schoolprestatietoetsen op het gebied van wiskunde en taal. Een derde variabele geeft inzicht in de uitkomsten van een non-verbale intelligentietoets.

Voor zowel mannen als vrouwen vinden we een significant effect van cognitieve vaardigheden op inkomen. Vooral wiskundige vaardigheden zijn significant met een stijgend inkomen verbonden. Dit effect is bovendien sterker voor vrouwen dan voor mannen, ook als er voor scholing, sociale achtergrondkenmerken en werksector gecontroleerd is. We schrijven dit resultaat toe aan het feit dat cognitieve vaardigheden een betere voorspeller van scholingsresultaten zijn voor mannen dan voor vrouwen. Voor mannen spelen cognitieve vaardigheden een rol op de arbeidsmarkt, maar slechts indirect, als ze volledig zijn omgezet in opleidingsniveau en specifieke vakgebieden. Ter vergelijking, de selectie van vrouwen in een bepaald onderwijsniveau is minder gebaseerd op hun cognitieve vaardigheden. Hoewel mannen en vrouwen nauwelijks verschillen in hun bereikte opleidingsniveau, suggereren de resultaten dat succes in scholing voor vrouwen meer gerelateerd is aan andere niet waargenomen, non-cognitieve factoren, zoals doorzettingsvermogen en ijver. Desondanks worden de cognitieve vaardigheden van vrouwen volledig op de arbeidsmarkt erkend en boven hun formele opleiding beloond. 


\section{Transmissiemechanismen als verklaring voor intergenerationele inkomenspersistentie}

Hoofdstuk 5 analyseert intergenerationele inkomensmobiliteit tussen vaders en hun zoons in Nederland. Hierbij wordt aangenomen dat het grootste gedeelte van de intergenerationele inkomensmobiliteit wordt overdragen door het effect dat het inkomen van de vader heeft op het opleidingsniveau van de zoon. Dit heeft weer gevolgen voor het eigen inkomen van het kind (Blanden et al., 2007). Zo zijn gezinnen met een hoger inkomen bijvoorbeeld beter in staat om de onderwijskosten van hun kinderen te financieren, wat economische voordelen van generatie op generatie bevordert (Becker en Tomes, 1979; Grawe, 2008). Opleiding, maar ook cognitieve en non-cognitieve vaardigheden, kunnen worden gezien als transmissiemechanismen die worden overdragen van de vorige generatie naar de volgende. We veronderstellen dat deze transmissiemechanismen de intergenerationele inkomenspersistentie verklaren en dus impliciet verantwoordelijk zijn voor de mate van sociale ongelijkheid in een samenleving.

Deze studie beantwoordt de vraag in welke mate transmissiemechanismen de relatie tussen het inkomen van vaders en hun zoons verklaren. We onderzoeken vier verschillende transmissiemechanismen: scholing van de zoon, de cognitieve vaardigheden van de zoon, het cultureel kapitaal van de ouders en het sociaal kapitaal van de woonomgeving van de zoon. ${ }^{3}$ We gebruiken het decompositie model van Blanden et al. (2007) om te onderzoeken in hoeverre deze transmissiemechanismen verantwoordelijk zijn voor intergenerationele inkomenspersistentie in Nederland. Scholing en cognitieve vaardigheden zijn conventionele mechanismen in dit economische model, maar we voegen met het cultureel kapitaal en het sociaal kapitaal twee sociologische constructies bij, die mogelijk nog additionele invloed hebben. Onze resultaten laten zien dat de cognitieve vaardigheden en de scholing van een zoon ongeveer $50 \%$ van de intergenerationele inkomenspersistentie tussen vader en zoon verklaren. Onderwijs verklaart het overgrote deel, terwijl cognitieve vaardigheden vooral een indirect effect hebben via het opleidingsniveau. Deze resultaten zijn in lijn met de resultaten van onderzoek van Blanden et al. (2007) dat voor het Verenigd Koninkrijk werd uitgevoerd. Vooral het vergelijkbaar resultaat voor onderwijs ondersteunt een centraal punt in de sociale ongelijkheid-literatuur, namelijk dat onderwijs sterk medeverantwoordelijk is voor de reproductie van de economische status in het gezin (Blau and Duncan, 1967).

\footnotetext{
${ }^{3}$ Cognitieve vaardigheden van de zoon zijn gemeten aan de hand van schoolprestatietoetsen en een non-verbale intelligentietoets op 12-jarige leeftijd; het cultureel kapitaal van de ouders is gemeten aan de hand van het aantal boeken dat de ouders lazen en hoe vaak ouders musea, concerten en toneelstukken bezochten. Het sociaal kapitaal van de woonomgeving van de zoon is gemeten aan de hand van de sociaal-economische samenstelling van de woonomgeving waar de zoon is opgegroeid.
} 
Het sociaal kapitaal van de woonomgeving en het cultureel kapitaal van de ouders verklaart een extra 6\% van de intergenerationele inkomenspersistentie. Van deze twee mechanismen heeft sociaal kapitaal de hoogst verklarende waarde. Om te testen voor robuustheid, vervangen we het inkomen van de zoon met de waarde van het huis van de zoon, als proxy voor zijn vaste inkomen. De verkregen resultaten zijn nagenoeg hetzelfde als in het oorspronkelijke model. De vier transmissiemechanismen verklaren samen ongeveer $56 \%$ van de intergenerationele inkomenspersistentie tussen vader en zoon. Welke mechanismen de overige $44 \%$ verklaren, zijn in deze studie niet geobserveerd. Het is aannemelijk dat overgedragen specifieke persoonlijkheidskenmerken, zoals risicotolerantie en ondernemersvaardigheden, en spillover effecten van het familievermogen, bijdragen aan intergenerationele inkomenspersistentie.

De conclusie van dit proefschrift is dat verticale sociale verschillen in Nederland - met betrekking tot het opleidingsniveau en de intergenerationele reproductie van economische status - zowel relatief als absoluut in onze onderzoeksperiode waarneembaar blijven. Hoewel onze resultaten laten zien dat het relatieve belang van keuzegerelateerde verschillen binnen het totale IEO is afgenomen over tijd, blijkt het slechts marginaal lager te zijn dan het belang van prestatiegerelateerde verschillen. Zowel primaire als secundaire effecten dragen bij aan het niveau van IEO. Echter, de institutionele kenmerken van het onderwijssysteem zijn medeverantwoordelijk voor de grootte van secundaire effecten, en dat in grotere mate dan voor primaire effecten. Het is dus een belangrijke taak voor het onderwijssysteem om gelijkheid van onderwijskansen te stimuleren, door het introduceren van overheidsinterventies vooral op het gebied van keuzegerelateerde verschillen. Deze keuzegerelateerde verschillen kunnen gemakkelijker worden aangepakt dan prestatiegerelateerde verschillen. Desondanks moeten ook programma's worden bevorderd die sociale verschillen in prestaties verminderen. Om effectief te zijn moeten deze programma's bovendien worden uitgeoefend op voorschoolse leeftijd. Studies over het rendement van dergelijke programma's laten zien dat ze succesvol zijn in het verbeteren van zowel cognitieve als non-cognitieve vaardigheden (bijv. Heckman, 2006.; Heckman et al., 2012). Keuzes voor hogere onderwijsniveaus, echter, zijn ook onderworpen aan economische beperkingen en leiden tot hogere kosten en risico's voor studenten uit kansarme milieus. De toenemende secundaire effecten bij de overgangs- en afrondingsfasen van postsecundair onderwijs in onze analyse wijzen dit uit. Beleid kan ingrijpen met financiële prikkels voor getalenteerde studenten uit lagere sociale milieus, door bijvoorbeeld een lager collegegeld 
aan deze groep leerlingen te vragen. Een andere mogelijkheid is het aanbieden van professionele begeleiding en advies tijdens de schoolloopbaan. Dergelijke acties kunnen nuttig worden aangevuld met herhaalde prestatietoetsen in latere onderwijsstadia, om fouten in onderwijskeuzes te verminderen. Dit is nu nog onderontwikkeld in het Nederlandse onderwijs (Van der Velden, 2011). Herhaalde prestatietoetsen kunnen helpen om zowel de professionele begeleiding als individuele beslissingen gedurende de hele schoolloopbaan continu aan te passen, en mogelijk onderwijsbeslissingen van studenten minder afhankelijk te maken van de familieachtergrond.

Verder laat dit proefschrift significante geslachtsverschillen in taal- en rekenprestaties, maar ook in de keuze voor bepaalde studies zien, bij gelijk talent en opleiding. Met betrekking tot de studiekeuze kan een meer op maat gemaakte, professionele begeleiding voor onderwijsplanning een strategie zijn. Vooral getalenteerde meisjes moeten zo vroeg mogelijk in hun schoolcarrière worden gestimuleerd om hun volledige potentieel te gebruiken, door hen bijvoorbeeld te stimuleren een vak te kiezen waarbij zij hun vaardigheden optimaal kunnen benutten. Echter, integratie van meisjes in door jongens gedomineerde studierichtingen, zoals techniek, kan mislukken als gevolg van geslacht- en leeftijdspecifieke peer-group oriëntaties. Om bètavakken aantrekkelijker te maken voor meisjes en het aandeel meisjes binnen dit type opleiding te verhogen, is het van belang die aspecten van een bepaald bètavak te benadrukken waar meisjes echt in geïnteresseerd zijn. De focus zou minder moeten liggen op vakinhoudelijk aspecten alleen.

Met betrekking tot significante verschillen in taal- en rekenprestaties, dus verschillen in talenten, zijn bovendien alternatieve selectieprogramma's denkbaar die talenten voldoende stimuleren en ontwikkelen. Deze programma's spelen meer in op de keuze voor een bepaalde studie, en zijn gericht op het benutten van specifiek talent in een bepaald vakgebied. De inhoud van deze programma's zou in mindere mate gedreven moeten worden door leeftijd of onderwijsniveau. Dit zou een zeer geïndividualiseerd systeem karakteriseren, en zeker meer financiële middelen eisen voor gekwalificeerd personeel dan het bestaande systeem. 


\section{Biography}

Charlotte Büchner studied sociology at the Free University of Berlin and graduated in 2003. After her studies she worked as a research associate in the German Socio-Economic Panel Study-Department at the German Institute for Economic Research (DIW), and at the Social Science Research Center Berlin (WZB). In 2006, Charlotte started working as a researcher and Ph.D. student at the Research Centre for Education and the Labour Market (ROA) at Maastricht University. Since 2013, Charlotte is employed as a project coordinator at the German Institute for International Pedagogic Research (DIPF) in Berlin. She is affiliated to DIW, ROA, the Network Social Innovation (NSI), and the Network of Excellence on Economic Change, Quality of Life and Social Cohesion (EQUALSOC). 


\section{ROA Dissertation Series}

1. Lex Borghans (1993), Educational Choice and Labour Market Information, Maastricht, Research Centre for Education and the Labour Market.

2. Frank Cörvers (1999), The Impact of Human Capital on International Competitiveness and Trade Performance of Manufacturing Sectors, Maastricht, Research Centre for Education and the Labour Market.

3. Ben Kriechel (2003), Heterogeneity Among Displaced Workers, Maastricht, Research Centre for Education and the Labour Market.

4. Arnaud Dupuy (2004), Assignment and Substitution in the Labour Market, Maastricht, Research Centre for Education and the Labour Market.

5. Wendy Smits (2005), The Quality of Apprenticeship Training, Conflicting Interests of Firms and Apprentices, Maastricht, Research Centre for Education and the Labour Market.

6. Judith Semeijn (2005), Academic Competences and Labour Market Entry: Studies Among Dutch Graduates, Maastricht, Research Centre for Education and the Labour Market.

7. Jasper van Loo (2005), Training, Labor Market Outcomes and Self-Management, Maastricht, Research Centre for Education and the Labour Market.

8. Christoph Meng (2005), Discipline-Specific or Academic? Acquisition, Role and Value of Higher Education Competencies, Maastricht, Research Centre for Education and the Labour Market.

9. Andreas Ammermüller (2007), Institutional Effects in the Production of Education: Evidence from European Schooling Systems, Maastricht, Research Centre for Education and the Labour Market. 
10. Bart Golsteyn (2007), The Ability to Invest in Human Capital, Maastricht, Research Centre for Education and the Labour Market

11. Raymond Montizaan (2010), Pension Rights, human capital development and well-being, Maastricht, Research Centre for Education and the Labour Market.

12. Annemarie Nelen (2012), Part-Time Employment and Human Capital Development, Maastricht, Research Centre for Education and the Labour Market.

13. Jan Sauermann (2013), Human Capital, Incentives, and Performance Out- comes, Maastricht, Research Centre for Education and the Labour Market.

14. Harald Ulrich Pfeifer (2013), Empirical Investigations of Costs and Benefits of Vocational Education and Training, Maastricht, Research Centre for Education and the Labour Market.

15. Charlotte Büchner (2013), Social Background, Educational Attainment and Labor Market Integration: An Exploration of Underlying Processes and Dynamics, Maastricht, Research Centre for Education and the Labour Market. 